\title{
A FINDING AID FOR 25 YEARS OF ADVERTISEMENTS IN THE KODAK ADVERTISING COLLECTION, GEORGE EASTMAN ARCHIVES AND STUDY CENTER
}

\author{
by \\ Sarah Fay, BFA, Ryerson University, 2007.
}

\begin{abstract}
A Thesis Project
Presented to Ryerson University and George Eastman House
\end{abstract}

in partial fulfillment of the requirements for the degree of Masters of Arts in the program of Photographic Preservation and Collections Management

Toronto, Ontario, Canada, 2010

(C) Sarah Fay 2010 



\section{Author's Declaration:}

I hereby declare that I am the sole author of this thesis or dissertation.

I authorize Ryerson University to lend this thesis or dissertation to other institutions or individuals for the purpose of scholarly research.

\section{Sarah Fay}

I further authorize Ryerson University to reproduce this thesis or dissertation by photocopying or by other means, in total or in part, at the request of other institutions or individuals for the purpose of scholarly research.

Sarah Fay 


\begin{abstract}
:
This project is a finding aid for twenty-five years of Kodak Advertisements, which encompasses a written description of the project, an analysis of the results, and the finding aid itself. The finding aid presents an index of each advertisement published between the years of 1900 to 1925 from the Kodak Advertising Collection binders at the George Eastman Study Centre in the George Eastman House Museum of Photography and Film in Rochester, New York. The finding aid pays particular attention to imagebased advertisements, slotting them into one of seven categories: women, children, family, farmers, travel and outdoors, labor, and soldiers.
\end{abstract}




\section{Acknowledgements:}

I would like to thank Kathy Connor, Curator of the George Eastman Legacy Collection for helping me with this project, as well as for her insight into the Kodak Advertising Collection. Additionally, I would like to thank Dan Bish and Jesse Peers, Archivists at the George Eastman Study Centre, for their help and for pulling many, many binders of ads out for me.

My thanks are also heartily extended to my thesis advisor, Elspeth Brown for her support and guidance, as well as her helpful feedback. Thank you also, to my second reader, Bob Burley for giving my thesis its finishing touches. I would also like to thank Alison Nordstrom for her advice and support while working on this thesis.

To my sister Cheryl Fay, my boyfriend Dave Taglialatela, and my roommate and friend D'Arcy White I bestow the deepest thanks for your support and encouragement throughout my education. I truly could not have done this without you.

And finally, I would like to thank my parents, Michelle and Frank Fay who have helped me so much, in grad school and always, and have never hesitated to drive down to Rochester for their daughter. 

I would like to dedicate this thesis to my sister and best friend, Cheryl Fay. 


\section{Table of Contents:}

Introduction 1

An Overview of Kodak and the Kodak Advertising Collection 3

$\begin{array}{lr}\text { Literature Survey } & 12\end{array}$

$\begin{array}{ll}\text { Methodology } & 21\end{array}$

Analysis:

$\begin{array}{ll}\text { Overview } & 25\end{array}$

Advertising and the Media in America During WWI 26

Analysis of Ads $\quad 30$

WWI in Memory $\quad 34$

$\begin{array}{ll}\text { Conclusion } & 39\end{array}$

$\begin{array}{ll}\text { Bibliography } & 41\end{array}$ 


\section{List of Illustrations:}

1. The seven categories in the finding aid 25

2. Snap-shots From Home. 30

3. The Kodak Letter. 25

4. Take a Kodak with you. 44

5. Snap-Shots from Home. 44

6. All out-doors invites your Kodak. 44

7. The Kodak on the Farm. 44

8. Kodak on the Job. 45

9. Get it with a Brownie. 45

10. Keep a Kodak Story of the Children. 45

11. A New Kodak. 45 


\section{List of Appendices:}

Appendix A: Kodak Advertisement Examples

Appendix B: Finding Aid 


\section{Introduction}

This thesis is a practical project- a finding aid for twenty-five years of Kodak Advertisements, which will encompass a written description of the project, an analysis of the results, and the finding aid itself. I have compiled an index of each advertisement published between the years of 1900 to 1925 from the Kodak Advertising Collection binders at the George Eastman Study Centre (GESC) in the George Eastman House Museum of Photography and Film (GEH) in Rochester, New York.

The Library of Congress defines a finding aid as: "A description of an archival collection, usually containing a history of the person or organization that produced the collection and an inventory of its contents. ${ }^{1}$ With this in mind, I will begin the first section by giving an overview of the Kodak Advertising Collection, tracing its mysterious provenance, and describing how it came to be at the GEH. I will discuss relevant sources of information in the literature review and then describe my methodology. The second part of the paper will be the analysis, including a description of inherent categories discovered within the advertisements, and a more detailed study of one of the themes (soldiers).

The Kodak Advertising Collection is a much-used asset at the George Eastman House and it is my goal that the finding aid that I have assembled will assist researchers in the future. At present, the advertisements are only searchable at the binder level. With the addition of this finding aid to the

1. Library of Congress, "Finding Aids and Guides" Rare Book and Special Collections Reading Room, http://www.loc.gov/rr/rarebook/findaid/findaid.html (accessed May 29, 2010). 
study center, the advertisements for this twenty-five year period will be searchable individually. This collection is important to the museum and its history and by assembling this finding aid I have helped to improve access to it. In addition, I hope that my written analysis will enable people to gain a greater understanding of the Kodak material from the beginning of the twentieth century.

This project was completed in partial fulfillment of the requirements for the degree of Masters of Arts in the program of Photographic Preservation and Collections Management. 


\section{An Overview of Kodak and the Kodak Advertising Collection}

George Eastman, founder of the Eastman Kodak company was a self

made man. Unhappy with the large photographic outfits of the time, Eastman set out to create an easier way to take photographs. This led to the invention of the Kodak camera in 1888 . It was lauded as the first camera that could be used by anyone, regardless of his or her skill, age, sex, or economic status. The camera- a box covered in leather- was simple to operate. The camera was aimed at the subject, the button was pressed, and a crank was turned to advance to the next photograph. Kodak cameras came pre-loaded with 100 shots. When the user had taken all the photos, he or she simply shipped the camera back to Eastman Kodak where Kodak employees would process and print the images, re-load the camera, and send everything back to the customer. As Kodak put it early on, it was "Photography with the bother left out."

This was a time of change for advertising. In the late nineteenth century it was seen as a distasteful obligation companies could use to increase knowledge of their brands. These text-based ads gave way to more visually appealing ads featured in magazines. Advertising became something to be taken seriously, by consumers and companies alike, and as a result the quality and the appeal of the ads shot up. Eastman realized this early on, and so began to use advertisements

2. Rochester, NY, George Eastman House George Eastman Legacy Collection, Kodak Advertising Collection, 1895-1907 5 of 8. 
to create "a nation of amateur photographers." ${ }^{3}$

During the years contained in this finding aid, unlike many other corporations, Kodak did not contract their advertising out to an ad agency. Instead, Eastman invented slogans and ad concepts himself. The ads began as simple illustrations of products with information on their availability. As advertising techniques evolved at the end of the 1890's and the early 1900 's, Kodak, led by Eastman and later, advertising director Lewis Bunnell Jones began to use more visually sophisticated means to sell their cameras. Ads no longer featured the camera front and centre. An ever-growing portion of Kodak's monthly ads began to feature people instead. These figures, which were always fashionably dressed, white, middle-class or above men and women, held onto the Kodak as if it was an extension of their fashionable attire. The Kodak became an accessory, part of the lifestyle. The make, model, and technical specifications became less important; what was important was the act of taking photographs. Eastman used his ads to promote photography as a part of life, trying to prevent it as being seen as a passing fad. To do this, he shifted his marketing strategy to include families and children. Ads encouraged parents to photograph children constantly, warning the parents that memories would fade over time, and only a photograph would "keep the story."

3. Elizabeth Brayer, George Eastman : A Biography. (Rochester, NY: University of Rochester Press, 2006), 135. 
Eastman told J. Walter Thompson in the 1880's that "A picture of a pretty girl sells more than a tree or a house..$^{\prime 4}$ and so, he created the most iconic of Kodak ads: The Kodak Girl. Always fashionable, the Kodak Girl appeared in many ads beginning in 1888 and continuing well into the later $20^{\text {th }}$ century. As Nancy West argues, the Kodak Girl's "youthful image would signify the ease, pleasure, and freedom of snapshot photography. ${ }^{\prime 5}$ By using the Kodak Girl, as well as the later introduced Brownie Boy and a host of other men, women and children, Kodak created a marketing campaign that focused not only on how and when to photograph, but highlighted how simple it was to do. Advertisements continued to additionally laude the newest features in the cameras but these advertisements dwindled as time went on, limited to an announcement when a new model was available. The rest of the time, the people in the ads became the demonstrators and promoters of the Kodaks, eliminating the need for camera focused sales advertisements.

What started out as a camera and film company expanded rapidly during the twenty-five year period I surveyed; there are advertisements for a large variety of products. Film and cameras are well represented, but so are chemicals; both for photographic, and general use. The binders from 1920 onwards have more Eastman Organic Chemical ads than they do ads for cameras. In addition to this, there are a multitude of ads for darkroom apparatuses-cutting boards, film tanks, enlargers, rulers, lamps, and much

4. Brayer, 135.

5. Nancy Martha West, Kodak and the Lens of Nostalgia (Charlottesville: University Press of Virginia, 2000), 53. 
more. Many Kodak publications are featured, advertising to the reader how to take better photographs at home, on the farm, and of the children. X-Ray film features prominently in later years, showing images of teeth, gallstones and other internal maladies all diagnosed with Kodak X-Ray film. Of course, each binder also contains many ads featuring happy men, woman and children outdoors lauding the newest Kodak camera-These are the ads which most often are representative of this time period at Kodak, when in reality the ads selling neither camera nor film number just as high.

The Kodak Advertising Collection consists of 708 binders and scrapbooks, put together by the company's ad department over the course of its history. These are made up of several types of binders, as one can assume availability changed over the collection's long history. The binders for the twenty-five year period I surveyed measure 15" wide, 17 1/2" high, and 2 $1 / 2 "$ deep. Each binder contains between 35 and 50 pages that are slipped into plastic protective sheets. The ads themselves are pasted to black or white paper and are sometimes accompanied by penciled-in notes (most often the date). In some cases there is also a date stamped onto the ad, though this is not consistent. These books were compiled by the Eastman Kodak Company and were donated to the George Eastman House in 2003. The collection covers more than 100 years of advertisements- both tear sheets and printed ads - from 1884 to 1988 Before coming to the GEH the ads were stored in Kodak headquarters, located at 343 State St., Rochester 
NY. They were kept in the basement under the jurisdiction of the Kodak library. ${ }^{6}$

As Kodak began to downsize their operations in the late 1990's, librarians were laid off or relocated. The last remaining librarian at Kodak headquarters, Ray Curtin, realized that this material was in danger of being lost therefore in 2001 he invited Kathy Connor, who was curator of the Eastman Legacy Collection at the GEH, to look at the collection in order to see if the GEH would be interested in taking it. Although it does not strictly fall within the collection's mission (to collect material related to the life of George Eastman from the years 1854-1932, with the advertising collection extending beyond this date range), ${ }^{7}$ Kathy agreed to present the material to her acquisition committee. Despite both Kathy and the committee being against the acquisition of the materials, they were brought into the collection in 2003, mostly due to the prompting of the museum's director. If the GEH had not accepted the collection in its entirety, the portion after 1932 would have been sent to the University of Rochester, home to another Kodak archive, but it was thought more appealing to keep the entire collection together. ${ }^{8}$ It is worth noting that the business archive at University of Rochester is extensive and may be useful for researchers interested in the business side of Kodak. It is home to a substantial number of boxes containing business papers from Eastman Kodak, including boxes of advertising manuscripts, seven that I personally scoured in search of in-

6. Kathy Connor, conversation with the author, 20 May 2010.

7. Ibid.

8. Ibid. 
house information on the development of the advertisements. Unfortunately, I was unable to find anything relevant to my thesis but the university's archive would still be a valuable source for other Kodak researchers.

The material was not considered desirable to the GEH committee at the time because not only did it span further than the years of Eastman's life and thus outside the mission of the collection, but also because it came into the collection unorganized and not catalogued. Kodak promised to pay for the shelving of the collection and the moving expenses incurred in the process though, and so Connor planned a movable shelving system for the ads to be housed on. With this plan in place, the advertisements were packed into hundreds of boxes and sent to the GEH in 2003.

The binders that the ads are stored in are perhaps made of materials that are not archival grade. In the 39 binders I surveyed, the ads are pasted to different types of paper slipped into clear binder sheets. The black paper used in some cases is in much better condition than the brittle, white paper used in others. However, black album paper is well known for being acidic and not good for long-term preservation of paper materials. The binders themselves are often overfull, leading to the binder rings opening when being used by a researcher. This poses a higher risk of damage for the ads as, at this point, there is more opportunity to be dropped or mishandled. It is because of these issues that, when the collection was brought into GEH, Kathy Connor had an assessment made for the re-housing of the material. It was estimated that it would cost seventy-five thousand dollars, which is 
obviously much more money than the museum has to spend. ${ }^{9}$ This is partially because of the size of the binders - they are not standard and therefore special housing materials would have to be made for them. With this cost in mind, it is unreasonable to chastise the GEH for how it houses the Kodak Advertising Collection; it was rescued from a basement storage room and moved to a climate-controlled facility, and the binders themselves are adequate protection from the elements. Archivists diligently assist researchers, reducing the improper handling that may occur from an overfull binder. Although there are more effective storage methods, it is a matter of balancing the ideal with the available, and in this case, I believe the collection is being well managed.

When the collection was housed at Kodak, before it arrived at the Eastman House, it was inaccessible to almost everybody. Exceptions were sometimes granted to see the material, such as in the case of Nancy Martin West, author of Kodak and the Lens of Nostalgia, and with Warner Brothers when they asked to access it for material for their parks and films. This has changed drastically with its move to the GEH. The Kodak Advertising Collection can now be seen by anybody who asks, which has both pros and cons. It is positive because this is the purpose of a research collection: to be seen. However, each viewing of the binders places strain on them; brittle paper cracks, the binders break, and there is always a risk to the collection itself. For this reason, other methods of viewing the ads are available. For instance, in many cases a researcher will be content with seeing a facsimile

9. Kathy Connor, conversation with the author, 20 May 2010. 
of the ad, and so binders of photocopies are available at the Study Center for such work. Additionally, the GEH's website has a large number of advertisements available to view online. However, this will not satisfy every researcher and in some cases there is no alternative but to show the original material. When this happens, it is preferable to show the researcher his or her particular request with as little disruption to the rest of the collection as possible. This is where my finding aid will be useful.

Since 2003, there have been several finder's tools applied to the Kodak ads in order to make them more accessible. After the collection's acquisition, Kathy Connor hired archivist Deb Hamil, who spent two years creating index sheets for each binder. These sheets, along with an internal HTML database, give basic information about the content of each binder. Overall themes as well as subjects are listed along with any other information that may be unique to a binder. This information focuses on the ads relating to Kodak cameras, as this is the area that the Study Center receives the most requests. To date, no finding aid has been created focusing on individual advertisements in the binders. This is what my project will change. With a finding aid in place that allows a researcher to search for a specific ad before turning to the binders themselves, the collection will be more accessible than ever before while at the same time it will cut down on unnecessary access to the ads. As it is the ultimate goal of the George Eastman Study Center to facilitate research and discovery, this will allow the GESC to help accommodate research requests on many levels while adhering to the preservation and care of the material required by an institution. Both 
Hamil's finding aid and my own facilitate access to the collection, however Hamil's is only useful at the binder level whereas my own will allow the researcher to view information pertinent to the individual advertisements. 


\section{Literature Survey}

Research for my thesis was concentrated on three distinct areas: the history of advertising, and the United States of America from 1900-1925; the history of Kodak and George Eastman; and research on existing finding aids. Of these three areas, the Kodak ads have the least written on them. While the history of Kodak and George Eastman is widely published, the advertisements themselves are not widely discussed in scholarly papers, likely due to the previously discussed unavailability of the collection itself until recently.

In my study of American history, I found A History of the American People by Paul Johnson to be a comprehensive look at America's entire history. The years 1900-1925 are covered from many angles, including relevant looks at the rise in wealth over the first ten years of the century and the growing gap between the rich and the poor. ${ }^{10}$ Additionally, Johnson looks at the growth of daily newspapers from 1850-1910. There were 260 daily papers in 1870 versus the 2,600 in 1910 - the latter which is the peak number of daily newspapers in American history, meaning more of an audience for Kodak's ads. ${ }^{11}$

Advertising the American Dream- Making Way for Modernity, 19201940 by Roland Marchand was an excellent modern-day look at early twentieth century ads, even though I am interested in a time period earlier than the book highlights. While Marchand mentions Kodak specifically twice

10. Paul Johnson, A History of the American People, (New York: HarperCollins Publishers, 1998), 601.

11. Ibid., 593. 
in his book, both times it is in reference to ads after $1925 .{ }^{12}$ Nevertheless, Marchand's examples of common advertising tactics are quite relevant to the advertisements in the collection, as they share a number of similarities, for example, the chapter "Visual Cliché" where examples of the a familial unit in advertising is illustrated. ${ }^{13}$

Jackson Lears's book Fables of Abundance- A Cultural History of Advertising in America contained interesting ideas about the incorporation of photography into advertising in its chapter "The Persistence and Resurgence of Real Life". Lears mentions the statistic that between 1922 and 1927, advertisements in Ladies Home Journal using photographs increased to twenty-five percent, up from fourteen percent. ${ }^{14}$ This was an interesting statistic, especially considering that Kodak ads featured a higher number of photographic ads much earlier. However, this is hardly surprising considering the nature of Eastman Kodak.

Image Makers by Robert Jackall and Janice M. Hirota examines advertising primarily from the viewpoint of supporting organizations. For example, the chapter on World War I, which I found particularly helpful, wrote about the representation of the war shown to the American people as created by the Committee on Public Information. This committee, founded in 1917 and disbanded in 1919, was created to "sell America's Crusade to the

12. Roland Marchand, Advertising the American Dream: Making Way for Modernity, 1920-1940 (Berkeley: University of California Press, 1985), 72, 134.

13. Ibid., 250.

14. Jackson Lears, Fables of Abundance: A Cultural History of Advertising in America (New York: Basic Books, 1994), 324. 
American Public. ${ }^{\prime 15}$ During the war years, Kodak primarily focused on marketing their products to families of soldiers. Looking at the ads through the ideas of the Committee on Public Information brings new light onto many of the themes inherent in the advertisements themselves.

Photographic Memories by Rob Kroes is an investigation of the photograph, which was helpful in obtaining information on the link between the photograph and memory that I discuss in my analysis of soldier advertisements. ${ }^{16}$ The Past Within Us by Tessa Morris-Suzuki was similarly helpful, as it discusses the ways in which history is disseminated through all forms of media. Though it emphasizes the history of Asia, the themes and ideas translated well to American history. ${ }^{17}$

Both Jay Winter's Remembering War and Martha Langford's Suspended Conversations writes about the act of soldiers assembling photographic albums. Langford, approaches this from a photographic perspective, analyzing the albums themselves, while Winter writes about the war itself, speaking of photographs as a byproduct. ${ }^{18}$ Both texts were very relevant to my exploration of WWI advertisements.

The Mustering of Support for World War I by The Ladies Home Journal, by Joanne Karetzky was an interesting read. Speaking about the war from

15. Robert Jackall and Janice Hirota, Image Makers: Advertising, Public Relations, and the Ethos of Advocacy (Chicago: University of Chicago Press, 2000), 13.

16. Rob Kroes, Photographic Memories (Hanover, NH: Dartmouth College Press, 2007).

17. Tessa Morris-Suzuki The past within us : Media, memory, history (London; New York: Verso, 2005).

18. Martha Langford, Suspended conversations : The afterlife of memory in photographic albums (Ithaca: McGill-Queen's University Press, 2001), 67-76; Jay Winter, Remembering war: The great war between memory and history in the twentieth century (New Haven: Yale University Press, 2006), 80-102. 
the home front, Karetzky describes the Ladies Home Journal's transition from a peacetime magazine to a wartime one, including how this affected advertisers and their advertisements. ${ }^{19}$

In addition to books, I read many journal articles on subjects relevant to my research. Ultimately, the most helpful articles were ones that wove many of the elements in my analysis together. An excellent example of this is Sandy Callister's "Picturing Loss: Family, Photographs and the Great War". This article is an account of WWI from the perspective of New Zealanders. However, the information is also very relevant to the American's view of the war. Callister discusses Kodak's impact on the soldiers, their photographing as a result, and how these patterns shifted if a soldier lived or died in the war. ${ }^{20}$ The idea of photographing a soldier because he might die is one that I discuss in the analysis section, despite the fact that Kodak ads would never mention death.

Alan Trachtenberg's "Through a Glass, Darkly: Photography and Cultural Memory", while primarily an article about a photograph's representation of truth in the digital era, also discusses Civil War photography. I found that Trachtenberg's ideas about Gardner's Sketchbook of the War and its idealized cover image mirrored Kodak's portrayal of WWI. ${ }^{21}$ Conversely, Dannagal Goldthwaite Young's "Sacrifice, Consumption, and the American Way of Life: Advertising and Domestic Propaganda During

19. Joanne L. Karetzky, The Mustering of Support for World War I by The Ladies' Home Journal (Lewiston: Edwin Mellen Press, 1997).

20. Sandy Callister, "Picturing loss: Family, photographs and the Great War" Round Table 96, no. 393 (2007), 663-678.

21. Alan Trachtenberg, "Through a Glass, Darkly: Photography and Cultural Memory" Social Research 75, no. 1 (2008), 125-128. 
World War II" to be a counterpoint to the Civil War material, looking ahead (so to speak) to WWII and the different advertising strategy used in that time period..$^{22}$

"The Reframing of Traditional Cultural Values: Consumption and World War I", by Monica Brasted was helpful in my understanding of how advertising was used both before, and during WWI. In particular, it emphasized the emphasis on thrift most corporations worked into their advertisements during the war. ${ }^{23}$ In my analysis, I speak about how and why Kodak had no such emphasis. Additionally, Susan Zeiger's "She Didn't Raise Her Boy to be a Slacker: Motherhood, Conscription, and the Culture of the First World War" helped in my analysis of the wartime ads. It particularly helped in decoding the portrayal of women in these ads. ${ }^{24}$

The history of Kodak advertising, as stated, is not something that has been written about at length. The first source I looked at was Nancy Martha West's Kodak and the Lens of Nostalgia, which was published in 2000 , three years before the Kodak Advertising Collection came to the GEH. West's book is an excellent overview of the advertisements, offering history, specific examples, analysis of themes, and cultural history. West mentions in her

22. Dannagal Young, "Sacrifice, Consumption, and the American Way of Life: Advertising and Domestic Propaganda during World War II." Communication Review 8 , no. 1 (2005), 27-52.

23. Monica Brasted, "The Reframing of Traditional Cultural Values: Consumption and World War I." Advertising \& Society Review 5, no. 4 (2004), http://resolver.scholarsportal.info/resolve/15347311/v05i0004/nfp_trotcvcawwi (accessed July 26, 2010).

24. Susan Zeiger, "She Didn't Raise Her Boy to Be a Slacker: Motherhood, Conscription, and the Culture of the First World War". Feminist Studies 22, no. 1 (1996), 7-39, Academic Search Premier, EBSCOhost (accessed July 26, 2010). 
introduction eight topics that Kodak carries on "cultural conversations" with. These topics: "travel, automobility, childhood, toys, feminine beauty, family, memory, and World War $\mathrm{I}^{\prime 25}$ are very similar to the categories in which I have organized the advertisements in the finding aid. While West is regarded as the foremost scholar on the Kodak ads, it is important to note that her research was done before the collection moved to the GEH. As such, according to Kathy Connor, curator of the GEH who has helped West with projects since Kodak and the Lens of Nostalgia, it is important to understand that West was not able to see every ad when writing this book, and as such, some of the generalizations that she makes in the book have since been proven inaccurate ${ }^{26}$ However, this is not to say that West's book is not a great source of information. It is simply important to consider these more recent developments as well.

In addition to West's book, I have read Douglas Collins's The Story of Kodak. This book, published in 1990, gives a good overview of Kodak as a company. Besides a three-page section on the Kodak Girl, there is not much of interest in regards to the Kodak ads themselves contained within it, however it provided a good timeline of Kodak products when needed. Additionally, the George Eastman biography written by Elizabeth Brayer provided all the background on George Eastman himself that was needed.

Another vast source of Kodak materials is the department of Special Collections at the University of Rochester's Rush Rees Library. The U of R has

25. West, 3.

26. Kathy Connor, conversation with the author, 20 May 2010. 
received business papers from Eastman Kodak and I had hoped that some memos regarding ad production from my time period would survive.

However, a day spent scouring the boxes provided to me yielded little; it seems that there are no remaining behind-the-scenes memos from this time period.

While the archives in the GESC are extensive, it is prudent to mention that they are by no means the only collection of Kodak advertising material. Many other institutions hold impressive collections of Kodak materials as well. For example, Ryerson University holds the Kodak Collection, which was acquired in a similar manner to the GEH's acquisition of the Kodak Advertising Collection: In 2005, when Kodak Canada ceased operations at its Toronto location, arrangements were made for their corporate records, cameras, and the materials from their short-lived Heritage Museum to be transferred to the University. ${ }^{27}$ This collection includes Kodak Canada's advertising material, which was written about in Keri Lee Kauffman's thesis from 2009 "Kodak Canada: Increasing Access to Advertising Materials in Ryerson University Library's Special Collections". ${ }^{28}$ Similarly, The British Library holds the Kodak Limited's United Kingdom archives, donated to the Library in 2009. ${ }^{29}$

27. Ryerson University, Ryerson University Library - Special Collections http://www.ryerson.ca/library/collections/special/kodak.html (accessed August 29, 2010).

28. Keri Lee Kauffman, "Kodak Canada: Increasing Access to Advertising Materials in Ryerson University Library's Special Collections" (Masters Thesis, Ryerson University, 2009).

29. The British Library, "You press the button, we do the rest" http://www.bl.uk/news/2009/pressrelease20090318.html (accessed August 29, 2010). 
National holdings of Kodak collections can also be found in Australia at the Museum Victoria. ${ }^{30}$ Another significant holding of Kodak Ads is Duke University's "Ellis Collection of Kodakiana", a collection of advertisements from 1886-1923. This collection is particularly relevant because it is from the same time period as this thesis.

In addition to physical locations of Kodak materials, there are many online resources that can be consulted by a researcher. Many of the institutions that hold physical collections place materials online for access. The GESC has a large number of advertisements on their website available to the researcher ${ }^{31}$ Ryerson Library has placed a number of vintage Kodak publications on the website Archive.org. In fact, a search of Archive.org brings up over 500 mentions of Kodak, and there are many publications, and advertisements available for viewing. Lastly, there are also private collections that are accessible via an online presence. Websites like Kodakgirl.com, and Vintageadbrowser.com can be a vast source of ad material. For example, a search for Kodak on Vintageadbrowser.com produces 1170 ads, in many languages.

Finally, in preparation to create this finding aid, I believed it prudent to look at other examples of finding aids. I began with examples created by graduates of this program, looking at theses written by Kristin Dudley, Jennifer Givogue, and Lisa C. Hennessey. Each presented their information in

30. Museum Victoria, Browsing by theme 'Kodak Heritage Collection' Museum Victoria http://museumvictoria.com.au/collections/themes/2882/kodakheritage-collection (accessed August 29, 2010).

31. George Eastman House, Kodak Advertising Collection http://www.eastmanhouse.org/features/kodak-advertising/ (accessed August 29, 2010). 
slightly different ways, giving me many ideas to work with. None of these theses were created for the GESC, though, therefore I asked Kathy Connor to show me finding aids created especially for her material. In addition to these finding aids, I consulted both the Library of Congress's website as well as Duke University Library's, in order to understand their definition of a finding aid, as well as to view their online resources on the subject. 


\section{Methodology}

Knowing that I wanted to work with the Kodak advertising collection, I began by browsing the binders of ads, familiarizing myself with the scope and content of the collection as well as how it changed over the decades. The first decision was to choose when, out of the 104-year scope of the archive, I would focus on for the finding aid. Originally, the plan was to work only with the more image-based ads produced by Kodak. For the purposes of this thesis, I am defining image-based ads as the ones in which the focus is on the people in the advertisement and not the products. For example, an ad with a couple walking on the seaside accompanied by the text "Take a Kodak With You" would be an example of an image-based ad, while an ad featuring a photograph of a No. 1a Pocket Kodak Series II and featuring a slogan "A New Folding Pocket Kodak" would not. ${ }^{32}$ Ads such as this began in the early 1890 's, however they were not the primary means of advertising Kodak cameras at that time.

Ultimately, I decided to work with advertisements between 1900 and 1925. I chose this time period for a number of reasons: it covers both the early days of Kodak and World War I; George Eastman was still alive and thus still partially responsible for advertisements; sources indicate that during this time period Eastman Kodak dealt with the same advertising agency (Seaman Advertising Agency) and in-house advertising director

32. See Appendix A 
(Lewis Bunnell Jones) ${ }^{33}$; and lastly because this time period would offer an opportunity to study how Kodak's ads changed from the early text-dominated pieces to the more image-based ads they became known for.

As mentioned, this project began as an analysis geared exclusively to the image-based ads produced by Kodak. However, the realization that such an undertaking would also have to include the creation of a finding aid caused reevaluation of the material I was to survey. I understood that such a finding aid would be infinitely more useful if it included each advertisement in the binders, not just the image-based ads. Thus, I made the decision to include everything. The analysis of the image-based ads is still a part of this project however and therefore in the finding aid itself, advertisements are identified in part by their subject - this subject is either a category prevalent in the image-based ads, or a product.

When deciding what format my finding aid would take, I examined existing finder's tools in the GESC as well as The Richard and Ronay Menschel Library. While I understood the simplicity behind finder's tools that were paper based, I very much liked the versatility that a searchable spreadsheet supplied. ${ }^{34}$ I wanted my data to be searchable in a number of ways and so this seemed to be the ideal format. An Excel spreadsheet is versatile as well as easy to use. Additionally, it makes the process simple to import data into other, more complex database applications at a later time.

33. West, 24.

34. Lisa C. Hennessey, "The Mark A. Freeman Collection of World War II Photographs at George Eastman House" (master's thesis, Ryerson University, 2007). 
The next challenge was the question of what to index in the ads themselves. I decided that because the image-based ads were more complex and would be further analyzed, I would include additional criteria for those ads specifically. However, I wanted a single spreadsheet that I could streamline for either type of ad (either image-based or product based). The headings I decided on were: Binder, Year, Slogan, Subject(s), Text, Implied Audience, Product, Photo or Illustration, Color or Black and White, Publications, and an additional field for a description and other notes (Description/Notes). Of these eleven sections, four were eliminated for the straightforward product advertisements: Text, Implied Audience, Photo or Illustration, and Color or Black and White.

With headings established, I then began indexing the advertisements. In the twenty-five year period there were forty-three binders that were indexed. For the most part, duplicate advertisements were not counted, however this was not always the case. In some circumstances advertisements were identical with the exception of text, or imagery and so those ads would be counted.

Indexing the ads took approximately three and a half months. Considering the total contents of the Kodak Advertising Collection is 708 binders, it is easy to see why no finding aid at the individual ad level has been tackled before- it would take a enormous amount of time to complete. However, Kathy Connor, Curator of the GESC has indicated that with this twenty-five year period complete and able to be used as a template, she would like to solicit volunteers to begin working on the rest of the collection. 
The layout of the finding aid is formatted to be ever ready and capable of expansion. Being an Excel document, further headings could be added if needed for later ads. It is my hope that Connor achieves this goal, and perhaps future Photographic Preservation and Collections Management students make this finding aid a part of their Institutional Service Hours. While having twenty-five years of the collection indexed makes a difference in the availability of the collection to a researcher, having the entire collection completed would be phenomenal. 


\section{Analysis}

\section{Overview}

In total, the finding aid for the Kodak Advertising Collection consists of 2136 advertisements. Of these, 468 are image-based ads featuring a main subject that is not the product (and is almost always a person or group of people). As stated earlier, the majority of the time these ads are not selling a certain product, but are simply selling photography itself. All 468 ads can be placed into one of seven categories: women, children, family, farmers, travel and outdoors, labor, and soldiers. While an ad may belong to more than one category, and a category may target more than one type of person, every image-based ad counted in the finding aid can be slotted into one. The accompanying chart (fig 1) highlights how many ads feature each category, illustrating the popularity of each. In addition, examples of advertisements for each category can be found at the back of this thesis.

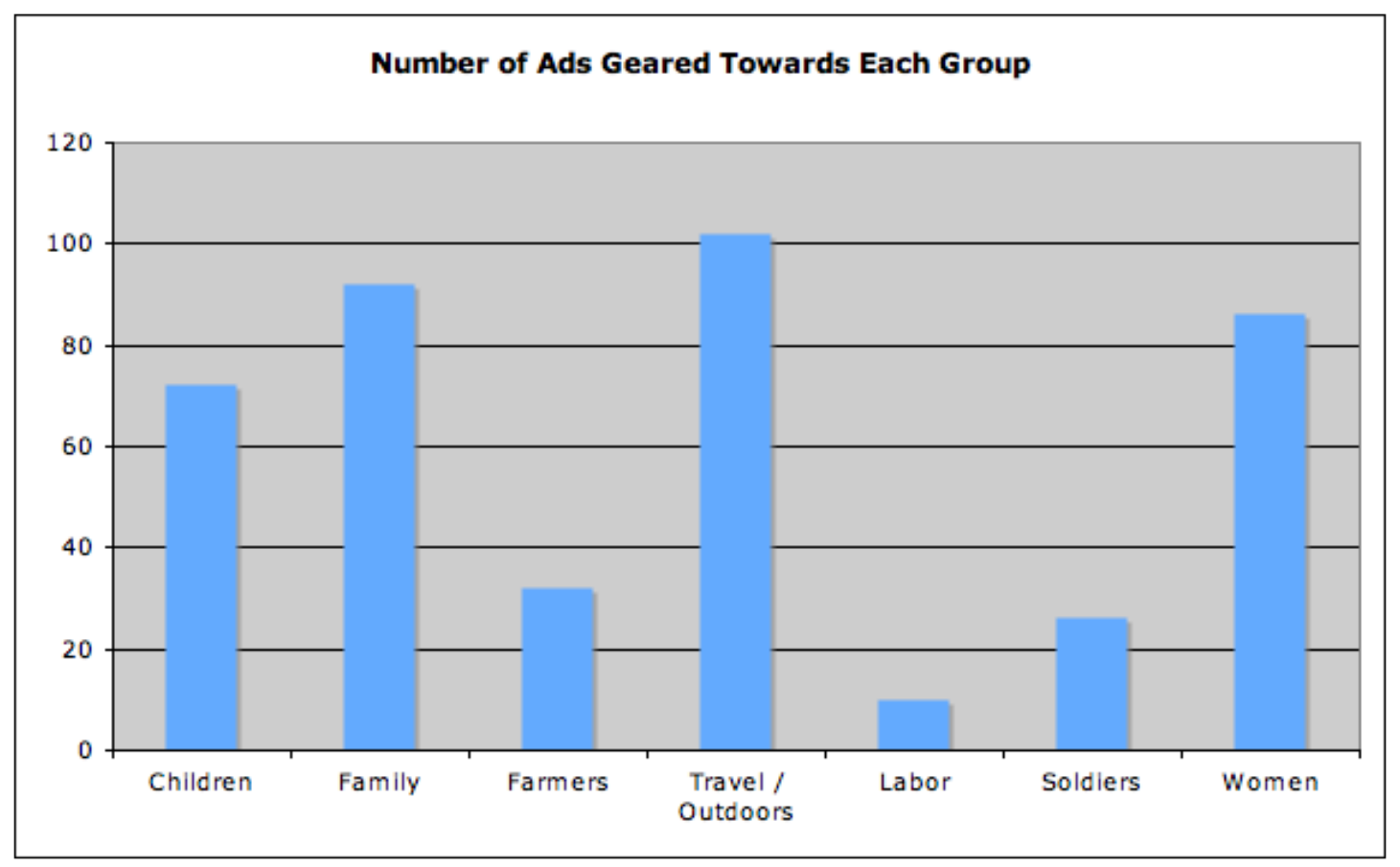

Figure 1 . The seven categories in the finding aid. 
Each of these categories was prevalent in advertisements of the time for many social and cultural reasons. It would be beyond the scope of this thesis to examine each category in depth, so I will choose one categorysoldiers - and examine the driving factors behind its inclusion in this list, deconstruct two pertinent examples of the advertisements from the collection, and discuss the ways in which Kodak used the image of soldiers and war in their campaigns.

Advertising and the Media in America During WWI

"The war has been won by advertising, as well as by soldiers and munitions," - such was the opinion of professional advertiser's magazine Printers Ink after the armistice. ${ }^{35}$ This sentiment seemed to be echoed by many, including George Eastman, who called advertisers "Cheerleaders of the Nation. ${ }^{\prime \prime 6}$ Wartime advertisements were influenced in part by the Committee on Public Information, which was formed by Woodrow Wilson at the beginning of the war to positively influence the opinions of Americans regarding the war. ${ }^{37}$ While this committee oversaw advertising and helped to create American propaganda, it was not responsible for the large-scale bans on many wartime photographs. These were mandated by the American Expeditionary Force, the branch of the military that accompanied journalists

35. Lears, 220.

36. Ibid., 222.

37. Jackall and Hirota, 13. 
and photographers. ${ }^{38}$ As West states, "No photos that could affect the morale of the American soldier, the public at home, or the United States relation with the Allied governments were allowed to be taken." ${ }^{139}$ These acts of propaganda and censorship combined to create an atmosphere of patriotism in America during the war. Unlike more recent wars, in which gruesome imagery from the front is beamed into ones home daily, contributing to an apathy or pacifist attitude felt by the American people, America in the grip of World War I was not entirely aware of the conditions faced by their men in the war- or, if they were, they pretended not to be. This perceived image of the war was quite idealized and of course was quite different from the reality of conditions the soldiers faced in Europe.

This view of war differs greatly with the American perception of the previous major war. The American Civil War, which took place only fiftytwo years prior to World War I, had been photographed in depth, and while the images were reliant on slow photographic processes, and therefore could not show the action of battle, they nevertheless showed the gruesome aftermath in great detail. Trachtenberg discusses the seminal Civil War photo book Gardner's Photographic Sketchbook of the Civil War in his paper, "Through a Glass, Darkly" He states that:

"Gardner directs the reading of the photographs as visual equivalents of victory, of 'union,' not simply in the sense of making a record of victory but by demanding in the act of interpretation how victory comes

38. West, 188.

39. Ibid., 189. 
about. ${ }^{\prime 40}$ Gardner's photographs show the realities of war, insofar as they include dead bodies, on both sides. While his book was not widely published, the photographs were well known and would have been part of the public consciousness by 1917 . However, the public image of World War I was more in line with the cover of Gardner's- an idyllic scene of a soldier's camp, with men socializing over a campfire, and no death or conflict. ${ }^{41}$ This scene is similar to the image of war taken up by Kodak, as will be shown in the analysis of individual advertisements.

Kodak, along with other advertisers, kept an optimistic tenor to their ads during the war years. Never mentioning death or risk and instead focusing on the separation felt by those left in America allowed Kodak to advertise the ways in which the feeling of separation could be lessened for the soldiers by sending them photographs.

Unlike other advertisements of the time, Kodak did not focus on the need for thrift and economy. Many corporations contributed to the wartime effort by encouraging their customers to be thrifty, for example advocating the conservation of food ${ }^{42}$ By doing this, these corporations helped the propaganda machine, while making themselves seem favourable to the American people by advertising their support for the war. Conversely, Kodak positioned their products as a form of morale for soldiers, encouraging not only the sending of photographs overseas, but even sending cameras for "the boys," thus urging the consumer to

34. Trachtenberg, 126.

41. Ibid.

42. Brasted. 
purchase quantities of film, paper, developing chemicals, etc. to keep up with the demand for these morale boosting photographs. Thriftiness was the message of other advertisers, whereas Kodak's message was to encourage patriotism by increasing communication to the soldiers "over there". Kodak was thus able to appear as patriotic as corporations advocating thrift without the economical hit. 
Analysis of Ads

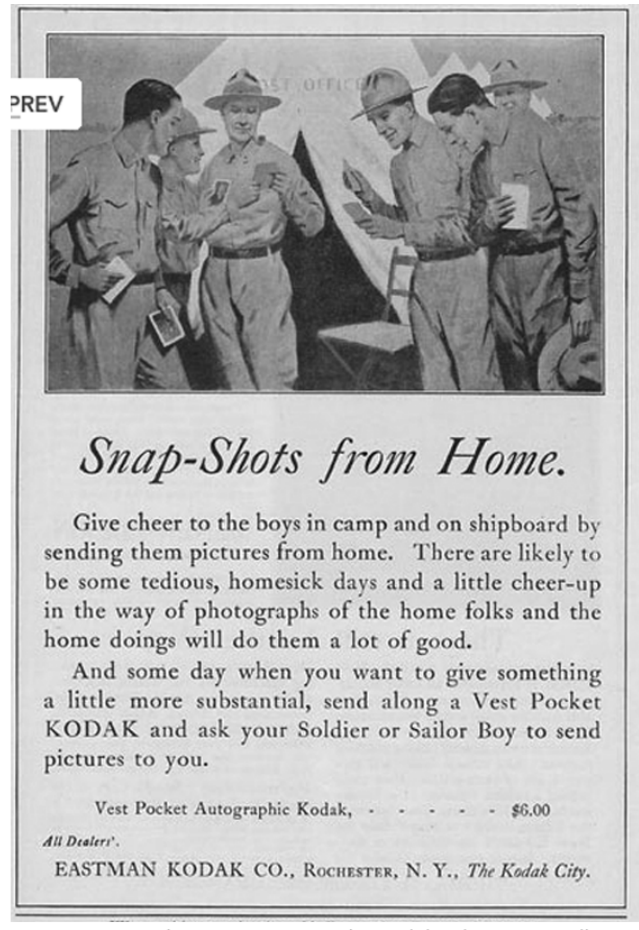

Figure 2. Snap-shots From Home. The Kodak Advertising Collec tion, The George Eastman Legacy Collection, George Eastman House.

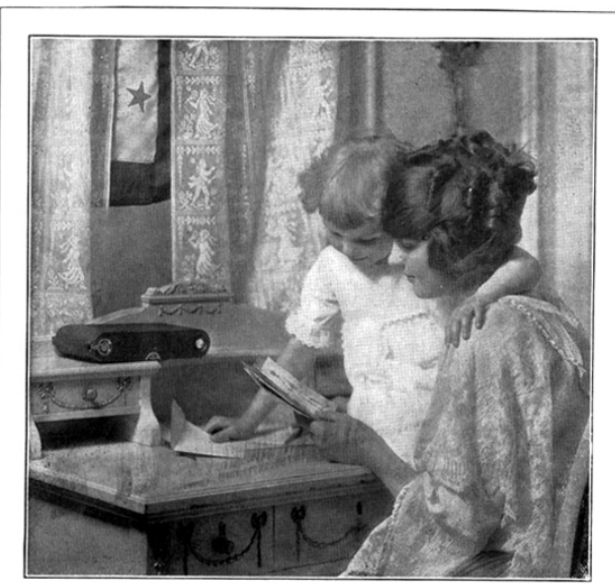

The Kodak Letter

cre."

The morning letter of cheer and hope has been written and with it pictures are going, simple Kodak pictures of their own taking that tell the home story, - pictures that will bring a cheery smile to his face, a leap of joy to his heart, that will keep bright the fire of courage in his soul as with the home image fresh in mind he battles for the safety of that home and for the honor of his flag.

EASTMAX KODAK CO., Rochester, N. Y., The Kodak City

Figure 3. The Kodak Letter. The Kodak Advertising Collection, The George Eastman Legacy Collection, George Eastman House.

Pictured above are two examples of Kodak advertisements during the war. These ads feature very different imagery and each will be analyzed in this section. The first advertisement, with the slogan "Snap-Shots from Home" features a group of soldiers gathered outside a tent, looking at photographs. All the men in the ad are smiling, clean cut, and dressed in their uniforms. The image is close cropped, leaving out all details of the camp besides the tent. The text in the ad implores you to "Give cheer to the boys in camp and on shipboard by sending them pictures from home. ${ }^{\prime 43}$ It goes on to note that "There are likely to be some tedious, homesick days and a little

43. Rochester, NY, George Eastman House George Eastman Legacy Collection, Kodak Advertising Collection, 1914-1916 5 of 5. It should be noted that this binder is mislabeled. It contains ads through 1917. 
cheer-up in the way of photographs from the home folks and the home doings will do them a lot of good. ${ }^{\prime 44}$ The image itself is non-photographic in origin. More than any other category in the collection, soldier ads are most often non-photographic in nature.

Despite the fact that the image supposedly depicts a war, there is no mention of battle in the ad copy. None of the soldiers in the image have guns or weapons of any kind on them. Additionally, none of the men even have insignia, rank, or identification of any kind on their uniforms. When looking at the advertisement, and ignoring the copy, what this ad resembles most are the ads for the Brownie Camera. Except, of course, in Brownie ads the people are all young boys. This ad concept- of a group of people looking at photographs is used many times for Brownie Cameras over the twenty-five year period I worked with. By emulating a Brownie ad that the consumer has likely seen before, Kodak is inferring that the men are simply enjoying a day outdoors. This juxtaposition of settings created a sense of peace in the ad that helps to set the viewer's mind at ease.

Joanne Karetzky emphasizes that the notion of an exchange of photographs with the troops is not one that was exclusive to Kodak. ${ }^{45}$ The cover of Ladies Home Journal for November 1918 featured a painting by Harold Brett entitled "His Thanksgiving" in which one soldier shows two others a photograph while they are standing in the trench, wearing helmets

44. Rochester, NY, George Eastman House George Eastman Legacy Collection, Kodak Advertising Collection, 1914-1916 5 of 5.

45. Karetzky, 26. 
and holding guns. ${ }^{46}$ However, the photograph the soldier is holding is a studio one, greatly resembling a cabinet card, not the snapshots Kodak would have people making. The sentiment is the same however- that the soldiers have little to remind them of home without the photographs sent from family.

The second advertisement, captioned "The Kodak Letter" features a mother and child writing a letter to a soldier. The star in the window- still a tradition in military families - is a powerful symbol that this small family is affected by the war. The mother and child review the photographs as the camera sits on the windowsill. This advertisement greatly resembles many family ads run by Kodak before and after the war. What makes this image different is the presence of the star in the window. Also unlike the first ad, the copy here mentions the war itself. The text reads:

"The Star in the window tells the story - their soldier is "over there" The morning letter of cheer and hope has been written and with it pictures are going, simple Kodak pictures that tell the home story, - pictures will bring a cheery smile to his face, a leap of joy to his heart, that will keep bright the fire of courage in his soul as with the home image fresh in mind as he battles for the safety of that home and for the honor of his flag. ${ }^{\prime \prime 7}$

West interprets this ad as creating an idealized image of a father, stating: "The stereotyped image of a state rather than of action transforms the soldier into the idealized father, and the family at home can 'remember' him

46. Karetzky, 26.

47. NY, George Eastman House George Eastman Legacy Collection, Kodak Advertising Collection, Amateur Ads 1917-1920 1 of 4. 
as such rather than as subject to the war's catastrophes. ${ }^{148}$ Additionally, according to Susan Zeiger, this ad is demonstrating the "good mother" stereotype ${ }^{49}$ : a woman who is the embodiment of a patriotic American. Zeiger compares this model to the stereotype of a "bad mother": a woman whose pre-disposition is to oppose the war due to motherly instincts, and feels the urge to keep boys close and therefore safe ${ }^{50}$ Zieger's notion that "Wartime mass media took on the responsibility of persuading women that public duty was the higher road"51 is evident in this ad. While the woman is not a mother to the soldier in question, she is, nonetheless, a mother and stands to lose greatly from this war. However, the ad depicts her proudly displaying a star, sending photographs overseas to keep spirits high, and effectually doing her part in the war.

Both ads, while different in subject matter, relate directly to soldiers. Both create an idealized picture of the war- the first shows the act of war itself, and the second shows the soldiers fighting it. With these wartime advertisements, Kodak then manages to sell cameras, teach the American public how to photograph and use photography during a war, and to contribute to the American propaganda machine. No small feat for two images and some text. However, what neither advertisement mentions is the real reason that photographs are so important during wartime: a soldier who goes to war stands a good chance of being killed. This was likely at the front of the minds of family members who frantically photographed soldiers as

48. West, 191.

49. Zeiger, 7.

50. Ibid., 5.

51. Ibid. 
they left their homes for the front, and also a driving reason to send cameras with them overseas, in order to capture their photographs for as long as possible. As Sandy Callister states: "What historians have overlooked is that, without bodies to grieve over, the medium of photography played an integral part in the representation of death and loss both during and after the war. Its role was both unanticipated and multivalent. ${ }^{152}$ However, it is not something that would have been overtly stated in advertisements at the time, for many reasons: of censorship, good taste, and because the notion of death is something Kodak does not address.

\section{WWI in Memory}

The scope and effect of World War I was so great that it was hard to comprehend. Despite this, Kodak still needed to sell cameras during wartime. Indeed, these cameras had a purpose: not only to capture home life for the soldiers, but also to give to the soldiers so that they in turn could photograph the war. West addresses this in her book, saying that the focus on the collection of war photos and the encouragement of this exchange of photographs both ways between soldier and family contributed to the domestication of the war: "Here war gets collected as a subject for the family album, an episode in the narrative of domestic life, presumably along with the wedding, the baby's first steps, the graduation, and family vacations.

52. Callister, 665. 
Kodak thus allows the war to be domesticated, in every sense of the word." ${ }^{53}$ In creating a space for the war in the family album, and thus the family history, Kodak makes the war itself seem more digestible. It now resides alongside other milestones, and thus is given rank with them. The war cannot be a big, incomprehensible event if it fits neatly into a family album. Thus, the act of photographing the war, in the eyes of Kodak, is a form of treatment- a means to make the situation make sense. As we will see, album making was a pastime taken up by many soldiers. This quaint Victorian parlor activity became an important act for many involved in the war.

The idea of placing these war photographs in context alongside other life events, or, conversely isolating them in their own albums is something explored by others as well. Martha Langford speaks more about photographs from WWI in family albums in her book Suspended Conversations. Langford analyses four WWI era albums: two from soldiers, and two from nurses. ${ }^{54}$ Like West, Langford comments on the placement of war photos among domestic scenes in the album. ${ }^{55}$ One of these albums is made by a soldier who died in combat and his album features photographs of friends and family interspersed with photographs of life at the army training camp. The album did not follow him to France, and thus ends when he leaves for the war. ${ }^{56}$ Langford also chronicles a young woman who was a nurse during the war. Her album was begun at the start of her service and includes not only

53. West, 193-196.

54. Langford, 67-76.

55. Ibid.

56. Ibid. 
snapshots, but also postcards, and newspaper clippings. At the conclusion of the war, this nurse continues to paste photos into the album of her later peacetime travels in Europe. "The extension of her wartime memoir into the twenties suggests that she viewed the war and its aftermath integrally, as a coming of age" ${ }^{157}$, Langford states, echoing West's "domestication" of the war. By defining the war as a coming of age experience, this nurse was labeling it, placing it among the other, definable points in her life. The album, with its easy transition, would help trick her memory in later years into thinking the war had been more manageable. Rob Kroes refers to photographs as a mnemonic device. He states that, "Revisiting old photographs, going over the family album is intended to trigger the reservoir of inner images, of memories" ${ }^{\prime 58}$ Thus, by integrating war images with peace ones, the albummaker assures happy recollections in the future. This idea is echoed in Callister's article. She describes soldier's albums thusly: "Soldierphotographers who did survive the war, and subsequently compiled their own war albums, generally structured their war stories in highly conventional ways. Many of them ended their war albums with photographs of domestic scenes depicting their transition from soldier to civilian. These endings are the stories of survivors. The messages they typically convey are of deliberate acts of closure. They declare affirmatively that, 'I am alive; the war is over, I am home again, back with my family, and I am looking forward to a future'." ${ }^{\prime 59}$

57. Langford, 73.

58. Kroes, 2.

59. Callister, 666. 
These photographs were also highly prized after the war as memories of loved ones who died. In her article, Callister writes on the fact that soldier's bodies did not return home. Oftentimes, the only part of a soldier the family would have left was his photograph. "During this war," she wrote, "Kodak did more than help reinforce the family's centrality to society and more than create new consumer needs and markets. It helped construct and preserve the family as a photographic memory and as a social unit. In too many instances photographs were the last visible traces a solider had of his family and his family of him. ${ }^{\prime 60}$ This association of photograph to memory, simplistically pitched to customers through advertisements during the war encompassed so much of the hopes and fears of soldiers and their families. Families desired photographs of their boys "over there" both to reassure themselves that the boys were alive and well, and to act as a surrogate if the worst should happen and the soldier die in the war. Conversely, soldiers craved photographs of their families to add to their albums and remind them of home. As Callister put it: "Kodak was ideally placed to grow both the soldier and the domestic market." ${ }^{61}$

For soldiers who survived, the Kodak advertisements during the war must have seemed like a parody of the war itself. The idealized version of war portrayed in the ads would have contrasted greatly with their assembled photo albums, which were assumedly not solely the merry images of camp that Kodak predicted. However, as Tessa Morris-Suzuki notes in her book The Past Within Us: "It is only when we look at our own face in the fading

60. Callister, 665.

61. Ibid. 
photograph that we understand how much we, and the world we live in, has altered with the passing of the years. The photograph album historicizes memory." Regardless if the photographs were pasted in a war album, or a family album beside a wedding, if the soldier lived or died- the Kodak ads contributed to the propaganda and patriotism of wartime in America, and influenced many soldiers and families to send loved ones a photo.

While one of the smallest categories in this period of Kodak advertisements, soldiers are one of the most unique. Created out necessity, this category completely overtook Kodak's advertising during the war years. The unique take on war, both at home and abroad, and its affect on American morale makes the wartime ads of Kodak a very interesting case study. 


\section{Conclusion}

The Kodak Advertising Collection is an important part of the George Eastman Study Center at the GEH. The 708 binders cover the history of the Eastman Kodak Company, until the 1980's making the collection integral to the study of Kodak advertisements. Current finding aids, while effective in narrowing down a search for advertisements within the collection, do not extend to the single ad level. With my creation of this finding aid, (focusing on the individual advertisements and recording relevant information such as slogans, subjects, target audience, years, and text), a new level of accessibility has been given to the years covered in this project.

The ads referenced in this project are an excellent window into what was happening both with Kodak and in the world at that time. We see children engaging with their surroundings as never before with the Brownie Camera, women dominating the scene with confidence and class holding the first movie camera for public use, and soldiers leaving for war with the Kodak Autographic Camera under their arm or their happy reunions with loved ones made possible through pictures taken with this Kodak. In the twenty-five year period this project covers, Kodak is there to evolve and succeed along with the people of the day, making their lives easier and more meaningful. It is the goal of this finding aid to make these ads more accessible because they still have plenty to offer us. They are an example of how Kodak firmly shaped the way we take photographs today, they allow us to smile at the notion of needing a reminder to bring a camera along on a vacation, and 
they give us a glimpse of nineteenth century life shot through the lens of a camera.

The creation of this finding aid will enable greater intellectual access to the Kodak Advertising Collection, while helping to preserve it for future researchers by cutting unnecessary access. It is my hope that the project is continued, following guidelines laid out in this paper, so that the collection can be made even more accessible. The material contained in this collection is rare and unique, and thus deserves to be preserved and indexed for future researchers. 


\section{Bibliography}

\section{History of Kodak:}

Brayer, Elizabeth. 2006. George Eastman : A biography. Rochester, NY: University of Rochester Press.

Collins, Douglas. 1990. The Story of Kodak. New York: H.N. Abrams.

Kay, T.. Selling an image: Interpreting gender in Eastman Kodak advertising, 1900--1915. M.A. diss., University of Wyoming, In Dissertations \& Theses: Full Text [database on-line]; available from http://www.proquest.com.ezproxy.lib.ryerson.ca (publication number AAT EP22395; accessed April 12, 2010

Kauffman, Keri Lee. 2009. Kodak Canada: Increasing Access to Advertising Materials in Ryerson University Library's Special Collections. Edited by Joint Graduate Program in Photographic Preservation and Collections Management.

West, Nancy Martha. 2000. Kodak and the Lens of Nostalgia. Charlottesville: University Press of Virginia,

\section{History of Advertising / History of America:}

Brasted, Monica. 2004. "The Reframing of Traditional Cultural Values: Consumption and World War I." Advertising \& Society Review 5, no. 4. http://resolver.scholarsportal.info/resolve/15347311/v05i0004/nfp_trot cvcawwi (accessed July 26, 2010).

Brennen, Bonnie, and Hanno Hardt. 1999. Picturing the Past : Media, history, and photography. The history of communication. Urbana: University of Illinois Press.

Callister, Sandy. 2007. "Picturing loss: Family, photographs and the Great War." Round Table 96, no. 393: 663-678. Academic Search Premier, EBSCOhost (accessed July 26, 2010).

Gold, Philip. 1987. Advertising, politics, and American culture : from salesmanship to therapy. New York: Paragon House.

Grant, Susan-Mary. 2005. "Raising the dead: war, memory and American national identity." Nations \& Nationalism 11, no. 4: 509-529. Academic Search Premier, EBSCOhost (accessed July 26, 2010).

Jackall, Robert, and Hirota, Janice M. 2000. Image Makers : advertising, public relations, and the ethos of advocacy. Chicago, IL: University of Chicago Press. 
Johnson, Paul. 1998; 1997. A History of the American People.1 US ed. New York, NY: HarperCollins Publishers.

Karetzky, Joanne L. 1997. The Mustering of Support for World War I by The Ladies' Home Journal. Lewiston: Edwin Mellen Press.

Kroes, Rob. 2007. Photographic Memories : Private pictures, public images, and american history. Interfaces, studies in visual culture. Hanover, N.H.: Dartmouth College Press : Published by University Press of New England.

Langford, Martha, and Musée McCord d'histoire canadienne. 2001. Suspended Conversations : The afterlife of memory in photographic albums. Montreal ; Ithaca: McGill-Queen's University Press.

Lears, T. J. Jackson. 1994. Fables of Abundance: A Cultural History of Advertising in America. New York: Basic Books.

Marchand, Roland. 1985. Advertising the American Dream: Making Way for Modernity, 1920-1940. Berkeley: University of California Press.

Morris-Suzuki, Tessa. 2005. The Past Within Us : Media, memory, history. London ; New York: Verso.

Reeves, Thomas C. 2000. Twentieth-Century America : a brief history. New York: Oxford University Press.

Trachtenberg, A.. "Through a Glass, Darkly: Photography and Cultural Memory." Social Research 75, no. 1 (April 1, 2008): 111-133. http://www.proquest.com.ezproxy.lib.ryerson.ca/ (accessed July 26, 2010).

Winter, J. M. 2006. Remembering War: The great war between memory and history in the twentieth century. New Haven: Yale University Press.

Young, Dannagal Goldthwaite. 2005. "Sacrifice, Consumption, and the American Way of Life: Advertising and Domestic Propaganda during World War II." Communication Review 8, no. 1: 27-52. Academic Search Premier, EBSCOhost (accessed July 26, 2010).

Zeiger, Susan. 1996. "She Didn't Raise Her Boy to Be a Slacker: Motherhood, Conscription, and the Culture of the First World War". Feminist Studies 22, no. 1, Women and the State in the Americas:7-39. Academic Search Premier, EBSCOhost (accessed July 26, 2010). 


\section{Finding Aids:}

Dudley, Kristin E. 2008. A Finding Aid for the Models' Guild of Philadelphia Collection. Edited by Louis Walton Sipley and Joint Graduate Program in Photographic Preservation and Collections Management.

Givogue, Jennifer. 2009. Finding Aid for the Set of George Streynsham Master's Albums at George Eastman House. Edited by Joint Graduate Program in Photographic Preservation and Collections Management.

Hennessey, Lisa C. 2007. The Mark A. Freeman Collection of World War II Photographs at George Eastman House. Edited by Joint Graduate Program in Photographic Preservation and Collections Management.

Library of Congress. "Finding Aids (Rare Book and Special Collections Reading Room, Library of Congress)." Library of Congress. http://www.loc.gov/rr/rarebook/findaid/findaid.html (accessed May 29, 2010). 


\section{Appendix A}

\section{Kodak Advertisement Examples}

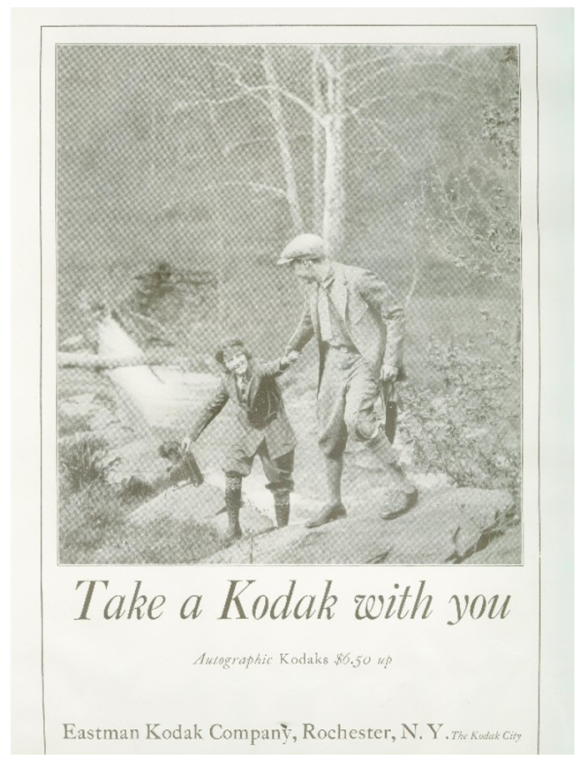

Figure 4. Take a Kodak with you. The Kodak Advertising Collection, The George Eastman Legacy Collection, George Eastman House.
Category: Travel / Outdoors

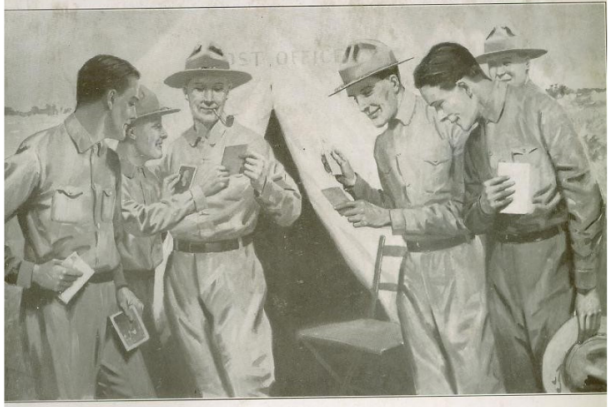

Snap-Shots from Home.

Give cheer to the boys in camp and on shipboard by sending them pictures from home. There are likely to be some tedious, homesick days and a little cheer-up in the way of photographs of the home folks and the home doings will do them a lot of good.

And some day when you want to give something a little more substantial, send along a Vest Pocket KODAK and ask your Soldieı or Sailor Boy to send pictures to you.

Figure 5. Snap-Shots from Home. The Kodak Advertising Collection, The George Eastman Legacy Collection, George Eastman House.

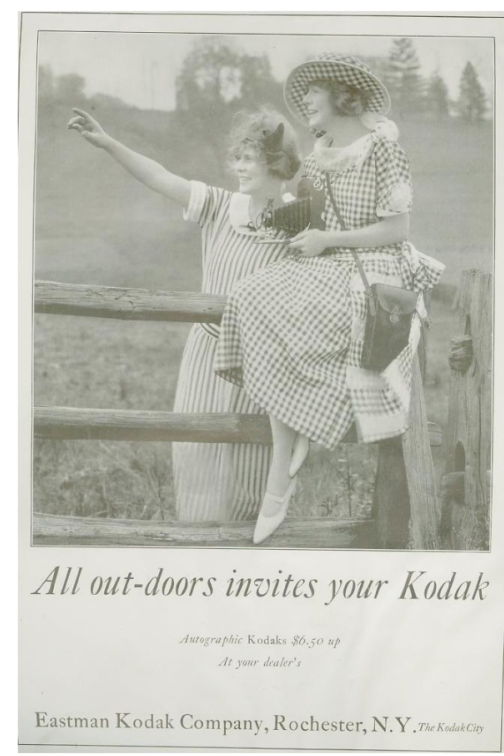

Figure 6. All out-doors invites your Kodak. The Kodak Advertising Collection, The George Eastman Legacy Collection, George Eastman House. Category: Women

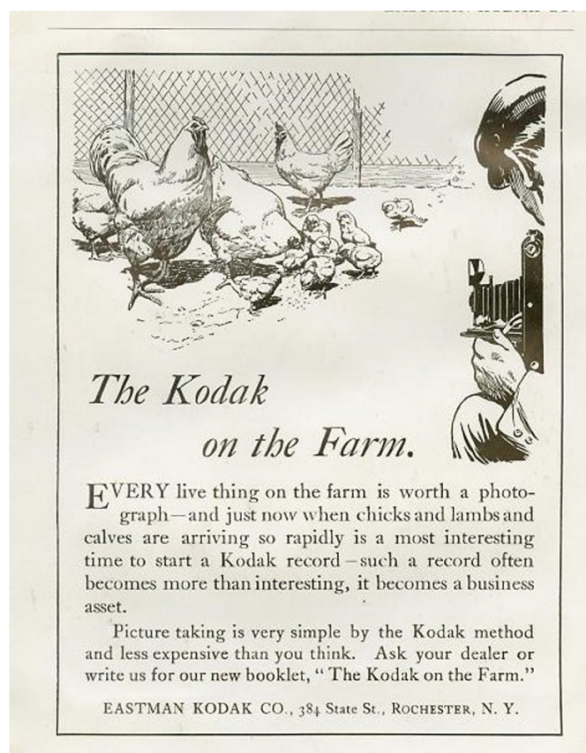

Figure 7. The Kodak on the Farm. The Kodak Advertising Collection, The George Eastman Legacy Collection, George Eastman House. 


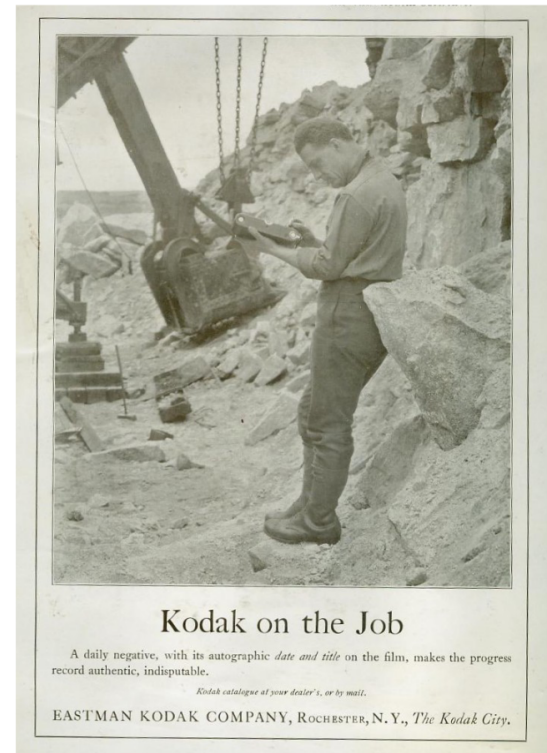

Figure 8. Kodak on the Job. The Kodak Advertising Collection, The George Eastman Legacy Collection, George Eastman House.

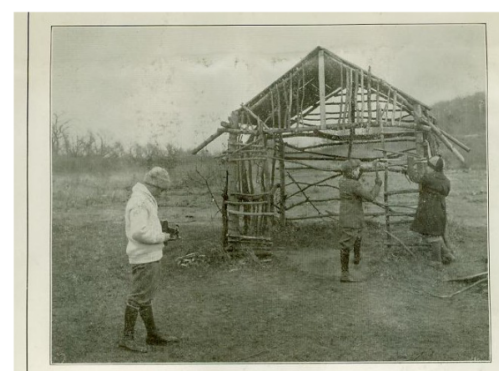

\section{Get it with a Brownie}

The shack that the bunch built offers a great chance for a picture. Get it with a Brownie.

Cross country hikes, sports of all sorts-your friends, your pets—all call for pictures that are easy to make the Brownie way.

No matter what the occasion there's always more fun with a Brownie along.

Brownie cameras \$2.00 up

Figure 9. Get it with a Brownie. The Kodak Advertising Collection, The George Eastman Legacy Collection, George Eastman House.

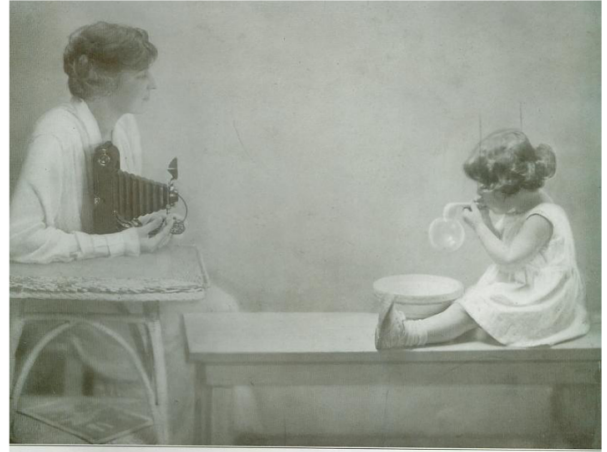

Keep a Kodak Story

of the Children.

And keep, as well, the date on the film, the authentic record lat tells how old Mary was when she took her first toddling steps ad the year and month when "brother" proudly donned his first ousers, or bravely set out for school with primer under his arm. Ich records are instantly and easily made and permanently kent Figure 10. Keep a Kodak Story of the Children. The Kodak Advertising Collection, The George Eastman Legacy Collection, George Eastman House. Category: Family

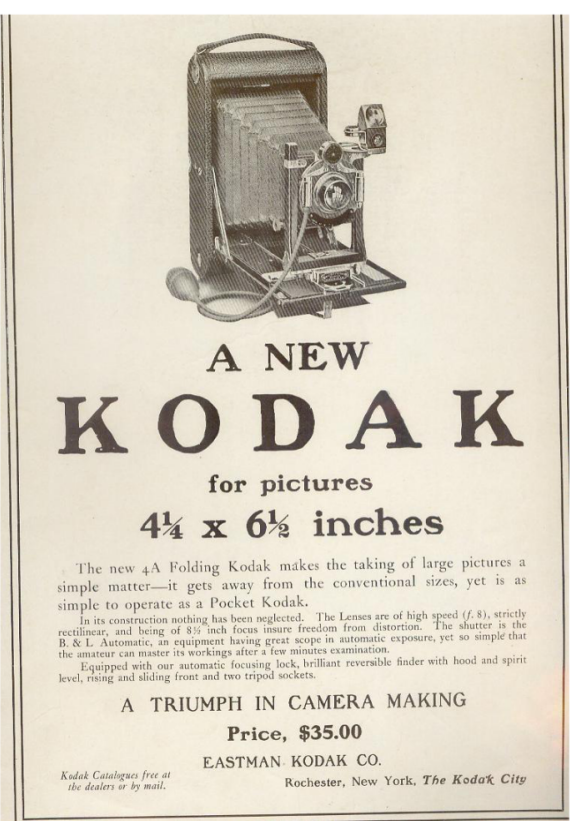

Figure 11. A New Kodak. The Kodak Advertising Collection, The George Eastman Legacy Collection, George Eastman House.
An example of a non-image based ad 
Appendix B

Finding Aid

Finding Aid : Kodak Advertising Collection

\section{Sarah Fay}

August 31, 2010 


\section{Descriptive Summary:}

\section{Creator}

Eastman Kodak Company.

\section{Extent}

39 Binders containing 2132 individual advertisements.

\section{Repository}

George Eastman Archives and Study Center.

\section{Language}

English. Some Spanish, French.

\section{Administrative Information:}

\section{Access Restrictions}

This collection is available for use by researchers, by appointment only. Please contact the George Eastman Archives and Study Centre to make an appointment. gesc@geh.org 585.271.3361 ext. 242/345.

\section{Use Restrictions}

Copyright of these advertisements remains with Eastman Kodak Company. Please speak with Kathy Connor, Curator of the GESC for more information.

\section{Preferred Citation}

Kodak Advertising Collection, George Eastman Legacy Collection, George Eastman House.

\section{Provenance}

Gift of the Eastman Kodak Company.

\section{Processing information}

Unknown.

\section{Collection Overview}

The Kodak Advertising Collection consists of 708 binders and scrapbooks. These are made up of several types of binders, as I am assuming availability changed over Kodak's history. For the twenty-five year 
period I surveyed, the binders measured 15" wide, $171 / 2$ " high and approximately $21 / 2$ " deep. Each binder contains between 35 and 50 pages that are slipped into plastic protective sheets. The ads themselves are pasted to black or white paper and are sometimes accompanied by penciled-in notes (most often the date). In some cases there is also a date stamped onto the ad, though this is not consistent. These books were compiled by the Eastman Kodak Company and were donated to the George Eastman House in 2003. The collection covers more than 100 years of advertisements- both tear sheets and printed ads- from 1884 to 1988. 
Binder: $1895-19073$ of 8

Year: 1901

Slogan: In Sealed Tubes

Subject(s): product - film \& paper

Product: "Blue Print" and Sepia Paper

Color or Black and White: bw

Description / Notes: article

Binder: $1895-19073$ of 8

Year: 1900

Slogan: Pocket Kodak Portraiture

Subject(s): product - camera

Product: Booklet

Color or Black and White: bw

Binder: $1895-19073$ of 8

Year: 1901

Slogan: "Pocket Kodak Portraiture"

Subject(s): product - camera

Product: Booklet

Color or Black and White: bw

Description / Notes: Article for a booklet featuring photos by

Rudolf Eickemeyer, Jr.

Binder: $1895-19073$ of 8

Year: 1901

Slogan: Kodak Possibilities

Subject(s): product - misc.

Product: Booklet

Color or Black and White: bw

Description / Notes: Kodaks price: $\$ 5.00$ to $\$ 35.00$

Binder: $1895-19073$ of 8

Year: 1903

Slogan: The Boy With A Brownie

Subject(s): boy

Text: Doubles his fun by taking pictures of his other sports. But

there's more than just fun in photography--there's instruction too.

No dark-room needed--all by daylight.
Implied Audience: parents/children

Product: Brownie

Photo or Illustration Based: photo

Color or Black and White: bw

Description / Notes: Photo of a boy holding a kite and a

Brownie.

Binder: $1895-19073$ of 8

Year: 1903

Slogan: The Boy with a Brownie

Subject(s): boy

Text: Doubles his vacation fun. Takes pictures of his sports and games and of the places of interest that he visits. But fun is not the only factor--there's education in photography. Any schoolboy of girl can make good pictures with a Brownie Camera--all without a dark-room and very inexpensively, now that the Kodak developing machine has added the finishing touch to the simplifying of photography.

Implied Audience: parents/children

Product: Brownie

Photo or Illustration Based: photo

Color or Black and White: bw

Description / Notes: Photo of a boy with a fishing rod, Brownie.

Binder: $1895-19073$ of 8

Year: 1902

Slogan: "It Works Like A Kodak"

Subject(s): five boys

Text: Any schoolboy or girl can make good pictures with one of the Eastman Kodak Company's No. 2 Brownie Cameras. These little instruments have fine meniscus lenses and our Rotary shutter for snap-shots and time exposures--the same shutter that is used on the famous Bullet and Bulls-eye Kodaks. They have sets of three stops and two finders one for upright and one for horizonta exposures. They are well made, have nickled fittings, are covered with imitation leather, and like Kodaks they use Film Cartridges and load in Daylight.

Implied Audience: parents/children

Product: Brownie 
Photo or Illustration Based: photo

Color or Black and White: bw

Description / Notes: Photo of five boys, on white looking at a Brownie.

Binder: $1895-19073$ of 8

Year: 1901

Slogan: Complete your vacation outfit with one of the Eastman

Dollar Brownie Cameras

Subject(s): product - camera

Product: Brownie

Description / Notes: Price: $\$ 1.00$

Binder: $1895-19073$ of 8

Year: 1901

Slogan: Interest the young folks

Subject(s): product - camera

Product: Brownie

Description / Notes: article

Binder: $1895-19073$ of 8

Year: 1900

Slogan: Eastman Kodak Co.'s Brownie Cameras

Subject(s): product - camera

Product: Brownie Camera

Description / Notes: Price: $\$ 1.00$

Binder: $1895-19073$ of 8

Year: 1900

Slogan: Any school-boy or girl can make good pictures with one of the Eastman Kodak Co.'s Brownie Cameras.

Subject(s): product - camera

Product: Brownie Camera

Description / Notes: Price: $\$ 1.00$

Binder: $1895-19073$ of 8

Year: 1900

Slogan: Fifteen of the Prize Winning Pictures

Subject(s): product - misc.
Product: Brownie Camera

Description / Notes: The Brownie Camera Club Contest

Binder: $1895-19073$ of 8

Year: 1901

Slogan: For Unusual Wants

Subject(s): product - camera

Product: Cartridge Kodaks

Description / Notes: article

Binder: $1895-19073$ of 8

Year: 1900

Slogan: Variety in tones on Dekko paper

Subject(s): product - film \& paper

Product: Dekko paper

Description / Notes: article

Binder: $1895-19073$ of 8

Year: 1902

Slogan: The "Just as Goods"

Subject(s): product - film \& paper

Product: Developers and toners

Color or Black and White:

Binder: $1895-19073$ of 8

Year: 1901

Slogan: "Double-Two" Film Cartridges

Subject(s): product - film \& paper

Product: Double-Two film cartridges

Description / Notes: article

Binder: $1895-19073$ of 8

Year: 1900

Slogan: Pin to a card and touch with a match

Subject(s): product - camera

Product: Eastman Flash Sheets

Description / Notes: Price per half dozen: $\$ 0.25$

Binder: $1895-19073$ of 8 
Year: 1902

Slogan: Eastman Folding Head Tripod

Subject(s): product - misc.

Product: Eastman Folding Head Tripod

Description / Notes: article.

Binder: $1895-19073$ of 8

Year: 1900

Slogan: The softness and delicacy of a fine etching can be more nearly approached by the use of Eastman's Royal Bromide Paper than by any other photographic process.

Subject(s): product - film \& paper

Product: Eastman Royal Bromide Paper

Color or Black and White:

Binder: $1895-19073$ of 8

Year: 1900

Slogan: Eastman's Permanent Bromide Paper

Subject(s): product - film \& paper

Product: Eastman's Permanent Bromide Paper

Color or Black and White:

Binder: $1895-19073$ of 8

Year: 1902

Slogan: After Fifteen years!

Subject(s): product - film \& paper

Product: Eastman's Permanent Bromide Paper

Color or Black and White:

Binder: $1895-19073$ of 8

Year: 1900

Slogan: Take a Kodak Home for Christmas

Subject(s): product - camera

Product: Folding Pocket Kodak

Description / Notes: Price: $\$ 10.00$ to $\$ 17.50$

Binder: $1895-19073$ of 8

Year: 1900

Slogan: The Full Charm of Winter Picture Taking is realized with a
Folding Pocket Kodak

Subject(s): product - camera

Product: Folding Pocket Kodak

Description / Notes: Kodaks Price: $\$ 5.00$ to $\$ 35.00$

Binder: $1895-19073$ of 8

Year: 1902

Slogan: A practical help in home portraiture

Subject(s): product - camera

Product: Folding Pocket Kodak

Description / Notes: article

Binder: $1895-19073$ of 8

Year: 1902

Slogan: The Difference between a folding Pocket Kodak

Subject(s): product - camera

Product: Folding Pocket Kodak

Description / Notes: Kodaks $\$ 5.00$ to $\$ 75.00$

Binder: $1895-19073$ of 8

Year: 1901

Slogan: Take a Kodak with you

Subject(s): Woman

Text: Made of aluminum and covered with the finest seal grain leather, the Folding Pocket Kodaks are as rich and dainty as a

lady's purse and are hardly heavier, yet they withstand the rough usage of travel and the changes of climate far better than any heavy camera. Carried in the hand, in a case, or in a shopping bag, they are convenient and inconspicuous. Take a Kodak with you. ...

Implied Audience: women

Product: Folding Pocket Kodak

Photo or Illustration Based: photo

Color or Black and White: bw

Description / Notes: Cut-out photo of a woman in a dress and hat with camera on her shoulder.

Binder: $1895-19073$ of 8

Year: 1901 
Slogan: Take a Kodak with you to the Pan American Exposition Subject(s): woman

Text: The Folding Pocket Kodaks are no trouble whether carried in the hand, in a case, or in the pocket, yet are instantly ready for use when wanted. They have the best lenses and shutters, and they load in daylight with our light-proof film cartridges.

Implied Audience: women

Product: Folding Pocket Kodak

Photo or Illustration Based: photo

Color or Black and White: bw

Description / Notes: Photo of the Kodak girl with parasol, Kodak in hand.

Binder: $1895-19073$ of 8

Year: 1901

Slogan: Take a Kodak with you

Subject(s): woman

Text: Like one's purse, a Folding Pocket Kodak may be carried in the hand without inconvience and being covered with fine seal

grain leather it is dainty and inconspicuous.

Implied Audience: women

Product: Folding Pocket Kodak

Photo or Illustration Based: photo

Color or Black and White: bw

Description / Notes: Photo of the Kodak girl, same as above.

Binder: $1895-19073$ of 8

Year: 1902

Slogan: The Folding Pocket Kodaks

Subject(s): woman

Text: Have the capabilities of cameras of triple their bulk. Though

of small compass they possess the very elements of successful

picture-taking--a simple mechanism and optical perfection.

Implied Audience: women

Product: Folding Pocket Kodak

Photo or Illustration Based: photo

Color or Black and White: bw

Description / Notes: Photo of the Kodak girl in white dress, holding a camera.
Binder: $1895-19073$ of 8

Year: 1901

Slogan: Complacency Upset

Subject(s): product - film \& paper

Product: Glass Plates

Description / Notes: article

Binder: $1895-19073$ of 8

Year: 1902

Slogan: There's fun for the young folks with a Brownie Camera

Subject(s): boy, two girls

Text: And it's fun that amounts to something, too--there's

education as well as amusement in photography. Any school boy or girl can make good pictures with a Brownie. Loads in daylight-develops in daylight too, if you use a Kodak developing machine.

Implied Audience: parents/children

Product: Kodak

Photo or Illustration Based: photo

Color or Black and White: bw

Description / Notes: Photo of a boy photographing two young girls. Caption reads "It works like a Kodak"

Binder: $1895-19073$ of 8

Year: 1901

Slogan: Take a Kodak with you to the Pan American

Subject(s): product - camera

Product: Kodak

Description / Notes: article

Binder: $1895-19073$ of 8

Year: 1904

Slogan: In War as in Peace The Kodak is at the front.

Subject(s): soldier

Text: In Cuba and the Philippines, in South Africa, in Venezuela and now in Korea and Manchuria, the camera most in evidence is the Kodak. The same qualities that make it indispensable to the correspondent make it most desirable to the tourist--simplicity, freedom fro dark room bother, lightness combined with a strength 
that resists the wear and tear of travel.

Implied Audience: travel / soldiers

Product: Kodak

Photo or Illustration Based: Illustration

Color or Black and White: bw

Description / Notes: Caption: "The Correspondent Drawn for

Eastman Kodak Co. By Frederic Remington Through Courtesy

Colliers Weekly. Copyright 1904 Collier's Weekly."

Binder: $1895-19073$ of 8

Year: 1901

Slogan: Take a Kodak with you

Subject(s): woman

Text: "Kodak" stands for all that is best in photography.

Implied Audience: women

Product: Kodak

Photo or Illustration Based: photo

Color or Black and White: bw

Description / Notes: Photo of the Kodak girl with parasol, Kodak

in hand. Caption reads: " The Kodak Girl"

Binder: $1895-19073$ of 8

Year: 1902

Slogan: Kodak Films

Subject(s): woman

Text: By reason of their non-halation quality and because of the great "latitude" which they give in exposure, produce better results than glass plates. Kodaks load in daylight--plate cameras require a dark room

Implied Audience: women

Product: Kodak

Photo or Illustration Based: photo

Color or Black and White: bw

Description / Notes: Photo of the Kodak Girl in a white dress, different pose again.

Binder: $1895-19073$ of 8

Year: 1902

Slogan: Kodak Simplicity
Subject(s): woman

Text: Has made the Kodak way the sure way in picture taking.

Implied Audience: women

Product: Kodak

Photo or Illustration Based: photo

Color or Black and White: bw

Description / Notes: Photo of a woman in a hat with Kodak on her shoulder.

Binder: $1895-19073$ of 8

Year: 1901

Slogan: A Kodak Christmas is the Merriest Christmas

Subject(s): woman

Text: Amid the festivities of Christmas-tide, one often finds the greatest charm of picture taking. The children, the children's tree, the visit at the old home, the flash-light at an evening gathering, the merry sleighing party, the home portraits of one's friends, all these offer subjects that have a personal interest, that one cherishes more highly as the years go by.

Implied Audience: women / family / Christmas

Product: Kodak

Photo or Illustration Based: photo

Color or Black and White: bw

Description / Notes: Photo of the Kodak Girl with camera in her hand.

Binder: $1895-19073$ of 8

Year: 1902

Slogan: The Dark-Room Abolished

Subject(s): woman

Text: Every step from loading the Kodak to finishing the prints

may now be accomplished without the aid of the darkroom. By means of the Kodak Developing Machine the amateur now

develops and fixes his own negatives in broad daylight and does it better than it was done before. The Kodak idea--simplicity-reaches its logical triumph in the new device. Anyone who can operate a Kodak (and who cannot?) can successfully operate the Kodak developing machine.

Implied Audience: women / general 
Product: Kodak

Photo or Illustration Based: photo

Color or Black and White: bw

Description / Notes: photo of a woman with a developing tank on the table.

Binder: $1895-19073$ of 8

\section{Year: 1903}

Slogan: A vacation without a Kodak is a vacation wasted.

Subject(s): woman

Text: No matter where you go or what your hobby may be.

Kodakery will add to the pleasure of your trip. Anybody can make good pictures by the Kodak System. It's all by daylight, now that the Kodak developing machine has abolished the darkroom

Implied Audience: women / travelers

Product: Kodak

Photo or Illustration Based: photo

Color or Black and White: bw

Description / Notes: Photo of the Kodak Girl with a Parasol.

Binder: $1895-19073$ of 8

Year: 1903

Slogan: Vacation means more if you Kodak

Subject(s): woman

Text: Not only all the joys that others have, but pictures besides.

Pictures of the cherished hobbies and of all the scenes that most

appeal to one. "Kodak" Stands for picture taking the easy way, for daylight development and better results.

Implied Audience: women / travelers

Product: Kodak

Photo or Illustration Based: photo

Color or Black and White: bw

Description / Notes: Photo of the Kodak Girl with a parasol.

Binder: $1895-19073$ of 8

Year: 1905

Slogan: The Kodak Baby Book

Subject(s): baby

Text: Tells in a non-technical way how to make home pictures of the little ones. Contains a dozen delightful Kodak studies of child life.

\section{Implied Audience: family}

Product: Kodak

Photo or Illustration Based: photo

Color or Black and White: bw

Description / Notes: Photo of a baby on a black BG.

Binder: $1895-19073$ of 8

Year: 1902

Slogan: Kodak Progress Competition

Subject(s): product - misc.

Product: Kodak and Brownie cameras

Description / Notes: Contest. 2,000 in cash, and 2,000 in prizes.

Binder: $1895-19073$ of 8

Year: April 1903

Slogan: Kodak Developing Machine

Subject(s): woman

Text: Do away absolutely with the dark-room and give better

results than the old way. Anybody can make perfect pictures by

the Kodak system. Ask your dealer to show you.

Implied Audience: women

Product: Kodak Developing Machines

Photo or Illustration Based: Illustration

Color or Black and White: bw

Publications: Cosmopolitan

Description / Notes: Illustration of a woman with the Kodak developing tank. Caption reads: "you press a button, then do the rest"

Binder: $1895-19073$ of 8

Year: 1900

Slogan: Eighty-Seven percent prefer film to Plates / For "Crisp"

Negatives.

Subject(s): product - film \& paper

Product: Kodak film

Color or Black and White: Publications: Photo Magazine

Description / Notes: article 
Binder: $1895-19073$ of 8

Year: 1902

Slogan: Kodak Success

Subject(s): product - film \& paper

Product: Kodak film

Color or Black and White:

Binder: $1895-19073$ of 8

Year: 1900

Slogan: A suggestion for framing photographs / Flash Sheets vs.

Flash Powders / Sun Lines on Film

Subject(s): product - misc.

Product: Kodak film

Color or Black and White: Publications: Photo Magazine

Description / Notes: article

Binder: $1895-19073$ of 8

Year: 1900

Slogan: A New Line of Kodak Albums

Subject(s): product - misc.

Product: Kodak film

Color or Black and White: Publications: Photo Magazine

Description / Notes: article

Binder: $1895-19073$ of 8

Year: 1900

Slogan: Kodak Films

Subject(s): product - film \& paper

Product: Kodak films

Color or Black and White:

Binder: $1895-19073$ of 8

Year: 1901

Slogan: Buy Photographic Paper in Original Packages Only /

Steam Development

Subject(s): product - film \& paper

Product: Kodak System

Description / Notes: article
Binder: $1895-19073$ of 8

Year: 1903

Slogan: The Kodak Way

Subject(s): women

Text: By the Kodak system every step in picture taking and

picture making is accomplished in daylight--loading, unloading, developing, fixing, printing. The Kodak way gives better results than the old way, too. Dark-Room Abolished.

Implied Audience: women / general

Product: Kodak System

Photo or Illustration Based: photo

Color or Black and White: bw

Description / Notes: Photo of a woman shooting a camera, and

then sitting at the table, developing. Caption reads: "you press a

button, then do the rest"

Binder: $1895-19073$ of 8

Year: 1901

Slogan: Kodaks for Serious Work / Kodak Portrait Attachments

Subject(s): product - camera

Product: Kodaks

Description / Notes: Article

Binder: $1895-19073$ of 8

Year: 1902

Slogan: Eastman's film negative albums

Subject(s): product - film \& paper

Product: Negative albums

Description / Notes: Article. Album price from $\$ 0.75$ to $\$ 1.50$

Binder: $1895-19073$ of 8

Year: 1901

Slogan: In the Panoramic Field

Subject(s): product - camera

Product: No. 1 Panoram-Kodak

Description / Notes: article. Caption reads: "Made with the No. 1 Panoram-Kodak 
Binder: $1895-19073$ of 8

Year: 1902

Slogan: Free Cameras For Teachers Disposal

Subject(s): product - camera

Product: No. 2 Brownie

Color or Black and White:

Binder: $1895-19073$ of 8

Slogan: It works like a Kodak. The Eastman Kodak Company's New No.2 Brownie Camera.

Subject(s): Young girl.

Text: Loads in daylight with six exposure film cartridges, has a fine meniscus lens with the Eastman Rotary shutter and set of three stops. It has two view finders, one for vertical and one for horizontal pictures, is well made in every particular, is covered with a fine quality imitation leather, has nickled fittings, and makes pictures $21 / 4$ by $31 / 4$ inches. Any school-boy or girl can make pictures with a Brownie Camera

Implied Audience: families

Product: No. 2 Brownie

Photo or Illustration Based: photo

Color or Black and White: bw

Description / Notes: Photo of a young girl with Brownie on a pile of books, photographing a toy.

Binder: $1895-19073$ of 8

Year: 1901

Slogan: The \$5.00 Falcon Camera

Subject(s): product - camera

Product: No. 2 Falcon Camera

Description / Notes: Mentions bicycle attachment and photo contest.

Binder: $1895-19073$ of 8

Year: 1900

Slogan: Folding Pocket Kodaks

Subject(s): product - camera

Product: No. 3 Folding Pocket Kodak

Description / Notes: Price: $\$ 17.50$
Binder: $1895-19073$ of 8

Year: 1901

Slogan: Special Lens and Shutter Equipments for Kodaks

Subject(s): product - camera

Product: No. 3 Folding Pocket Kodak

Description / Notes: Article.

Binder: $1895-19073$ of 8

Year: 1900

Slogan: Improvements in the Nos. 3, 4, and 5 Cartridge Kodaks

Subject(s): product - camera

Product: Nos. 3, 4, and 5 Cartridge Kodak

Description / Notes: article

Binder: $1895-19073$ of 8

Year: 1900

Slogan: The Eastman Products

Subject(s): product - misc.

Product: Papers, plates, tripods, cameras

Color or Black and White:

Binder: $1895-19073$ of 8

Year: 1902

Slogan: A Demonstration

Subject(s): product - film \& paper

Product: Solio

Color or Black and White:

Binder: $1895-19073$ of 8

Year: 1900

Slogan: Print from your vacation negatives on Velox

Subject(s): product - film \& paper

Product: Velox paper

Color or Black and White:

Binder: $1895-19073$ of 8

Year: 1900

Slogan: All days are good "printing days" to the photographer who 
used Velox

Subject(s): product - film \& paper

Product: Velox paper

Color or Black and White:

Binder: $1895-19073$ of 8

Year: 1900

Slogan: At home, With Velox

Subject(s): product - film \& paper

Product: Velox paper

Color or Black and White:

Binder: $1895-19073$ of 8

Year: 1901

Slogan: The Kodak Exhibit at the Pan American Exposition. Velox Demonstrations

Subject(s): product - film \& paper

Product: Velox paper

Description / Notes: article

Binder: $1895-19073$ of 8

Year: 1901

Slogan: Velox Prints in Any Light

Subject(s): product - film \& paper

Product: Velox paper

Color or Black and White:

Binder: $1895-19073$ of 8

Year: 1901

Slogan: Price List Eastman's W.D. Platinum Paper.

Subject(s): product - film \& paper

Product: WD platinum paper

Description / Notes: Article.

Binder: $1895-19073$ of 8

Year: 1900

Slogan: The 4x5 Weno Hawk-Eye

Subject(s): product - camera

Product: Weno Hawk-Eye
Description / Notes: Price: $\$ 8.00$

Binder: $1895-19073$ of 8

Year: 1900

Slogan: The Weno Hawk-Eye

Subject(s): product - camera

Product: Weno Hawk-Eye

Description / Notes: Price: $\$ 5.00$

Binder: $1895-19074$ of 8

Year: 1903

Slogan: Albuma

Subject(s): product - film \& paper

Product: Albuma paper

Color or Black and White:

Binder: $1895-19074$ of 8

Year: 1903

Slogan: Albuma Matchless in brilliancy and gloss

Subject(s): product - film \& paper

Product: Albuma paper

Color or Black and White

Binder: $1895-19074$ of 8

Year: 1904

Slogan: Photography for the Amateur at half its former cost

Subject(s): product - camera

Product: American Jr. Camera, Poco, Buck-Eye, American

Cameras.

Description / Notes: 4x5 Poco price: $\$ 4.80$ American Jr. Camera price: $\$ 1.60$

Binder: $1895-19074$ of 8

Year: 1903

Slogan: It's Brownie Camera Time

Subject(s): product - camera

Product: Brownie

Description / Notes: prices: No. 2 Brownies $\$ 2.00$ Film

Cartridges 12 exposures price: \$0.20 Brownie Developing Machine 
price: $\$ 2.00$, Brownie Developing Machine outfit price: $\$ 0.75$

Binder: $1895-19074$ of 8

Year: 1904

Slogan: There is Vacation Fun for every boy in a Brownie Camera

Subject(s): product - camera

Product: Brownie

Description / Notes: Price: $\$ 1.00$ to $\$ 9.00$

Binder: $1895-19074$ of 8

Year: 1904

Slogan: A Brownie Camera Means a Jolly Summer

Subject(s): product - camera

Product: Brownie Cameras

Description / Notes: Two Sizes: $\$ 1.00$ and $\$ 2.00$

Binder: $1895-19074$ of 8

Year: 1904

Slogan: Announcement.

Subject(s): product - film \& paper

Product: Collodion Paper

Description / Notes: letter about the amalgamation of the

Western Collodion paper company, Cedar Rapids into Kodak Park.

Binder: $1895-19074$ of 8

Year: 1904

Slogan: A Postal Gets

Subject(s): product - film \& paper

Product: Eastman Bromide Papers

Description / Notes: Mentions photo contest -"13 first prize medals"

Binder: $1895-19074$ of 8

Year: 1903

Slogan: As Long as the Paper lasts so long will the picture endure if it's on Eastman Permanent Bromide

Subject(s): product - film \& paper

Product: Eastman Permanent Bromide

Color or Black and White:
Binder: $1895-19074$ of 8

Year: 1902

Slogan: Thirteen years old, yet the chemical properties were perfect! Of course it was Eastman Permanent Bromide Paper.

Subject(s): product - film \& paper

Product: Eastman's Permanent Bromide Paper

Color or Black and White:

Binder: $1895-19074$ of 8

Year: 1904

Slogan: Enjoy Photography with little expense - Flexo Kodak $\$ 5.00$

Subject(s): product - camera

Product: Flexo Kodak

Description / Notes: Price: $\$ 5.00$

Binder: $1895-19074$ of 8

Year: 1902

Slogan: A New Folding Kodak

Subject(s): product - camera

Product: Folding Pocket Kodak

Description / Notes: No. 0 Folding Pocket Kodak price: $\$ 6.00$

Binder: $1895-19074$ of 8

Year: 1902

Slogan: A New Folding Pocket Kodak / The Judges

Subject(s): product - camera

Product: Folding Pocket Kodak

Description / Notes: article - mentions photo contests.

Binder: $1895-19074$ of 8

Year: 1902

Slogan: The success of the Folding Pocket Kodak

Subject(s): woman

Text: was only made possible by the Kodak film cartridge. Not only does the Kodak go inside the pocket, but inside the Kodak goes the film--all becomes one compact, self-contained

mechanism. Kodaks load in daylight--plate cameras require a 
darkroom. Kodak films, because of the great "latitude" which they give in exposure, produce better results than glass plates.

Implied Audience: women

Product: Folding Pocket Kodak

Photo or Illustration Based: photo

Color or Black and White: bw

Description / Notes: Photo of the Kodak Girl in a white dress with a camera in her hand.

Binder: 1895-1907 4 of 8

Year: 1904

Slogan: Built to Satisfy

Subject(s): product - camera

Product: Folding Pocket Kodaks / Kodets

Description / Notes: "23 styles of Kodaks and Kodets. $\$ 6.00$ to $\$ 100.00 "$

Binder: $1895-19074$ of 8

Year: 1903

Slogan: Folding Weno Hawk-Eye with Special Equipment

Subject(s): product - camera

Product: Folding Weno Hawk-Eye

Color or Black and White: Publications: Seaway

Description / Notes: No. 3 price: $\$ 22.50$ No. 4 price: $\$ 27.50$

Binder: $1895-19074$ of 8

Year: 1903

Slogan: You'll Enjoy photography most with a Folding Weno

Hawkeye

Subject(s): product - camera

Product: Folding Weno Hawk-Eye

Description / Notes: No. 3 Special Folding Weno: $\$ 15.00$ With Special Equipment: $\$ 22.50$. No. 4 Folding Weno: $\$ 22.50$. With

special equipment: $\$ 27.50$. Plate attachment: $\$ 3.00$

Binder: 1895-1907 4 of 8

Year: 1903

Slogan: Imperial Portrait Plates

Subject(s): product - film \& paper
Product: Imperial Portrait Plates

Color or Black and White:

Binder: $1895-19074$ of 8

Year: 1903

Slogan: The proof of Kloro is in the printing

Subject(s): product - film \& paper

Product: Kloro paper

Color or Black and White:

Binder: $1895-19074$ of 8

Year: 1902

Slogan: Tis Kodak Simplicity

Subject(s): woman

Text: and Kodak film convenience that have made pocket photography possible. Not only does the Kodak go inside the pocket, but inside the Kodak goes the film--all becomes one compact, self-contained mechanism.

Implied Audience: women

Product: Kodak

Photo or Illustration Based: photo

Color or Black and White: bw

Description / Notes: Photo of the Kodak girl in white dress. Same girl as above, different pose.

Binder: $1895-19074$ of 8

Year: 1903

Slogan: Demand Demonstration / Took Time and Snaps /

Skepticism Annihilated / Brownie Development Machine

Subject(s): product - darkroom

Product: Kodak / Brownie Developing Machine

Description / Notes: Article.

Binder: $1895-19074$ of 8

Year: 1903

Slogan: Photography without a Darkroom

Subject(s): Product: Kodak cameras, Developing machines

Description / Notes: prices: No. 2 Brownies $\$ 2.00$ Film

Cartridges 12 exposures price: $\$ 0.20$ Brownie Developing Machine 
price: $\$ 2.00$, Brownie Developing Machine outfit price: $\$ 0.75$

Binder: $1895-19074$ of 8

Year: 1903

Slogan: Kodak Correspondence School of Photography

Subject(s): product - misc.

Product: Kodak Correspondence School of Photography

Description / Notes: "Special instruction in: Exposure and

Development, Printing-Out Papers, Development Papers, Out-Door

Photography, Portraiture and Interiors, Platinum Printing, Bromide

Enlarging, Lantern Slide Making."

Binder: $1895-19074$ of 8

Year: 1903

Slogan: The Kodak Way

Subject(s): product - misc.

Product: Kodak Correspondence School of Photography

Description / Notes: "Tuition free to all owners of Kodak and

Brownie cameras upon payment of one dollar for text books.

Binder: $1895-19074$ of 8

Year: 1902

Slogan: The Darkroom Abolished

Subject(s): product - darkroom

Product: Kodak Developing Machine

Description / Notes: Price: 6.00

Binder: $1895-19074$ of 8

Year: 1902

Slogan: Skeptical? / The 4x5 Kodak Developing Machine

Subject(s): product - darkroom

Product: Kodak Developing Machine

Description / Notes: article

Binder: $1895-19074$ of 8

Year: 1902

Slogan: Enthusiastic / What Editor Todd Saw.

Subject(s): product - darkroom

Product: Kodak Developing Machine
Description / Notes: article.

Binder: $1895-19074$ of 8

Year: 1903

Slogan: You Press the Button-- then do the rest

Subject(s): product - darkroom

Product: Kodak Developing Machine

Color or Black and White

Binder: $1895-19074$ of 8

Year: 1903

Slogan: No Darkroom

Subject(s): product - darkroom

Product: Kodak Developing Machine

Color or Black and White:

Binder: $1895-19074$ of 8

Year: 1903

Slogan: The Evidence

Subject(s): product - darkroom

Product: Kodak Developing Machine

Description / Notes: article.

Binder: $1895-19074$ of 8

Year: 1903

Slogan: Illusions Dispelled

Subject(s): product - darkroom

Product: Kodak Developing Machine

Description / Notes: article.

Binder: $1895-19074$ of 8

Year: 1904

Slogan: Now Ready

Subject(s): product - darkroom

Product: Kodak Developing Machine

Description / Notes: article

Binder: $1895-19074$ of 8

Year: April 1903 
Slogan: Kodak Developing Machine

Subject(s): product - darkroom

Product: Kodak Developing Machine

Description / Notes: Price for developing machines: $\$ 2.00$ $\$ 10.00$

Binder: $1895-19074$ of 8

Year: 1904

Slogan: The Kodaker Abroad

Subject(s): product - film \& paper

Product: Kodak film

Description / Notes: List of Kodak dealers in Europe and Australia

Binder: $1895-19074$ of 8

Year: 1902

Slogan: What they said and what happened

Subject(s): product - film \& paper

Product: Kodak Films

Description / Notes: article.

Binder: $1895-19074$ of 8

Year: 1903

Slogan: An Ingredient

Subject(s): product - film \& paper

Product: Kodak Films

Color or Black and White:

Binder: $1895-19074$ of 8

Year: 1904

Slogan: Handling N.C. Film / Converted to Film

Subject(s): product - film \& paper

Product: Kodak Films

Description / Notes: article.

Binder: $1895-19074$ of 8

Year: 1902

Slogan: Kodak Films

Subject(s): woman
Text: The largest clock keeps no more perfect time than a pocket chronometer: the largest camera makes no more perfect picture than a pocket Kodak--'tis the Kodak quality. Not only does the Kodak go inside the pocket but inside the Kodak goes the film--all becomes one compact, self-contained mechanism. By reason of their non-halation quality and because of the great "latitude" which they give in exposure, produce better results than glass plates. Kodaks load in daylight--plate cameras require a darkroom.

Implied Audience: women / general

Product: Kodak films

Photo or Illustration Based: photo

Color or Black and White: bw

Description / Notes: Photo of the Kodak girl in a white dress and hat with flowers, holding a Kodak.

Binder: $1895-19074$ of 8

Year: 1904

Slogan: Start Right / Photographic Competitions

Subject(s): product - darkroom

Product: Kodak Films, developing machine

Description / Notes: Includes a list of the important photographic contests in the United States

Binder: $1895-19074$ of 8

Year: 1902

Slogan: Rapid Rectilinear Lens

Subject(s): product - camera

Product: Kodak Lenses

Description / Notes: article

Binder: $1895-19074$ of 8

Year: 1903

Slogan: Anybody can make good pictures by the Kodak System

Subject(s): product - camera

Product: Kodak System

Color or Black and White:

Binder: $1895-19074$ of 8

Year: 1904 
Slogan: Kodak Quality

Subject(s): product - camera

Product: Kodaks

Description / Notes: Kodaks, Kodets, Bullets, price: $\$ 6.00$ to $\$ 100.00$

Binder: $1895-19074$ of 8

Year: 1904

Slogan: Eastman's Kodoid Plates

Subject(s): product - film \& paper

Product: Kodoid Plates

Description / Notes: Article.

Binder: $1895-19074$ of 8

Year: 1902

Slogan: The Focusing Weno

Subject(s): product - camera

Product: No. 3 Focusing Weno Hawk-Eye No. 4 Focusing Weno

Hawk-Eye

Description / Notes: No. 3 Focusing Weno Hawk-Eye price:

$\$ 27.50$ No. 4 Focusing Weno Hawk-Eye Price: $\$ 30.00$

Binder: $1895-19074$ of 8

Year: 1902

Slogan: A New Plate Camera

Subject(s): product - camera

Product: No. 5 Eastman Plate Camera

Description / Notes: Article.

Binder: $1895-19074$ of 8

Year: 1903

Slogan: All the qualities you want in a plate--not merely some of them. Orthonon

Subject(s): product - film \& paper

Product: Orthonon Plates.

Color or Black and White:

Binder: $1895-19074$ of 8

Year: 1902
Slogan: Kodak Progress Competition

Subject(s): product - misc.

Product: photo contest

Description / Notes: $\$ 4,000$ in prizes, judged by Rudolf

Eickemeyer Jr., Charles I. Berg, Henry Troth.

Binder: $1895-19074$ of 8

Year: 1904

Slogan: The Pocket Kodaks

Subject(s): product - camera

Product: Pocket Kodak

Description / Notes: Price with a roll of film: $\$ 5.00$

Binder: $1895-19074$ of 8

Year: 1903

Slogan: Facts about Solio

Subject(s): product - film \& paper

Product: Solio Paper

Color or Black and White:

Binder: $1895-19074$ of 8

Year: 1904

Slogan: Solio is an honest paper

Subject(s): product - film \& paper

Product: Solio Paper

Color or Black and White:

Binder: $1895-19074$ of 8

Year: 1904

Slogan: The Star Premo

Subject(s): product - camera

Product: Star Premo

Description / Notes: Star Premo Price: $\$ 25.00$. Film Pack Adaptor: $\$ 1.00$. Premo Film Pack: $\$ 0.70$

Binder: $1895-19074$ of 8

Year: 1904

Slogan: $\$ 8.00$ One Button Does it.

Subject(s): product - camera 
Product: The Bullet

Description / Notes: Price: $\$ 8.00$

Binder: 1895-1907 4 of 8

Year: 1904

Slogan: Bullets

Subject(s): product - camera

Product: The Bullet

Description / Notes: Price $\$ 8.00$

Binder: 1895-1907 4 of 8

Year: 1903

Slogan: In any light

Subject(s): product - camera

Product: The Kodak System

Color or Black and White:

Binder: 1895-1907 4 of 8

Year: 1902

Slogan: Velox will give you the most satisfactory results from your vacation negatives.

Subject(s): product - film \& paper

Product: Velox

Color or Black and White:

Binder: $1895-19074$ of 8

Year: 1903

Slogan: Use double weight Special Rough Velox for your best work

Subject(s): product - film \& paper

Product: Velox

Color or Black and White:

Binder: 1895-1907 4 of 8

Year: 1902

Slogan: At home, With Velox

Subject(s): Woman

Text: By the light of an ordinary gas jet, by lamplight or by

daylight, print making is easy with Velox. Velox yields prints of the softest and richest quality, giving deep, pure blacks, and mellow highlights. No dark room required. Simplest for the amateur.

Implied Audience: women

Product: Velox

Photo or Illustration Based: photo

Color or Black and White: bw

Description / Notes: Photo of a woman holding a printing frame up to a light.

Binder: $1895-19074$ of 8

Year: 1904

Slogan: Velox Liquid Developer

Subject(s): product - film \& paper

Product: Velox Liquid Developer

Color or Black and White:

Binder: $1895-19074$ of 8

Year: 1904

Slogan: No Abrasion

Subject(s): product - film \& paper

Product: Velox Liquid Developer

Color or Black and White: Publications: Photographer

Binder: $1895-19074$ of 8

Year: 1903

Slogan: An Evening with Velox

Subject(s): product - film \& paper

Product: Velox paper

Color or Black and White:

Binder: $1895-19074$ of 8

Year: 1902

Slogan: The Dark-Room Abolished

Subject(s): woman

Product: Color or Black and White:

Binder: $1895-19075$ of 8

Year: 1904

Slogan: The New 3A Folding Pocket Kodaks 
Subject(s): product - camera

Product: 3A Folding Pocket Kodak

Description / Notes: Price: $\$ 20.00$

Binder: $1895-19075$ of 8

Year: 1904

Slogan: Albuma

Subject(s): product - film \& paper

Product: Albuma

Color or Black and White:

Binder: $1895-19075$ of 8

Year: 1904

Slogan: Albuma

Subject(s): product - film \& paper

Product: Albuma

Color or Black and White:

Binder: $1895-19075$ of 8

Year: 1904

Slogan: American Jr. Camera

Subject(s): product - camera

Product: American Jr.

Description / Notes: Price: $\$ 1.60$

Binder: $1895-19075$ of 8

Slogan: Vacation fun Doubles when you have a Brownie Camera.

Subject(s): camera

Text: Pictures of everything you are interested in at small cost and no trouble.

Implied Audience: travel

Product: Brownie

Photo or Illustration Based: illus

Color or Black and White: BW

Description / Notes: 1.002 .005 .00

Binder: 1895-1907 5 of 8

Slogan: An Eastman for a An Eastman for a Dollar: The No. 1 Brownie Camera
Subject(s): product - camera

Text: It works like a Kodak.

Product: Brownie

Photo or Illustration Based: illus

Color or Black and White: BW

Description / Notes: small

Binder: 1895-1907 5 of 8

Slogan: The No. 2 Brownie Camera

Subject(s): product - camera

Text: Photograph your friends - and the places you visit. ... is

simple in operation and gives excellent results.

Product: Brownie

Photo or Illustration Based: illus

Color or Black and White: BW

Binder: $1895-19075$ of 8

Year: 1904

Slogan: It works like a Kodak. A One or Two-Dollar Brownie

Camera

Subject(s): product - camera

Product: Brownie

Description / Notes: Price: $\$ 1 \$ 2$

Binder: $1895-19075$ of 8

Slogan: A one or two dollar Brownie Camera

Subject(s): Text: Text: Will double summer fun of any wideawake boy or girl. Pictures of vacation haunts are well worth preserving, and photography is now so simple that anybody can make good pictures.

Implied Audience: parents/children

Product: Brownie

Photo or Illustration Based: Text: Color or Black and White: BW

Binder: $1895-19075$ of 8

Year: 1904

Slogan: A Christmas Suggestion: Buck-Eye

Subject(s): product - camera 
Product: Buck-Eye

Description / Notes: Price: $\$ 2.50$ to $\$ 18.00$

Binder: $1895-19075$ of 8

Year: 1940

Slogan: The Odyssey of a Camera Man

Subject(s): Product: cameras

Description / Notes: Article about a news photographer "The

Odyssey of a Camera Man"

Binder: $1895-19075$ of 8

Year: 1904

Slogan: Kodak Developing Machine

Subject(s): product - darkroom

Product: Developing Machine

Description / Notes: Developing Machine Price: $\$ 2.00$ to $\$ 10.00$.

Kodaks price: $\$ 5.00$ to $\$ 75.00$

Binder: 1895-1907 5 of 8

Year: 1904

Slogan: Imperial Portrait Plates

Subject(s): product - film \& paper

Product: Dry Plates

Color or Black and White:

Binder: 1895-1907 5 of 8

Year: 1904

Slogan: Two special qualities in one plate, Orthonon

Subject(s): product - film \& paper

Product: Dry Plates

Color or Black and White:

Binder: 1895-1907 5 of 8

Year: 1904

Slogan: Standard Extra

Subject(s): product - film \& paper

Product: Dry Plates

Color or Black and White:
Binder: 1895-1907 5 of 8

Year: 1904

Slogan: Standard Extra

Subject(s): product - film \& paper

Product: Dry Plates

Color or Black and White:

Binder: 1895-1907 5 of 8

Year: 1904

Slogan: Flash a Flash with an Eastman Flash Sheet

Subject(s): product - camera

Product: Eastman Flash Sheet

Description / Notes: Price per pack: $\$ 0.25, \$ 0.40, \$ 0.60$

Binder: 1895-1907 5 of 8

Year: 1904

Slogan: Just a Second!

Subject(s): product - camera

Product: Eastman Flash Sheet

Color or Black and White:

Binder: $1895-19075$ of 8

Year: 1904

Slogan: Flashlight Time

Subject(s): product - camera

Product: Flash Sheets

Description / Notes: Price: No. 1 half dozen sheets $\$ 0.25$ No. 2 : $\$ 0.40$ No. $3 \$ 0.50$

Binder: 1895-1907 5 of 8

Slogan: The Flexo Kodak

Subject(s): product - camera

Text: Price 5.00 Its simplicity commends it to beginners in

photography. Its capability - to the experienced.

Product: Flexo Kodak

Photo or Illustration Based: Illus

Color or Black and White: BW

Binder: $1895-19075$ of 8 
Slogan: Flexo Kodak : Price $\$ 5$

Subject(s): product - camera

Text: Its simplicity commends itself to beginners in photography.

It's capability-to the experienced.

Product: Flexo Kodak

Photo or Illustration Based: Illus

Color or Black and White: BW

Binder: $1895-19075$ of 8

Slogan: The Flexo

Subject(s): product - camera

Text: Price, $5.00 \ldots$ it has that Kodak simplicity which is of so

much importance to the beginner = yet it lacks none of the

essentials to good picture making. ...

Product: Flexo Kodak

Photo or Illustration Based: illus

Color or Black and White: BW

Binder: $1895-19075$ of 8

Year: 1904

Slogan: Flexo Kodak Price $\$ 5$.

Subject(s): product - camera

Product: Flexo Kodak

Description / Notes: Price: \$5.00. Loads 2,4,6, or 12 exposures

Binder: $1895-19075$ of 8

Year: 1904

Slogan: The Folding Buck-Eye

Subject(s): product - camera

Product: Folding Buck-Eye

Description / Notes: Price: $\$ 7.50$

Binder: $1895-19075$ of 8

Slogan: A New Folding Pocket Kodak

Subject(s): product - camera

Text: The No. 3a Broader in scope than anything heretofore

attained in pocket photography

Product: Folding Pocket Kodak

Photo or Illustration Based: illustration

\section{Color or Black and White: BW}

Binder: $1895-19075$ of 8

Slogan: Increased Possibilities. All the Old Simplicity.

Subject(s): product - camera

Text: The New Model No. 3 Folding Pocket Kodaks (small) have

automatic shutters with pneumatic release, and high speed lenses.

Product: Folding Pocket Kodak

Photo or Illustration Based: Illus

Color or Black and White: BW

Binder: $1895-19075$ of 8

Slogan: The Highest Achievement in Pocket Photography

Subject(s): product - camera

Text: New Model No3 Folding Pocket Kodak Marked Improvement,

But No change in Price.

Product: Folding Pocket Kodak

Photo or Illustration Based: illustration

Color or Black and White: BW

Binder: $1895-19075$ of 8

Slogan: Keeping Step with the Kodak Progress

Subject(s): product - camera

Text: is the new model of the No 2 Folding Pocket Kodak which is equipped with

Product: Folding Pocket Kodak

Photo or Illustration Based: illus

Color or Black and White: BW

Description / Notes: price 15.00

Binder: $1895-19075$ of 8

Slogan: If it isn't an Eastman, it isn't a Kodak

Subject(s): product - camera

Text: The New 3a folding pocket Kodaks.... Photography means

Kodakery with the bother left out, means daylight development,

fewer chemicals, better pictures.

Product: Folding Pocket Kodak

Photo or Illustration Based: Illus

Color or Black and White: BW 
Description / Notes: price 20.00

Binder: $1895-19075$ of 8

Year: 1904

Slogan: Folding Pocket Kodak

Subject(s): product - camera

Product: Folding Pocket Kodak

Description / Notes: Price: $\$ 6.00$ to $\$ 20.00$

Binder: $1895-19075$ of 8

Year: 1904

Slogan: $\$ 6$ to $\$ 20$. Folding Pocket Kodaks

Subject(s): product - camera

Product: Folding Pocket Kodak

Description / Notes: Price: $\$ 6.00$ to $\$ 20.00$

Binder: $1895-19075$ of 8

Year: 1904

Slogan: The Folding Hawk-Eye

Subject(s): product - camera

Product: Hawk-Eye No. 3 and No. 4

Description / Notes: No. 3 Folding Hawk-Eye: $\$ 22.50$ No. 4

Folding Hawk-Eye: $\$ 27.50$ Glass plate attachment: $\$ 2.50$

Binder: $1895-19075$ of 8

Year: 1904

Slogan: Kloro The Insoluble Emulsion Paper

Subject(s): product - film \& paper

Product: Kloro

Color or Black and White:

Binder: $1895-19075$ of 8

Year: 1904

Slogan: Kloro

Subject(s): product - film \& paper

Product: Kloro

Color or Black and White:

Binder: $1895-19075$ of 8
Year: 1904

Slogan: A Christmas Morning Kodak

Subject(s): mother / child

Text: Where there's a child, there should the Kodak be. As a

means of keeping green the Christmas memories, or as a gift, it's a holiday delight.

Implied Audience: families / Christmas

Product: Kodak

Photo or Illustration Based: illus

Color or Black and White: BW

Publications: women's magazine

Description / Notes: Drawn for Eastman Kodak, courtesy Colliers weekly.

Binder: $1895-19075$ of 8

Year: 1904

Slogan: Kodak

Subject(s): product - camera

Text: Whatever is good in lenses, in shutters and in mechanical detail is found in the Kodak.

Product: Kodak

Photo or Illustration Based: illus

Color or Black and White: BW

Description / Notes: 5-97.00

Binder: $1895-19075$ of 8

Slogan: Kodak Progress

Subject(s): product - camera

Text: Whatever is new and desirable in picture taking is found in the Kodak System - non curling film, daylight, development-

absolute simplicity.

Product: Kodak

Photo or Illustration Based: photo

Color or Black and White: BW

Binder: $1895-19075$ of 8

Year: 1904

Slogan: "Kodakery"

Subject(s): product - camera 
Product: Kodak

Color or Black and White:

Binder: 1895-1907 5 of 8

Year: 1904

Slogan: Kodak Progress

Subject(s): product - camera

Product: Kodak

Description / Notes: Prices: $\$ 5.00$ to $\$ 97.00$

Binder: 1895-1907 5 of 8

Year: 1904

Slogan: The Christmas Problem Solved--Give a Kodak

Subject(s): product - camera

Product: Kodak

Description / Notes: Price: $\$ 5.00$ to $\$ 97.00$

Binder: $1895-19075$ of 8

Slogan: In War and in Peace The Kodak gets the news

Subject(s): soldier

Text: Indispensable to the correspondent

Implied Audience: soldier

Product: Kodak

Photo or Illustration Based: illus

Color or Black and White: BW

Description / Notes: Copyright 1904 Colliers Weekly

(illustration) "in Korea with the Kodak" By Robert L Dunn sent free upon request.

Binder: $1895-19075$ of 8

Slogan: A Holiday Hint: Kodak

Subject(s): Text: Implied Audience: Christmas

Product: Kodak

Photo or Illustration Based: Text: Color or Black and White: BW

Description / Notes: v. small.

Binder: $1895-19075$ of 8

Year: 1904
Slogan: Developing be Machine

Subject(s): product - darkroom

Product: Kodak Developing Machine

Description / Notes: Price: $\$ 2.00$ to $\$ 10.00$

Binder: 1895-1907 5 of 8

Year: 1904

Slogan: There is no Dark Room in the Kodak

Subject(s): product - darkroom

Product: Kodak Developing Machines

Description / Notes: Price: $\$ 2.00$ to $\$ 10.00$

Binder: 1895-1907 5 of 8

Year: 1904

Slogan: The Kodak at the Front

Subject(s): product - darkroom

Product: Kodak Developing Machines

Description / Notes: Article about a Russian Prince developing photos during war.

Binder: $1895-19075$ of 8

Year: 1904

Slogan: The Convenience of the Kodak Developing Machine

Subject(s): product - darkroom

Product: Kodak Developing Machines

Description / Notes: Price: $\$ 2.00$ to $\$ 10.00$

Binder: 1895-1907 5 of 8

Year: 1904

Slogan: The Kodak Enlarging Camera

Subject(s): product - camera

Product: Kodak Enlarging Camera

Color or Black and White:

Binder: $1895-19075$ of 8

Slogan: Kodak

Subject(s): Kodak Girl

Text: All of the darkroom fuss and bother is removed from

photography by the Kodak way of picture making. Better then the 
old way too. Anybody can make good pictures now that the Kodak Developing Machine has abolished the darkroom.

Implied Audience: women

Product: Kodak System

Photo or Illustration Based: photo

Color or Black and White: BW

Binder: $1895-19075$ of 8

Slogan: Kodak developing machine

Subject(s): Kodak Girl

Text: Kodakery Means photography with the bother left out.

Better results too. ... has abolished the dark room from every step

of picture making. Where the beginner once depended on his

untried judgment, he now depends upon his watch and makes

good pictures from the start. It's simple certain by the Kodak

System.

Implied Audience: women / general

Product: Kodak System

Photo or Illustration Based: photo

Color or Black and White: BW

Binder: $1895-19075$ of 8

Slogan: Kodakery

Subject(s): product - camera

Text: Means photography with the bother left out. Better results

too. No Darkroom, few chemicals...

Product: Kodak System

Photo or Illustration Based: Text: Color or Black and White: BW

Binder: $1895-19075$ of 8

Year: 1904

Slogan: The Kodak System Abolishes most of the opportunities for making mistakes. No dark-room, better pictures.

Subject(s): product - film \& paper

Product: Kodak System

Description / Notes: Kodak Developing Machines Price: $\$ 2.00$ to $\$ 10.00$
Binder: $1895-19075$ of 8

Year: 1904

Slogan: The Changes of Time

Subject(s): product - camera

Product: Kodaks

Color or Black and White:

Binder: $1895-19075$ of 8

Year: 1904

Slogan: Take a Kodak with you to the Exposition

Subject(s): product - camera

Product: Kodaks

Description / Notes: Kodaks Price: $\$ 5.00$ to $\$ 97.00$

Binder: $1895-19075$ of 8

Year: 1904

Slogan: Kodak

Subject(s): product - camera

Product: Kodaks

Description / Notes: Kodaks Price: $\$ 5.00$ to $\$ 97.00$

Binder: $1895-19075$ of 8

Year: 1904

Slogan: Vacation Days are Kodak Days

Subject(s): young man, woman

Text: The Kodaker has all the vacation delights that others have -

and has pictures besides. And there's pleasure in the making as well as in the possessing of Kodak Pictures. Every step is simple now. No darkroom at any stage of the work and better results then ever

Implied Audience: travelers

Product: Kodaks

Photo or Illustration Based: illustration

Color or Black and White: BW

Binder: $1895-19075$ of 8

Year: 1904

Slogan: Kodak

Subject(s): product - camera 
Product: Kodaks and Brownies

Description / Notes: Kodaks Price: $\$ 5.00$ to $\$ 75.00$ Brownies

Price: $\$ 1.00$ to $\$ 2.00$

Binder: $1895-19075$ of 8

Year: 1904

Slogan: Eastman's Kodoid Plates

Subject(s): product - film \& paper

Product: Kodoid Plates

Color or Black and White:

Binder: $1895-19075$ of 8

Year: 1904

Slogan: One Dozen Eastman Koloid Plates

Subject(s): product - film \& paper

Product: Koloid Plates

Description / Notes: Price Per Dozen: \$0.45, \$0.65, \$1.10

Binder: $1895-19075$ of 8

Year: 1904

Slogan: Koloid Plates and N.C. Film

Subject(s): product - film \& paper

Product: Koloid Plates and N.C. Film

Color or Black and White:

Binder: $1895-19075$ of 8

Year: 1904

Slogan: Mezzo-Tone

Subject(s): product - film \& paper

Product: Mezzo-Tone paper

Description / Notes: Price per dozen: \$0.15-\$0.35 Ad also has a

small insert for the Kodak Automatic Shutter.

Binder: $1895-19075$ of 8

Year: 1904

Slogan: Richness in Tone and Texture

Subject(s): product - film \& paper

Product: Mezzo-Tone Paper

Color or Black and White:
Binder: $1895-19075$ of 8

Year: 1904

Slogan: No Curl to Eastman's NC Film

Subject(s): product - film \& paper

Product: NC Film.

Description / Notes: Ad mentions cash prizes for Kodak pictures.

Binder: $1895-19075$ of 8

Slogan: A Holiday Hint: The No 0 Folding Pocket Kodak 6.00

Subject(s): product - camera

Text: The watch in your pocket is no doubt as accurate as the

family clock - likely more accurate. The No 0 folding pocket Kodak

is as accurate as cameras of ten times its bulk. It's for the pocket,

almost for the vest pocket, yet loads for 6 or 12 pictures...

Product: No. 0 Folding pocket Kodak

Color or Black and White: BW

Binder: $1895-19075$ of 8

Year: 1904

Slogan: A Holiday Hint: The No. 0 Folding Pocket Kodak

Subject(s): product - camera

Product: No. 0 Folding Pocket Kodak

Description / Notes: Price: $\$ 6.00$

Binder: $1895-19075$ of 8

Year: 1904

Slogan: The No. 2 Brownie Camera

Subject(s): product - camera

Product: No. 2 Brownie

Description / Notes: Price: $\$ 2.00$

Binder: $1895-19075$ of 8

Year: 1904

Slogan: The New No. 2 Folding Brownie

Subject(s): product - camera

Product: No. 2 Folding Brownie

Description / Notes: Price: $\$ 5.00$ 
Binder: $1895-19075$ of 8

Year: 1904

Slogan: Increased Possibilities. All the Old Simplicity.

Subject(s): product - camera

Product: No. 3 Folding Pocket Kodak

Description / Notes: Price: $\$ 17.50$

Binder: $1895-19075$ of 8

Year: 1904

Slogan: The Highest Achievement in Pocket Photography

Subject(s): product - camera

Product: No. 3 Folding Pocket Kodak

Color or Black and White:

Binder: $1895-19075$ of 8

Year: 1904

Slogan: A New Folding Pocket Kodak

Subject(s): product - camera

Product: No. 3A Folding Pocket Kodak

Description / Notes: Price: $\$ 20.00$

Binder: $1895-19075$ of 8

Year: 1904

Slogan: Eastman's Non-Curling Film

Subject(s): product - film \& paper

Product: Non-Curling Film

Color or Black and White:

Binder: $1895-19075$ of 8

Year: 1904

Slogan: Eastman's NC Film

Subject(s): product - film \& paper

Product: Non-Curling Film

Color or Black and White:

Binder: 1895-1907 5 of 8

Year: 1904

Slogan: Eastman NC Film Lies Flat, Dries Flat

Subject(s): product - film \& paper
Product: Non-Curling Film

Description / Notes: Ad mentions cash prizes for Kodak pictures.

Binder: $1895-19075$ of 8

Year: 1904

Slogan: Eastman's Permanent Bromide

Subject(s): product - film \& paper

Product: Permanent Bromide

Color or Black and White:

Binder: $1895-19075$ of 8

Year: 1904

Slogan: Photography for the Amateur

Subject(s): product - camera

Product: PoCo, Buck-Eye, and American Cameras

Description / Notes: Price: $\$ 1.60$ to $\$ 4.80$

Binder: $1895-19075$ of 8

Year: 1904

Slogan: An Appreciation of Eastman's Sepia Paper

Subject(s): product - film \& paper

Product: Sepia Paper

Description / Notes: Sepia Paper

Binder: $1895-19075$ of 8

Year: 1904

Slogan: Of the many prize-winning pictures in the various CAMERA competitions, nearly $40 \%$ have been made upon

Eastman's Sepia Paper.

Subject(s): product - film \& paper

Product: Sepia Paper

Description / Notes: Quote from "The Camera" August, 1904

Binder: $1895-19075$ of 8

Year: 1904

Slogan: Sepia Post Cards

Subject(s): product - film \& paper

Product: Sepia Post Cards

Description / Notes: Price, two dozen: \$0.25 
Binder: $1895-19075$ of 8

Year: 1904

Slogan: Solio Stands Alone in Richness of Tone and Detail

Subject(s): product - film \& paper

Product: Solio

Color or Black and White:

Binder: $1895-19075$ of 8

Year: 1904

Slogan: For a Dozen years, Solio has stood alone in richness of tone and detail

Subject(s): product - film \& paper

Product: Solio

Color or Black and White:

Binder: $1895-19075$ of 8

Year: 1904

Slogan: The Flexo Kodak

Subject(s): product - camera

Product: The Flexo Kodak

Description / Notes: Price: $\$ 5.00$

Binder: $1895-19075$ of 8

Year: 1904

Slogan: The Flexo Kodak

Subject(s): product - camera

Product: The Flexo Kodak

Description / Notes: Price: $\$ 5.0012$ exposure film cartridge,

$\$ 0.606$ exposure film cartridge: $\$ 0.30$

Binder: 1895-1907 5 of 8

Year: 1904

Slogan: In War and Peace The Kodak gets the news

Subject(s): Soldier

Text: Indispensable to the Correspondent

Implied Audience: Soldiers

Product: The Kodak

Photo or Illustration Based: Illustration

\section{Color or Black and White: BW}

Description / Notes: Caption reads: "Copyright 1904, Colliers Weekly" Famous image of a soldier with a Kodak, standing with hands on his hips.

Binder: $1895-19075$ of 8

Year: 1904

Slogan: An Eastman for a Dollar

Subject(s): product - camera

Product: The No. 1 Brownie Camera

Description / Notes: Price: $\$ 1.00$

Binder: $1895-19075$ of 8

Year: 1904

Slogan: Velox

Subject(s): product - film \& paper

Product: Velox

Color or Black and White:

Binder: $1895-19075$ of 8

Year: 1904

Slogan: Velvet Velox

Subject(s): product - film \& paper

Product: Velox

Color or Black and White:

Binder: $1895-19075$ of 8

Year: 1904

Slogan: Velox Liquid Developer

Subject(s): product - darkroom

Product: Velox Liquid Developer

Color or Black and White:

Binder: $1895-19075$ of 8

Year: 1904

Slogan: They Work Best Together

Subject(s): product - film \& paper

Product: Velox paper and liquid developer

Color or Black and White: 
Binder: $1895-19075$ of 8

Year: 1904

Slogan: Velox Postcards

Subject(s): product - film \& paper

Product: Velox Postcards

Description / Notes: Price per dozen: 20 cents

Binder: $1895-19075$ of 8

Year: 1904

Slogan: Eastman's WD Platinum Paper

Subject(s): product - film \& paper

Product: WD Platinum Paper

Color or Black and White:

Binder: $1895-19075$ of 8

Year: 1904

Slogan: Use Kloro

Subject(s): product - film \& paper

Product: Color or Black and White:

Binder: $1895-19075$ of 8

Slogan: Subject(s): Product: Color or Black and White:

Binder: 1895-1907 6 of 8

Year: 1905

Slogan: Book of the $£ 1,000$ Kodak Exhibition

Subject(s): product - misc.

Product: Book

Description / Notes: Price: $\$ 0.25$ "Reproductions of over 60 pictures from the recent London Competition"

Binder: 1895-1907 6 of 8

Year: 1904

Slogan: Eastman's Royal Bromide Paper

Subject(s): product - film \& paper

Product: Bromide Paper

Color or Black and White:
Binder: 1895-1907 6 of 8

Year: 1905

Slogan: Make Pictures of School friends: The Folding Brownie

Subject(s): camera

Text: It works like a Kodak. Daylight loading, daylight

development. Locks into focus automatically-shutter with iris

diaphragm stops

Implied Audience: parents/children

Product: Brownie

Photo or Illustration Based: illus

Color or Black and White: BW

Publications: American Boy

Description / Notes: Price 5.00 / 9.00

Binder: 1895-1907 6 of 8

Year: $\mathrm{c} 1905$

Slogan: The New Folding Brownies

Subject(s): product - camera

Text: Made on the Kodak System which means: No dark-rooms,

simplicity, success for the beginner.

Product: Brownie

Photo or Illustration Based: Illus

Color or Black and White: BW

Description / Notes: No 2 and No 3 Brownies.

Binder: $1895-19076$ of 8

Year: 1905

Slogan: Decorate your Den

Subject(s): product - camera

Text: With vacation pictures. They are easily made with a Brownie

$\$ 1 \$ 2 \$ 5 \$ 9$

Product: Brownie

Photo or Illustration Based: illus

Color or Black and White: BW

Description / Notes: "Almost a Kodak"

Binder: 1895-1907 6 of 8

Year: $\mathrm{c} 1905$

Slogan: The Dollar Brownie 
Subject(s): product - camera

Text: With all the great facilities ...

Product: brownie

Photo or Illustration Based: illustration

Color or Black and White: BW

Description / Notes: No 1 brownie Possibly out of order in the binder?

Binder: $1895-19076$ of 8

Slogan: Brownie Cameras

Subject(s): product - camera

Text: Made by Kodak Workmen in the Kodak factory. Like Kodak they load and unload in daylight and the films can be developed in daylight. No Darkroom is necessary and the merest beginner can do every step of the work from pressing the button to finishing the prints.

Product: brownie

Photo or Illustration Based: illus

Color or Black and White: BW

Description / Notes: No. 2 Brownie 2.00

Binder: $1895-19076$ of 8

Year: c1905

Slogan: Kodak Home Portraiture

Subject(s): product - camera

Text: By either daylight of flashlight is simple and interesting.

Winter indeed is full of delights for the Kodaker. Portrait studies, evening groups and snowscapes all have their fascinations. The

successful methods are clearly explained in our Kodak books.

Product: cameras

Photo or Illustration Based: Text: Color or Black and White: BW

Binder: $1895-19076$ of 8

Year: c1905

Slogan: The New Daylight Method Kodak Tank Developer

Subject(s): product - darkroom

Text: No Crank Turning During Development. No Dark-Room.

Three years of successful use have proven that the Kodak
Developing machines produce better negatives then were produced by the old darkroom method. The Kodak TANK is an improvement on the machine.

Product: developing tank

Photo or Illustration Based: illus

Color or Black and White: BW

Binder: $1895-19076$ of 8

Year: 1905

Slogan: The New Standard Orthochromatic

Subject(s): product - film \& paper

Product: Dry Plates

Color or Black and White

Binder: $1895-19076$ of 8

Year: 1905

Slogan: Orthonon

Subject(s): product - film \& paper

Product: Dry Plates

Color or Black and White:

Binder: $1895-19076$ of 8

Year: 1904

Slogan: Eastman Flash Sheets

Subject(s): product - camera

Product: Eastman Flash Sheet

Color or Black and White:

Binder: $1895-19076$ of 8

Year: c1905

Slogan: Folding Pocket Kodaks for Christmas

Subject(s): product - camera

Text: Made in a Factory where accuracy is everything.

Product: Folding Pocket Kodak

Color or Black and White: BW

Publications: American Illustrated

Description / Notes: 17.50 / 20.00 No. 3 and 3a

Binder: $1895-19076$ of 8 
Year: c1905

Slogan: Christmas Cameras

Subject(s): product - camera

Text: New Model no. 1 and 1 a folding pocket Kodaks. They work with a snappy precision that is a mechanical delight...

Product: Folding Pocket Kodak

Color or Black and White: BW

Publications: Ainslee's

Description / Notes: 10.00 - 12.00

Binder: $1895-19076$ of 8

Year: c1905

Slogan: New Model 1 and 1a Folding Pocket Kodaks

Subject(s): product - camera

Text: They work with a Snappy Precision that is a Mechanical Delight. With a single motion the front pulls out to the correct focus. In closing the bed automatically covers he working parts. Equipped with out quiet Jarless pocket automatic shutter having instantaneous time and bulb action and iris diaphragm stops.

Product: Folding Pocket Kodak

Photo or Illustration Based: illus

Color or Black and White: BW

Description / Notes: $10.00-12.00$

Binder: $1895-19076$ of 8

Year: 1905

Slogan: The 1905 Hawkeye Cameras

Subject(s): product - camera

Product: Hawk-Eye cameras

Description / Notes: Price: $\$ 5.00$ to $\$ 97.30$

Binder: $1895-19076$ of 8

Year: 1905

Slogan: The Richness of Kloro

Subject(s): product - film \& paper

Product: Kloro

Color or Black and White:

Binder: $1895-19076$ of 8
Year: 1905

Slogan: Landscape Photography with a Kodak

Subject(s): product - camera

Product: Kodak

Description / Notes: article

Binder: $1895-19076$ of 8

Year: 1905

Slogan: Kodak Home Portraiture

Subject(s): product - camera

Product: Kodak

Color or Black and White:

Binder: $1895-19076$ of 8

Year: 1905

Slogan: Our 20 Years of Film Experience

Subject(s): product - film \& paper

Product: Kodak film

Description / Notes: article

Binder: $1895-19076$ of 8

Year: 1905

Slogan: Kodak film for Kodaks

Subject(s): product - film \& paper

Product: Kodak film

Color or Black and White:

Binder: $1895-19076$ of 8

Year: 1905

Slogan: "Kodak Film"

Subject(s): product - film \& paper

Product: Kodak Film

Color or Black and White

Binder: $1895-19076$ of 8

Year: 1905

Slogan: \$2000.00 in Prizes for Kodak Pictures.

Subject(s): product - misc.

Product: Kodak photo contest 


\section{Description / Notes: Contest Closes November 1st}

Binder: $1895-19076$ of 8

Year: c1905

Slogan: There's a World of Delight in Kodak Home Portraiture Subject(s): product - camera

Text: It is simpler than most people think. Beautiful results are obtained by the light of an ordinary window, or by flashlight. A

portrait attachment, at fifty cents adapts the small Kodak to the making of good sized bust photographs. ...

Product: Kodak portrait attachment

Photo or Illustration Based: photo

Color or Black and White: bw

Binder: $1895-19076$ of 8

Year: 1904

Slogan: Most of the Opportunities for making mistakes are

removed by the Kodak System

Subject(s): product - darkroom

Product: Kodak System

Description / Notes: Kodaks Price: $\$ 5.00$ to $\$ 97.00$

Binder: $1895-19076$ of 8

Year: 1904

Slogan: Film at Panama

Subject(s): product - film \& paper

Product: Kodak System

Description / Notes: Letter about shooting film in Panama.

Binder: $1895-19076$ of 8

Year: 1905

Slogan: Again the Tropics

Subject(s): product - film \& paper

Product: Kodak System

Description / Notes: Article

Binder: $1895-19076$ of 8

Year: 1905

Slogan: Tank Development
Subject(s): product - film \& paper

Product: Kodak System

Description / Notes: article

Binder: $1895-19076$ of 8

Year: $1904-5$

Slogan: Bring your vacation home in a Kodak

Subject(s): women taking photos of Dutch people.

Text: Add to the after delights of your holiday with pictures of the people, the places and the sports you are interested in. Every step easy by the Kodak System. Kodaks, \$5.00 to 108.00

Implied Audience: women

Product: Kodak System

Photo or Illustration Based: illus

Color or Black and White: BW

Description / Notes: Illustrated by Edward Penfield. Kodaks, 5108.00 \$

Binder: $1895-19076$ of 8

Year: 1905

Slogan: The Kodak Tank Developer

Subject(s): product - darkroom

Product: Kodak Tank Developer

Color or Black and White:

Binder: $1895-19076$ of 8

Year: 1905

Slogan: A New Kodak Tank Developer

Subject(s): product - film \& paper

Product: Kodak Tank Developer

Description / Notes: Price: $\$ 2.50, \$ 4.00 \$ 5.00$

Binder: $1895-19076$ of 8

Year: 1905

Slogan: The New Daylight Method

Subject(s): product - film \& paper

Product: Kodak Tank Developer

Color or Black and White: 
Binder: $1895-19076$ of 8

Year: 1905

Slogan: Kodak Simplicity

Subject(s): Kodak Girl

Text: has removed most of the opportunities for making mistakes.

No dark-room, few chemicals, little bother. ... The Kodak Girl in

Fair Japan

Implied Audience: women

Product: Kodaks

Photo or Illustration Based: illus

Color or Black and White: BW

Description / Notes: Drawn for Kodak by C. Allen Gilbert

Binder: $1895-19076$ of 8

Year: c1905

Slogan: There are no Game Laws for Those Who Hunt With a Kodak

Subject(s): Men (hunters)

Text: The rod or the gun may be left out, but no nature lover

omits a Kodak from his camp outfit.

Implied Audience: outdoorsmen

Product: Kodaks

Photo or Illustration Based: illus

Color or Black and White: BW

Description / Notes: Drawn for Kodak by A.B. Frost.

Binder: $1895-19076$ of 8

Year: 1904

Slogan: Mezzo-Tone is full of the life and warmth of Summer

Sunshine. A simple self-toning paper. New in surface and effect.

Subject(s): product - film \& paper

Product: Mezzo-Tone paper

Color or Black and White:

Binder: $1895-19076$ of 8

Year: 1904

Slogan: Eastman's Mezzo-Tone

Subject(s): product - film \& paper

Product: Mezzo-Tone paper

\section{Color or Black and White:}

Binder: $1895-19076$ of 8

Year: 1905

Slogan: No Toning Solution needed with Eastman's Mezzo-Tone

Subject(s): product - film \& paper

Product: Mezzo-Tone paper

Color or Black and White:

Binder: $1895-19076$ of 8

Year: 1904

Slogan: Too Fast for Iowa

Subject(s): product - film \& paper

Product: N.C. Film

Description / Notes: Letter from a man who captured a horse race with Kodak film.

Binder: $1895-19076$ of 8

Year: 1904

Slogan: Nepera Solution

Subject(s): product - film \& paper

Product: Nepera Solution

Description / Notes: Price: $\$ 0.25$

Binder: $1895-19076$ of 8

Year: 1905

Slogan: No. 2 Folding Brownie

Subject(s): product - camera

Product: No. 2 Folding Brownie

Description / Notes: Price: $\$ 5.00$

Binder: $1895-19076$ of 8

Year: 1905

Slogan: The New Folding Brownies

Subject(s): product - camera

Product: No. 2 Folding Brownie. No. 3 Folding Brownie

Description / Notes: No. 2 Folding Brownie price: \$5.00. No. 3

Folding Brownie price: $\$ 9.00$ 
Binder: $1895-19076$ of 8

Year: c1905

Slogan: For Vacation Pictures No. 3 Folding Film Premo

Subject(s): product - camera

Text: A compact camera that loads in daylight with the premo film pack. Bausch and Lomb Automatic Shutter. Rising front. Rack and Pinion Focusing.

Product: No. 3 Folding Film Premo

Photo or Illustration Based: Illus

Color or Black and White: BW

Description / Notes: Illegible text on the ad. (photo)

Binder: 1895-1907 6 of 8

Year: 1905

Slogan: No. 3 Folding Film Premo

Subject(s): product - camera

Product: No. 3 Folding Film Premo

Description / Notes: Price: \$18.00.

Binder: $1895-19076$ of 8

Year: 1905

Slogan: Film Convenience--Optical Superiority No. 3 Folding Film Premo

Subject(s): product - camera

Product: No. 3 Folding Film Premo

Description / Notes: Price: $\$ 18.00$.

Binder: $1895-19076$ of 8

Year: 1905

Slogan: Folding Hawk-Eyes

Subject(s): product - camera

Product: No. 3 Folding Hawk-Eye Model 4. No. 4 Folding HawkEye Model 3.

Description / Notes: No. 3 Folding Hawk-Eye Model 4 price: $\$ 22.00$. No. 4 Folding Hawk-Eye Model 3 price: $\$ 27.50$.

Binder: $1895-19076$ of 8

Year: 1904

Slogan: Almost a Kodak
Subject(s): product - camera

Text: The New No.2 Folding Brownie More of a camera than has ever before been offered at the price. Good enough to satisfy

grown people--simple enough for the children. A Christmas delight to either.

Product: No2. Folding Brownie

Photo or Illustration Based: Illus

Color or Black and White: BW

Description / Notes: cost - 5.00

Binder: $1895-19076$ of 8

Year: c1904

Slogan: No. 2 Folding Brownie

Subject(s): product - camera

Text: Built on the Kodak Plan - daylight loading. Daylight

Development. Uses non-curling 6 exposure film cartridges for

$2 / 1 / 4$ by $31 / 4$ pictures.

Product: No2. Folding Brownie

Photo or Illustration Based: illus

Color or Black and White: BW

Description / Notes: cost 5.00 - similar to other brownie ad photo taken of each.

Binder: $1895-19076$ of 8

Year: c1904

Slogan: No. 2 Folding Brownie

Subject(s): product - camera

Text: A wonderfully capable and accurate camera built on the

Kodak plan. Good enough to satisfy experienced photographers, yet so simple that children can use it. ... Loads in daylight with film cartridges

Product: No2. Folding Brownie

Photo or Illustration Based: Illus

Color or Black and White: BW

Description / Notes: cost 5.00 - mentions that it's usable for both children and experienced photographers. Similar to other two brownie ads.

Binder: $1895-19076$ of 8 
Year: 1905

Slogan: Premo Reflecting Camera

Subject(s): product - camera

Product: Premo

Description / Notes: Price: $\$ 85.00$ (without lens)

Binder: $1895-19076$ of 8

Year: 1905

Slogan: Tone your Royal Bromide Enlargements Sepia

Subject(s): product - film \& paper

Product: Royal Re-Developer

Description / Notes: Price per package: $\$ 0.75$

Binder: $1895-19076$ of 8

Year: 1905

Slogan: "Most as Good"

Subject(s): product - film \& paper

Product: Seed Dry Plates

Color or Black and White:

Binder: $1895-19076$ of 8

Year: 1905

Slogan: Solio is Always the Same

Subject(s): product - film \& paper

Product: Solio

Color or Black and White:

Binder: $1895-19076$ of 8

Year: 1905

Slogan: Where Detail is important--Solio

Subject(s): product - film \& paper

Product: Solio

Color or Black and White:

Binder: $1895-19076$ of 8

Year: 1905

Slogan: Proof of the Uniformity of Solio

Subject(s): product - film \& paper

Product: Solio

\section{Color or Black and White}

Binder: $1895-19076$ of 8

Year: 1905

Slogan: The Kodak Enlarging Camera

Subject(s): product - camera

Product: The Kodak Enlarging Camera

Description / Notes: Price: \$15.00. Article.

Binder: $1895-19076$ of 8

Year: c1904

Slogan: The Latest Development in the growth of the Kodak Idea

Subject(s): product - camera

Text: The Screen focus Kodak an instrument which combines all

the advantages of the Kodak Film System with ground glass

focusing. Instantly convertible into a compact plate camera

Product: The Screen Focus Kodak

Photo or Illustration Based: ilus

Color or Black and White: BW

Description / Notes: cost 30.00 converts film camera to plate.

Binder: $1895-19076$ of 8

Year: 1905

Slogan: The Kodak Idea

Subject(s): product - camera

Product: The Screen Focus Kodak

Description / Notes: Price: $\$ 30.00$

Binder: $1895-19076$ of 8

Year: 1905

Slogan: All the Kodak Advantage

Subject(s): product - camera

Product: The Screen Focus Kodak

Description / Notes: Price: $\$ 30.00$

Binder: $1895-19076$ of 8

Year: 1904

Slogan: The Velox Developing Machine

Subject(s): product - film \& paper 
Product: Velox Developing Machine

Description / Notes: Price: $\$ 5.00$

Binder: 1895-1907 6 of 8

Year: c1905

Slogan: Vacation pictures on Velox Post Cards will delight your friends.

Subject(s): Man smoking pipe, printing paper.

Text: You can print them anywhere-no darkroom needed. Mailed

with 1c stamp. Furnished in Regular Velvet, Special Velvet, Special

Portrait, Special Rough velox surfaces.

Implied Audience: men / general

Product: Velox Paper

Photo or Illustration Based: illus

Color or Black and White: BW

Description / Notes: Nepera Division.

Binder: $1895-19076$ of 8

Year: 1904

Slogan: Sepia Tones on Velox and Bromide Paper

Subject(s): product - film \& paper

Product: Velox Re-Developer

Description / Notes: Price: $\$ 0.50$

Binder: $1895-19076$ of 8

Year: 1905

Slogan: Eastman's Velvet Bromide

Subject(s): product - film \& paper

Product: Velvet Bromide

Description / Notes: Article.

Binder: 1895-1907 6 of 8

Year: 1905

Slogan: Hot water is the developer for WD Platinum

Subject(s): product - film \& paper

Product: WD platinum paper

Color or Black and White:

Binder: $1895-19077$ of 8
Year: 1906

Slogan: The 3B Quick Focus Kodak

Subject(s): product - camera

Product: 3B Quick Focus Kodak

Description / Notes: Price $\$ 12.00$

Binder: $1895-19077$ of 8

Year: 1906

Slogan: A New Kodak

Subject(s): product - camera

Product: 4A Folding Kodak

Description / Notes: Price: $\$ 35.00$

Binder: $1895-19077$ of 8

Year: 1906

Slogan: the 4a Folding Kodak

Subject(s): product - camera

Product: 4A Folding Kodak

Description / Notes: Price: $\$ 35.00$

Binder: $1895-19077$ of 8

Year: 1906

Slogan: The New $41 / 4 \times 6$ 1/2 Kodak

Subject(s): product - camera

Product: 4A Folding Kodak

Description / Notes: Price: $\$ 35.00$

Binder: $1895-19077$ of 8

Year: 1906

Slogan: For Detail Use Albuma

Subject(s): product - film \& paper

Product: Albuma paper

Color or Black and White:

Binder: $1895-19077$ of 8

Year: 1906

Slogan: Albuma Preserves Detail

Subject(s): product - film \& paper

Product: Albuma paper 


\section{Color or Black and White:}

Binder: 1895-1907 7 of 8

Year: 1906

Slogan: Now Ready: Angelo Black and White Platinum

Subject(s): product - film \& paper

Product: Angelo Black and White Platinum

Color or Black and White:

Binder: $1895-19077$ of 8

Year: 1906

Slogan: Angelo Sepia Platinum

Subject(s): product - film \& paper

Product: Angelo Sepia Platinum

Color or Black and White:

Binder: $1895-19077$ of 8

Slogan: Brownie Camera

Subject(s): animals / children

Text: There is vacation fun for every boy in a Brownie Camera You

can do every bit of the work from pressing the button to finishing

the print-all without a dark-room. It's inexpensive too...

Implied Audience: parents/children

Product: brownie

Photo or Illustration Based: photo

Color or Black and White: bw

Description / Notes: three photographs, presumably taken by a child with a brownie.

Binder: $1895-19077$ of 8

Year: 1906

Slogan: \$2 Brownie Cameras

Subject(s): product - camera

Product: Brownie Cameras

Description / Notes: Price: $\$ 2.00$

Binder: $1895-19077$ of 8

Year: 1906

Slogan: "They Work Like Kodaks." Brownie Cameras.
Subject(s): product - camera

Product: Brownie Cameras

Description / Notes: No. 2 Brownie Price: $\$ 2.00$ No. 2 Folding Brownie Price: $\$ 5.00$ No. 3 Folding Brownie Price: $\$ 9.00$

Binder: $1895-19077$ of 8

Year: 1906

Slogan: A Dainty Remembrance Eastman Ferro-Prussiate Post

Card

Subject(s): product - film \& paper

Product: Eastman Ferro-Prussiate Post Card

Color or Black and White:

Binder: $1895-19077$ of 8

Year: 1906

Slogan: Broad Sketchy Effects best secured with Eastman's Sepia

Subject(s): product - film \& paper

Product: Eastman's Sepia

Color or Black and White:

Binder: $1895-19077$ of 8

Year: 1906

Slogan: The New No. 3 Folding Brownie

Subject(s): product - camera

Product: Folding Brownie

Description / Notes: Price: $\$ 9.00$

Binder: $1895-19077$ of 8

Year: 1906

Slogan: Hawk-Eyes

Subject(s): product - camera

Product: Hawk-Eye camera

Description / Notes: Prices: $\$ 5.00$ to $\$ 95.00$

Binder: $1895-19077$ of 8

Year: 1906

Slogan: Rich in Silver That's why Kloro is a favorite with the commercial photographer

Subject(s): product - film \& paper 
Product: Kloro paper

Color or Black and White:

Binder: $1895-19077$ of 8

Year: 1906

Slogan: Kloro for Commercial Work

Subject(s): product - film \& paper

Product: Kloro paper

Color or Black and White:

Binder: $1895-19077$ of 8

Slogan: Kodak and Brownie Cameras

Subject(s): boy with dog

Text: made photography so simple that anybody can take good

pictures. Every step is easy: no dark-room is needed and there is as great pleasure in taking and making pictures as there is in

presenting them to friends or preserving them in ones collection.

Implied Audience: parents/children

Product: Kodak

Photo or Illustration Based: illus

Color or Black and White: bw

Description / Notes: small ad. Simple illustration of a boy

photographing a dog atop the KODAK text.

Binder: $1895-19077$ of 8

Slogan: There are no Game Laws for Those Who Hunt With a

Kodak

Subject(s): Caribou swimming in a lake

Implied Audience: hunters / outdoorsman

Product: Kodak

Photo or Illustration Based: photo

Color or Black and White: bw

Description / Notes: Caption: From Pocket Kodak Negative /

Caribou swimming in a Newfoundland Lake.

Binder: $1895-19077$ of 8

Slogan: And in the Sunset Days - Kodak

Subject(s): Elderly couple

Text: It's "Daylight all the way" by the Kodak System. Loading, unloading, developing, printing- all without a dark-room

Implied Audience: the elderly

Product: Kodak

Photo or Illustration Based: illus

Color or Black and White: bw

Description / Notes: Illustration of a man with a Kodak on a

pedestal, photographing his wife reading a book.

Binder: $1895-19077$ of 8

Slogan: Kodak Home Portraiture

Subject(s): Father, Mother, boy posing for a photo taken by a

girl.

Text: It is simpler and easier than most people think. If you use a

Kodak or Brownie camera.

Implied Audience: families

Product: Kodak

Photo or Illustration Based: illus

Color or Black and White: bw

Description / Notes: small ad. Simple drawing of family with girl taking portrait.

Binder: $1895-19077$ of 8

Slogan: The Kodak,

Subject(s): Kodak Girl

Text: It's daylight all the way by the Kodak system, Loading

unloading, developing, printing - all without a darkroom. The

Kodak tank developer, and Velox paper have made the process of finishing the pictures as simple as pressing the button.

Implied Audience: women

Product: Kodak

Photo or Illustration Based: illus

Color or Black and White: bw

Description / Notes: Caption "A Kodak Morning" illustration of a woman walking in a field with Kodak slung on her shoulder.

Binder: $1895-19077$ of 8

Slogan: Told by the Kodak

Subject(s): Kodak Girl

Text: The story of a summer vacation as told by the Kodak. It's 
always an interesting story and it's an easy story to record. For the Kodak works at the bidding of the merest novice. There is no darkroom for any part of Kodak work, it's all simple. Press the button do the rest, or leave it to another - just as you please. The Kodak catalogue tells the details.

Implied Audience: women

Product: Kodak

Photo or Illustration Based: illus

Color or Black and White: bw

Description / Notes: Illustration of a woman looking at images sitting at a window.

Binder: $1895-19077$ of 8

Slogan: The Kodak Story

Subject(s): Kodak Girl

Text: Of summer days grows in charm as the months go by - it's always interesting - it's personal - it tells you of the places, the people and the incidents from your point of view - just as you saw them. And it's an easy story to record, for the Kodak works as the bidding of the merest novice. There is no dark-room for any part of Kodak work, it's all simple. Press the button - do the rest, or leave it to another - just as you please. The Kodak catalogue tells the details.

Implied Audience: women

Product: Kodak

Photo or Illustration Based: illus

Color or Black and White: bw

Description / Notes: Illustration of a woman photographing a

man repairing a car on the side of the road.

Binder: $1895-19077$ of 8

Slogan: Kodak

Subject(s): small boy

Text: Let the Kodak tell the story of the home days of the

children. Photography is simple now-Kodak has made it so. "Kodak Home Portraiture" is the title of a beautiful little book that shows more than a score of such pictures, made both indoors and out, and gives many valuable hints on how to make them. It may be had free at the dealers of by mail.
Implied Audience: families

Product: Kodak

Photo or Illustration Based: photo

Color or Black and White: bw

Description / Notes: photograph in a frame of a boy reading at a table.

Binder: $1895-19077$ of 8

Slogan: There is fun for all the family with a Kodak or Brownie

Camera

Subject(s): father / child

Text: ...

Implied Audience: families

Product: Kodak / brownie

Photo or Illustration Based: illus

Color or Black and White: bw

Description / Notes: small ad, simple drawing of father holding child in front of a lion's cage so that the child can photograph it.

Binder: $1895-19077$ of 8

Slogan: Kodak

Subject(s): Girl and boy

Text: And then again, when snow and ice hold out-doors- Kodak Turn the lens into the home and picture, for the days to come, its hearthstone harmonies. The album of baby and pictures made by the little folks will be held more precious every year. And the picture making is easy now- the Kodak has made it so. No darkroom, few chemicals, no fuss. It's photography with the bother left out

Implied Audience: parents/children

Product: Kodak / brownie

Photo or Illustration Based: illus

Color or Black and White: bw

Description / Notes: caption "Elizabeth Shipsen Green"

illustration of girl photographing brother and toy lamb.

Binder: $1895-19077$ of 8

Year: 1906

Slogan: For Every Boy or Girl there's a Merry Christmas in the 
Kodak Box

Subject(s): product - camera

Product: Kodak Box

Description / Notes: Price for whole Outfit: \$4.00 Individual prices listed.

Binder: $1895-19077$ of 8

Year: 1906

Slogan: The Kodak Box

Subject(s): product - camera

Product: Kodak Box

Description / Notes: Price for whole Outfit: \$4.00 Individual prices listed.

Binder: $1895-19077$ of 8

Year: 1906

Slogan: Everything for Picture Making in the Kodak Box

Subject(s): product - camera

Product: Kodak Box

Color or Black and White:

Binder: $1895-19077$ of 8

Year: 1906

Slogan: There's everything for picture making in the Kodak Box

Subject(s): product - camera

Product: Kodak Box

Description / Notes: Price: $\$ 4.00$

Binder: $1895-19077$ of 8

Slogan: Kodak Box Outfit

Subject(s): Child with animals

Text: You can take good pictures with a Kodak or Brownie camera. Photography is simple now and there is as great pleasure in taking and making pictures as there is in presenting them to friends or preserving them in ones collection.

Implied Audience: parents/children

Product: Kodak box outfit

Photo or Illustration Based: illus

Color or Black and White: bw
Description / Notes: small ad, simple drawing of a child photographing rabbits and chicks.

Binder: $1895-19077$ of 8

Slogan: Kodak Box Outfit

Subject(s): Mother / child with chickens

Text: You can take good pictures with a Kodak or Brownie camera. Photography is simple now and there is as great pleasure in taking and making pictures as there is in presenting them to friends or preserving them in ones collection.

Implied Audience: families

Product: Kodak box outfit

Photo or Illustration Based: illus

Color or Black and White: bw

Description / Notes: small ad, simple drawing of a mother photographing a boy with two chickens.

Binder: $1895-19077$ of 8

Slogan: Kodak Box Outfit

Subject(s): mother / child with horse.

Text: You can take good pictures with a Kodak or Brownie camera. Photography is simple now and there is as great pleasure in taking and making pictures as there is in presenting them to friends or preserving them in ones collection.

Implied Audience: families

Product: Kodak box outfit

Photo or Illustration Based: illus

Color or Black and White: bw

Description / Notes: small ad, simple drawing of a boy photographing his mother and a horse.

Binder: $1895-19077$ of 8

Year: 1906

Slogan: Kodak Box Outfit No. 2

Subject(s): product - camera

Product: Kodak Box Outfit No. 2

Description / Notes: Price $\$ 4.00$

Binder: $1895-19077$ of 8 
Year: 1906

Slogan: The Kodak Dry Mounting Press

Subject(s): product - film \& paper

Product: Kodak Dry Mounting Press

Description / Notes: $5 \times 7$ price: $\$ 15.0011 \times 14$ price: $\$ 50.00$

Binder: $1895-19077$ of 8

Year: 1906

Slogan: Curling troubles end if you use Kodak Dry Mounting

Tissue

Subject(s): product - film \& paper

Product: Kodak Dry-Mounting Tissue

Color or Black and White:

Binder: $1895-19077$ of 8

Year: 1906

Slogan: Kodak Dry Mounting Tissue

Subject(s): product - film \& paper

Product: Kodak Dry-Mounting Tissue

Color or Black and White:

Binder: $1895-19077$ of 8

Year: 1906

Slogan: "Kodak" Film

Subject(s): product - film \& paper

Product: Kodak film

Color or Black and White:

Binder: $1895-19077$ of 8

Year: 1906

Slogan: The Picture Depends on the film, far more than one lens or on camera..

Subject(s): product - film \& paper

Product: Kodak Films

Color or Black and White:

Binder: $1895-19077$ of 8

Year: 1906

Slogan: A Settled Question
Subject(s): product - film \& paper

Product: Kodak films and paper

Description / Notes: Article. Talks about Steichen, Kodak photo contests.

Binder: $1895-19077$ of 8

Year: 1906

Slogan: Special Mounting / Flash Fun / Doctor Kodak

Subject(s): product - film \& paper

Product: Kodak films and paper, flash.

Description / Notes: article.

Binder: $1895-19077$ of 8

Year: 1906

Slogan: Photo Appendicitis

Subject(s): product - film \& paper

Product: Kodak films and paper.

Description / Notes: article.

Binder: $1895-19077$ of 8

Year: 1906

Slogan: Built like a Pocket Kodak / Development Under Difficulties

/ A Non-Curling Start--and Finish

Subject(s): product - film \& paper

Product: Kodak films and paper.

Description / Notes: Article.

Binder: $1895-19077$ of 8

Year: 1906

Slogan: No Moisture / Worth More / Be Sure

Subject(s): product - film \& paper

Product: Kodak films and paper.

Description / Notes: Article.

Binder: $1895-19077$ of 8

Year: 1906

Slogan: Another Velox Success / When to Stop Development

Subject(s): product - film \& paper

Product: Kodak films and paper. 


\section{Description / Notes: article.}

Binder: $1895-19077$ of 8

Year: 1906

Slogan: Preventing Deception

Subject(s): product - film \& paper

Product: Kodak papers

Description / Notes: Article.

Binder: $1895-19077$ of 8

Year: 1906

Slogan: Six to One

Subject(s): product - film \& paper

Product: Kodak System

Description / Notes: article.

Binder: $1895-19077$ of 8

Slogan: Kodak Tank

Subject(s): Man and woman camping

Text: Development is at your convenience when you use the

Kodak Tank. It's all by daylight, as simple as "pressing the button" and the experts say that it gives better results than the dark-room method.

Implied Audience: outdoors

Product: Kodak tank

Photo or Illustration Based: illus

Color or Black and White: bw

Description / Notes: illustration of a man developing film at a

campsite while a woman watches.

Binder: $1895-19077$ of 8

Year: 1906

Slogan: Kodak Tank Developer

Subject(s): product - darkroom

Product: Kodak Tank Developer

Description / Notes: Price: $\$ 2.50, \$ 5.00, \$ 6.00, \$ 7.50$

Binder: $1895-19077$ of 8

Year: 1906
Slogan: The Kodak Method of Tank Development

Subject(s): product - darkroom

Product: Kodak Tank Developer

Color or Black and White:

Binder: $1895-19077$ of 8

Year: 1906

Slogan: Kodak Tank Development

Subject(s): product - film \& paper

Product: Kodak Tank developer.

Color or Black and White:

Binder: $1895-19077$ of 8

Year: 1906

Slogan: Mezzo-Tone

Subject(s): product - film \& paper

Product: Mezzo-Tone paper

Color or Black and White:

Binder: $1895-19077$ of 8

Year: 1906

Slogan: Nepera Solution

Subject(s): product - film \& paper

Product: Nepera Solution

Color or Black and White

Binder: $1895-19077$ of 8

Year: 1906

Slogan: No. 2 Folding Brownie

Subject(s): product - camera

Product: No. 2 Folding Brownie

Description / Notes: Price: $\$ 5.00$

Binder: $1895-19077$ of 8

Year: 1906

Slogan: A New Brownie

Subject(s): product - camera

Product: no. 2a Brownie

Description / Notes: Price: $\$ 3.00$ 
Binder: $1895-19077$ of 8

Year: 1906

Slogan: A New 4x5 Kodak

Subject(s): product - camera

Product: No. 4 Folding Pocket Kodak

Description / Notes: No. 4 Folding Pocket Kodak price: $\$ 20.00$

Price with Automatic Shutter: $\$ 25.00$

Binder: $1895-19077$ of 8

Year: 1906

Slogan: No. 4A Folding Kodak

Subject(s): product - camera

Product: No. 4a Folding Kodak

Description / Notes: Price: $\$ 35.00$

Binder: $1895-19077$ of 8

Year: 1906

Slogan: Orthonon for Correct Values

Subject(s): product - film \& paper

Product: Orthonon Plates.

Color or Black and White:

Binder: $1895-19077$ of 8

Year: 1906

Slogan: Perfect Balance with Orthonon

Subject(s): product - film \& paper

Product: Orthonon Plates.

Color or Black and White:

Binder: $1895-19077$ of 8

Year: 1906

Slogan: No Juggling Standard Orthonon

Subject(s): product - film \& paper

Product: Orthonon Plates.

Color or Black and White:

Binder: $1895-19077$ of 8

Year: 1906
Slogan: The New No. 3 Folding Brownie

Subject(s): product - camera

Product: Price: $\$ 9.00$

Color or Black and White:

Binder: $1895-19077$ of 8

Year: 1906

Slogan: Sepia Enlargements made on Royal Bromide

Subject(s): product - film \& paper

Product: Royal Bromide

Color or Black and White:

Binder: $1895-19077$ of 8

Year: 1906

Slogan: Royal Velox

Subject(s): product - film \& paper

Product: Royal Velox

Color or Black and White:

Binder: $1895-19077$ of 8

Year: 1906

Slogan: By Right of Quality Seed Leads the World

Subject(s): product - film \& paper

Product: Seed Dry Plates

Color or Black and White:

Binder: 1895-1907 7 of 8

Year: 1906

Slogan: Every Case of Seed Plates is as good as every other case.

No waiting for special emulsions from the factory--that's where

you are money ahead.

Subject(s): product - film \& paper

Product: Seed Dry Plates

Color or Black and White:

Binder: $1895-19077$ of 8

Year: 1906

Slogan: If it's in the Negative

Subject(s): product - film \& paper 
Product: Solio Paper

Color or Black and White:

Binder: $1895-19077$ of 8

Year: 1906

Slogan: The Quick Focus Kodak

Subject(s): product - camera

Product: The Quick Focus Kodak

Description / Notes: Price: $\$ 12.00$

Binder: $1895-19077$ of 8

Year: 1906

Slogan: A good negative is worthy of good paper. A poor negative needs it. There's a Velox for every negative.

Subject(s): product - film \& paper

Product: Velox

Color or Black and White:

Binder: $1895-19077$ of 8

Year: 1906

Slogan: Velox, Not cheaper per gross, but cheaper per year.

Subject(s): product - film \& paper

Product: Velox

Color or Black and White:

Binder: $1895-19077$ of 8

Year: 1906

Slogan: Velox The Spare Moment Paper.

Subject(s): product - film \& paper

Product: Velox

Color or Black and White:

Binder: $1895-19077$ of 8

Year: 1906

Slogan: Other Papers are Tried Velox is Used.

Subject(s): product - film \& paper

Product: Velox

Color or Black and White:
Binder: $1895-19077$ of 8

Year: 1906

Slogan: Velox is Recommended for the Novice By the Expert

Subject(s): product - film \& paper

Product: Velox

Color or Black and White:

Binder: $1895-19077$ of 8

Year: 1906

Slogan: A Successful imitation of a Velox Print can only be made

on Velox Paper. There is no Satisfactory substitute

Subject(s): product - film \& paper

Product: Velox

Color or Black and White:

Binder: $1895-19077$ of 8

Year: 1906

Slogan: No Danger from Unfixed Prints with NA Velox Liquid

Developer

Subject(s): product - film \& paper

Product: Velox Liquid Developer

Color or Black and White:

Binder: $1895-19077$ of 8

Year: 1906

Slogan: Velox Re-Developer

Subject(s): product - film \& paper

Product: Velox Re-Developer

Color or Black and White:

Binder: $1895-19078$ of 8

Year: 1907

Slogan: The Top Notch in Pocket Photography

Subject(s): product - camera

Product: 3A Folding Pocket Kodak

Description / Notes: Price: $\$ 20.00$

Binder: $1895-19078$ of 8

Year: 1907 
Slogan: The No. 2A Brownie

Subject(s): product - camera

Product: Brownie

Description / Notes: Price: $\$ 3.00$ Caption reads: "Made with a 2A Brownie--actual size."

Binder: $1895-19078$ of 8

Year: 1907

Slogan: The No. 2 Folding Pocket Brownie

Subject(s): product - camera

Product: Folding Pocket Brownie

Description / Notes: Price: $\$ 5.00$

Binder: $1895-19078$ of 8

Year: 1907

Slogan: Anybody can Kodak. Folding Pocket Brownie

Subject(s): product - camera

Product: Folding Pocket Brownie

Description / Notes: Camera price: \$5.00 Developing Box:

$\$ 1.00$

Binder: $1895-19078$ of 8

Year: 1907

Slogan: Vacation and a Folding Pocket Kodak

Subject(s): product - camera

Product: Folding Pocket Kodak

Color or Black and White:

Binder: $1895-19078$ of 8

Year: 1907

Slogan: Kodak Solves the Christmas Problem

Subject(s): product - camera

Product: Folding Pocket Kodaks

Description / Notes: Price List: No. 1: \$10.00. No. 1A: \$12.00.

No. 1 A Special: $\$ 15.00$ No. 3: $\$ 17.50$ No. 3A: $\$ 20.00$ No. 4 : $\$ 20.00$

Binder: $1895-19078$ of 8

Year: 1907
Slogan: Just an Easy Handful of Kodak Film

Subject(s): product - film \& paper

Product: Kodak film

Description / Notes: Kodaks price: $\$ 5.00$ to $\$ 100.00$

Binder: $1895-19078$ of 8

Year: 1907

Slogan: Highest in America

Subject(s): product - film \& paper

Product: Kodak film

Description / Notes: Ad about the highest smokestack in America.

Binder: $1895-19078$ of 8

Year: 1907

Slogan: It's the Film that's Important

Subject(s): product - film \& paper

Product: Kodak Film

Color or Black and White:

Binder: $1895-19078$ of 8

Year: 1907

Slogan: Time and Temperature

Subject(s): product - darkroom

Product: Kodak Tank Developer

Description / Notes: price: $\$ 2.50$ to $\$ 7.50$

Binder: $1895-19078$ of 8

Year: 1907

Slogan: Trust your films and plates to tank development

Subject(s): product - darkroom

Product: Kodak Tank Developer

Description / Notes: Kodak film tanks: $\$ 2.50$ - \$7.50 Brownie

Development Boxes price: $\$ 1.00$ Premo film pack tank price"

$\$ 3.50$ and $\$ 4.00$. Eastman Plate Tanks: $\$ 3.50$ and $\$ 4.50$

Binder: $1895-19078$ of 8

Year: 1907

Slogan: Put a Darkroom in your suitcase 
Subject(s): product - darkroom Product: Kodak Tank Developer

Color or Black and White:

Binder: $1895-19078$ of 8

Year: 1907

Slogan: The Kodak

Subject(s): product - darkroom

Product: Kodak Tank Developer

Color or Black and White:

Binder: $1895-19078$ of 8

Year: 1907

Slogan: none

Subject(s): Comics

Product: Kodaks

Description / Notes: Two comics about Kodak Cameras from newspapers.

Binder: $1895-19078$ of 8

Year: 1907

Slogan: The No. 1A Folding Pocket Kodak Special

Subject(s): product - camera

Product: No. 1A folding pocket Kodak Special

Description / Notes: Price: \$15.00

Binder: $1895-19078$ of 8

Year: 1907

Slogan: The No. 3 Brownie

Subject(s): product - camera

Product: No. 3 Brownie

Description / Notes: Price: $\$ 4.00$

Binder: $1895-19078$ of 8

Year: 1907

Slogan: The New No. 3 Bulls-Eye Kodak

Subject(s): product - camera

Product: No. 3 Bulls-Eye Kodak

Description / Notes: Price: $\$ 8.00$
Binder: $1895-19078$ of 8

Year: 1907

Slogan: The No. 3A Folding Pocket Kodak

Subject(s): product - camera

Product: No. 3A Folding Pocket Kodak

Description / Notes: Price: $\$ 20.00$

Binder: $1895-19078$ of 8

Year: 1907

Slogan: The Speed Kodak

Subject(s): product - camera

Product: No. 4A Speed Kodak

Description / Notes: No. 4A Speed Kodak with Shutter without lens Price: $\$ 50.00$ with No. 6B lens $\$ 109.50$

Binder: $1895-19078$ of 8

Year: 1907

Slogan: Speed Kodak

Subject(s): product - camera

Product: No. 4A Speed Kodak

Description / Notes: No. 4A Speed Kodak with Shutter without lens Price: $\$ 50.00$ with No. $6 \mathrm{~B}$ lens $\$ 109.50$

Binder: $1895-19078$ of 8

Year: 1907

Slogan: The No. 1 Brownie

Subject(s): product - camera

Product: No.1 Brownie

Description / Notes: Price: $\$ 1.00$

Binder: $1895-19078$ of 8

Year: 1907

Slogan: There's no Better Fun than picture taking

Subject(s): product - camera

Product: The Brownie family

Description / Notes: Price List: No. 1 Brownie: $\$ 1.00$ No. 2

Brownie: $\$ 2.00$ No. 2 A Brownie: $\$ 3.00$ No. 3 Brownie: $\$ 4.00$ No. 2 Folding Pocket Brownie: $\$ 5.00$ No. 3 Folding Pocket Brownie: 
$\$ 9.00$

Binder: $1895-19078$ of 8

Year: 1907

Slogan: A Pocket Brownie

Subject(s): product - camera

Product: The New No. 2 Folding

Description / Notes: Price: $\$ 5.00$

Binder: $1895-19078$ of 8

Year: 1907

Slogan: To Get the full value from your vacation negatives print

them, or have them printed, on Velox

Subject(s): product - film \& paper

Product: Velox

Color or Black and White:

Binder: $1895-19098$ of 8

Year: 1907

Slogan: No. 2a Brownie

Subject(s): bears in a zoo

Text: ...is so simple that anybody can make good pictures from the start.

Implied Audience: Genera

Product: Brownie

Photo or Illustration Based: photo

Color or Black and White: bw

Description / Notes: caption to photo of bears "Made with a No.

2a Brownie - actual size"

Binder: $1895-19098$ of 8

Year: 1906

Slogan: There are no Game Laws for Those Who Hunt With a

Kodak

Subject(s): Deer in the woods

Implied Audience: hunters / outdoorsman

Product: Kodak

Photo or Illustration Based: photo

Color or Black and White: bw
Description / Notes: Caption "Copyright 1906, Sumner W. Matteson. Kodak Negative"

Binder: $1895-19098$ of 8

Slogan: Hunt with a Kodak

Subject(s): Deer in the woods

Text: There are No game laws for those who hunt with a Kodak.

Implied Audience: outdoors

Product: Kodak

Photo or Illustration Based: photo

Color or Black and White: bw

Description / Notes: "from a Kodak negative"

Binder: $1895-19098$ of 8

Slogan: They all remembered the Kodak

Subject(s): Five people at a train station

Text: A vacation without a Kodak is a vacation wasted. A Kodak doubles the value of every journey and adds to the pleasure,

present and future, of every outing. Take a Kodak with you

Implied Audience: travelers

Product: Kodak

Photo or Illustration Based: photo

Color or Black and White: bw

Description / Notes: Photograph of two women, three men waiting at a train station, surrounded by luggage. The woman in the centre holds the Kodak.

Binder: $1895-19098$ of 8

Slogan: Vacation Kodak

Subject(s): hands with camera

Text: Vacation and a folding pocket Kodak All the features that help make the pictures good, with no fussy complications to keep the vacation from being a lazy restful one. Catalogue at the dealers of by mail.

Implied Audience: travelers

Product: Kodak

Photo or Illustration Based: photo?

Color or Black and White: BW 
Binder: $1895-19098$ of 8

Year: 1907

Slogan: Kodak

Subject(s): Kodak Girl

Text: Kodak fits every phase of out-door sport. Its very simplicity, its freedom from intricate detail adapts it to use in comfort in even the coldest weather for it can be easily operated with the hands warmly gloved. And winter is full of outdoor opportunity for picture taking, while the long evenings give the time and inclination for the delights of developing and of printing. Kodak means

photography with the bother left out.

Implied Audience: out-doors / women

Product: Kodak

Photo or Illustration Based: photo

Color or Black and White: bw

Description / Notes: woman in muff, carrying Kodak in case over her shoulder.

Binder: $1895-19098$ of 8

Year: 1907

Slogan: Kodak

Subject(s): Kodak Gir

Text: There's twice the pleasure in the journey and twice the pleasure afterward if you Kodak. And anybody can make good pictures. It's simple from start to finish by the Kodak system.

Press the button-do the rest-or leave it to another-just as you please. Kodak means photography with the bother left out.

Implied Audience: travelers

Product: Kodak

Photo or Illustration Based: photo-illustration

Color or Black and White: bw

Description / Notes: Woman holding a camera, and a muff against a drawn background of the white house (?)

Binder: $1895-19098$ of 8

Slogan: Kodak

Subject(s): Kodak Girl

Text: There's twice the pleasure in the journey and twice the pleasure afterward if you Kodak. And anybody can make good pictures. It's simple from start to finish by the Kodak system. Press the button-do the rest-or leave it to another-just as you please. Kodak means photography with the bother left out.

Implied Audience: women

Product: Kodak

Photo or Illustration Based: Illus

Color or Black and White: bw

Description / Notes: woman loading film while resting foot on suitcase.

Binder: $1895-19098$ of 8

Slogan: There's More to Vacation when you Kodak

Subject(s): Kodak Girl

Text: More pleasure at the moment and afterward the added

charm of pictures that tell the vacation story. And it is all so simple by the Kodak system that the merest novice can make good

pictures from the start. Kodak has removed most opportunities for making mistakes.

Implied Audience: women

Product: Kodak

Photo or Illustration Based: photo

Color or Black and White: bw

Publications: Seaway (?)

Description / Notes: woman in a had holding a Kodak.

Binder: $1895-19098$ of 8

Slogan: "The World is mine - I own a Kodak"

Subject(s): Kodak Girl

Text: Take a Kodak with you, and picture from your own

viewpoint, not merely the places that interest you but also the

companions who help make your trip enjoyable. Anybody can take good pictures with a Kodak.

Implied Audience: women / travelers

Product: Kodak

Photo or Illustration Based: photo

Color or Black and White: BW

Description / Notes: Photograph of a woman with her Kodak, as a Porter helps her onto a train. 
Binder: $1895-19098$ of 8

Slogan: There are no Game Laws for Those Who Hunt With a Kodak

Subject(s): Man with camera, heavy backpack

Implied Audience: hunters / outdoorsman

Product: Kodak

Photo or Illustration Based: photo

Color or Black and White: BW

Description / Notes: Photo of a man with a pipe, large camping pack on his back on one knee taking a photograph.

Binder: $1895-19098$ of 8

Year: 1907

Slogan: The Kodak Christmas Story

Subject(s): mother / child

Text: Wherever children are there's a Christmas story, yes, an all the year round story of the Kodak record--a story that grows in interest as the years go by. Let the grown folks with a Kodak and the children with a Brownie join in building the family Kodak Book. And there's no better way to begin than with pictures of Christmas day.

Implied Audience: families

Product: Kodak

Photo or Illustration Based: illustration

Color or Black and White: bw

Description / Notes: woman sitting in a chair reading to a young child.

Binder: $1895-19098$ of 8

Slogan: A Kodak Story

Subject(s): mother / child

Text: Of the baby- a serial story from the teddy bear days,

through childhood and school days until home ties are broken -

such a story has the charm of human interest that endures. And

it's an easy story to record, for the Kodak works are the bidding of the merest novice. There is no dark-room for any part of the

Kodak work, it's all simple. Press the button-do the rest-or leave it

to another-just as you please

Implied Audience: families
Product: Kodak

Photo or Illustration Based: illustration

Color or Black and White: bw

Description / Notes: Woman and baby sitting outdoors, the baby is playing with a teddy bear.

Binder: $1895-19098$ of 8

Slogan: The Baby's picture

Subject(s): mother / child

Text: It makes no difference how often baby goes to the photographer and for the sake of admiring relatives his visits should be frequent. The record of his infant days is incomplete unless there are home pictures to supplement the more formal studio photographs. Mother or father or sister can readily make a series of pictures of the little one that will grow more and more precious year by year. Picture taking is easy now and inexpensive too, the Kodak has made it so.

Implied Audience: families

Product: Kodak

Photo or Illustration Based: photo

Color or Black and White: bw

Description / Notes: woman on knees taking a photograph of a baby with a Kodak.

Binder: $1895-19098$ of 8

Slogan: Let the Children Kodak

Subject(s): Young girl, kitten

Text: And then, in turn Kodak the children.

Implied Audience: families

Product: Kodak

Photo or Illustration Based: photo

Color or Black and White: BW

Description / Notes: Photo of a small girl sitting on a porch

photographing a kitten.

Binder: $1895-19098$ of 8

Year: 1907

Slogan: Take a Kodak with You

Subject(s): young man, woman 
Text: Twill double the pleasure of every outing and give twice the pleasure afterward. And anybody can make good pictures by the Kodak system. No dark-room - no bother. Every step is simple.

Implied Audience: travelers

Product: Kodak

Photo or Illustration Based: photo-illustration

Color or Black and White: bw

Description / Notes: woman sitting on a rock taking a shot, man

leaning over her, pointing at something off camera. Painting of

horses in the background.

Binder: $1895-19098$ of 8

Slogan: Let the Children Kodak

Subject(s): Young boy and gir

Text: And then, in turn, Kodak the children. There's pleasure and instruction,- there's education in taking pictures, there's a

constantly growing charm in the pictures themselves. And by the Kodak system picture taking is perfectly simple, whether one merely presses the button and lets another do the rest or

whether the delights of picture taking be added to the delights of picture making.

Implied Audience: parents.

Product: Kodak / brownie

Photo or Illustration Based: photo

Color or Black and White: bw

Description / Notes: Photo of a boy photographing a girl.

Binder: $1895-19098$ of 8

Year: 1907

Slogan: Just an Easy Handful of Kodak Film

Subject(s): hand w film.

Text: Will tell the story of your summer vacation. There's film for a dozen pictures in each cartridge, the weight is trifling. The Kodak itself slips into the pocket and the picture making is simple from start to finish. Press the button-do the rest-or leave it to anotherjust as you please

Implied Audience: travelers

Product: Kodak film

Photo or Illustration Based: photo?
Color or Black and White: bw

Description / Notes: Jun-07

Binder: $1895-19098$ of 8

Year: 1907

Slogan: "Highest in America"

Subject(s): product - misc

Text: The manufacture of film to the Kodak standard requires

perfect basic materials... The film you use is more important then

the camera you use. Look for "Kodak" on the spool.

Product: Kodak film

Photo or Illustration Based: photo

Color or Black and White: bw

Description / Notes: long text, not all copied.

Binder: $1895-19098$ of 8

Year: 1907

Slogan: "The Kodak on the Farm"

Subject(s): Chicken and chicks

Text: A beautifully illustrated little book containing a score of

pictures that show how interesting the Kodak may be made in the country.

Implied Audience: farmers

Product: Kodak System

Photo or Illustration Based: photo

Color or Black and White: bw

Description / Notes: smaller version of above ad, less text.

Binder: $1895-19098$ of 8

Year: 1907

Slogan: "The Kodak on the Farm"

Subject(s): Horse, vignette.

Text: Is the title of a beautifully illustrated little book that has just come from the press. It contains a score of pictures that show how interesting the Kodak may be made in the country and it explains clearly the simplicity of the Kodak system of photograph

Implied Audience: farmers

Product: Kodak System

Photo or Illustration Based: photo 


\section{Color or Black and White: bw}

Description / Notes: same as above ad, but with different

photograph, smaller ad.

Binder: $1895-19098$ of 8

Year: 1908

Slogan: "The Kodak on the Farm"

Subject(s): horses at fence

Text: Is the title of a beautifully illustrated little book that has just come from the press. It contains a score of pictures that show how interesting the Kodak may be made in the country and it explains clearly the simplicity of the Kodak system of photography...

Implied Audience: farmers

Product: Kodak system

Photo or Illustration Based: photo

Color or Black and White: bw

Description / Notes: not all text copied.

Binder: $1895-19098$ of 8

Year: 1907

Slogan: Kodak means photography with the bother left out.

Subject(s): Man reading an album by the fireplace

Text: The vacation story-as told by his Kodak. In every home

there's a story for the Kodak to record-not merely a travel story and the story of the summer holiday, but the story of Christmas, of the winter evening gatherings and of the house party. But above all is the serial story of the children, from the days of wild gallops across the nursery floor... And it's all a simple story to record, for the Kodak system of photography has removed most of the opportunities for mistakes. It's inexpensive now and there's no darkroom for any part of the work.

Implied Audience: men / general

Product: Kodak System

Photo or Illustration Based: photo

Color or Black and White: bw

Description / Notes: not all text copied

Binder: $1895-19098$ of 8

Slogan: Kodak Tells the Story
Subject(s): navy

Text: Of every world event- whether it is a peaceful cruise of a great Navy around the globe, a war that changes the map or continents, a devastating earthquake, or the inauguration of a president, you now have the photographic story quickly and

accurately told in the illustrated press. ...

Implied Audience: Soldiers

Product: Kodak System

Photo or Illustration Based: photo

Color or Black and White: bw

Description / Notes: Photograph of four navy men on the deck of a ship, two photographing, two developing.

Binder: $1895-19098$ of 8

Year: $\mathrm{c} 1907$

Slogan: "The Kodak on the Farm"

Subject(s): young girl with a doll

Text: A beautifully illustrated little book containing a score of pictures that show how interesting the Kodak may be made in the country.

Implied Audience: farmers

Product: Kodak System

Photo or Illustration Based: photo

Color or Black and White: bw

Description / Notes: photograph of a young girl holding a doll

standing in the corner of a wallpapered room.

Binder: $1895-19098$ of 8

Year: 1907

Slogan: There's no Dark room with a Kodak Tank

Subject(s): Kodak girls

Text: Every step is simple and easy but more than that, it means better pictures. The success of the tank development idea has now been absolutely proven by the fact that many leading professional photographers, although they have every dark room convenience, use our tank system of development for all their work...

Experience is in the Tank.

Implied Audience: women

Product: Kodak tank 
Photo or Illustration Based: photo

Color or Black and White: bw

Description / Notes: two women, one with arms around the other looking at a roll of negatives.

Binder: $1895-19098$ of 8

Year: 1907

Slogan: Kodak Tank

Subject(s): man outdoors developing film

Text: Film Development with the Kodak Tank not only means an end to dark-room bother but it means better results than can be obtained in the old way. The time and temperature are the only

factors to watch. The Experience is in the Tank

Implied Audience: outdoors

Product: Kodak tank

Photo or Illustration Based: photo

Color or Black and White: bw

Description / Notes: Man smoking pipe, reading a paper while developing film outdoors.

Binder: $1895-19098$ of 8

Year: 1908

Slogan: Put a Dark Room your Suit Case

Subject(s): product - darkroom

Text: Don't lose half the vacation pleasure by waiting until you

return home before developing your films. Get results on the spot, and then if you like, sent postcards from your own negatives to

your friends. Anybody can develop perfectly, ...

Product: Kodak tank

Photo or Illustration Based: illustration

Color or Black and White: bw

Binder: $1895-19098$ of 8

Year: 1906

Slogan: There's no Dark Room with a Kodak Tank

Subject(s): three boys developing film

Text: Every step is simple and easy but more than that, it means better pictures. The success of the tank development idea has now been absolutely proven by the fact that many leading professional photographers, although they have every dark room convenience, use our tank system of development for all their work...

Experience is in the Tank.

Implied Audience: parents/children

Product: Kodak Tank

Photo or Illustration Based: photo

Color or Black and White: bw

Description / Notes: Photo of three boys at a table developing film.

Binder: $1895-19098$ of 8

Slogan: "The Kodak Baby Book"

Subject(s): toddler

Text: A helpful little booklet telling how to successfully keep a

photographic record of the baby-how to make the pictures, how to arrange them. Illustrated with a dozen home pictures of the authors own baby.

Implied Audience: families

Product: the Kodak baby book

Photo or Illustration Based: photo

Color or Black and White: bw

Description / Notes: photo of child reading a book

Binder: 1909-1913 1 of 6

Slogan: Eastman Plate Tank

Subject(s): product - darkroom

Text: You can spend more of your time outside the door when you use the Eastman Plate Tank. Less trouble, More comfort, better results.

Product: Eastman plate tank

Photo or Illustration Based: photo

Color or Black and White: bw

Description / Notes: Photo of darkroom door (maybe not relevant)

Binder: 1909-1913 Ad Books 1 of 6

Slogan: Eastman Plate Tank

Subject(s): two women

Text: Where the tank enters, the darkroom worries end. Not 
merely less trouble but better negatives for those who use the Eastman Plate Tank

Implied Audience: women / general

Product: Eastman plate tank

Photo or Illustration Based: photo

Color or Black and White: bw

Description / Notes: photograph of two women outside a

darkroom with plates. (maybe not relevant?)

Binder: 1909-1913 Ad Books 1 of 6

Year: 1909

Slogan: First Gun From the 1908 Conventions

Subject(s): product - film \& paper

Product: Angelo paper

Description / Notes: Talks about the contests won on Angelo paper

Binder: 1909-1913 Ad Books 1 of 6

Year: 1909

Slogan: Second Gun from the Conventions

Subject(s): product - film \& paper

Product: Angelo paper

Description / Notes: Talks about Illinois Convention May 5-7 1908

Binder: 1909-1913 Ad Books 1 of 6

Year: 1909

Slogan: Angelo Has Won Four Grand Prizes

Subject(s): product - film \& paper

Product: Angelo paper

Description / Notes: Talks about the contests won on Angelo paper

Binder: 1909-1913 Ad Books 1 of 6

Year: 1909

Slogan: Again Angelo

Subject(s): product - film \& paper

Product: Angelo paper

Description / Notes: Talks about the contests won on Angelo paper

Binder: 1909-1913 Ad Books 1 of 6

Year: 1909

Slogan: And Now in the East

Subject(s): product - film \& paper

Product: Angelo paper

Description / Notes: Talks about the contests won on Angelo

paper -- New England Convention July 28-30

Binder: 1909-1913 Ad Books 1 of 6

Year: 1909

Slogan: Still the Pace Maker Angelo

Subject(s): product - film \& paper

Product: Angelo paper

Color or Black and White:

Binder: 1909-1913 Ad Books 1 of 6

Year: 1909

Slogan: Angelo

Subject(s): product - film \& paper

Product: Angelo Platinum Paper

Color or Black and White:

Binder: 1909-1913 Ad Books 1 of 6

Year: 1909

Slogan: First in Every Class at Boston

Subject(s): product - film \& paper

Product: Angelo Platinum Paper

Description / Notes: Talks about photo contest results at the New England Convention, August 27-29th

Binder: 1909-1913 Ad Books 1 of 6

Year: 1909

Slogan: Get more money for your work--Raise the Quality with Angelo Sepia and you can raise the price.

Subject(s): product - film \& paper

Product: Angelo Sepia Paper

Color or Black and White: 
Binder: 1909-1913 Ad Books 1 of 6 Year: 1909

Slogan: Angelo Sepia

Subject(s): product - film \& paper

Product: Angelo Sepia Paper

Color or Black and White:

Binder: 1909-1913 Ad Books 1 of 6

Year: 1909

Slogan: Carbon effects in Sepia without carbon difficulties

Subject(s): product - film \& paper

Product: Aristo Gold Paper

Color or Black and White:

Binder: 1909-1913 Ad Books 1 of 6

Year: 1909

Slogan: Aristo

Subject(s): product - film \& paper

Product: Aristo paper

Color or Black and White:

Binder: 1909-1913 Ad Books 1 of 6

Year: 1909

Slogan: Aristo Platino

Subject(s): product - film \& paper

Product: Aristo paper

Color or Black and White:

Binder: 1909-1913 Ad Books 1 of 6 Year: 1909

Slogan: Print, Wash, Fix. Aristo Gold Post Cards

Subject(s): product - film \& paper

Product: Aristo paper

Color or Black and White:

Binder: 1909-1913 Ad Books 1 of 6

Year: 1909

Slogan: Aristo Gold Paper
Subject(s): product - film \& paper

Product: Aristo paper

Color or Black and White:

Binder: 1909-1913 Ad Books 1 of 6

Year: 1909

Slogan: For nineteen years thousands of show cases have been demonstrating the permanent brilliancy of Aristo

Subject(s): product - film \& paper

Product: Aristo paper

Color or Black and White:

Binder: 1909-1913 Ad Books 1 of 6

Year: 1909

Slogan: No Toning--Aristo Gold Paper

Subject(s): product - film \& paper

Product: Aristo paper

Color or Black and White:

Binder: 1909-1913 Ad Books 1 of 6

Year: 1909

Slogan: For the Average Negative for the Average Customer--

Aristo has the quality

Subject(s): product - film \& paper

Product: Aristo paper

Color or Black and White:

Binder: 1909-1913 Ad Books 1 of 6

Year: 1909

Slogan: The paper you know all about--Aristo

Subject(s): product - film \& paper

Product: Aristo paper

Color or Black and White

Binder: 1909-1913 Ad Books 1 of 6

Year: 1909

Slogan: For Superior Results--Aristo Gold Post Cards

Subject(s): product - film \& paper

Product: Aristo paper 


\section{Color or Black and White:}

Binder: 1909-1913 Ad Books 1 of 6 Year: 1909

Slogan: Twenty Years of Aristo

Subject(s): product - film \& paper

Product: Aristo paper

Description / Notes: 1889-1909

Binder: 1909-1913 Ad Books 1 of 6 Year: 1909

Slogan: No "Perhaps" With Aristo

Subject(s): product - film \& paper

Product: Aristo paper

Color or Black and White:

Binder: 1909-1913 Ad Books 1 of 6 Year: 1909

Slogan: A short cut to good results--Aristo Gold Paper

Subject(s): product - film \& paper

Product: Aristo paper

Color or Black and White:

Binder: 1909-1913 Ad Books 1 of 6 Year: 1909

Slogan: No Toning with Aristo Gold Post Cards

Subject(s): product - film \& paper

Product: Aristo paper

Color or Black and White:

Binder: 1909-1913 Ad Books 1 of 6

Year: 1909

Slogan: For Sepias rich in detail and gradation use Aristo Gold Paper

Subject(s): product - film \& paper

Product: Aristo paper

Color or Black and White:

Binder: 1909-1913 Ad Books 1 of 6
Year: 1909

Slogan: Not promise, but performance--Aristo

Subject(s): product - film \& paper

Product: Aristo paper

Color or Black and White:

Binder: 1909-1913 Ad Books 1 of 6

Year: 1909

Slogan: Brownie Enlarging Cameras

Subject(s): product - camera

Product: Brownie Enlarging Camera

Description / Notes: No. 2 Brownie Enlarging Camera for $5 \times 7$ enlargements: $\$ 2.00$. No. 3 Brownie Enlarging Camera: $\$ 3.00$. No. 4 Brownie Enlarging Cameras $\$ 4.00$

Binder: 1909-1913 Ad Books 1 of 6

Year: 1909

Slogan: A Folding Pocket Brownie / Doubles / Before You Go

Subject(s): product - camera

Product: Brownie, Kodaks, Velox Paper

Description / Notes: article.

Binder: 1909-1913 Ad Books 1 of 6

Year: 1909

Slogan: The Kodak Dry Mounting Press

Subject(s): product - misc.

Product: Dry Mounting Press

Description / Notes: 5x7 Dry Mounting Press: \$15.00 11x14 press: $\$ 50.00$

Binder: 1909-1913 Ad Books 1 of 6

Year: 1909

Slogan: It's poor economy to spoil good films, good plates, and good papers with cheap chemicals. To be sure they're pure insist on the mark that denotes the Eastman Quality:

Subject(s): product - film \& paper

Product: Eastman chemicals

Color or Black and White: 
Binder: 1909-1913 Ad Books 1 of 6

Year: 1909

Slogan: Flashlight Time

Subject(s): product - misc.

Product: Eastman Flash cartridge

Description / Notes: Prices: Eastman's Spreader flash cartridges No. 1: $\$ 0.60$. No. 2: $\$ 0.40$ No. 3: $\$ 0.25$ Spreader Flash Pistol $\$ 0.50$. Flash Sheets, No.1: \$0.25 No. $2 \$ 0.40$ No. $3 \$ 0.60$

Binder: 1909-1913 Ad Books 1 of 6

Year: 1909

Slogan: You Must Have the Best Chemicals if you want the best

results. There's only one answer:

Subject(s): product - Chemicals

Product: Eastman Kodak Company Tested Chemicals

Color or Black and White:

Binder: 1909-1913 Ad Books 1 of 6

Year: 1909

Slogan: Spending is Saving when you spend money for chemicals

of known quality and strength. To be sure, look for this label:

Subject(s): product - Chemicals

Product: Eastman Kodak Company Tested Chemicals

Color or Black and White:

Binder: 1909-1913 Ad Books 1 of 6

Year: 1909

Slogan: It's the Film that is Important

Subject(s): product - film \& paper

Product: Eastman N.C. Film

Color or Black and White:

Binder: 1909-1913 Ad Books 1 of 6

Year: 1909

Slogan: The Eastman Plate Tank For Results.--

Subject(s): product - darkroom

Product: Eastman Plate Tank

Description / Notes: Price: $\$ 4.50$
Binder: 1909-1913 Ad Books 1 of 6

Year: 1909

Slogan: The Eastman Plate Tank

Subject(s): product - darkroom

Product: Eastman Plate Tank

Description / Notes: Price: \$4.50

Binder: 1909-1913 Ad Books 1 of 6

Year: 1909

Slogan: Let Your Tank do the Work

Subject(s): product - darkroom

Product: Eastman Plate Tank

Description / Notes: Price: \$4.50

Binder: $1909-1913$ Ad Books 1 of 6

Year: 1909

Slogan: 8x10 Now Ready

Subject(s): product - darkroom

Product: Eastman Plate Tank

Description / Notes: price: $\$ 10.00$

Binder: 1909-1913 Ad Books 1 of 6

Year: 1909

Slogan: Results

Subject(s): product - film \& paper

Product: Eastman Plate Tank

Description / Notes: Price: Tank 5x7 \$4.50

Binder: 1909-1913 Ad Books 1 of 6

Year: 1909

Slogan: Just Right / That Vacation List

Subject(s): product - misc.

Product: Eastman thermometer, Kodak Cameras, developing

tanks, flash,

Description / Notes: Article.

Binder: 1909-1913 Ad Books 1 of 6

Year: 1909

Slogan: There is Money in enlargements made on Eastman's 
Permanent Bromide

Subject(s): product - film \& paper

Product: Eastman's Permanent Bromide

Color or Black and White:

Binder: 1909-1913 Ad Books 1 of 6 Year: 1909

Slogan: Eastman's WD Platinum Paper

Subject(s): product - film \& paper

Product: Eastman's Platinum Paper

Color or Black and White:

Binder: 1909-1913 Ad Books 1 of 6

Year: 1909

Slogan: Tested Chemicals

Subject(s): product - film \& paper

Product: Eastman's PS developer.

Color or Black and White:

Binder: 1909-1913 Ad Books 1 of 6

Year: 1909

Slogan: Not at your expense / Simple Enlarging

Subject(s): product - film \& paper

Product: Film and Brownie Enlarging Camera.

Description / Notes: article.

Binder: 1909-1913 Ad Books 1 of 6

Year: 1909

Slogan: Two Ways / A Printing Suggestion

Subject(s): product - camera

Product: Flash

Description / Notes: Article.

Binder: 1909-1913 Ad Books 1 of 6

Year: 1909

Slogan: Flash Light Possibilities.

Subject(s): product - misc.

Product: Flash

Description / Notes: article.
Binder: 1909-1913 Ad Books 1 of 6

Year: 1909

Slogan: Flash Failures / Mounting on Thin Leaves

Subject(s): product - misc.

Product: Flash / Kodak Dry Mounting Tissue

Description / Notes: article.

Binder: 1909-1913 Ad Books 1 of 6

Year: 1909

Slogan: How you may know / Take it with you

Subject(s): product - darkroom

Product: Kodak chemicals, Film tank

Description / Notes: article.

Binder: 1909-1913 Ad Books 1 of 6

Year: 1909

Slogan: That Neat, Professional Finish--Kodak Dry Mounting Tissue

Subject(s): product - film \& paper

Product: Kodak Dry-Mounting Tissue

Color or Black and White:

Binder: 1909-1913 Ad Books 1 of 6 Year: 1909

Slogan: Variety / Emergency Cases

Subject(s): product - film \& paper

Product: Kodak film and papers.

Description / Notes: Article.

Binder: 1909-1913 Ad Books 1 of 6

Year: 1909

Slogan: Not the Same

Subject(s): product - film \& paper

Product: Kodak film and papers.

Description / Notes: Article.

Binder: 1909-1913 Ad Books 1 of 6

Year: 1909 
Slogan: Good Enlargements from Flat Negatives

Subject(s): product - film \& paper

Product: Kodak film and papers.

Description / Notes: Article.

Binder: 1909-1913 Ad Books 1 of 6

Year: 1909

Slogan: Missed Pleasures

Subject(s): product - film \& paper

Product: Kodak film and papers.

Description / Notes: Article.

Binder: 1909-1913 Ad Books 1 of 6 Year: 1909

Slogan: Cleverly Linked / Only One / Portrait Possibilities Subject(s): product - film \& paper

Product: Kodak system

Description / Notes: article.

Binder: 1909-1913 Ad Books 1 of 6 Year: 1909

Slogan: The First Season

Subject(s): product - camera

Product: Kodaks

Description / Notes: Article.

Binder: 1909-1913 Ad Books 1 of 6

Year: 1909

Slogan: Nepera

Subject(s): product - film \& paper

Product: Nepera Paper

Color or Black and White:

Binder: 1909-1913 Ad Books 1 of 6 Year: 1909

Slogan: Velvet Nepera

Subject(s): product - film \& paper

Product: Nepera Paper

Color or Black and White:
Binder: 1909-1913 Ad Books 1 of 6 Year: 1909

Slogan: Re-Developed Royal Nepera

Subject(s): product - film \& paper

Product: Nepera Paper

Color or Black and White:

Binder: 1909-1913 Ad Books 1 of 6

Year: 1909

Slogan: Nepera

Subject(s): product - film \& paper

Product: Nepera Paper

Color or Black and White:

Binder: 1909-1913 Ad Books 1 of 6

Year: 1909

Slogan: Nepera Waxing Solution

Subject(s): product - film \& paper

Product: Nepera Waxing Solution

Color or Black and White:

Binder: 1909-1913 Ad Books 1 of 6

Year: 1909

Slogan: A Pocketful of Quality

Subject(s): product - camera

Product: No.1A Folding Pocket Kodak Special.

Color or Black and White:

Binder: 1909-1913 Ad Books 1 of 6

Year: 1909

Slogan: Now Ready --Ozobrome

Subject(s): product - film \& paper

Product: Ozobrome carbon prints

Color or Black and White:

Binder: 1909-1913 Ad Books 1 of 6

Year: 1909

Slogan: Ozobrome 
Subject(s): product - film \& paper Product: Ozobrome carbon prints

Description / Notes: Article.

Binder: 1909-1913 Ad Books 1 of 6 Year: 1909

Slogan: The "All By Lamp Light Way"

Subject(s): product - film \& paper

Product: Ozobrome carbon prints

Description / Notes: Article.

Binder: 1909-1913 Ad Books 1 of 6

Year: 1909

Slogan: Carbon with the Bother Left Out

Subject(s): product - film \& paper

Product: Ozobrome carbon prints

Description / Notes: article.

Binder: 1909-1913 Ad Books 1 of 6

Year: 1909

Slogan: A Wide Usefulness / Large Pictures

Subject(s): product - camera

Product: Portrait attachment / Kodak cameras

Description / Notes: article.

Binder: 1909-1913 Ad Books 1 of 6

Year: 1909

Slogan: Royal Nepera

Subject(s): product - film \& paper

Product: Royal Nepera

Color or Black and White:

Binder: 1909-1913 Ad Books 1 of 6

Year: 1909

Slogan: The Prints Lie Flat

Subject(s): product - film \& paper

Product: Royal Nepera

Color or Black and White:
Binder: 1909-1913 Ad Books 1 of 6

Year: 1909

Slogan: For Those Vacation Pictures--Royal Velox

Subject(s): product - film \& paper

Product: Royal Velox

Color or Black and White:

Binder: 1909-1913 Ad Books 1 of 6

Year: 1909

Slogan: Seed Tropical

Subject(s): product - film \& paper

Product: Seed Dry Plates

Color or Black and White:

Binder: 1909-1913 Ad Books 1 of 6

Year: 1909

Slogan: The "Been Through the mill" Photographer never questions price--just orders Seed, the cheapest plate when measured by results."

Subject(s): product - film \& paper

Product: Seed Dry Plates

Color or Black and White:

Binder: 1909-1913 Ad Books 1 of 6 Year: 1909

Slogan: Speed and Latitude

Subject(s): product - film \& paper

Product: Seed Dry Plates

Color or Black and White:

Binder: 1909-1913 Ad Books 1 of 6

Year: 1909

Slogan: Be Sure it's Seed

Subject(s): product - film \& paper

Product: Seed Dry Plates

Color or Black and White:

Binder: 1909-1913 Ad Books 1 of 6

Year: 1909 
Slogan: Extract from Seed Demonstrators Instructions Subject(s): product - film \& paper

Product: Seed Dry Plates

Color or Black and White:

Binder: 1909-1913 Ad Books 1 of 6

Year: 1909

Slogan: Made for those who know by those who know

Subject(s): product - film \& paper

Product: Seed Dry Plates

Color or Black and White:

Binder: 1909-1913 Ad Books 1 of 6

Year: 1909

Slogan: Seed

Subject(s): product - film \& paper

Product: Seed Dry Plates

Color or Black and White:

Binder: 1909-1913 Ad Books 1 of 6

Year: 1909

Slogan: The Few Cents More You Pay for Seed Comes Back in Dollars

Subject(s): product - film \& paper

Product: Seed Dry Plates

Color or Black and White:

Binder: 1909-1913 Ad Books 1 of 6

Year: 1909

Slogan: Seed Sown in the studio, yield dollars.

Subject(s): product - film \& paper

Product: Seed Dry Plates

Color or Black and White:

Binder: 1909-1913 Ad Books 1 of 6

Year: 1909

Slogan: Fine and Clear

Subject(s): product - film \& paper

Product: Seed Dry Plates

\section{Color or Black and White:}

Binder: 1909-1913 Ad Books 1 of 6 Year: 1909

Slogan: Standard Polychrome

Subject(s): product - film \& paper

Product: Standard Dry Plate

Color or Black and White:

Binder: 1909-1913 Ad Books 1 of 6

Year: 1909

Slogan: In Winter--Use Standard Polychrome

Subject(s): product - film \& paper

Product: Standard Dry Plate

Color or Black and White:

Binder: 1909-1913 Ad Books 1 of 6

Year: 1909

Slogan: Standard Thermic

Subject(s): product - film \& paper

Product: Standard Dry Plate

Color or Black and White:

Binder: 1909-1913 Ad Books 1 of 6

Year: 1909

Slogan: A Significant Fact / All By Daylight Enlarging

Subject(s): product - film \& paper

Product: Tank Developing / Brownie Enlarging Camera

Description / Notes: Article.

Binder: 1909-1913 Ad Books 1 of 6

Year: 1909

Slogan: Each Year Has Seen Better

Subject(s): product - film \& paper

Product: Velox

Color or Black and White:

Binder: 1909-1913 Ad Books 1 of 6

Year: 1909 
Slogan: The Reason the Majority of Amateurs use Velox is that they are sure of good results. Velox is the anytime paper with a grade for any negative.

Subject(s): product - film \& paper

Product: Velox paper

Color or Black and White:

Binder: 1909-1913 Ad Books 1 of 6

Year: 1909

Slogan: Print those vacation negatives on Velox

Subject(s): product - film \& paper

Product: Velox paper

Color or Black and White:

Binder: 1909-1913 Ad Books 1 of 6

Year: 1909

Slogan: For the Vacation: Velox Post Cards

Subject(s): product - film \& paper

Product: Velox Post Cards

Description / Notes: Price: \$0.20 per Doz.

Binder: 1909-1913 Ad Books 2 of 6

Year: 1909

Slogan: To get the long price, use Angelo

Subject(s): product - film \& paper

Product: Angelo paper

Color or Black and White:

Binder: 1909-1913 Ad Books 2 of 6

Year: 1909

Slogan: None As good as Angelo

Subject(s): product - film \& paper

Product: Angelo paper

Color or Black and White:

Binder: 1909-1913 Ad Books 2 of 6

Year: 1909

Slogan: Angelo has the Quality on which the judges agree Subject(s): product - film \& paper
Product: Angelo paper

Color or Black and White:

Binder: 1909-1913 Ad Books 2 of 6

Year: 1909

Slogan: Novice Simplicity, expert results--Aristo Gold Paper

Subject(s): product - film \& paper

Product: Aristo Gold Paper

Color or Black and White:

Binder: 1909-1913 Ad Books 2 of 6

Year: 1909

Slogan: For Your Best Negatives

Subject(s): product - film \& paper

Product: Aristo Gold Paper

Color or Black and White

Binder: 1909-1913 Ad Books 2 of 6

Year: 1909

Slogan: Permanent Pictures of Quality

Subject(s): product - film \& paper

Product: Aristo Gold Paper

Color or Black and White:

Binder: 1909-1913 Ad Books 2 of 6

Year: 1909

Slogan: For Your Very Best Negatives

Subject(s): product - film \& paper

Product: Aristo Gold Post Cards

Color or Black and White:

Binder: 1909-1913 Ad Books 2 of 6

Year: 1909

Slogan: Carbon printing is difficult, but true carbon effects are easy with Aristo Gold Post Cards.

Subject(s): product - film \& paper

Product: Aristo Gold Post Cards

Color or Black and White 
Binder: 1909-1913 Ad Books 2 of 6

Year: 1909

Slogan: Carbon Sepia or Purple tones easily produced on Aristo Gold post cards

Subject(s): product - film \& paper

Product: Aristo Gold Post Cards

Color or Black and White:

Binder: 1909-1913 Ad Books 2 of 6

Year: 1909

Slogan: You and your customer--both are satisfied when you

deliver Aristo Prints.

Subject(s): product - darkroom

Product: Aristo paper

Color or Black and White:

Binder: 1909-1913 Ad Books 2 of 6

Year: 1909

Slogan: Rich Sepias and purples--semi matte surface

Subject(s): product - film \& paper

Product: Aristo paper

Color or Black and White:

Binder: 1909-1913 Ad Books 2 of 6

Year: 1909

Slogan: The straight road to good results--Aristo

Subject(s): product - film \& paper

Product: Aristo paper

Color or Black and White:

Binder: 1909-1913 Ad Books 2 of 6

Year: 1909

Slogan: A Little slower to work, but a lot quicker to sell--Aristo

has the quality that satisfies both you and your customers

Subject(s): product - film \& paper

Product: Aristo paper

Color or Black and White:

Binder: 1909-1913 Ad Books 2 of 6
Year: 1909

Slogan: You can see your way

Subject(s): product - film \& paper

Product: Aristo paper

Color or Black and White:

Binder: 1909-1913 Ad Books 2 of 6

Year: 1909

Slogan: The Acknowledged Standard--Aristo

Subject(s): product - film \& paper

Product: Aristo paper

Color or Black and White:

Binder: 1909-1913 Ad Books 2 of 6

Year: 1909

Slogan: Back up your operating skill with Aristo

Subject(s): product - film \& paper

Product: Aristo paper

Color or Black and White:

Binder: 1909-1913 Ad Books 2 of 6

Year: 1909

Slogan: Imitations are "Nearly"--Aristo is.

Subject(s): product - film \& paper

Product: Aristo paper

Color or Black and White

Binder: 1909-1913 Ad Books 2 of 6

Year: 1909

Slogan: 21 years of Supremacy

Subject(s): product - film \& paper

Product: Aristo paper

Color or Black and White

Binder: 1909-1913 Ad Books 2 of 6

Year: 1909

Slogan: Artura Iris

Subject(s): product - film \& paper

Product: Artura Iris Pape 


\section{Color or Black and White:}

Binder: 1909-1913 Ad Books 2 of 6 Year: 1909

Slogan: For High-Grade Portraiture

Subject(s): product - film \& paper

Product: Artura Iris Paper

Color or Black and White:

Binder: 1909-1913 Ad Books 2 of 6

Year: 1909

Slogan: Large Pictures

Subject(s): product - camera

Product: Brownie Enlarging Camera

Description / Notes: Price: No.2: $\$ 2.00$ No.3: $\$ 3.00$ No. 4: $\$ 4.00$

Binder: 1909-1913 Ad Books 2 of 6

Year: 1909

Slogan: Works like a printing frame--Brownie Enlarging Camera

Subject(s): product - camera

Product: Brownie Enlarging Camera

Description / Notes: Price: No.2: \$2.00 No.3: \$3.00 No. 4: $\$ 4.00$

Binder: 1909-1913 Ad Books 2 of 6

Year: 1909

Slogan: Bromide Enlarging with a Kodak

Subject(s): product - camera

Product: Brownie Enlarging Camera

Color or Black and White:

Binder: 1909-1913 Ad Books 2 of 6

Year: 1909

Slogan: The Eastman Adjustable Lens Hood

Subject(s): product - camera

Product: Eastman Adjustable Lens Hood

Description / Notes: No. 1 price: $\$ 2.00$ No. 2 Price: $\$ 2.50$
Binder: 1909-1913 Ad Books 2 of 6

Year: 1909

Slogan: There is profit for you in enlargements on Eastman

Bromide Paper.

Subject(s): product - film \& paper

Product: Eastman Bromide Paper

Color or Black and White

Binder: 1909-1913 Ad Books 2 of 6

Year: 1909

Slogan: Enlarged Portraits on Eastman Bromide Paper

Subject(s): product - film \& paper

Product: Eastman Bromide Paper

Color or Black and White:

Binder: 1909-1913 Ad Books 2 of 6

Year: 1909

Slogan: Eastman Spreader Flash Cartridges

Subject(s): product - film \& paper

Product: Eastman Flash Sheets

Description / Notes: Spreader Flash Cartridges price: \$0.25.

Spreader Flash Cartridge Pistol: $\$ 0.50$. No. 1 Flash Sheets: $\$ 0.25$

No. 2 Flash Sheets: $\$ 0.40$. No. 3 Flash Sheets: $\$ 0.60$.

Binder: 1909-1913 Ad Books 2 of 6

Year: 1909

Slogan: A Square yard of illumination with a $4 \times 5$ Eastman Flash Sheet

Subject(s): product - misc.

Product: Eastman Flash Sheets

Description / Notes: No. 1 price: $\$ 0.25$ No. 2 Price: $\$ 0.40$ No 3 price: $\$ 0.60$

Binder: 1909-1913 Ad Books 2 of 6

Year: 1909

Slogan: Flash possibilities / Best for winter pictures.

Subject(s): product - misc.

Product: Eastman Flash Sheets

Description / Notes: article. 
Binder: 1909-1913 Ad Books 2 of 6 Year: 1909

Slogan: Easily Found / By Flashlight

Subject(s): product - misc.

Product: Eastman Flash Sheets, negative album

Description / Notes: article.

Binder: 1909-1913 Ad Books 2 of 6

Year: 1909

Slogan: You just must have the best chemicals if you want the

best results. There's only one answer.

Subject(s): product - Chemicals

Product: Eastman Kodak Company Tested Chemicals

Color or Black and White:

Binder: 1909-1913 Ad Books 2 of 6

Year: 1909

Slogan: Demand a certificate of character from your chemical assistants:

Subject(s): product - Chemicals

Product: Eastman Kodak Company Tested Chemicals

Color or Black and White:

Binder: 1909-1913 Ad Books 2 of 6

Year: 1909

Slogan: Your Protection

Subject(s): product - Chemicals

Product: Eastman Kodak Company Tested Chemicals

Color or Black and White:

Binder: 1909-1913 Ad Books 2 of 6

Year: 1909

Slogan: The Sign of Continued Interest

Subject(s): product - Chemicals

Product: Eastman Kodak Company Tested Chemicals

Color or Black and White:

Binder: 1909-1913 Ad Books 2 of 6
Year: 1909

Slogan: Use Chemicals of Quality if you want results

Subject(s): product - Chemicals

Product: Eastman Kodak Company Tested Chemicals

Color or Black and White:

Binder: 1909-1913 Ad Books 2 of 6

Year: 1909

Slogan: Every atom pure and the best that science can produce

Subject(s): product - Chemicals

Product: Eastman Kodak Company Tested Chemicals

Color or Black and White:

Binder: 1909-1913 Ad Books 2 of 6

Year: 1909

Slogan: The result of years of experience. Eastman's NC Film

Subject(s): product - film \& paper

Product: Eastman N.C. Film

Color or Black and White:

Binder: 1909-1913 Ad Books 2 of 6

Year: 1909

Slogan: There is comfort as well as convenience and results in the Eastman Plate Tank

Subject(s): product - darkroom

Product: Eastman Plate Tank

Color or Black and White

Binder: 1909-1913 Ad Books 2 of 6

Year: 1909

Slogan: Everything to suit you with the Eastman Plate Tank

Subject(s): product - darkroom

Product: Eastman Plate Tank

Description / Notes: 5x7 Tank: \$4.50 8x10 tank: \$10.00

Binder: 1909-1913 Ad Books 2 of 6

Year: 1909

Slogan: Be comfortable.

Subject(s): product - film \& paper 
Product: Eastman Plate Tank

Description / Notes: $4 \times 5$ price: $\$ 3.505 \times 7$ price: $\$ 4.508 \times 10$

price: $\$ 10.00$

Binder: 1909-1913 Ad Books 2 of 6

Year: 1909

Slogan: For Crisp Clean, Clear Negatives, use the Eastman Plate Tank

Subject(s): product - film \& paper

Product: Eastman Plate Tank

Color or Black and White:

Binder: 1909-1913 Ad Books 2 of 6

Year: 1909

Slogan: For the Best Studio in Town--Eastman Platinum

Subject(s): product - film \& paper

Product: Eastman's Platinum Paper

Color or Black and White:

Binder: 1909-1913 Ad Books 2 of 6

Year: 1909

Slogan: The Slightest tinge of warmth in Eastman Platinum

Subject(s): product - film \& paper

Product: Eastman's Platinum Paper

Color or Black and White:

Binder: 1909-1913 Ad Books 2 of 6

Year: 1909

Slogan: Accepted on sight--Eastman Platinum

Subject(s): product - film \& paper

Product: Eastman's Platinum Paper

Color or Black and White:

Binder: 1909-1913 Ad Books 2 of 6

Year: 1909

Slogan: Make your better grade enlargements on Eastman's Royal

Bromide

Subject(s): product - film \& paper

Product: Eastman's Royal Bromide

\section{Color or Black and White}

Binder: 1909-1913 Ad Books 2 of 6

Year: 1909

Slogan: Kodak Enlarging Camera

Subject(s): product - camera

Product: Kodak Enlarging Camera

Color or Black and White:

Binder: 1909-1913 Ad Books 2 of 6

Year: 1909

Slogan: No Darkroom when you use the Kodak Film Tank

Subject(s): product - darkroom

Product: Kodak film tank

Color or Black and White:

Binder: 1909-1913 Ad Books 2 of 6

Year: 1909

Slogan: Zeiss Kodak f6.3

Subject(s): product - camera

Product: Kodak lenses

Description / Notes: Article.

Binder: 1909-1913 Ad Books 2 of 6

Year: 1909

Slogan: The Autotime Scale / Zeiss-Kodak Quality

Subject(s): Product - camera

Product: Kodak lenses

Description / Notes: article.

Binder: 1909-1913 Ad Books 2 of 6

Year: 1909

Slogan: Take a Dark-Room With You

Subject(s): product - darkroom

Product: Kodak Tank Developer

Color or Black and White:

Binder: 1909-1913 Ad Books 2 of 6

Year: 1909 
Slogan: Just Right / The Problem Solver / Anti Handicap

Subject(s): product - misc.

Product: Negative Album, Kodak system

Description / Notes: Article.

Binder: 1909-1913 Ad Books 2 of 6 Year: 1909

Slogan: A New Nepera--Royal Pure White

Subject(s): product - film \& paper

Product: Nepera Paper

Color or Black and White:

Binder: 1909-1913 Ad Books 2 of 6

Year: 1909

Slogan: The New R.O.C. Post Card Camera

Subject(s): product - camera

Product: R.O.C. Post Card Camera

Description / Notes: Price: $\$ 12.00$

Binder: 1909-1913 Ad Books 2 of 6

Year: 1909

Slogan: Highest Price, Largest Sale--that tells the story of Seed

Supremacy

Subject(s): product - film \& paper

Product: Seed Dry Plates

Color or Black and White:

Binder: 1909-1913 Ad Books 2 of 6

Year: 1909

Slogan: When you want a good plate, there are several--when you want the best, there is but one: Seed

Subject(s): product - film \& paper

Product: Seed Dry Plates

Color or Black and White:

Binder: 1909-1913 Ad Books 2 of 6

Year: 1909

Slogan: Lengthen the short days with Seed 27

Subject(s): product - film \& paper
Product: Seed Dry Plates

Color or Black and White:

Binder: 1909-1913 Ad Books 2 of 6

Year: 1909

Slogan: For Crisp Brilliant Slides--Seed Red Label

Subject(s): product - film \& paper

Product: Seed Dry Plates

Color or Black and White:

Binder: 1909-1913 Ad Books 2 of 6

Year: 1909

Slogan: Seed Red Label

Subject(s): product - film \& paper

Product: Seed Dry Plates

Color or Black and White:

Binder: 1909-1913 Ad Books 2 of 6

Year: 1909

Slogan: Your Confidence in Seed Plates is well grounded

Subject(s): product - film \& paper

Product: Seed Dry Plates

Color or Black and White:

Binder: 1909-1913 Ad Books 2 of 6

Year: 1909

Slogan: Seed

Subject(s): product - film \& paper

Product: Seed Dry Plates

Color or Black and White:

Binder: 1909-1913 Ad Books 2 of 6 Year: 1909

Slogan: Seed is dry-plate perfection

Subject(s): product - film \& paper

Product: Seed Dry Plates

Color or Black and White:

Binder: 1909-1913 Ad Books 2 of 6 
Year: 1909

Slogan: Full gradation, with short exposures, when you use a

Standard Polychrome

Subject(s): product - film \& paper

Product: Standard Dry Plate

Color or Black and White:

Binder: 1909-1913 Ad Books 2 of 6

Year: 1909

Slogan: The Plate without Frills Standard Thermic

Subject(s): product - film \& paper

Product: Standard Dry Plate

Color or Black and White:

Binder: 1909-1913 Ad Books 2 of 6

Year: 1909

Slogan: At Vacation's end there's Velox

Subject(s): product - film \& paper

Product: Velox

Color or Black and White:

Binder: 1909-1913 Ad Books 2 of 6

Year: 1909

Slogan: The Velox Book will help you select the right grade of the right paper.

Subject(s): product - film \& paper

Product: Velox paper

Color or Black and White:

Binder: 1909-1913 Ad Books 2 of 6

Year: 1909

Slogan: Select the proper grade and Velox

Subject(s): product - film \& paper

Product: Velox paper

Color or Black and White:

Binder: 1909-1913 Ad Books 2 of 6

Year: 1909

Slogan: For "going away" time--Velox Post Cards
Subject(s): product - film \& paper

Product: Velox Post Cards

Color or Black and White:

Binder: 1909-1913 Ad Books 3 of 6

Year: 1909

Slogan: Angelo Sepia Platinum

Subject(s): product - film \& paper

Product: Angelo paper

Color or Black and White

Binder: 1909-1913 Ad Books 3 of 6

Year: 1909

Slogan: Angelo

Subject(s): product - film \& paper

Product: Angelo paper

Color or Black and White:

Binder: 1909-1913 Ad Books 3 of 6

Year: 1909

Slogan: The tone beauty of Angelo

Subject(s): product - film \& paper

Product: Angelo paper

Color or Black and White:

Binder: 1909-1913 Ad Books 3 of 6

Year: 1909

Slogan: Angelo Wins Both

Subject(s): product - film \& paper

Product: Angelo paper

Color or Black and White:

Binder: 1909-1913 Ad Books 3 of 6

Year: 1909

Slogan: For pleasing sepia tone and beautiful gradation nothing equals the popular platinum paper Angelo

Subject(s): product - film \& paper

Product: Angelo paper

Color or Black and White 
Binder: 1909-1913 Ad Books 3 of 6

Year: 1909

Slogan: The Whole Story of Print Perfection told in a word is:

Aristo

Subject(s): product - film \& paper

Product: Aristo

Color or Black and White:

Binder: 1909-1913 Ad Books 3 of 6

Year: 1909

Slogan: Aristo gold post cards yield prints of carbon quality

Subject(s): product - film \& paper

Product: Aristo Gold Paper

Color or Black and White:

Binder: 1909-1913 Ad Books 3 of 6

Year: 1909

Slogan: Use Aristo Gold Post Cards

Subject(s): product - film \& paper

Product: Aristo Gold Post Cards

Color or Black and White:

Binder: 1909-1913 Ad Books 3 of 6

Year: 1909

Slogan: For Permanent Prints of pleasing tone and texture: Aristo

Subject(s): product - film \& paper

Product: Aristo paper

Color or Black and White:

Binder: 1909-1913 Ad Books 3 of 6

Year: 1909

Slogan: For Post card prints of carbon quality use Aristo Gold Post

Cards

Subject(s): product - film \& paper

Product: Aristo paper

Color or Black and White:

Binder: 1909-1913 Ad Books 3 of 6
Year: 1909

Slogan: Full value is apparent when the prints are on Aristo

Subject(s): product - film \& paper

Product: Aristo paper

Color or Black and White:

Binder: 1909-1913 Ad Books 3 of 6

Year: 1909

Slogan: No toning chemicals required with Aristo Gold Paper

Subject(s): product - film \& paper

Product: Aristo paper

Color or Black and White:

Binder: 1909-1913 Ad Books 3 of 6

Year: 1909

Slogan: A satisfied customer is one whose prints are finished on

Aristo

Subject(s): product - film \& paper

Product: Aristo paper

Color or Black and White:

Binder: 1909-1913 Ad Books 3 of 6

Year: 1909

Slogan: Establish new business and hold established business with Aristo

Subject(s): product - film \& paper

Product: Aristo paper

Color or Black and White:

Binder: 1909-1913 Ad Books 3 of 6

Year: 1909

Slogan: Aristo

Subject(s): product - film \& paper

Product: Aristo paper

Color or Black and White:

Binder: 1909-1913 Ad Books 3 of 6

Year: 1909

Slogan: Artura Iris for Portraiture 
Subject(s): product - film \& paper Product: Artura Iris Paper

Color or Black and White:

Binder: 1909-1913 Ad Books 3 of 6

Year: 1909

Slogan: For sparkle and detail print it on Artura Iris

Subject(s): product - film \& paper

Product: Artura Iris Paper

Color or Black and White:

Binder: 1909-1913 Ad Books 3 of 6

Year: 1909

Slogan: Artura Iris Solves the Problem

Subject(s): product - film \& paper

Product: Artura Iris Paper

Color or Black and White:

Binder: 1909-1913 Ad Books 3 of 6

Year: 1909

Slogan: After all is said and done, "quality counts."

Subject(s): product - film \& paper

Product: Artura Iris Paper

Color or Black and White:

Binder: 1909-1913 Ad Books 3 of 6

Year: 1909

Slogan: With Other Photographers

Subject(s): product - misc.

Product: Booklet

Description / Notes: Price: $\$ 2.50$, India tint paper, booklet by

Ryland W. Phillips

Binder: 1909-1913 Ad Books 3 of 6

Year: 1909

Slogan: Carbon Black Artura

Subject(s): product - film \& paper

Product: Carbon Black Artura

Color or Black and White:
Binder: 1909-1913 Ad Books 3 of 6

Year: 1909

Slogan: Make enlargements and money with Eastman Bromide Paper

Subject(s): product - film \& paper

Product: Eastman Bromide Paper

Color or Black and White:

Binder: 1909-1913 Ad Books 3 of 6

Year: 1909

Slogan: Eastman Bromide Paper

Subject(s): product - film \& paper

Product: Eastman Bromide Paper

Color or Black and White:

Binder: 1909-1913 Ad Books 3 of 6

Year: 1909

Slogan: Enlarge the Beauty of that Kodak Negative on Eastman

Bromide Paper

Subject(s): product - film \& paper

Product: Eastman Bromide Paper

Color or Black and White:

Binder: 1909-1913 Ad Books 3 of 6

Year: 1909

Slogan: Eastman EB Platinum

Subject(s): product - film \& paper

Product: Eastman EB Platinum

Description / Notes: EB = Etching Black

Binder: $1909-1913$ Ad Books 3 of 6

Year: 1909

Slogan: Topmost Prices Eastman EB Platinum

Subject(s): product - film \& paper

Product: Eastman EB Platinum

Color or Black and White:

Binder: 1909-1913 Ad Books 3 of 6 
Year: 1909

Slogan: The rare warm black tone secured with Eastman EB Platinum

Subject(s): product - film \& paper

Product: Eastman EB Platinum

Color or Black and White:

Binder: 1909-1913 Ad Books 3 of 6

Year: 1909

Slogan: Eastman ES Platinum

Subject(s): product - film \& paper

Product: Eastman ES Platinum

Description / Notes: ES = Etching Sepia

Binder: 1909-1913 Ad Books 3 of 6

Year: 1909

Slogan: Eastman ES Platinum

Subject(s): product - film \& paper

Product: Eastman ES Platinum

Color or Black and White:

Binder: 1909-1913 Ad Books 3 of 6

Year: 1909

Slogan: Eastman Ferro-Prussiate Post Cards

Subject(s): product - film \& paper

Product: Eastman Ferro-Prussiate Post Cards

Color or Black and White:

Binder: 1909-1913 Ad Books 3 of 6

Year: 1909

Slogan: Eastman flash sheet holder

Subject(s): product - misc.

Product: Eastman Flash Sheets

Color or Black and White:

Binder: 1909-1913 Ad Books 3 of 6

Year: 1909

Slogan: The Eastman Focusing Curtain

Subject(s): product - camera
Product: Eastman Focusing Curtain

Description / Notes: Price: $\$ 5.00$

Binder: 1909-1913 Ad Books 3 of 6

Year: 1909

Slogan: If you are having trouble don't blame the films, the plates

or the paper. Test your Chemicals, or better and easier still, use

E.K.C. Tested Chemicals

Subject(s): product - Chemicals

Product: Eastman Kodak Company Tested Chemicals

Color or Black and White:

Binder: 1909-1913 Ad Books 3 of 6

Year: 1909

Slogan: Active Chemicals tested by experts bear this mark:

Subject(s): product - Chemicals

Product: Eastman Kodak Company Tested Chemicals

Color or Black and White:

Binder: 1909-1913 Ad Books 3 of 6

Year: 1909

Slogan: Eastman's Permanent Crystal Pyro

Subject(s): product - Chemicals

Product: Eastman Kodak Company Tested Chemicals

Color or Black and White:

Binder: 1909-1913 Ad Books 3 of 6

Year: 1909

Slogan: A new developing agent Enol

Subject(s): product - Chemicals

Product: Eastman Kodak Company Tested Chemicals

Color or Black and White:

Binder: 1909-1913 Ad Books 3 of 6

Year: 1909

Slogan: A perfect developing agent of remarkable quality is Enol

Subject(s): product - Chemicals

Product: Eastman Kodak Company Tested Chemicals

Color or Black and White: 
Binder: 1909-1913 Ad Books 3 of 6

Year: 1909

Slogan: Our interest in your final results is the reason for

Subject(s): product - Chemicals

Product: Eastman Kodak Company Tested Chemicals

Color or Black and White:

Binder: 1909-1913 Ad Books 3 of 6

Year: 1909

Slogan: Eastman Permanent Crystal Pyro

Subject(s): product - Chemicals

Product: Eastman Kodak Company Tested Chemicals

Color or Black and White:

Binder: 1909-1913 Ad Books 3 of 6

Year: 1909

Slogan: The mark on chemical labels indicates reliable chemicals

tested for photographic purposes

Subject(s): product - Chemicals

Product: Eastman Kodak Company Tested Chemicals

Color or Black and White:

Binder: 1909-1913 Ad Books 3 of 6

Year: 1909

Slogan: It is only worth our while to spend the time, and money

that we do in the scientific testing of chemicals, and in our careful putting up of those chemicals, because such work expense means

better results for the users of our sensitized products.

Subject(s): product - Chemicals

Product: Eastman Kodak Company Tested Chemicals

Color or Black and White:

Binder: 1909-1913 Ad Books 3 of 6

Year: 1909

Slogan: Enol

Subject(s): product - Chemicals

Product: Eastman Kodak Company Tested Chemicals

Color or Black and White:
Binder: $1909-1913$ Ad Books 3 of 6

Year: 1909

Slogan: Chemicals of proper strength, carefully tested to preserve the high quality of our sensitized materials, bear this seal:

Subject(s): product - Chemicals

Product: Eastman Kodak Company Tested Chemicals

Color or Black and White:

Binder: 1909-1913 Ad Books 3 of 6

Year: 1909

Slogan: Stays where it belongs. Eastman Permanent Crystal Pyro

Subject(s): product - Chemicals

Product: Eastman Kodak Company Tested Chemicals

Color or Black and White:

Binder: 1909-1913 Ad Books 3 of 6

Year: 1909

Slogan: The Hall-mark of Chemical Quality

Subject(s): product - Chemicals

Product: Eastman Kodak Company Tested Chemicals

Color or Black and White:

Binder: 1909-1913 Ad Books 3 of 6

Year: 1909

Slogan: One of the tested chemicals Enol

Subject(s): product - Chemicals

Product: Eastman Kodak Company Tested Chemicals

Color or Black and White:

Binder: 1909-1913 Ad Books 3 of 6

Year: 1909

Slogan: The difference between tested chemicals and those of

unknown quality is distinguishable by this mark on chemical labels

Subject(s): product - Chemicals

Product: Eastman Kodak Company Tested Chemicals

Color or Black and White:

Binder: 1909-1913 Ad Books 3 of 6 
Year: 1909

Slogan: Eastman Mask Cutter No. 2

Subject(s): product - misc.

Product: Eastman Mask Cutter No. 2

Description / Notes: Price: $\$ 10.00$

Binder: 1909-1913 Ad Books 3 of 6

Year: 1909

Slogan: Made for the Kodak by Kodak experts. Eastman NC Film

Subject(s): product - film \& paper

Product: Eastman N.C. Film

Color or Black and White:

Binder: 1909-1913 Ad Books 3 of 6

Year: 1909

Slogan: Eastman NC Film

Subject(s): product - film \& paper

Product: Eastman N.C. Film

Color or Black and White:

Binder: 1909-1913 Ad Books 3 of 6

Year: 1909

Slogan: Your Pictures

Subject(s): product - film \& paper

Product: Eastman N.C. Film

Color or Black and White:

Binder: 1909-1913 Ad Books 3 of 6

Year: 1909

Slogan: The Dependability of Eastman NC Film

Subject(s): product - film \& paper

Product: Eastman N.C. Film

Color or Black and White:

Binder: 1909-1913 Ad Books 3 of 6

Year: 1909

Slogan: Experts use the Eastman Plate Tank

Subject(s): product - darkroom

Product: Eastman Plate Tank

\section{Color or Black and White:}

Binder: $1909-1913$ Ad Books 3 of 6

Year: 1909

Slogan: The Eastman Studio Register System

Subject(s): product - misc.

Product: Eastman Studio Register System

Color or Black and White:

Binder: 1909-1913 Ad Books 3 of 6

Year: 1909

Slogan: Enlargements are profitable Eastman's Permanent

Bromide

Subject(s): product - film \& paper

Product: Eastman's Permanent Bromide

Color or Black and White:

Binder: 1909-1913 Ad Books 3 of 6

Year: 1909

Slogan: Make your better grade enlargements on Eastman's Royal Bromide

Subject(s): product - film \& paper

Product: Eastman's Royal Bromide

Color or Black and White

Binder: 1909-1913 Ad Books 3 of 6

Year: 1909

Slogan: Eastman's Sepia Paper

Subject(s): product - film \& paper

Product: Eastman's Sepia Paper

Color or Black and White:

Binder: 1909-1913 Ad Books 3 of 6

Year: 1909

Slogan: Kodak Film Tank "The Daylight Dark-Room"

Subject(s): product - darkroom

Product: Kodak film tank

Color or Black and White: 
Binder: 1909-1913 Ad Books 3 of 6

Year: 1909

Slogan: Protect the Kodak quality of your Kodak negatives by developing them in the Kodak Film Tank

Subject(s): product - darkroom

Product: Kodak film tank

Color or Black and White:

Binder: 1909-1913 Ad Books 3 of 6

Year: 1909

Slogan: Greatest convenience; best results in the use of The

Kodak film tank

Subject(s): product - darkroom

Product: Kodak film tank

Color or Black and White:

Binder: 1909-1913 Ad Books 3 of 6

Year: 1909

Slogan: It's pleasure all the way when you develop with the Kodak Film Tank

Subject(s): product - darkroom

Product: Kodak film tank

Color or Black and White:

Binder: 1909-1913 Ad Books 3 of 6

Year: 1909

Slogan: Two names that you have confidence in-- Kodak and Zeiss

Subject(s): product - camera

Product: Kodak lenses

Color or Black and White:

Binder: 1909-1913 Ad Books 3 of 6

Year: 1909

Slogan: The R.O.C. Post Card Printer

Subject(s): product - film \& paper

Product: R.O.C. Post Card Printer

Description / Notes: Price: $\$ 7.50$
Binder: 1909-1913 Ad Books 3 of 6

Year: 1909

Slogan: The Rounds Print Washer

Subject(s): product - darkroom

Product: Rounds Print Washer

Description / Notes: Price: $\$ 10.00$

Binder: 1909-1913 Ad Books 3 of 6

Year: 1909

Slogan: Seed Plates

Subject(s): product - film \& paper

Product: Seed Dry Plates

Color or Black and White

Binder: 1909-1913 Ad Books 3 of 6

Year: 1909

Slogan: Even in the hottest weather Standard Thermic Plates

Subject(s): product - film \& paper

Product: Standard Dry Plate

Color or Black and White:

Binder: 1909-1913 Ad Books 3 of 6

Year: 1909

Slogan: No blisters--no frills when you use Standard Thermic Plates

Subject(s): product - film \& paper

Product: Standard Dry Plate

Color or Black and White:

Binder: 1909-1913 Ad Books 3 of 6

Year: 1909

Slogan: Use Standard Thermic Plates

Subject(s): product - film \& paper

Product: Standard Dry Plate

Color or Black and White:

Binder: 1909-1913 Ad Books 3 of 6

Year: 1909

Slogan: For speed and a truthful rendering of color values select 
Standard Polychrome Plates

Subject(s): product - film \& paper

Product: Standard Dry Plate

Color or Black and White:

Binder: 1909-1913 Ad Books 3 of 6

Year: 1909

Slogan: The Eastman Timer

Subject(s): product - darkroom

Product: The Eastman Timer

Description / Notes: Price: $\$ 2.00$

Binder: 1909-1913 Ad Books 3 of 6

Year: 1909

Slogan: The Eastman Trimming Board

Subject(s): product - misc.

Product: The Eastman Trimming Board

Color or Black and White:

Binder: 1909-1913 Ad Books 3 of 6

Year: 1909

Slogan: For correctly developed, clean negatives use The Kodak

Film Tank

Subject(s): product - darkroom

Product: The Kodak Film Tank

Color or Black and White:

Binder: 1909-1913 Ad Books 3 of 6

Year: 1909

Slogan: Velox

Subject(s): product - film \& paper

Product: Velox paper

Color or Black and White:

Binder: 1909-1913 Ad Books 3 of 6

Year: 1909

Slogan: For good prints from all negatives use Velox

Subject(s): product - film \& paper

Product: Velox paper

\section{Color or Black and White}

Binder: 1909-1913 Ad Books 3 of 6

Year: 1909

Slogan: Velox Post Cards

Subject(s): product - film \& paper

Product: Velox Post Cards

Color or Black and White

Binder: 1909-1913 Ad Books 4 of 6

Year: 1911

Slogan: Use Angelo and raise your standard

Subject(s): product - film \& paper

Product: Angelo paper

Color or Black and White:

Binder: $1909-1913$ Ad Books 4 of 6

Year: 1911

Slogan: Quality, Certainty of Results, Ease of Manipulation

Subject(s): product - film \& paper

Product: Aristo Gold Post Cards

Color or Black and White:

Binder: 1909-1913 Ad Books 4 of 6

Year: Oct-11

Slogan: Extreme Simplicity Carbon Quality Aristo Gold Paper Post

Cards

Subject(s): product - film \& paper

Product: Aristo Gold Post Cards

Color or Black and White:

Binder: 1909-1913 Ad Books 4 of 6

Year: 1911

Slogan: Quality--tone--beautiful surface and finish are yours when you use Aristo

Subject(s): product - film \& paper

Product: Aristo paper

Color or Black and White: 
Binder: 1909-1913 Ad Books 4 of 6

Year: 1911

Slogan: For Excellence of tone--for delicacy of texture--for general satisfaction Aristo

Subject(s): product - film \& paper

Product: Aristo paper

Color or Black and White:

Binder: $1909-1913$ Ad Books 4 of 6

Year: 1911

Slogan: For Permanent Prints--certain results and pleased

customers--use Aristo

Subject(s): product - film \& paper

Product: Aristo paper

Color or Black and White:

Binder: $1909-1913$ Ad Books 4 of 6

Year: 1911

Slogan: If your prints look almost as good as Aristo, use Aristo

Subject(s): product - film \& paper

Product: Aristo paper

Color or Black and White:

Binder: 1909-1913 Ad Books 4 of 6

Year: 1911

Slogan: A Permanent Success--Aristo

Subject(s): product - film \& paper

Product: Aristo paper

Color or Black and White:

Binder: 1909-1913 Ad Books 4 of 6

Year: 1911

Slogan: Aristo continues to make permanent customers.

Subject(s): product - film \& paper

Product: Aristo paper

Color or Black and White:

Binder: 1909-1913 Ad Books 4 of 6

Year: 1911
Slogan: Artura Carbon Black

Subject(s): product - film \& paper

Product: Artura Carbon Black

Color or Black and White:

Binder: 1909-1913 Ad Books 4 of 6

Year: 1911

Slogan: Make Sure; use the genuinely good: Artura Iris

Subject(s): product - film \& paper

Product: Artura Iris Paper

Color or Black and White:

Binder: 1909-1913 Ad Books 4 of 6

Year: 1911

Slogan: Use the paper of known excellence: Artura Iris

Subject(s): product - film \& paper

Product: Artura Iris Paper

Color or Black and White:

Binder: 1909-1913 Ad Books 4 of 6

Year: 1911

Slogan: Artura

Subject(s): product - film \& paper

Product: Artura Paper

Color or Black and White:

Binder: 1909-1913 Ad Books 4 of 6

Year: 1911

Slogan: A success built on Quality Atrura

Subject(s): product - film \& paper

Product: Artura Paper

Color or Black and White:

Binder: 1909-1913 Ad Books 4 of 6

Year: 1911

Slogan: "Just as good as Artura"

Subject(s): product - film \& paper

Product: Artura Paper

Color or Black and White 
Binder: 1909-1913 Ad Books 4 of 6

Year: 1911

Slogan: Artura Success

Subject(s): product - film \& paper

Product: Artura Paper

Color or Black and White:

Binder: 1909-1913 Ad Books 4 of 6

Year: 1911

Slogan: Superior Sepias on an inexpensive paper: Azo

Subject(s): product - film \& paper

Product: Azo paper

Color or Black and White:

Binder: 1909-1913 Ad Books 4 of 6

Year: 1911

Slogan: Azo Sepias

Subject(s): product - film \& paper

Product: Azo paper

Color or Black and White:

Binder: 1909-1913 Ad Books 4 of 6

Year: 1911

Slogan: Enlarge your business by making Carbon Black Artura

Subject(s): product - film \& paper

Product: Carbon Black Artura

Color or Black and White:

Binder: 1909-1913 Ad Books 4 of 6

Year: 1911

Slogan: Eastman EB and ES Platinum

Subject(s): product - film \& paper

Product: Eastman EB and ES Platinum

Color or Black and White:

Binder: 1909-1913 Ad Books 4 of 6

Year: 1911

Slogan: Velvety surface--correct sepia tone and perfect printing quality are factors in the growing success of Eastman ES Platinum

Subject(s): product - film \& paper

Product: Eastman ES Platinum

Color or Black and White:

Binder: 1909-1913 Ad Books 4 of 6

Year: 1911

Slogan: The importance of using pure and active chemicals cannot

be overestimated. The dependable kind--tested and analyzed carry

this mark of distinction on the label

Subject(s): product - Chemicals

Product: Eastman Kodak Company Tested Chemicals

Color or Black and White:

Binder: $1909-1913$ Ad Books 4 of 6

Year: 1911

Slogan: New Name Same Goods

Subject(s): product - Chemicals

Product: Eastman Kodak Company Tested Chemicals

Description / Notes: Switching the chemical Enol to the name Elon

Binder: 1909-1913 Ad Books 4 of 6

Year: 1911

Slogan: Elon

Subject(s): product - Chemicals

Product: Eastman Kodak Company Tested Chemicals

Color or Black and White:

Binder: 1909-1913 Ad Books 4 of 6

Year: 1911

Slogan: It stays where you put it. Eastman Permanent Crystal

Pyro

Subject(s): product - Chemicals

Product: Eastman Kodak Company Tested Chemicals

Color or Black and White:

Binder: 1909-1913 Ad Books 4 of 6

Year: 1911 
Slogan: Fill your shelves with The Best--

Subject(s): product - Chemicals

Product: Eastman Kodak Company Tested Chemicals

Color or Black and White:

Binder: 1909-1913 Ad Books 4 of 6

Year: 1911

Slogan: The Proof of Quality continued use of Elon

Subject(s): product - Chemicals

Product: Eastman Kodak Company Tested Chemicals

Color or Black and White:

Binder: 1909-1913 Ad Books 4 of 6

Year: 1911

Slogan: Insure your results

Subject(s): product - Chemicals

Product: Eastman Kodak Company Tested Chemicals

Color or Black and White:

Binder: 1909-1913 Ad Books 4 of 6

Year: 1911

Slogan: This seal is the stamp of approval of our chemical testing

department; It means, tested chemically--tested photographically-

-right for our use--right for your use

Subject(s): product - Chemicals

Product: Eastman Kodak Company Tested Chemicals

Color or Black and White:

Binder: $1909-1913$ Ad Books 4 of 6

Year: 1911

Slogan: We must be certain of our chemicals--therefore we test

them. You can also be certain of your chemicals--because we test them. This seal tells.

Subject(s): product - Chemicals

Product: Eastman Kodak Company Tested Chemicals

Color or Black and White:

Binder: 1909-1913 Ad Books 4 of 6

Year: Oct-11
Slogan: Economical Clean and Convenient

Subject(s): product - Chemicals

Product: Eastman Kodak Company Tested Chemicals

Color or Black and White:

Binder: 1909-1913 Ad Books 4 of 6

Year: 1911

Slogan: Full Kodak efficiency is realized only in the use of

Eastman NC Film

Subject(s): product - film \& paper

Product: Eastman N.C. Film

Color or Black and White:

Binder: 1909-1913 Ad Books 4 of 6

Year: Oct-11

Slogan: Order Eastman Tested Chemicals and be certain

Subject(s): product - Chemicals

Product: Eastman Permanent Crystal Pyro

Color or Black and White:

Binder: 1909-1913 Ad Books 4 of 6

Year: 1911

Slogan: The Eastman Plate Tank

Subject(s): product - darkroom

Product: Eastman Plate Tank

Color or Black and White:

Binder: 1909-1913 Ad Books 4 of 6

Year: 1911

Slogan: Twelve Perfectly Developed Negatives Every Time

Subject(s): product - darkroom

Product: Eastman Plate Tank

Color or Black and White:

Binder: 1909-1913 Ad Books 4 of 6

Year: 1911

Slogan: The proof of the tank is in the quality of the negative. The

Eastman Plate Tank

Subject(s): product - darkroom 
Product: Eastman Plate Tank

Color or Black and White:

Binder: 1909-1913 Ad Books 4 of 6 Year: 1911

Slogan: Use the Kodak Film Tank

Subject(s): product - darkroom

Product: Kodak film tank

Color or Black and White:

Binder: 1909-1913 Ad Books 4 of 6

Year: 1911

Slogan: Take a Kodak film tank

Subject(s): product - darkroom

Product: Kodak film tank

Color or Black and White:

Binder: 1909-1913 Ad Books 4 of 6

Year: 1911

Slogan: Properly Orthochromatic Kodak NC Film

Subject(s): product - film \& paper

Product: Kodak N.C. Film

Color or Black and White:

Binder: 1909-1913 Ad Books 4 of 6

Year: 1911

Slogan: Kodak Velvet Green Paper

Subject(s): product - film \& paper

Product: Kodak Velvet Green Paper

Color or Black and White:

Binder: 1909-1913 Ad Books 4 of 6 Year: 1911

Slogan: In Just Three Minutes

Subject(s): product - film \& paper

Product: Kodak Velvet Green Paper

Color or Black and White:

Binder: 1909-1913 Ad Books 4 of 6
Year: 1911

Slogan: Your Vacation Postals will be more pleasing on Kodak

Velvet Green Paper

Subject(s): product - film \& paper

Product: Kodak Velvet Green Paper

Color or Black and White:

Binder: 1909-1913 Ad Books 4 of 6

Year: 1911

Slogan: Paragon Border Negatives

Subject(s): product - film \& paper

Product: Paragon Border Negatives

Color or Black and White:

Binder: 1909-1913 Ad Books 4 of 6

Year: 1911

Slogan: Seed CV Plates

Subject(s): product - film \& paper

Product: Seed Dry Plates

Color or Black and White:

Binder: 1909-1913 Ad Books 4 of 6

Year: 1911

Slogan: Double the Speed of the Seed 27

Subject(s): product - film \& paper

Product: Seed Dry Plates

Color or Black and White:

Binder: 1909-1913 Ad Books 4 of 6

Year: 1911

Slogan: Does this interest you?

Subject(s): product - film \& paper

Product: Seed Dry Plates

Color or Black and White:

Binder: 1909-1913 Ad Books 4 of 6

Year: 1911

Slogan: Seed Gilt Edge Plates

Subject(s): product - film \& paper 
Product: Seed Dry Plates

Color or Black and White:

Binder: 1909-1913 Ad Books 4 of 6

Year: 1911

Slogan: --

Subject(s): product - film \& paper

Product: Seed Dry Plates

Description / Notes: Letter from Rudolf Diihrtoop, Berlin. To a Mr. Fell at Kodak. Explaining that he has used Seed plates since 1904 and is "perfectly satisfied with the qualities"

Binder: 1909-1913 Ad Books 4 of 6

Year: Oct-11

Slogan: Seed The Standard of Excellence

Subject(s): product - film \& paper

Product: Seed Dry Plates

Color or Black and White:

Binder: 1909-1913 Ad Books 4 of 6

Year: 1911

Slogan: If it's in the negative, you get it in the Velox print.

Subject(s): product - film \& paper

Product: Velox paper

Color or Black and White:

Binder: 1909-1913 Ad Books 4 of 6

Year: 1911

Slogan: Make your album of Vacation pictures an album of Velox pictures

Subject(s): product - film \& paper

Product: Velox paper

Color or Black and White:

Binder: 1909-1913 Ad Books 4 of 6

Year: Oct-11

Slogan: Select Velox

Subject(s): product - film \& paper

Product: Velox paper

\section{Color or Black and White}

Binder: 1909-1913 Ad Books 5 of 6

Year: Oct-12

Slogan: A photographic Education

Subject(s): product - misc.

Product: "How to Make Good Pictures" booklet

Description / Notes: Price: $\$ 0.25$

Binder: 1909-1913 Ad Books 5 of 6

Year: 1912

Slogan: An Enviable Reputation. The Permanency of Aristo

Subject(s): product - film \& paper

Product: Aristo paper

Color or Black and White:

Binder: 1909-1913 Ad Books 5 of 6

Year: Nov-11

Slogan: Twenty-two years of pleased customers is the record of Aristo

Subject(s): product - film \& paper

Product: Aristo paper

Color or Black and White:

Binder: 1909-1913 Ad Books 5 of 6

Year: Jan-12

Slogan: Aristo Facts

Subject(s): product - film \& paper

Product: Aristo paper

Color or Black and White

Binder: 1909-1913 Ad Books 5 of 6

Year: Jun-12

Slogan: Pin your faith to the permanency, quality and reliability of Aristo

Subject(s): product - film \& paper

Product: Aristo paper

Color or Black and White: 
Binder: 1909-1913 Ad Books 5 of 6

Year: Jan-12

Slogan: The Reasons for making enlargements are many, the good papers, few. Artura Carbon Black

Subject(s): product - film \& paper

Product: Artura Carbon Black

Color or Black and White:

Binder: 1909-1913 Ad Books 5 of 6

Year: 1912

Slogan: The Proof of quality is in the using: Artura

Subject(s): product - film \& paper

Product: Artura Paper

Color or Black and White:

Binder: 1909-1913 Ad Books 5 of 6

Year: 1912

Slogan: Artura prints are the best advertisements for you.

Subject(s): product - film \& paper

Product: Artura Paper

Color or Black and White:

Binder: 1909-1913 Ad Books 5 of 6

Year: 1912

Slogan: The Holiday Rush loses it's terrors when you use Artura

Subject(s): product - film \& paper

Product: Artura paper

Color or Black and White:

Binder: 1909-1913 Ad Books 5 of 6

Year: Jun-12

Slogan: Watch the work of the man who uses Artura

Subject(s): product - film \& paper

Product: Artura Paper

Color or Black and White:

Binder: 1909-1913 Ad Books 5 of 6

Year: Aug-12

Slogan: Enlargements on Artura Carbon Black
Subject(s): product - film \& paper

Product: Artura Paper

Color or Black and White:

Binder: 1909-1913 Ad Books 5 of 6

Year: Sep-12

Slogan: In Quality Artura Knows no Equal

Subject(s): product - film \& paper

Product: Artura Paper

Color or Black and White:

Binder: $1909-1913$ Ad Books 5 of 6

Year: Dec-12

Slogan: Artura Carbon Black Buff Stock

Subject(s): product - film \& paper

Product: Artura paper

Color or Black and White:

Binder: 1909-1913 Ad Books 5 of 6

Year: 1912

Slogan: The New Artura Printer

Subject(s): product - darkroom

Product: Artura Printer

Description / Notes: Complete with special arc lamp $11 \times 14$ printer price: $\$ 75.00$. Without lamp: $\$ 55.00 .20 \times 24$ with special arc lamp: $\$ 100.00$. Without lamp: $\$ 80.00$

Binder: 1909-1913 Ad Books 5 of 6

Year: Jun-12

Slogan: Where the one essential is Quality the one paper is Artura

Subject(s): product - film \& paper

Product: Artura Printer

Color or Black and White:

Binder: 1909-1913 Ad Books 5 of 6

Year: Jan-12

Slogan: Azo for the Amateur finisher

Subject(s): product - film \& paper

Product: Azo paper 


\section{Color or Black and White:}

Binder: 1909-1913 Ad Books 5 of 6 Year: Nov-11

Slogan: Eastman EB or ES Platinum

Subject(s): product - film \& paper

Product: Eastman EB and ES Platinum

Color or Black and White:

Binder: 1909-1913 Ad Books 5 of 6

Year: Dec-12

Slogan: Effective Quality

Subject(s): product - film \& paper

Product: Eastman EB and ES Platinum

Color or Black and White:

Binder: 1909-1913 Ad Books 5 of 6

Year: 1912

Slogan: Their use is your protection

Subject(s): product - Chemicals

Product: Eastman Kodak Company Tested Chemicals

Color or Black and White:

Binder: 1909-1913 Ad Books 5 of 6

Year: 1912

Slogan: Chemical Facts.

Subject(s): product - Chemicals

Product: Eastman Kodak Company Tested Chemicals

Color or Black and White:

Binder: 1909-1913 Ad Books 5 of 6

Year: 1912

Slogan: Chemical Logic

Subject(s): product - Chemicals

Product: Eastman Kodak Company Tested Chemicals

Color or Black and White:

Binder: 1909-1913 Ad Books 5 of 6

Year: 1912
Slogan: E.K. Tested Chemicals cost you no more.

Subject(s): product - Chemicals

Product: Eastman Kodak Company Tested Chemicals

Color or Black and White:

Binder: 1909-1913 Ad Books 5 of 6

Year: 1912

Slogan: Proof of the Pudding

Subject(s): product - Chemicals

Product: Eastman Kodak Company Tested Chemicals

Color or Black and White:

Binder: 1909-1913 Ad Books 5 of 6

Year: 1912

Slogan: Eastman Tested Acetic Acid

Subject(s): product - Chemicals

Product: Eastman Kodak Company Tested Chemicals

Color or Black and White:

Binder: 1909-1913 Ad Books 5 of 6

Year: Nov-11

Slogan: $98.2 \%$ Pure

Subject(s): product - Chemicals

Product: Eastman Kodak Company Tested Chemicals

Color or Black and White:

Binder: 1909-1913 Ad Books 5 of 6

Year: Nov-11

Slogan: Your Assurance of uniform quality

Subject(s): product - Chemicals

Product: Eastman Kodak Company Tested Chemicals

Color or Black and White:

Binder: 1909-1913 Ad Books 5 of 6

Year: Dec-11

Slogan: The Mark of Distinction

Subject(s): product - Chemicals

Product: Eastman Kodak Company Tested Chemicals

Color or Black and White: 
Binder: 1909-1913 Ad Books 5 of 6

Year: Jan-12

Slogan: Roylon A vigorous developing agent for use in connection with Hydrochinon

Subject(s): product - Chemicals

Product: Eastman Kodak Company Tested Chemicals Description / Notes: Price 1 ounce bottle: $\$ 0.60 .1 / 4$ pound:

$\$ 2.25 .1 / 2$ pound: $\$ 4.25 .1$ pound: $\$ 8.00$

Binder: 1909-1913 Ad Books 5 of 6

Year: Jun-12

Slogan: A World of Importance to Photographers "Tested"

Subject(s): product - Chemicals

Product: Eastman Kodak Company Tested Chemicals

Color or Black and White:

Binder: 1909-1913 Ad Books 5 of 6

Year: Aug-12

Slogan: When you say chemicals, say also the kind.

Subject(s): product - Chemicals

Product: Eastman Kodak Company Tested Chemicals

Color or Black and White:

Binder: 1909-1913 Ad Books 5 of 6

Year: Sep-12

Slogan: You Save 20\%

Subject(s): product - Chemicals

Product: Eastman Kodak Company Tested Chemicals

Color or Black and White:

Binder: 1909-1913 Ad Books 5 of 6

Year: Dec-12

Slogan: Get Acquainted with the mark of "Chemical Certainty."

Subject(s): product - Chemicals

Product: Eastman Kodak Company Tested Chemicals

Color or Black and White:

Binder: 1909-1913 Ad Books 5 of 6
Year: 1912

Slogan: To get the best possible print--get acquainted with Velox

Subject(s): product - film \& paper

Product: Eastman Kodak Company Tested Chemicals

Color or Black and White

Binder: 1909-1913 Ad Books 5 of 6

Year: Dec-11

Slogan: Use the Eastman Plate Tank

Subject(s): product - darkroom

Product: Eastman Plate Tank

Color or Black and White:

Binder: 1909-1913 Ad Books 5 of 6

Year: Jun-12

Slogan: Less work at the retouching desk when you use The Eastman Plate Tank

Subject(s): product - darkroom

Product: Eastman Plate Tank

Color or Black and White:

Binder: 1909-1913 Ad Books 5 of 6

Year: Aug-12

Slogan: In hot weather there's no dread of developing--no fear of the result when the Eastman Plate Tank does the work.

Subject(s): product - darkroom

Product: Eastman Plate Tank

Color or Black

Binder: 1909-1913 Ad Books 5 of 6

Year: 1912

Slogan: Overcome hot weather troubles with The Eastman Plate Tank

Subject(s): product - film \& paper

Product: Eastman Plate Tank

Color or Black and White:

Binder: 1909-1913 Ad Books 5 of 6

Year: Nov-11 
Slogan: Eastman Studio Reflector

Subject(s): product - misc.

Product: Eastman Studio Reflector

Description / Notes: Price: $\$ 12.00$

Binder: 1909-1913 Ad Books 5 of 6

Year: 1912

Slogan: Systematize your business with an Eastman Studio

Register System

Subject(s): product - misc.

Product: Eastman Studio Register System

Description / Notes: Two cases with cards price: $\$ 5.00$

Binder: 1909-1913 Ad Books 5 of 6

Year: 1912

Slogan: Good Times At Home with A Kodak

Subject(s): product - camera

Product: Kodak cameras, portrait attachment

Description / Notes: article.

Binder: 1909-1913 Ad Books 5 of 6

Year: Dec-11

Slogan: Be Sure your film is Kodak Film

Subject(s): product - film \& paper

Product: Kodak film

Color or Black and White:

Binder: 1909-1913 Ad Books 5 of 6

Year: 1912

Slogan: The Kodak Film Tank way is the simplest way--the best way

Subject(s): product - darkroom

Text: Tank development is a scientific fact past argument.

Examine a film developed in the Kodak Film Tank. The developer,

so balanced as to be slow in it's action, searches out all the exposed silver and produces an image of fine grain with ful delicacy and detail. There is no fog. Then there is the convenience of being able to develop anywhere without a dark-room.

Implied Audience: women / genera
Product: Kodak film tank

Photo or Illustration Based: photo

Color or Black and White: bw

Description / Notes: Photo of a woman in an apron holding a roll of film -- same image as before, different text.

Binder: 1909-1913 Ad Books 5 of 6

Year: 1912

Slogan: Don't Wait Until You Get Home The Kodak Film Tank

Subject(s): product - darkroom

Product: Kodak film tank

Color or Black and White:

Binder: 1909-1913 Ad Books 5 of 6

Year: 1912

Slogan: Add Zest to picture making at home

Subject(s): product - darkroom

Product: Kodak Film Tank

Color or Black and White:

Binder: 1909-1913 Ad Books 5 of 6

Year: Dec-11

Slogan: Developing is a pleasure with the Kodak Film Tank

Subject(s): product - darkroom

Product: Kodak film tank

Color or Black and White:

Binder: 1909-1913 Ad Books 5 of 6

Year: Oct-12

Slogan: Double your good times

Subject(s): product - darkroom

Product: Kodak film tank

Color or Black and White:

Binder: 1909-1913 Ad Books 5 of 6

Year: Dec-12

Slogan: Doubly worth while

Subject(s): product - darkroom

Product: Kodak film tank 


\section{Color or Black and White:}

Binder: 1909-1913 Ad Books 5 of 6

Year: Dec-12

Slogan: All Good Negatives

Subject(s): product - darkroom

Product: Kodak film tank

Color or Black and White:

Binder: 1909-1913 Ad Books 5 of 6

Year: 1912

Slogan: The Kodak Film Tank way is the simplest way--the best way

Subject(s): Two boys

Text: Added to the pleasure of doing ones own developing is the satisfaction of doing it better when the Kodak Film Tank is used.

The dark-room with its many causes for faulty manipulation is

eliminated. One may develop anywhere where a Kodak Film Tank.

The Experience is in the Tank

Implied Audience: parents/children

Product: Kodak film tank

Photo or Illustration Based: photo

Color or Black and White: bw

Description / Notes: Photo of two boys, at a window holding up film. Tank is on the table.

Binder: 1909-1913 Ad Books 5 of 6

Year: 1912

Slogan: The Kodak Film Tank Way

Subject(s): woman

Text: The Pleasure is all yours when you develop the Kodak Film

Tank Way. And there's the satisfaction of doing the work better-securing clean negatives free from fog, scratches and finger

marks. You can develop anywhere with a Kodak Film Tank. The experience is in the tank.

Implied Audience: women / general

Product: Kodak film tank

Photo or Illustration Based: photo

Color or Black and White: bw
Description / Notes: Photo of a woman in a sailor dress sitting at a table, developing in the tank.

Binder: 1909-1913 Ad Books 5 of 6

Year: Jan-12

Slogan: The Kodak Film Tank way is the simplest way--the best

way

Subject(s): woman

Text: Negatives developed in the Kodak Film Tank are clean, are free from scratches and finger marks; they have more brilliancy, gradation, and detail, because free from fog. Then there is the convenience of being able to develop anywhere without a dark-

room

Implied Audience: women / general

Product: Kodak film tank

Photo or Illustration Based: photo

Color or Black and White: bw

Description / Notes: Photo of a woman in an apron holding up a roll of film. Tank is on the table.

Binder: 1909-1913 Ad Books 5 of 6

Year: Jun-12

Slogan: She has Confidence in the Kodak Film Tank Way

Subject(s): woman

Text: It's a pleasure for there's no dark-room, no stained fingers, no muss, and best of all, no uncertainty. Film Tank results are always better, for the negatives are clean, crisp and free from finger marks and fog. They make better prints too. Take a Kodak Film Tank on that vacation trip. The experience is in the tank.

Implied Audience: women / general

Product: Kodak film tank

Photo or Illustration Based: photo

Color or Black and White: bw

Description / Notes: Photo of a woman in a sailor dress sitting at a table, developing in the tank. Same as above, but with different text.

Binder: 1909-1913 Ad Books 5 of 6

Year: 1912 
Slogan: The Quality of Carbon The Simplicity of Velox

Subject(s): product - film \& paper

Product: Kodak Velvet Green Paper

Color or Black and White:

Binder: 1909-1913 Ad Books 5 of 6

Year: 1912

Slogan: For the Occasional Print that Lends Variety Kodak Velvet

Green Paper

Subject(s): product - film \& paper

Product: Kodak Velvet Green Paper

Color or Black and White:

Binder: 1909-1913 Ad Books 5 of 6

Year: Aug-12

Slogan: You get nearer to the truth in Nature when the print is made on Kodak Velvet Green Paper

Subject(s): product - film \& paper

Product: Kodak Velvet Green Paper

Color or Black and White:

Binder: 1909-1913 Ad Books 5 of 6

Year: Oct-12

Slogan: "It's more real--more full of life"

Subject(s): product - film \& paper

Product: Kodak Velvet Green Paper

Color or Black and White:

Binder: 1909-1913 Ad Books 5 of 6

Year: Dec-12

Slogan: Prints out of the Ordinary--by ordinary methods

Subject(s): product - film \& paper

Product: Kodak Velvet Green Paper

Color or Black and White:

Binder: 1909-1913 Ad Books 5 of 6

Year: 1912

Slogan: Roylon A New Developing Agent

Subject(s): product - Chemicals
Product: Roylon Developing Agent

Description / Notes: Price 1 ounce bottle: $\$ 0.60 .1 / 4$ pound:

$\$ 2.25 .1 / 2$ pound: $\$ 4.25 .1$ pound: $\$ 8.00$

Binder: 1909-1913 Ad Books 5 of 6

Year: 1912

Slogan: The Advantages of Roylon

Subject(s): product - darkroom

Product: Roylon Developing Agent

Color or Black and White:

Binder: 1909-1913 Ad Books 5 of 6

Year: 1912

Slogan: Use Seed Plates

Subject(s): product - film \& paper

Product: Seed Dry Plates

Color or Black and White:

Binder: 1909-1913 Ad Books 5 of 6

Year: 1912

Slogan: A Seed Plate for Every Purpose

Subject(s): product - film \& paper

Product: Seed Dry Plates

Color or Black and White:

Binder: 1909-1913 Ad Books 5 of 6

Year: 1912

Slogan: If you would get action

Subject(s): product - film \& paper

Product: Seed Dry Plates

Color or Black and White:

Binder: 1909-1913 Ad Books 5 of 6

Year: Dec-11

Slogan: Seed Plates For Pictorial Work

Subject(s): product - film \& paper

Product: Seed Dry Plates

Color or Black and White: 
Binder: 1909-1913 Ad Books 5 of 6

Year: Jan-12

Slogan: Seed Gilt Edge Plates For Speed Work

Subject(s): product - film \& paper

Product: Seed Dry Plates

Color or Black and White:

Binder: 1909-1913 Ad Books 5 of 6

Year: Aug-12

Slogan: Seed characteristics are Quality characteristics

Subject(s): product - film \& paper

Product: Seed Dry Plates

Color or Black and White:

Binder: 1909-1913 Ad Books 5 of 6

Year: Oct-12

Slogan: Seed Quality is Printing Quality

Subject(s): product - film \& paper

Product: Seed Dry Plates

Color or Black and White:

Binder: 1909-1913 Ad Books 5 of 6

Year: Dec-12

Slogan: Speed Plus Quality

Subject(s): product - film \& paper

Product: Seed Dry Plates

Color or Black and White:

Binder: 1909-1913 Ad Books 5 of 6

Year: Dec-12

Slogan: Winter Sports

Subject(s): product - film \& paper

Product: Seed Dry Plates

Color or Black and White:

Binder: 1909-1913 Ad Books 5 of 6

Year: Jan-12

Slogan: Tozol

Subject(s): product - darkroom
Product: Tozol Developing Agent

Description / Notes: Price 1 ounce bottle: $\$ 0.20 .1 / 4$ pound:

$\$ 0.70 .1 / 2$ pound: $\$ 1.30 .1$ pound: $\$ 2.50$

Binder: 1909-1913 Ad Books 5 of 6

Year: Aug-12

Slogan: Tozol Simplifies the Process by Making it Shorter

Subject(s): product - darkroom

Product: Tozol Developing Agent

Color or Black and White:

Binder: $1909-1913$ Ad Books 5 of 6

Year: 1912

Slogan: Tozol The simple developing agent for developing papers

Subject(s): product - film \& paper

Product: Tozol Developing Agent

Color or Black and White:

Binder: 1909-1913 Ad Books 5 of 6

Year: Sep-12

Slogan: The Best Amateurs use Velox

Subject(s): product - film \& paper

Product: Velox

Color or Black and White:

Binder: 1909-1913 Ad Books 5 of 6

Year: Dec-12

Slogan: Resolve to use Velox. Your negatives are worth it.

Subject(s): product - film \& paper

Product: Velox

Color or Black and White:

Binder: 1909-1913 Ad Books 5 of 6

Year: 1912

Slogan: After the vacation there's Velox

Subject(s): product - film \& paper

Product: Velox paper

Color or Black and White: 
Binder: 1909-1913 Ad Books 5 of 6

Year: Dec-11

Slogan: Prints by Gaslight

Subject(s): product - film \& paper

Product: Velox paper

Color or Black and White:

Binder: 1909-1913 Ad Books 5 of 6

Year: Jan-12

Slogan: Prints by Gaslight Velox

Subject(s): product - film \& paper

Product: Velox paper

Color or Black and White:

Binder: 1909-1913 Ad Books 5 of 6

Year: Dec-12

Slogan: The result you work for is the print.

Subject(s): product - film \& paper

Product: Velox paper

Color or Black and White:

Binder: 1909-1913 Ad Books 5 of 6

Year: Oct-12

Slogan: Zelta Matte-Surface, Ready Sensitized, Four Grades.

Subject(s): product - film \& paper

Product: Zelta Paper

Color or Black and White:

Binder: 1909-1913 Ad Books 6 of 6

Year: Jul-13

Slogan: A Book of practice, not theory. 160 pages of "What to do and why."

Subject(s): product - misc.

Product: "How to Make Good Pictures" booklet

Description / Notes: Booklet price: $\$ 0.25$. Library edition, cloth covers: $\$ 1.00$

Binder: 1909-1913 Ad Books 6 of 6

Year: 1913
Slogan: A Suggestion to the user of Angelo Sepia

Subject(s): product - film \& paper

Product: Angelo paper

Color or Black and White:

Binder: 1909-1913 Ad Books 6 of 6

Year: Oct-13

Slogan: Make contact quality enlargements on Artura Carbon

Black

Subject(s): product - film \& paper

Product: Artura Carbon Black

Color or Black and White:

Binder: 1909-1913 Ad Books 6 of 6

Year: Feb-13

Slogan: Artura Knows no Equal

Subject(s): product - film \& paper

Product: Artura Paper

Color or Black and White:

Binder: 1909-1913 Ad Books 6 of 6

Year: Apr-13

Slogan: Use the paper that has no equal

Subject(s): product - film \& paper

Product: Artura paper

Color or Black and White:

Binder: 1909-1913 Ad Books 6 of 6

Year: May-13

Slogan: Make your enlargements on the New Artura Carbon Black Buff Stock

Subject(s): product - film \& paper

Product: Artura Paper

Color or Black and White:

Binder: 1909-1913 Ad Books 6 of 6

Year: Jun-13

Slogan: The proof of superiority--the number of successful photographers who use Artura 
Subject(s): product - film \& paper

Product: Artura Paper

Color or Black and White:

Binder: 1909-1913 Ad Books 6 of 6

Year: Aug-13

Slogan: Reproduce the quality of the negative in the print

Subject(s): product - film \& paper

Product: Artura Paper

Color or Black and White:

Binder: 1909-1913 Ad Books 6 of 6

Year: Sep-13

Slogan: Back up your skill in lighting and posing with Artura

Subject(s): product - film \& paper

Product: Artura Paper

Color or Black and White:

Binder: 1909-1913 Ad Books 6 of 6

Year: Sep-13

Slogan: Give your customer the best print you can make from

every negative

Subject(s): product - film \& paper

Product: Artura Paper

Color or Black and White:

Binder: 1909-1913 Ad Books 6 of 6

Year: 1913

Slogan: Enlargements by Daylight

Subject(s): product - camera

Product: Brownie Enlarging Camera

Description / Notes: No. 2 price: $\$ 2.00$. No. 3 price: $\$ 3.00$. No.

4 Price: $\$ 4.00$

Binder: 1909-1913 Ad Books 6 of 6

Year: Oct-13

Slogan: Pictures that tell their story in large type.

Subject(s): product - darkroom

Product: Brownie Enlarging Camera
Description / Notes: Article.

Binder: 1909-1913 Ad Books 6 of 6

Year: Oct-13

Slogan: Make the print on platinum that is different

Subject(s): product - film \& paper

Product: Eastman EB and ES Platinum

Color or Black and White:

Binder: 1909-1913 Ad Books 6 of 6

Year: Sep-13

Slogan: Flash Fun / The Charm of Color

Subject(s): product - misc.

Product: Eastman Flash Sheets / Velox Transparent Water color stamps

Description / Notes: Article.

Binder: 1909-1913 Ad Books 6 of 6

Year: Jun-13

Slogan: Eastman Home Portrait Reflector

Subject(s): product - misc.

Product: Eastman Home Portrait Reflector

Color or Black and White:

Binder: 1909-1913 Ad Books 6 of 6

Year: 1913

Slogan: The Element of Certainty

Subject(s): product - Chemicals

Product: Eastman Kodak Company Tested Chemicals

Color or Black and White:

Binder: 1909-1913 Ad Books 6 of 6

Year: Jan-13

Slogan: Our interest in your results

Subject(s): product - Chemicals

Product: Eastman Kodak Company Tested Chemicals

Color or Black and White:

Binder: 1909-1913 Ad Books 6 of 6 
Year: Feb-13

Slogan: It costs money to experiment--it costs less to be certain Subject(s): product - Chemicals

Product: Eastman Kodak Company Tested Chemicals

Color or Black and White:

Binder: 1909-1913 Ad Books 6 of 6

Year: Apr-13

Slogan: Successful photography necessitates the use of

Dependable Chemicals

Subject(s): product - Chemicals

Product: Eastman Kodak Company Tested Chemicals

Color or Black and White:

Binder: 1909-1913 Ad Books 6 of 6

Year: Jun-13

Slogan: The Condition of your chemicals in hot weather is of the greatest importance.

Subject(s): product - Chemicals

Product: Eastman Kodak Company Tested Chemicals

Color or Black and White:

Binder: 1909-1913 Ad Books 6 of 6

Year: Jul-13

Slogan: Specify "E.K. Tested."

Subject(s): product - Chemicals

Product: Eastman Kodak Company Tested Chemicals

Color or Black and White:

Binder: 1909-1913 Ad Books 6 of 6

Year: Jul-13

Slogan: Use Elon

Subject(s): product - Chemicals

Product: Eastman Kodak Company Tested Chemicals

Color or Black and White:

Binder: 1909-1913 Ad Books 6 of 6

Year: Aug-13

Slogan: It costs no more to be certain--specify "E.K. Tested"
Subject(s): product - Chemicals

Product: Eastman Kodak Company Tested Chemicals

Color or Black and White:

Binder: 1909-1913 Ad Books 6 of 6

Year: Oct-13

Slogan: Look for this seal on every package or bottle

Subject(s): product - Chemicals

Product: Eastman Kodak Company Tested Chemicals

Color or Black and White:

Binder: 1909-1913 Ad Books 6 of 6

Year: 1913

Slogan: Good Insurance

Subject(s): product - film \& paper

Product: Eastman Kodak Company Tested Chemicals

Color or Black and White:

Binder: 1909-1913 Ad Books 6 of 6

Year: Aug-13

Slogan: Systematize your business Eastman Loose Leaf Studio Register

Subject(s): product - misc

Product: Eastman Loose Leaf Studio Register

Description / Notes: Price: $\$ 5.00$

Binder: 1909-1913 Ad Books 6 of 6

Year: 1913

Slogan: More Than Convenience / "A Darkless Dark Room"

Subject(s): product - film \& paper

Product: Eastman N.C. Film / Kodak Tank Developer

Description / Notes: Article.

Binder: 1909-1913 Ad Books 6 of 6

Year: 1913

Slogan: Permanent

Subject(s): product - film \& paper

Product: Eastman Permanent Bromide

Description / Notes: A series of letters, 1894, 1902, 1906 from 
Geo. G. Rockwood talking about his endorsement of Eastman's Permanent Bromide

Binder: 1909-1913 Ad Books 6 of 6

Year: Jan-13

Slogan: The Eastman Plate Tank makes a shorter day's work a better day's work.

Subject(s): product - darkroom

Product: Eastman Plate Tank

Color or Black and White:

Binder: 1909-1913 Ad Books 6 of 6

Year: May-13

Slogan: It's printing quality that counts

Subject(s): product - film \& paper

Product: Eastman Plate Tank

Color or Black and White:

Binder: 1909-1913 Ad Books 6 of 6

Year: 1913

Slogan: Eastman Portrait Films

Subject(s): product - film \& paper

Product: Eastman Portrait Films

Description / Notes: Price: Same as Seed 30 plates.

Binder: 1909-1913 Ad Books 6 of 6

Year: Sep-13

Slogan: The Greatest advance since the advent of the dry plate--

Eastman Portrait Films

Subject(s): product - film \& paper

Product: Eastman Portrait Films

Color or Black and White:

Binder: 1909-1913 Ad Books 6 of 6

Year: Feb-13

Slogan: Eastman Portrait Lens

Subject(s): product - camera

Product: Eastman Portrait Lens

Description / Notes: Series A price: $\$ 60.00-\$ 120.00$ Series B
Price: $\$ 32.00-\$ 57.00$

Binder: 1909-1913 Ad Books 6 of 6

Year: Jul-13

Slogan: Kodaking from an Aeroplane. / Two Cameras in One

Subject(s): product - camera

Product: Kodak cameras

Description / Notes: Article. With photo by Jorge Allan.

Binder: 1909-1913 Ad Books 6 of 6

Year: 1913

Slogan: How a Kodak Coached a University Football Team /

Eastman Film Negative Album

Subject(s): product - camera

Product: Kodak cameras, Eastman Film Negative Album.

Description / Notes: Article.

Binder: 1909-1913 Ad Books 6 of 6

Year: 1913

Slogan: Monsen of the Desert

Subject(s): product - misc.

Product: Kodak film and papers.

Color or Black and White:

Binder: 1909-1913 Ad Books 6 of 6

Year: May-13

Slogan: Use a Kodak Film Tank

Subject(s): product - darkroom

Product: Kodak film tank

Color or Black and White:

Binder: 1909-1913 Ad Books 6 of 6

Year: Jun-13

Slogan: Take a Kodak Film Tank on the Vacation--bring the picture story home complete.

Subject(s): product - darkroom

Product: Kodak Film Tank

Color or Black and White: 
Binder: 1909-1913 Ad Books 6 of 6

Year: Feb-13

Slogan: Take a Look at a clean, snappy, tank-developed film. Subject(s): woman

Text: It's the satisfaction of work better done that adds so much to the pleasure of developing the Kodak Film Tank way. The

Experience is in the tank

Implied Audience: women / general

Product: Kodak film tank

Photo or Illustration Based: photo

Color or Black and White: bw

Description / Notes: Photo of a woman at a window holding a

roll of film. The Tank is on the table.

Binder: 1909-1913 Ad Books 6 of 6

Year: Feb-13

Slogan: The Experience is in the Tank

Subject(s): woman

Text: Besides the pleasure, the convenience, the cleanliness and simplicity of developing the Kodak Film Tank way; there is--best of all--the satisfaction of securing better results. The clean, snappy, tank-developed negatives make the most perfect prints.

Implied Audience: women / general

Product: Kodak film tank

Photo or Illustration Based: photo

Color or Black and White: bw

Description / Notes: Photo of a woman at a window holding a roll of film. The Tank is on the table. Same image as above,

different text.

Binder: 1909-1913 Ad Books 6 of 6

Year: Jul-13

Slogan: Kodak Film Tank

Subject(s): Woman

Text: Make the most of vacation pleasures--develop your films on the spot with a Kodak Film Tank. Not fuss or bother--gives better quality in your negatives--adds zest to the good times. The

Experience is in the tank

Implied Audience: women / genera
Product: Kodak film tank

Photo or Illustration Based: photo

Color or Black and White: bw

Description / Notes: Photo of a woman at a window, holding up a film roll. Tank is on the table.

Binder: 1909-1913 Ad Books 6 of 6

Year: Jul-13

Slogan: Kodak Film Tank

Subject(s): woman

Text: Be Certain of your results and enjoy as well the convenience

and pleasure of developing anywhere, all by daylight, with a Kodak

Film Tank.

Implied Audience: women / general

Product: Kodak film tank

Photo or Illustration Based: photo

Color or Black and White: bw

Description / Notes: Photo of a woman at a window, holding up a film roll. Tank is on the table. Same image as above, with different text.

Binder: 1909-1913 Ad Books 6 of 6

Year: Jul-13

Slogan: Kodak Film Tank

Subject(s): Woman

Text: The best of every exposure in every roll of film is assured

when you use a Kodak Film Tank. The principle is right, the chemicals are correct, the results are certain.

Implied Audience: women / general

Product: Kodak film tank

Photo or Illustration Based: photo

Color or Black and White: bw

Description / Notes: Photo of a woman at a window, holding up

a film roll. Tank is on the table. Same image as above, with

different text.

Binder: 1909-1913 Ad Books 6 of 6

Year: 1913

Slogan: Little Helps / Has to Be 
Subject(s): product - film \& paper

Product: Kodak portrait attachment / Eastman N.C. Film

Description / Notes: Article.

Binder: 1909-1913 Ad Books 6 of 6

Year: 1913

Slogan: The Spirit of Summer-time

Subject(s): product - film \& paper

Product: Kodak Velvet Green Paper

Color or Black and White:

Binder: 1909-1913 Ad Books 6 of 6

Year: Apr-13

Slogan: Make a few prints from your first spring negatives on

Kodak Velvet Green Paper

Subject(s): product - film \& paper

Product: Kodak Velvet Green Paper

Color or Black and White:

Binder: 1909-1913 Ad Books 6 of 6

Year: May-13

Slogan: Add the necessary touch of realism to the landscape by

making the print on Kodak Velvet Green Paper

Subject(s): product - film \& paper

Product: Kodak Velvet Green Paper

Color or Black and White:

Binder: 1909-1913 Ad Books 6 of 6

Year: Oct-13

Slogan: Get as close to the tones of nature as possible. Make prints of the summer landscape on Kodak Velvet Green

Subject(s): product - film \& paper

Product: Kodak Velvet Green Paper

Color or Black and White:

Binder: 1909-1913 Ad Books 6 of 6

Year: May-13

Slogan: The Kodiopticon

Subject(s): product - misc.
Product: Kodiopticon

Description / Notes: Lantern Slide projector. Complete with

Mazda lamp and carrying case price: $\$ 20.00$

Binder: 1909-1913 Ad Books 6 of 6

Year: Jul-13

Slogan: Kodak Advertising Competition 3,000.00 in Cash Prizes Subject(s): product - misc.

Product: Photo contest

Description / Notes: "For pictures to be used in illustrating Kodak Advertisements"

Binder: 1909-1913 Ad Books 6 of 6

Year: Sep-13

Slogan: Sixty Days Left Kodak Advertising Competition

Subject(s): product - misc.

Product: Photo contest

Description / Notes: Contest closes November 1st 1913. \$

3000.00 in prizes

Binder: 1909-1913 Ad Books 6 of 6

Year: Oct-13

Slogan: Thirty Days Left Kodak Advertising Collection

Subject(s): product - misc.

Product: Photo contest

Description / Notes: Contest closes November 1st 1913. \$

3000.00 in prizes

Binder: 1909-1913 Ad Books 6 of 6

Year: Sep-13

Slogan: Make sure that every print you deliver is safe, using a

Rounds Print Washer

Subject(s): product - darkroom

Product: Rounds Print Washer

Color or Black and White:

Binder: 1909-1913 Ad Books 6 of 6

Year: 1913

Slogan: Seed Plates The Greatest Efficiency. 
Subject(s): product - film \& paper Product: Seed Dry Plates

Color or Black and White:

Binder: 1909-1913 Ad Books 6 of 6 Year: Apr-13

Slogan: Uniformly Reliable

Subject(s): product - film \& paper

Product: Seed Dry Plates

Color or Black and White:

Binder: 1909-1913 Ad Books 6 of 6

Year: May-13

Slogan: Uniformly Dependable Speed and Quality

Subject(s): product - film \& paper

Product: Seed Dry Plates

Color or Black and White:

Binder: 1909-1913 Ad Books 6 of 6

Year: May-13

Slogan: Quality in Fast Plates

Subject(s): product - film \& paper

Product: Seed Dry Plates

Color or Black and White:

Binder: 1909-1913 Ad Books 6 of 6

Year: Jun-13

Slogan: The superior quality of Seed Dry Plates is invariable

Subject(s): product - film \& paper

Product: Seed Dry Plates

Color or Black and White:

Binder: 1909-1913 Ad Books 6 of 6

Year: Jul-13

Slogan: Work for Gradation

Subject(s): product - film \& paper

Product: Seed Dry Plates

Color or Black and White:
Binder: 1909-1913 Ad Books 6 of 6

Year: Aug-13

Slogan: It's a Seed Plate you need.

Subject(s): product - film \& paper

Product: Seed Dry Plates

Color or Black and White:

Binder: 1909-1913 Ad Books 6 of 6

Year: Sep-13

Slogan: Seed Capabilities

Subject(s): product - film \& paper

Product: Seed Dry Plates

Color or Black and White:

Binder: 1909-1913 Ad Books 6 of 6

Year: Oct-13

Slogan: Seed Results

Subject(s): product - film \& paper

Product: Seed Dry Plates

Color or Black and White:

Binder: 1909-1913 Ad Books 6 of 6

Year: Apr-13

Slogan: Works Right--Keeps Right Under the most unfavorable circumstances

Subject(s): product - film \& paper

Product: Standard Dry Plate

Color or Black and White:

Binder: 1909-1913 Ad Books 6 of 6

Year: Oct-13

Slogan: The Eastman Timer / Thermometer Stirring Rod

Subject(s): product - darkroom

Product: The Eastman Timer / Thermometer Stirring Rod

Description / Notes: Price for timer: $\$ 2.00$ Price for

thermometer stirring rod: $\$ 0.60$

Binder: 1909-1913 Ad Books 6 of 6

Year: Feb-13 
Slogan: Tozol The Simplified Developing Agent for Photographic Papers

Subject(s): product - darkroom

Product: Tozol Developing Agent

Color or Black and White:

Binder: 1909-1913 Ad Books 6 of 6

Year: Jul-13

Slogan: You don't have to make a certain kind of negative to fit Velox

Subject(s): product - film \& paper

Product: Velox

Color or Black and White:

Binder: 1909-1913 Ad Books 6 of 6

Year: Aug-13

Slogan: Double Printing

Subject(s): product - film \& paper

Product: Velox

Color or Black and White:

Binder: 1909-1913 Ad Books 6 of 6

Year: Sep-13

Slogan: The Experienced Amateur uses Velox

Subject(s): product - film \& paper

Product: Velox

Color or Black and White:

Binder: 1909-1913 Ad Books 6 of 6

Year: 1913

Slogan: Velox was and is the First

Subject(s): product - film \& paper

Product: Velox paper

Color or Black and White:

Binder: 1909-1913 Ad Books 6 of 6

Year: 1913

Slogan: The making of a best possible print is in every sheet of Velox
Subject(s): product - film \& paper

Product: Velox paper

Color or Black and White:

Binder: 1909-1913 Ad Books 6 of 6

Year: Mar-13

Slogan: Cheaper papers have but one thing to recommend them-their cheapness.

Subject(s): product - film \& paper

Product: Velox paper

Color or Black and White:

Binder: 1909-1913 Ad Books 6 of 6

Year: Mar-13

Slogan: The Paper that fits.

Subject(s): product - film \& paper

Product: Velox paper

Color or Black and White:

Binder: 1909-1913 Ad Books 6 of 6

Year: May-13

Slogan: To think of quality is to think of Velox

Subject(s): product - film \& paper

Product: Velox paper

Color or Black and White:

Binder: 1909-1913 Ad Books 6 of 6

Year: May-13

Slogan: If you have not used Velox

Subject(s): product - film \& paper

Product: Velox paper

Color or Black and White:

Binder: 1909-1913 Ad Books 6 of 6 Year: Jul-13

Slogan: Make Velox Your Paper

Subject(s): product - film \& paper

Product: Velox paper

Color or Black and White: 
Binder: 1909-1913 Ad Books 6 of 6

Year: Oct-13

Slogan: Begin right, use Velox

Subject(s): product - film \& paper

Product: Velox paper

Color or Black and White:

Binder: 1909-1913 Ad Books 6 of 6

Year: Jul-13

Slogan: The Vest Pocket Kodak Special

Subject(s): product - camera

Product: Vest Pocket Kodak Special

Description / Notes: Price: $\$ 25.00$

Binder: 1909-1913 Ad Books 6 of 6

Year: 1913

Slogan: Show your individuality

Subject(s): product - film \& paper

Product: Zelta Paper

Color or Black and White:

Binder: 1909-1913 Ad Books 6 of 6

Year: Feb-13

Slogan: It's your individuality that counts

Subject(s): product - film \& paper

Product: Zelta Paper

Color or Black and White:

Binder: 1909-1913 Ad Books 6 of 6

Year: Apr-13

Slogan: Character in Print

Subject(s): product - film \& paper

Product: Zelta Paper

Color or Black and White:

Binder: 1909-1913 Ad Books 6 of 6

Year: May-13

Slogan: Stamp your work with the mark of distinctive quality
Subject(s): product - film \& paper

Product: Zelta Paper

Color or Black and White:

Binder: $1913-19171$ of 4

Slogan: The Kodak Film Tank

Subject(s): Kodak Girl

Text: Makes developing a most simple, all-by-daylight process,

But of greater importance than its simplicity of convenience - the best results are assured from every exposure. Tank developed negatives are free from fog, scratches and finger marks - have the snap and brilliancy that makes good prints.

Implied Audience: women / general

Product: Film tank

Photo or Illustration Based: photo

Color or Black and White: bw

Description / Notes: Photo of a woman looking at negatives at a window.

Binder: $1913-19171$ of 4

Slogan: Kodak Film Tank

Subject(s): Kodak Girl

Text: On every vacation take a Kodak Film Tank. Half the pleasure of vacation picture making is in developing your films on the spot, seeing your results, making your picture story complete. And more then half the pleasure of developing in the Kodak film tank is in the satisfaction of securing better results. The experience is in the tank.

Implied Audience: women / general

Product: Film tank

Photo or Illustration Based: photo

Color or Black and White: bw

Description / Notes: Photograph of a woman in the window looking at negatives.

Binder: $1913-19171$ of 4

Slogan: The Kodak Film Tank

Subject(s): Kodak Girl

Text: Development Simplified. The Kodak film tank makes 
developing a part of the pleasure of picture making-insures the best result from every exposure - offers the convenience of developing anywhere without a darkroom. The film tank takes care of the greatest variations in exposure, yields clean, crisp negatives of a printing quality not possible to obtain by hand development.

Implied Audience: women / general

Product: Film tank

Photo or Illustration Based: photo

Color or Black and White: bw

Description / Notes: Photo of a woman looking at negatives at a window.

Binder: $1913-19171$ of 4

Slogan: Kodak Film Tank

Subject(s): Kodak Girl

Text: Include a Kodak Film Tank in your vacation kit. Develop your films on the spot - see your results before you leave the scene of your good times. The film tank assures the best results takes care of any reasonable variations in the time of your

exposure- renders clean, crisp, snappy negatives. And all without the fuss of a darkroom.

Implied Audience: women / general

Product: Film tank

Photo or Illustration Based: photo

Color or Black and White: bw

Description / Notes: Photo is a woman developing negatives at the window.

Binder: 1913-1917 1 of 4

Slogan: Make the Most of the home side of photography Subject(s): child

Text: The charm of home surroundings offers a wealth of material for picture making. And the Kodak portrait attachment brings the entire home within reach of your Kodak lens. Slip the Portrait Attachment over the Kodak lens and you are ready for portraits of other close work. With fixed focus Brownies and Kodaks it brings objects in focus at exactly 3.5 feet - with focusing Kodaks, one may work as close as 2 feet 8 inches.

Implied Audience: families
Product: portrait attachment

Photo or Illustration Based: photo

Color or Black and White: bw

Description / Notes: Photo of a child captioned "Made with a Brownie and portrait attachment.

Binder: $1913-19171$ of 4

Slogan: Kodak Portrait Attachment

Subject(s): woman

Text: Place your camera close to your subject, then bring the lens into focus by using the Kodak Portrait Attachment ... It may be readily seen that with the Kodak Portrait Attachment, thousands of small objects become interesting subjects for your Kodak.

Product: portrait attachment

Photo or Illustration Based: photo

Color or Black and White: bw

Description / Notes: Photo of a woman with flowers caption:

"Made with Kodak and Kodak Portrait Attachment."

Binder: $1913-19172$ of 4

Year: Dec-14

Slogan: The Autographic Kodak

Subject(s): product - camera

Text: The autographic negative furnishes you permanent and positive identification. Date and title your negatives at the time you make them. There can then be no doubt in the years to come as to when, where to under what conditions such and such a picture was taken. ...Facts concerning the children and their age, when the picture was taken, interesting places you visit, a friend's autograph below his portrait, the stop, exposure, and date - the possibilities of the autographic record are endless.

Product: autographic Kodak

Photo or Illustration Based: photo

Color or Black and White: bw

Description / Notes: caption for photograph: Negative with autographic record.

Binder: $1913-19172$ of 4

Year: Nov-14 
Slogan: Yuletide Calendars

Subject(s): product - misc

Text: It's time to think of holiday remembrances. Solve the

problem by mounting your prints in Yuletide calendars. They cost but a trifle - make most appropriate gifts. The calendars are made

for all standard size amateur prints with vertical or horizontal

openings, have easel backs and silk cord...

Product: yuletide calendars

Photo or Illustration Based: photo

Color or Black and White: bw

Description / Notes: ?

Binder: 1913-1917 Amateur Ads 1 of 4

Year: Apr-14

Slogan: You can make better pictures by knowing first, "How to Make Good Pictures"

Subject(s): product - misc.

Product: "How to Make Good Pictures" booklet

Description / Notes: Booklet price: $\$ 0.25$. Library Edition, cloth covers: $\$ 1.00$

Binder: 1913-1917 Amateur Ads 1 of 4

Year: May-14

Slogan: A help to better results

Subject(s): product - misc.

Product: "How to Make Good Pictures" booklet

Description / Notes: Article.

Binder: 1913-1917 Amateur Ads 1 of 4

Year: Mar-14

Slogan: If it's a matter of quality, use Artura

Subject(s): product - film \& paper

Product: Artura

Color or Black and White:

Binder: 1913-1917 Amateur Ads 1 of 4

Year: May-14

Slogan: For the Large print, make Artura Carbon Black

Subject(s): product - film \& paper
Product: Artura Carbon Black

Color or Black and White:

Binder: 1913-1917 Amateur Ads 1 of 4

Year: 1914

Slogan: Establish the highest standard of quality with Artura

Subject(s): product - film \& paper

Product: Artura paper

Color or Black and White:

Binder: 1913-1917 Amateur Ads 1 of 4

Year: Dec-13

Slogan: Artura A paper without a disappointment

Subject(s): product - film \& paper

Product: Artura paper

Color or Black and White:

Binder: 1913-1917 Amateur Ads 1 of 4

Year: Jan-14

Slogan: Artura, There's not a disappointment in it.

Subject(s): product - film \& paper

Product: Artura paper

Color or Black and White:

Binder: 1913-1917 Amateur Ads 1 of 4

Year: Apr-14

Slogan: If you want every result to count, use Artura

Subject(s): product - film \& paper

Product: Artura Paper

Color or Black and White

Binder: 1913-1917 Amateur Ads 1 of 4

Year: May-14

Slogan: The Real success won and maintained on quality alone

Subject(s): product - film \& paper

Product: Artura Paper

Color or Black and White:

Binder: 1913-1917 Amateur Ads 1 of 4 
Year: Aug-14

Slogan: For the best results continuously, use Artura

Subject(s): product - film \& paper

Product: Artura Paper

Color or Black and White:

Binder: 1913-1917 Amateur Ads 1 of 4

Year: Sep-14

Slogan: Artura The Paper without a disappointment

Subject(s): product - film \& paper

Product: Artura Paper

Color or Black and White:

Binder: 1913-1917 Amateur Ads 1 of 4

Year: Aug-14

Slogan: The Autographic Kodaks

Subject(s): product - camera

Product: Autographic Kodak

Color or Black and White:

Binder: 1913-1917 Amateur Ads 1 of 4

Year: Mar-14

Slogan: Brownie Enlarging Camera

Subject(s): product - camera

Product: Brownie Enlarging Camera

Description / Notes: No. 2 price: $\$ 2.00$. No. 3 price: $\$ 3.00$. No.

4 Price: $\$ 4.00$

Binder: 1913-1917 Amateur Ads 1 of 4

Year: Jul-14

Slogan: The Distinctive character of the print on Eastman EB or ES Platinum

Subject(s): product - film \& paper

Product: Eastman EB and ES Platinum

Color or Black and White:

Binder: 1913-1917 Amateur Ads 1 of 4

Year: Dec-13

Slogan: Secure perfect control of your illumination with Eastman
Flash Sheets

Subject(s): product - misc.

Product: Eastman Flash Sheets

Color or Black and White:

Binder: 1913-1917 Amateur Ads 1 of 4

Year: May-14

Slogan: Eastman Four-in-one Groupers

Subject(s): product - misc.

Product: Eastman Four-in-one Groupers

Description / Notes: Price for a set: $\$ 10.00$ Solid Oak

Binder: 1913-1917 Amateur Ads 1 of 4

Year: Jan-14

Slogan: Make Sure Your Chemicals Fit

Subject(s): product - Chemicals

Product: Eastman Kodak Company Tested Chemicals

Color or Black and White:

Binder: 1913-1917 Amateur Ads 1 of 4

Year: May-14

Slogan: It costs no more to be certain; use Kodak Acid Fixing

Powder

Subject(s): product - Chemicals

Product: Eastman Kodak Company Tested Chemicals

Description / Notes: Kodak acid fixing powder 1lb: \$0.25. 1/2

pound price: $\$ 0.15 .1 / 4$ pound price: $\$ 0.10$

Binder: 1913-1917 Amateur Ads 1 of 4

Year: Jul-14

Slogan: The perfect developing agent, Elon

Subject(s): product - Chemicals

Product: Eastman Kodak Company Tested Chemicals

Color or Black and White:

Binder: 1913-1917 Amateur Ads 1 of 4

Year: Jul-14

Slogan: Buy the right chemicals

Subject(s): product - Chemicals 
Product: Eastman Kodak Company Tested Chemicals Color or Black and White:

Binder: 1913-1917 Amateur Ads 1 of 4

Year: Aug-14

Slogan: Insist on Eastman Tested Chemicals, in the container which bears this seal.

Subject(s): product - Chemicals

Product: Eastman Kodak Company Tested Chemicals

Color or Black and White:

Binder: 1913-1917 Amateur Ads 1 of 4

Year: Aug-14

Slogan: Kodak Acid Fixing Powder

Subject(s): product - Chemicals

Product: Eastman Kodak Company Tested Chemicals

Color or Black and White:

Binder: 1913-1917 Amateur Ads 1 of 4

Year: Jan-14

Slogan: Quality plus Convenience Eastman Portrait Films

Subject(s): product - film \& paper

Product: Eastman Portrait Films

Color or Black and White:

Binder: 1913-1917 Amateur Ads 1 of 4

Year: Mar-14

Slogan: Halation is done away with--the cause removed, in

Eastman Portrait Films

Subject(s): product - film \& paper

Product: Eastman Portrait Films

Color or Black and White:

Binder: 1913-1917 Amateur Ads 1 of 4

Year: Aug-14

Slogan: A higher standard of negative quality is made possible by

Eastman Portrait Films

Subject(s): product - film \& paper

Product: Eastman Portrait Films

\section{Color or Black and White:}

Binder: 1913-1917 Amateur Ads 1 of 4 Year: 1914

Slogan: The Eastman Studio Reflector

Subject(s): product - misc.

Product: Eastman Studio Reflector

Color or Black and White:

Binder: 1913-1917 Amateur Ads 1 of 4

Year: Mar-14

Slogan: Watch the Temperature / Is your camera far sighted?

Subject(s): product - film \& paper

Product: Eastman thermometer, Kodak portrait attachment

Color or Black and White:

Binder: 1913-1917 Amateur Ads 1 of 4

Year: Jul-14

Slogan: Eastman View Cameras, No. 1 and No. 2

Subject(s): product - camera

Product: Eastman View Cameras

Description / Notes: Price ranges from $\$ 19.00$ to $\$ 72.00$

Binder: 1913-1917 Amateur Ads 1 of 4

Year: Sep-14

Slogan: V.P.K. Enlarging or a Brownie Enlarging Camera

Subject(s): product - camera

Product: Enlarging Cameras

Description / Notes: Price from $\$ 1.75$ to $\$ 4.00$

Binder: 1913-1917 Amateur Ads 1 of 4

Year: May-14

Slogan: Kodak Autotime Scale

Subject(s): product - camera

Product: Kodak Autotime Scale

Description / Notes: Price for compound shutter: \$1.50. Price

for all other shutters: $\$ 1.00$

Binder: 1913-1917 Amateur Ads 1 of 4 
Year: 1914

Slogan: Snow

Subject(s): product - camera

Product: Kodak cameras

Description / Notes: Article.

Binder: 1913-1917 Amateur Ads 1 of 4

Year: Dec-13

Slogan: A Kodak Party

Subject(s): product - camera

Product: Kodak cameras

Description / Notes: Article.

Binder: 1913-1917 Amateur Ads 1 of 4

Year: Apr-14

Slogan: Anticipation

Subject(s): product - camera

Product: Kodak cameras

Description / Notes: Article.

Binder: 1913-1917 Amateur Ads 1 of 4

Year: Dec-13

Slogan: Many of your Kodak negatives would be truer to nature if

made with a Kodak Color Screen

Subject(s): product - camera

Product: Kodak Color Screen

Description / Notes: Price: $\$ 0.50, \$ 0.75, \$ 1.00$

Binder: 1913-1917 Amateur Ads 1 of 4

Year: May-14

Slogan: Avoid blank white skies in your prints, use a Kodak Color Screen

Subject(s): product - camera

Product: Kodak Color Screen

Description / Notes: Price: $\$ 0.50, \$ 0.75, \$ 1.00$

Binder: 1913-1917 Amateur Ads 1 of 4

Year: Jan-14

Slogan: The Kodak Correspondence College
Subject(s): product - misc.

Product: Kodak correspondence college

Description / Notes: Article.

Binder: 1913-1917 Amateur Ads 1 of 4

Year: Dec-13

Slogan: Holiday Remembrances / Home Photography

Subject(s): product - film \& paper

Product: Kodak film and paper / Portrait attachment

Description / Notes: Article.

Binder: 1913-1917 Amateur Ads 1 of 4

Year: Dec-13

Slogan: A Dark-room or The Kodak Film Tank

Subject(s): product - darkroom

Product: Kodak Film Tank

Color or Black and White:

Binder: 1913-1917 Amateur Ads 1 of 4

Year: Jan-14

Slogan: The Kodak Film Tank

Subject(s): product - darkroom

Product: Kodak film tank

Color or Black and White:

Binder: 1913-1917 Amateur Ads 1 of 4

Year: Mar-14

Slogan: There's the element of certainty in the work of the Kodak Film Tank

Subject(s): product - darkroom

Product: Kodak Film Tank

Color or Black and White:

Binder: 1913-1917 Amateur Ads 1 of 4

Year: Apr-14

Slogan: The Satisfaction of developing your films in the Kodak

Film Tank

Subject(s): product - darkroom

Product: Kodak Film Tank 


\section{Color or Black and White:}

Binder: 1913-1917 Amateur Ads 1 of 4

Year: May-14

Slogan: Develop your films in the Kodak Film Tank

Subject(s): product - darkroom

Product: Kodak film tank

Color or Black and White:

Binder: 1913-1917 Amateur Ads 1 of 4

Year: May-14

Slogan: The Kodak Magnesium Ribbon Holder

Subject(s): product - darkroom

Product: Kodak Magnesium Ribbon Holder

Description / Notes: Price: $\$ 0.20$. Kodak alcohol lamp for

burning ribbon: $\$ 0.20$

Binder: 1913-1917 Amateur Ads 1 of 4

Year: May-14

Slogan: For all exposures over 1/25 of a second, use a Kodak

Metal Tripod

Subject(s): product - misc.

Product: Kodak Metal tripod

Description / Notes: No. 0 price: $\$ 1.60$. No. 1 price: $\$ 2.50$. No.

2 price: $\$ 3.25$. Leatherette case for No. 0: $\$ 0.75$. Leather Carrying

case: $\$ 1.50$

Binder: 1913-1917 Amateur Ads 1 of 4

Year: 1914

Slogan: The Big Camera Possibilities of a Kodak are found in the Kodak Portrait Attachment

Subject(s): product - camera

Product: Kodak portrait attachment

Description / Notes: Price: $\$ 0.50$

Binder: 1913-1917 Amateur Ads 1 of 4

Year: Apr-14

Slogan: Get close to nature with a Kodak portrait attachment

Subject(s): product - camera
Product: Kodak portrait attachment

Description / Notes: Price: $\$ 0.50$

Binder: 1913-1917 Amateur Ads 1 of 4

Year: Apr-14

Slogan: The Kodak Portrait Attachment

Subject(s): product - camera

Product: Kodak portrait attachment

Description / Notes: Price: $\$ 0.50$

Binder: 1913-1917 Amateur Ads 1 of 4

Year: Aug-14

Slogan: Kodak portrait attachment

Subject(s): product - camera

Product: Kodak portrait attachment

Description / Notes: Price: $\$ 0.50$

Binder: 1913-1917 Amateur Ads 1 of 4

Year: Jun-14

Slogan: When you pack for your vacation trip

Subject(s): product - misc.

Product: Kodak System

Description / Notes: Article.

Binder: 1913-1917 Amateur Ads 1 of 4

Year: Sep-14

Slogan: Kodak Trimming Boards

Subject(s): product - misc.

Product: Kodak trimming boards

Color or Black and White:

Binder: 1913-1917 Amateur Ads 1 of 4

Year: Apr-14

Slogan: Make prints from your choice negatives on Kodak Velvet Green Paper

Subject(s): product - film \& paper

Product: Kodak Velvet Green Paper

Color or Black and White: 
Binder: 1913-1917 Amateur Ads 1 of 4

Year: May-14

Slogan: Print your vacation postals by daylight on Kodak Velvet Green paper

Subject(s): product - film \& paper

Product: Kodak Velvet Green Paper

Color or Black and White:

Binder: 1913-1917 Amateur Ads 1 of 4

Year: May-14

Slogan: The Developer that fits--Nepera Solution

Subject(s): product - Chemicals

Product: Nepera Solution

Description / Notes: Price 4oz bottle: $\$ 0.20 .160 z$ bottle. $\$ 0.60$.

Eastman M.Q. Tubes: $\$ 0.05$.

Binder: 1913-1917 Amateur Ads 1 of 4

Year: Aug-14

Slogan: No. 4 Majestic Print Dryer

Subject(s): product - darkroom

Product: No. 4 Majestic Print Dryer

Description / Notes: Price: $\$ 45.00$

Binder: 1913-1917 Amateur Ads 1 of 4

Year: May-14

Slogan: Great efficiency at small cost. The R.O.C. Post Card

Printer

Subject(s): product - film \& paper

Product: R.O.C. Post Card Printer

Description / Notes: price: $\$ 7.50$

Binder: 1913-1917 Amateur Ads 1 of 4

Year: Dec-13

Slogan: In the busy holiday season, make sure your prints are safe--use a Rounds Print Washer.

Subject(s): product - darkroom

Product: Rounds Print Washer

Description / Notes: 100 capacity price: $\$ 10.00 .200$ capacity price: $\$ 20.00$
Binder: 1913-1917 Amateur Ads 1 of 4

Year: May-14

Slogan: The Wratten Safelight Lamp

Subject(s): product - misc.

Product: Safelight Lamp

Description / Notes: Price for No. 1: \$9.00. No. 2: $\$ 6.00$. Series 1 safelight: $\$ 1.00$. Series 2 safelight: $\$ 1.00$. Series 3 safelight:

$\$ 1.00$

Binder: 1913-1917 Amateur Ads 1 of 4

Year: Dec-13

Slogan: You are always sure the plate is right when you use a Seed

Subject(s): product - film \& paper

Product: Seed Dry Plates

Color or Black and White:

Binder: 1913-1917 Amateur Ads 1 of 4

Year: Jan-14

Slogan: The right plate for Dark Days

Subject(s): product - film \& paper

Product: Seed Dry Plates

Color or Black and White:

Binder: 1913-1917 Amateur Ads 1 of 4

Year: Mar-14

Slogan: Uniform Dependability

Subject(s): product - film \& paper

Product: Seed Dry Plates

Color or Black and White:

Binder: 1913-1917 Amateur Ads 1 of 4

Year: Jul-14

Slogan: Seed Plates. The ideal plate for portraiture.

Subject(s): product - film \& paper

Product: Seed Dry Plates

Color or Black and White: 
Binder: 1913-1917 Amateur Ads 1 of 4

Year: 1914

Slogan: The best amateurs and finishers of amateur work use Velox

Subject(s): product - film \& paper

Product: Velox

Color or Black and White:

Binder: 1913-1917 Amateur Ads 1 of 4

Year: Sep-14

Slogan: For your vacation pictures--Velox

Subject(s): product - film \& paper

Product: Velox

Color or Black and White:

Binder: 1913-1917 Amateur Ads 1 of 4

Year: Dec-13

Slogan: Velox Lantern Slide Film

Subject(s): product - film \& paper

Product: Velox lantern slide film

Description / Notes: Price Velox Lantern Slide Film per Doz.

$\$ 0.30$. Kodiopticon complete with Mazda lamp $\$ 20.00$.

Binder: 1913-1917 Amateur Ads 1 of 4

Year: 1913

Slogan: Get the best results--make the print on Velox

Subject(s): product - film \& paper

Product: Velox paper

Color or Black and White:

Binder: 1913-1917 Amateur Ads 1 of 4

Year: 1914

Slogan: Profit by the successful experience of those who use Velox

Subject(s): product - film \& paper

Product: Velox paper

Color or Black and White:

Binder: 1913-1917 Amateur Ads 1 of 4
Year: Dec-13

Slogan: For the good of all your negatives, insist on Velox

Subject(s): product - film \& paper

Product: Velox paper

Color or Black and White:

Binder: 1913-1917 Amateur Ads 1 of 4

Year: Dec-13

Slogan: Velox the paper that's quite right

Subject(s): product - film \& paper

Product: Velox paper

Color or Black and White:

Binder: 1913-1917 Amateur Ads 1 of 4

Year: Mar-14

Slogan: Select the surface of paper most suitable to your negative--then make the print on Velox

Subject(s): product - film \& paper

Product: Velox paper

Color or Black and White:

Binder: 1913-1917 Amateur Ads 1 of 4

Year: Apr-14

Slogan: To make the most of every negative, make the print on Velox

Subject(s): product - film \& paper

Product: Velox paper

Color or Black and White:

Binder: 1913-1917 Amateur Ads 1 of 4

Year: May-14

Slogan: First of all, select Velox

Subject(s): product - film \& paper

Product: Velox paper

Color or Black and White:

Binder: 1913-1917 Amateur Ads 1 of 4

Year: Jul-14

Slogan: The amateur's own paper Velox 
Subject(s): product - film \& paper

Product: Velox paper

Color or Black and White:

Binder: 1913-1917 Amateur Ads 1 of 4

Year: Aug-14

Slogan: For Results--Velox

Subject(s): product - film \& paper

Product: Velox paper

Color or Black and White:

Binder: 1913-1917 Amateur Ads 1 of 4

Year: 1913

Slogan: Velox Transparent Water Color Stamps

Subject(s): product - misc.

Product: Velox Transparent Water Color Stamps

Description / Notes: Booklet of 12 colors price: $\$ 0.25$. Separate colors, 2 leaves: $\$ 0.05$. Set of three camel hair brushes: $\$ 0.50$. Velox Water Color Palette: $\$ 0.25$. Complete Velox water color outfit: $\$ 0.75$

Binder: 1913-1917 Amateur Ads 1 of 4

Year: May-14

Slogan: Color your prints with Velox Transparent Water Color

Stamps

Subject(s): product - misc.

Product: Velox Transparent Water Color Stamps

Color or Black and White:

Binder: 1913-1917 Amateur Ads 1 of 4

Year: Sep-14

Slogan: When vacation days are over--color your prints with Velox Transparent Water Color Stamps

Subject(s): product - misc.

Product: Velox Transparent Water Color Stamps

Description / Notes: Price for complete set: $\$ 0.75$

Binder: 1913-1917 Amateur Ads 1 of 4

Year: Jul-14
Slogan: The Vest Pocket Kodak with Fixed Focus Anastigmat Lens Subject(s): product - camera

Product: Vest Pocket Kodak

Description / Notes: Article.

Binder: 1913-1917 Amateur Ads 1 of 4

Year: Aug-14

Slogan: The Essentials of Efficiency.

Subject(s): product - camera

Product: Vest Pocket Kodak

Description / Notes: Article.

Binder: 1913-1917 Amateur Ads 1 of 4

Year: Aug-14

Slogan: V.P. Kodak Enlarging Camera

Subject(s): product - film \& paper

Product: Vest Pocket Kodak Enlarging Camera

Description / Notes: Price from $\$ 1.75$ to $\$ 4.00$

Binder: 1913-1917 Amateur Ads 2 of 4

Year: Nov-14

Slogan: How to Make Good Pictures

Subject(s): product - misc.

Product: "How to Make Good Pictures" booklet

Description / Notes: Price for booklet: \$0.25 Price for library

edition: $\$ 1.00$

Binder: 1913-1917 Amateur Ads 2 of 4

Year: Jun-15

Slogan: Anastigmatic and Autographic \$27.50 The New 3a Kodak

Subject(s): product - camera

Product: 3a Kodak

Description / Notes: Price for Kodak with Anastigmat lens:

$\$ 27.50$. Price with Rapid Rectilinear Lens: $\$ 22.50$

Binder: 1913-1917 Amateur Ads 2 of 4

Year: Mar-15

Slogan: The new brand of Artura paper: Artura Aegis

Subject(s): product - film \& paper 
Product: Artura Aegis Paper

Color or Black and White:

Binder: 1913-1917 Amateur Ads 2 of 4

Year: May-15

Slogan: Make Sepia prints of quality with uniformity on: Artura

Aegis

Subject(s): product - film \& paper

Product: Artura Aegis Paper

Color or Black and White:

Binder: 1913-1917 Amateur Ads 2 of 4

Year: Jan-15

Slogan: Large Prints from small negatives, when made on Artura

Carbon Black

Subject(s): product - film \& paper

Product: Artura Carbon Black

Color or Black and White:

Binder: 1913-1917 Amateur Ads 2 of 4

Year: Nov-14

Slogan: The print tells the whole story of quality if made on Artura

Subject(s): product - film \& paper

Product: Artura paper

Color or Black and White:

Binder: 1913-1917 Amateur Ads 2 of 4

Year: Dec-14

Slogan: Build your success on quality--make your prints on Artura

Subject(s): product - film \& paper

Product: Artura Paper

Color or Black and White:

Binder: 1913-1917 Amateur Ads 2 of 4

Year: Feb-15

Slogan: Artura. The real success because it is based on superiority

Subject(s): product - film \& paper

Product: Artura Paper

Color or Black and White:
Binder: 1913-1917 Amateur Ads 2 of 4

Year: Oct-14

Slogan: Make your Kodak Autographic

Subject(s): product - camera

Product: Autographic Backs for Kodaks

Description / Notes: Price ranges from $\$ 3.50$ to $\$ 4.25$

Binder: 1913-1917 Amateur Ads 2 of 4

Year: Oct-14

Slogan: A distinctive Kodak convenience. Make your Kodak

Autographic

Subject(s): product - camera

Product: Autographic Backs for Kodaks

Description / Notes: Article.

Binder: 1913-1917 Amateur Ads 2 of 4

Year: Nov-14

Slogan: Back Your Memory With an Autographic Record

Subject(s): product - camera

Product: Autographic Backs for Kodaks

Description / Notes: Article.

Binder: 1913-1917 Amateur Ads 2 of 4

Year: Sep-14

Slogan: The Autographic Kodak

Subject(s): product - camera

Product: Autographic Kodak

Description / Notes: Article.

Binder: 1913-1917 Amateur Ads 2 of 4

Year: Mar-15

Slogan: Write it on the film--at the time.

Subject(s): woman

Text: The Autographic feature, the biggest photographic advance in twenty years, enabling you to write valuable data in the margin below each negative, at the time you made the picture has bee incorporated in all the popular-sized Kodaks--the line of

Autographic Kodaks is now complete 
Implied Audience: women / general

Product: Autographic Kodak

Photo or Illustration Based: photo

Color or Black and White: bw

Description / Notes: Photo of a woman in a white hat writing on

the autographic Kodak

Binder: 1913-1917 Amateur Ads 2 of 4

Year: Dec-14

Slogan: Simplicity Itself

Subject(s): product - camera

Product: Brownie Enlarging Camera

Description / Notes: Price from $\$ 1.75$ to $\$ 4.00$

Binder: 1913-1917 Amateur Ads 2 of 4

Year: Feb-15

Slogan: Brownie Enlarging Camera. The larger print tells the same story but in bigger type.

Subject(s): product - camera

Product: Brownie Enlarging Camera

Description / Notes: Price: from $\$ 1.75$ to $\$ 4.00$

Binder: 1913-1917 Amateur Ads 2 of 4

Year: Mar-15

Slogan: No focusing; no dark room with the Brownie Enlarging

Camera

Subject(s): product - camera

Product: Brownie Enlarging Camera

Description / Notes: Price: from $\$ 1.75$ to $\$ 4.00$

Binder: 1913-1917 Amateur Ads 2 of 4

Year: Apr-15

Slogan: Enlarging with the Bother Left Out

Subject(s): product - camera

Product: Brownie Enlarging Camera

Description / Notes: Price from $\$ 1.75$ to $\$ 4.00$

Binder: 1913-1917 Amateur Ads 2 of 4

Year: Jan-15
Slogan: Do not depend on daylight if your house is equipped with electricity, The Brownie Enlarging Camera Illuminator

Subject(s): product - misc.

Product: Brownie Enlarging Camera Illuminator

Description / Notes: price: $\$ 3.00$

Binder: 1913-1917 Amateur Ads 2 of 4

Year: Jan-15

Slogan: Eastman Enlarging Outfit

Subject(s): product - camera

Product: Eastman Enlarging Outfit

Description / Notes: Complete price: $\$ 85.00$

Binder: 1913-1917 Amateur Ads 2 of 4

Year: Feb-15

Slogan: Eastman Film Clips

Subject(s): product - film \& paper

Product: Eastman Film Clips

Description / Notes: price for 3.5 inch pair: $\$ 0.25$. Price for 5

inch pair: $\$ 0.30$

Binder: 1913-1917 Amateur Ads 2 of 4

Year: Nov-14

Slogan: Eastman Flash Sheets

Subject(s): product - misc.

Product: Eastman Flash Sheets

Color or Black and White

Binder: 1913-1917 Amateur Ads 2 of 4

Year: Jan-15

Slogan: Every Evening has its picture possibilities. Eastman Flash

Sheets

Subject(s): product - misc

Product: Eastman Flash Sheets

Color or Black and White:

Binder: 1913-1917 Amateur Ads 2 of 4

Year: Mar-15

Slogan: The Hundred Per cent efficiency of Kodak / Ten Miles from 
a Tripod

Subject(s): product - misc.

Product: Eastman Flash Sheets / the Universal Clamp

Description / Notes: Article.

Binder: 1913-1917 Amateur Ads 2 of 4

Year: Oct-14

Slogan: Specify Eastman Permanent Crystal Pyro

Subject(s): product - Chemicals

Product: Eastman Kodak Company Tested Chemicals

Color or Black and White:

Binder: 1913-1917 Amateur Ads 2 of 4

Year: Dec-14

Slogan: It does for chemicals what certification does for a check.

Subject(s): product - Chemicals

Product: Eastman Kodak Company Tested Chemicals

Color or Black and White:

Binder: 1913-1917 Amateur Ads 2 of 4

Year: Jan-15

Slogan: Get the best results.

Subject(s): product - Chemicals

Product: Eastman Kodak Company Tested Chemicals

Color or Black and White:

Binder: 1913-1917 Amateur Ads 2 of 4

Year: Feb-15

Slogan: Our formulas specify Elon

Subject(s): product - Chemicals

Product: Eastman Kodak Company Tested Chemicals

Color or Black and White:

Binder: 1913-1917 Amateur Ads 2 of 4

Year: Apr-15

Slogan: The seal that makes you certain:

Subject(s): product - Chemicals

Product: Eastman Kodak Company Tested Chemicals

Color or Black and White:
Binder: 1913-1917 Amateur Ads 2 of 4

Year: May-15

Slogan: Specify E.K.Co. Tested Chemicals in your next order.

Subject(s): product - Chemicals

Product: Eastman Kodak Company Tested Chemicals

Color or Black and White:

Binder: 1913-1917 Amateur Ads 2 of 4

Year: Jun-15

Slogan: Look for this seal on the container

Subject(s): product - Chemicals

Product: Eastman Kodak Company Tested Chemicals

Color or Black and White:

Binder: 1913-1917 Amateur Ads 2 of 4

Year: May-15

Slogan: An ideal trimmer that will meet every professional requirement

Subject(s): product - misc.

Product: Eastman Paper Trimmer

Description / Notes: Price: from $\$ 5.00$ to $\$ 12.00$

Binder: 1913-1917 Amateur Ads 2 of 4

Year: Apr-15

Slogan: Eastman Photo Paste

Subject(s): product - misc

Product: Eastman Photo Paste

Description / Notes: Price: from $\$ 0.15$ to $\$ 0.25$

Binder: 1913-1917 Amateur Ads 2 of 4

Year: Nov-14

Slogan: Real Economy The Eastman Plate Tank

Subject(s): product - darkroom

Product: Eastman Plate Tank

Color or Black and White:

Binder: 1913-1917 Amateur Ads 2 of 4

Year: Jun-15 
Slogan: In hot weather. The Eastman Plate Tank

Subject(s): product - darkroom

Product: Eastman Plate Tank

Color or Black and White:

Binder: 1913-1917 Amateur Ads 2 of 4

Year: Apr-15

Slogan: From a photographer's letter regarding Eastman Portrait Film

Subject(s): product - film \& paper

Product: Eastman Portrait Film

Color or Black and White:

Binder: 1913-1917 Amateur Ads 2 of 4

Year: Jun-15

Slogan: Retain all the sparkle of highlights--all the delicacy of half-tones by using Eastman Portrait Films

Subject(s): product - film \& paper

Product: Eastman Portrait Films

Color or Black and White:

Binder: 1913-1917 Amateur Ads 2 of 4

Year: Feb-15

Slogan: A clean cut without a pull. The Eastman Rotary Print Trimmer

Subject(s): product - misc.

Product: Eastman Rotary Print Trimmer

Description / Notes: Price: \$5.00 Extra cutting discs: \$0.25

Binder: 1913-1917 Amateur Ads 2 of 4

Year: Nov-14

Slogan: A Universal Developer for Plates, Films and Paper.

Subject(s): product - Chemicals

Product: Eastman Special Developer

Description / Notes: Price: $\$ 0.25$

Binder: 1913-1917 Amateur Ads 2 of 4

Year: Dec-14

Slogan: The Eastman Studio Register System
Subject(s): product - misc.

Product: Eastman Studio Register System

Description / Notes: Price: $\$ 5.00$

Binder: 1913-1917 Amateur Ads 2 of 4

Year: May-15

Slogan: Accuracy and convenience combined in the Eastman

Studio Scale

Subject(s): product - darkroom

Product: Eastman Studio Scale

Description / Notes: Price: $\$ 3.00$

Binder: 1913-1917 Amateur Ads 2 of 4

Year: May-15

Slogan: Select the right outfit for commercial and out-door work.

Eastman View Camera No. 2.

Subject(s): product - camera

Product: Eastman View Camera No. 2.

Description / Notes: Price: from $\$ 25.00$ to $\$ 72.00$

Binder: 1913-1917 Amateur Ads 2 of 4

Year: Oct-14

Slogan: Enlarging Made Easy

Subject(s): product - camera

Product: Enlarging Cameras

Description / Notes: Price from $\$ 1.75$ to $\$ 4.00$

Binder: 1913-1917 Amateur Ads 2 of 4

Year: Jun-15

Slogan: With the new Kodak "Maskit" printing frame

Subject(s): product - film \& paper

Product: Kodak "Maskit" printing frame

Description / Notes: Price: from $\$ 0.40$ to $\$ 0.50$

Binder: 1913-1917 Amateur Ads 2 of 4

Year: Jul-15

Slogan: The Kodak Amateur Printer

Subject(s): product - darkroom

Product: Kodak Amateur Printer 
Description / Notes: Price: $\$ 5.00$

Binder: 1913-1917 Amateur Ads 2 of 4 Year: Feb-15

Slogan: It looks like paper but it sticks like glue

Subject(s): product - film \& paper

Product: Kodak Dry Mounting Tissue

Description / Notes: Price from $\$ 0.08$ to $\$ 1.50$

Binder: 1913-1917 Amateur Ads 2 of 4

Year: Mar-15

Slogan: The Kodak Film Tank

Subject(s): product - darkroom

Product: Kodak Film Tank

Color or Black and White:

Binder: 1913-1917 Amateur Ads 2 of 4

Year: May-15

Slogan: The Kodak Film Tank

Subject(s): product - darkroom

Product: Kodak film tank

Description / Notes: Article.

Binder: 1913-1917 Amateur Ads 2 of 4 Year: Jun-15

Slogan: With the Kodak Film Tank

Subject(s): product - darkroom

Product: Kodak film tank

Color or Black and White:

Binder: 1913-1917 Amateur Ads 2 of 4 Year: Jul-15

Slogan: Take Along a Kodak Film Tank

Subject(s): product - darkroom

Product: Kodak film tank

Color or Black and White:

Binder: 1913-1917 Amateur Ads 2 of 4 Year: Feb-15
Slogan: The Kodak Film Tank

Subject(s): woman

Text: The big link in the Kodak chain of daylight all the way. Aside from the convenience of the Kodak Film Tank but which you can develop your films anywhere, in broad daylight, the fact that tank development yields you the best possible negatives, in every case, is the one point that drives the story home.

Implied Audience: women / general

Product: Kodak film tank

Photo or Illustration Based: photo

Color or Black and White: bw

Description / Notes: Photo of a woman developing film by a window. Tank on the table.

Binder: 1913-1917 Amateur Ads 2 of 4

Year: Apr-15

Slogan: Kodak Film Tank

Subject(s): woman

Text: The Experience is in the Tank. The photographic amateur getting the results he should from each and every negative is using the Kodak film tank because it is only by tank development that best results are possible--not occasionally, but all the time.

Implied Audience: women / general

Product: Kodak film tank

Photo or Illustration Based: photo

Color or Black and White: bw

Description / Notes: Photo of a woman developing film by a window. Tank on the table. Photo is of a woman, but ad uses male pronouns.

Binder: 1913-1917 Amateur Ads 2 of 4

Year: Jan-15

Slogan: Kodak Anastigmat f.8.

Subject(s): product - camera

Product: Kodak lenses.

Description / Notes: Prices from $\$ 10.00$ to $\$ 25.00$

Binder: 1913-1917 Amateur Ads 2 of 4

Year: Feb-15 
Slogan: "Safety First" means a tripod for all exposures over 1-25 of a second.

Subject(s): product - misc.

Product: Kodak Metal tripod

Description / Notes: Price from $\$ 1.60$ to $\$ 3.25$. Leather carrying case: $\$ 1.50$

Binder: 1913-1917 Amateur Ads 2 of 4

Year: Dec-14

Slogan: "Safety First" means a tripod / The Kodiopticon

Subject(s): product - misc.

Product: Kodak Metal tripod / Kodiopticon

Color or Black and White:

Binder: 1913-1917 Amateur Ads 2 of 4

Year: Jan-15

Slogan: All In-Doors invites your Kodak when it's fitted with The

Kodak Portrait Attachment

Subject(s): product - camera

Product: Kodak portrait attachment

Description / Notes: Price: $\$ 0.50$

Binder: 1913-1917 Amateur Ads 2 of 4

Year: Jun-15

Slogan: Nature's best interpreter of nature at her best--the Kodak

and the Kodak Portrait Attachment

Subject(s): product - camera

Product: Kodak Portrait Attachment

Description / Notes: price $\$ 0.50$

Binder: 1913-1917 Amateur Ads 2 of 4

Year: Oct-14

Slogan: Add to the attractiveness of your vacation Kodak Album with prints on Kodak Velvet Green Paper

Subject(s): product - film \& paper

Product: Kodak Velvet Green Paper

Color or Black and White:

Binder: 1913-1917 Amateur Ads 2 of 4
Year: May-15

Slogan: Print your landscapes and seascapes on Kodak Velvet

Green paper

Subject(s): product - film \& paper

Product: Kodak Velvet Green Paper

Color or Black and White:

Binder: 1913-1917 Amateur Ads 2 of 4

Year: Jun-15

Slogan: The Tint's in the Print when you use Kodak Velvet Green

Paper

Subject(s): product - film \& paper

Product: Kodak Velvet Green Paper

Color or Black and White:

Binder: 1913-1917 Amateur Ads 2 of 4

Year: Feb-15

Slogan: With the introduction of Nos. 1 and 1a Autographic Kodak Juniors, the line of Autographic Kodaks in the popular sizes is now complete.

Subject(s): product - camera

Product: No. 1 Autographic Kodak Junior, No. 1a Autographic

Kodak Junior

Description / Notes: prices from $\$ 9.00$ to $\$ 27.50$

Binder: 1913-1917 Amateur Ads 2 of 4

Year: Jun-15

Slogan: 3,000.00 in cash prizes for pictures illustrating Kodak

advertising slogans

Subject(s): product - misc

Product: Photo contest

Description / Notes: Prize for the best photo illustrating one of five slogans: $\$ 300.00$. Five slogans: "Take a Kodak with you. All Out-Doors invites your Kodak. There are no game laws for those who hunt with a Kodak. Let the children Kodak. Write it on the film--at the time." Also, $\$ 500.00$ prize for the best new slogan and photo accompanying it.

Binder: 1913-1917 Amateur Ads 2 of 4 
Year: Mar-15

Slogan: Pocketfoto

Subject(s): product - misc.

Product: Pocketfoto

Description / Notes: Price: from $\$ 1.25$ to $\$ 1.75$

Binder: 1913-1917 Amateur Ads 2 of 4

Year: Feb-15

Slogan: The R.O.C. Enlarging Back

Subject(s): product - camera

Product: R.O.C. Enlarging Back

Description / Notes: Price: $\$ 8.00$

Binder: 1913-1917 Amateur Ads 2 of 4

Year: Dec-14

Slogan: Twenty Minutes in a Rounds Print washer

Subject(s): product - darkroom

Product: Rounds Print Washer

Description / Notes: Price for 100 capacity: $\$ 10.00$. For 200

capacity: $\$ 20.00$

Binder: 1913-1917 Amateur Ads 2 of 4

Year: Apr-15

Slogan: For every occasion where extreme speed is essential use the Seed Graflex Plate

Subject(s): product - camera

Product: Seed Dry Plates

Color or Black and White:

Binder: 1913-1917 Amateur Ads 2 of 4

Year: Nov-14

Slogan: Seed Graflex Plate

Subject(s): product - film \& paper

Product: Seed Dry Plates

Color or Black and White:

Binder: 1913-1917 Amateur Ads 2 of 4

Year: Apr-15

Slogan: The Souvenir Photo Case
Subject(s): product - misc.

Product: Souvenir Photo Case

Description / Notes: Price: from $\$ 0.35$ to $\$ 0.50$

Binder: 1913-1917 Amateur Ads 2 of 4

Year: Feb-15

Slogan: Strong--Practical--Convenient

Subject(s): product - misc.

Product: The Eastman Studio Reflector

Description / Notes: Price: $\$ 12.00$

Binder: 1913-1917 Amateur Ads 2 of 4

Year: Jan-15

Slogan: The picture worth taking is worth keeping

Subject(s): product - misc.

Product: The Kodak Album

Description / Notes: article.

Binder: 1913-1917 Amateur Ads 2 of 4

Year: Dec-14

Slogan: Almost another camera for fifty cents

Subject(s): product - camera

Product: The Kodak Portrait Attachment

Description / Notes: price: $\$ 0.50$

Binder: 1913-1917 Amateur Ads 2 of 4

Year: Feb-15

Slogan: Does Double Duty. Thermometer Stirring Rod

Subject(s): product - darkroom

Product: Thermometer Stirring Rod

Description / Notes: Price: $\$ 0.60$

Binder: 1913-1917 Amateur Ads 2 of 4

Year: Jun-15

Slogan: With the Thermometer Stirring Rod

Subject(s): product - darkroom

Product: Thermometer Stirring Rod

Description / Notes: price: $\$ 0.60$ 
Binder: 1913-1917 Amateur Ads 2 of 4

Year: Oct-14

Slogan: Tozol - The Complete Developing Agent for photographic Papers

Subject(s): product - Chemicals

Product: Tozol Developing Agent

Description / Notes: 1 oz bottle price: $\$ 0.40 .1 / 4$ pound: $\$ 1.40$. $1 / 2$ Pound: $\$ 2.60 .1$ pound: $\$ 5.00$

Binder: 1913-1917 Amateur Ads 2 of 4

Year: Nov-14

Slogan: The Logical Decision Velox

Subject(s): product - film \& paper

Product: Velox

Color or Black and White:

Binder: 1913-1917 Amateur Ads 2 of 4

Year: Jan-15

Slogan: What's in the negative comes out in the print when you use Velox

Subject(s): product - film \& paper

Product: velox

Color or Black and White:

Binder: 1913-1917 Amateur Ads 2 of 4

Year: Jun-15

Slogan: Velox a photographic paper that fits

Subject(s): product - film \& paper

Product: Velox

Color or Black and White:

Binder: 1913-1917 Amateur Ads 2 of 4

Year: Oct-14

Slogan: For the best possible print: Velox

Subject(s): product - film \& paper

Product: Velox paper

Color or Black and White:

Binder: 1913-1917 Amateur Ads 2 of 4
Year: Nov-14

Slogan: Made with you in mind. Velox.

Subject(s): product - film \& paper

Product: Velox paper

Color or Black and White:

Binder: 1913-1917 Amateur Ads 2 of 4

Year: Jan-15

Slogan: The paper that meets the amateur half-way, Velox

Subject(s): product - film \& paper

Product: Velox paper

Color or Black and White:

Binder: 1913-1917 Amateur Ads 2 of 4

Year: Mar-15

Slogan: The last word in photographic printing is Velox

Subject(s): product - film \& paper

Product: Velox paper

Color or Black and White:

Binder: 1913-1917 Amateur Ads 2 of 4

Year: Apr-15

Slogan: There's success in every sheet of Velox the Amateur's

Own Paper

Subject(s): product - film \& paper

Product: Velox paper

Color or Black and White:

Binder: 1913-1917 Amateur Ads 2 of 4

Year: Jun-15

Slogan: As far as photographic printing goes, results and Velox

mean one and the same thing

Subject(s): product - film \& paper

Product: Velox paper

Color or Black and White:

Binder: 1913-1917 Amateur Ads 2 of 4

Year: Oct-14

Slogan: Color your choice prints with Velox Transparent Water 
Color Stamps

Subject(s): product - misc.

Product: Velox Transparent Water Color Stamps

Description / Notes: Book of stamps price: $\$ 0.25$. Complete set price: $\$ 0.75$

Binder: 1913-1917 Amateur Ads 2 of 4

Year: Nov-14

Slogan: Why not green fields and a blue sky rather than a

landscape in black and white

Subject(s): product - misc.

Product: Velox Transparent Water Color Stamps

Color or Black and White:

Binder: 1913-1917 Amateur Ads 2 of 4

Year: May-15

Slogan: A touch of nature with every stroke of the brush if you color your landscapes with Velox transparent water color stamps Subject(s): product - misc.

Product: Velox Transparent Water Color Stamps

Description / Notes: Price per book of stamps: $\$ 0.25$. Complete outfit: $\$ 0.75$

Binder: 1913-1917 Amateur Ads 2 of 4

Year: Feb-15

Slogan: "That Wonderful Little Camera"

Subject(s): product - camera

Product: Vest Pocket Kodak

Description / Notes: Article.

Binder: 1913-1917 Amateur Ads 2 of 4

Year: Apr-15

Slogan: "Oh if I only had a Kodak"

Subject(s): product - camera

Product: Vest Pocket Kodak

Description / Notes: Article.

Binder: 1913-1917 Amateur Ads 2 of 4

Year: May-15
Slogan: The large print The easy way with the Vest Pocket Kodak or Brownie Enlarging Camera

Subject(s): product - camera

Product: Vest Pocket Kodak Enlarging Camera, Brownie Enlarging Camera

Description / Notes: Price: from $\$ 2.25$ to $\$ 3.60$

Binder: 1913-1917 Amateur Ads 3 of 4

Year: Oct-15

Slogan: To know "How to Make Good Pictures" read it.

Subject(s): product - misc.

Product: "How to Make Good Pictures" booklet

Description / Notes: article.

Binder: 1913-1917 Amateur Ads 3 of 4

Year: Apr-16

Slogan: Send for this Book.

Subject(s): product - misc.

Product: 1906 Graflex catalog

Color or Black and White:

Binder: 1913-1917 Amateur Ads 3 of 4

Year: Apr-16

Slogan: The New 3a Autographic Kodak Special with Range Finder

Subject(s): product - camera

Product: 3a Autographic Kodak

Description / Notes: Price: $\$ 66.00$ and $\$ 77.00$

Binder: 1913-1917 Amateur Ads 3 of 4

Year: Mar-16

Slogan: The New 3a Kodak

Subject(s): product - camera

Product: 3a Kodak

Description / Notes: Price: $\$ 27.50$

Binder: 1913-1917 Amateur Ads 3 of 4

Year: Oct-15

Slogan: Large Prints on Artura Carbon Black

Subject(s): product - film \& paper 
Product: Artura Carbon Black

Color or Black and White:

Binder: 1913-1917 Amateur Ads 3 of 4

Year: Jan-16

Slogan: Duplicate the small print in larger size on Artura Carbon

Black

Subject(s): product - film \& paper

Product: Artura Carbon Black

Color or Black and White:

Binder: 1913-1917 Amateur Ads 3 of 4

Year: Apr-16

Slogan: Artura Carbon Black

Subject(s): product - film \& paper

Product: Artura Carbon Black

Color or Black and White:

Binder: 1913-1917 Amateur Ads 3 of 4

Year: Aug-15

Slogan: Artura Quality all the way through

Subject(s): product - film \& paper

Product: Artura Paper

Color or Black and White:

Binder: 1913-1917 Amateur Ads 3 of 4

Year: Oct-15

Slogan: A New Grade of Artura Iris

Subject(s): product - film \& paper

Product: Artura Paper

Color or Black and White:

Binder: 1913-1917 Amateur Ads 3 of 4

Year: Dec-15

Slogan: The success based on superiority. Artura

Subject(s): product - film \& paper

Product: Artura Paper

Color or Black and White:
Binder: 1913-1917 Amateur Ads 3 of 4

Year: Feb-16

Slogan: The Man who uses Artura

Subject(s): product - film \& paper

Product: Artura Paper

Color or Black and White:

Binder: 1913-1917 Amateur Ads 3 of 4

Year: Mar-16

Slogan: You have a positive assurance if the print is on Artura.

Subject(s): product - film \& paper

Product: Artura Paper

Color or Black and White:

Binder: 1913-1917 Amateur Ads 3 of 4

Year: May-16

Slogan: Artura

Subject(s): product - film \& paper

Product: Artura Paper

Color or Black and White:

Binder: 1913-1917 Amateur Ads 3 of 4

Year: Oct-15

Slogan: Your Kodak becomes Autographic

Subject(s): product - camera

Product: Autographic Backs for Kodaks

Description / Notes: price: from $\$ 2.50$ to $\$ 4.50$

Binder: 1913-1917 Amateur Ads 3 of 4

Year: May-16

Slogan: Another Autographic Brownie

Subject(s): product - film \& paper

Product: Autographic Brownie

Description / Notes: Article.

Binder: 1913-1917 Amateur Ads 3 of 4

Year: Apr-16

Slogan: An autobiography with an Autographic Kodak

Subject(s): product - camera 
Product: Autographic Kodak

Description / Notes: Article.

Binder: 1913-1917 Amateur Ads 3 of 4

Year: Aug-15

Slogan: The construction of the Brownie Enlarging Camera

Subject(s): product - camera

Product: Brownie Enlarging Camera

Description / Notes: Price: from $\$ 1.75$ to $\$ 4.00$

Binder: 1913-1917 Amateur Ads 3 of 4

Year: Nov-15

Slogan: To obtain large prints with the Brownie Enlarging Camera

Subject(s): product - camera

Product: Brownie Enlarging Camera

Description / Notes: price: from $\$ 1.75$ to $\$ 4.00$

Binder: 1913-1917 Amateur Ads 3 of 4

Year: Feb-16

Slogan: The Brownie Enlarging Camera

Subject(s): product - camera

Product: Brownie Enlarging Camera

Description / Notes: Price from $\$ 1.75$ to $\$ 4.00$

Binder: 1913-1917 Amateur Ads 3 of 4

Year: Sep-15

Slogan: Brownie Enlarging Camera Illuminator

Subject(s): product - camera

Product: Brownie Enlarging Camera Illuminator

Description / Notes: Price: $\$ 3.00$

Binder: 1913-1917 Amateur Ads 3 of 4

Year: Jun-16

Slogan: The Brownie Safelight

Subject(s): product - darkroom

Product: Brownie Safelight

Description / Notes: Price: $\$ 1.00$

Binder: 1913-1917 Amateur Ads 3 of 4
Year: Apr-16

Slogan: Developing Conveniences. Core plate developing racks

Subject(s): product - film \& paper

Product: Core plate developing racks, Eastman film developing box No. 2.

Description / Notes: Price for racks: from $\$ 0.50$ to $\$ 1.60$. Price for developing box: $\$ 3.00$

Binder: 1913-1917 Amateur Ads 3 of 4

Year: Oct-15

Slogan: A good enlarging outfit will yield bigger returns on your investment than any one piece of apparatus you can install in your studio.

Subject(s): product - camera

Product: Eastman Enlarging Outfit

Description / Notes: Price complete outfit: $\$ 85.00$

Binder: 1913-1917 Amateur Ads 3 of 4

Year: Oct-15

Slogan: The right album for your negatives

Subject(s): product - film \& paper

Product: Eastman Film Negative Album

Description / Notes: price: from $\$ 0.75$ to $\$ 1.50$

Binder: 1913-1917 Amateur Ads 3 of 4

Year: Nov-15

Slogan: Used in connection with the Kodak Flash Sheet Holder.

Eastman Flash Sheets

Subject(s): product - misc.

Product: Eastman Flash Sheets

Color or Black and White:

Binder: 1913-1917 Amateur Ads 3 of 4

Year: Feb-16

Slogan: With Eastman Flash Sheets and the Kodak Flash Sheet Holder

Subject(s): product - misc

Product: Eastman Flash Sheets

Description / Notes: Price for Flash sheet holder: \$1.00 
Binder: 1913-1917 Amateur Ads 3 of 4

Year: Aug-15

Slogan: The Mark of Distinction

Subject(s): product - Chemicals

Product: Eastman Kodak Company Tested Chemicals

Color or Black and White:

Binder: 1913-1917 Amateur Ads 3 of 4

Year: Sep-15

Slogan: The Seal that inspires confidence

Subject(s): product - Chemicals

Product: Eastman Kodak Company Tested Chemicals

Color or Black and White:

Binder: 1913-1917 Amateur Ads 3 of 4

Year: Oct-15

Slogan: This seal, on every bottle or tin, insures its quality

Subject(s): product - Chemicals

Product: Eastman Kodak Company Tested Chemicals

Color or Black and White:

Binder: 1913-1917 Amateur Ads 3 of 4

Year: Dec-15

Slogan: When you see this seal on the container:

Subject(s): product - Chemicals

Product: Eastman Kodak Company Tested Chemicals

Color or Black and White:

Binder: 1913-1917 Amateur Ads 3 of 4

Year: Dec-15

Slogan: The chemical in its most convenient form: Eastman Permanent Crystal Pyro

Subject(s): product - Chemicals

Product: Eastman Kodak Company Tested Chemicals

Color or Black and White:

Binder: 1913-1917 Amateur Ads 3 of 4

Year: Mar-16
Slogan: E.K. Tested Chemicals

Subject(s): product - Chemicals

Product: Eastman Kodak Company Tested Chemicals

Color or Black and White:

Binder: 1913-1917 Amateur Ads 3 of 4

Year: Mar-16

Slogan: Specify Eastman Permanent Crystal Pyro in your next order

Subject(s): product - Chemicals

Product: Eastman Kodak Company Tested Chemicals

Color or Black and White:

Binder: 1913-1917 Amateur Ads 3 of 4

Year: Apr-16

Slogan: The seal with the certainty back of it

Subject(s): product - Chemicals

Product: Eastman Kodak Company Tested Chemicals

Color or Black and White:

Binder: 1913-1917 Amateur Ads 3 of 4

Year: May-16

Slogan: We are both Certain

Subject(s): product - Chemicals

Product: Eastman Kodak Company Tested Chemicals

Color or Black and White:

Binder: 1913-1917 Amateur Ads 3 of 4

Year: Jan-16

Slogan: Begin the New Year with a New Studio Register

Subject(s): product - misc.

Product: Eastman Loose Leaf Studio Register / Eastman Card Index Studio Register

Description / Notes: Price for Loose leaf register: \$2.50. Price for Index card register: $\$ 5.00$

Binder: 1913-1917 Amateur Ads 3 of 4

Year: Aug-15

Slogan: The photographer who knows and appreciates quality, 
sticks once he has used Eastman Portrait Film

Subject(s): product - film \& paper

Product: Eastman Portrait Film

Color or Black and White:

Binder: 1913-1917 Amateur Ads 3 of 4

Year: Oct-15

Slogan: None

Subject(s): product - film \& paper

Product: Eastman Portrait Film

Description / Notes: A Letter from H. Walter Barnett to Kodak,

endorsing Eastman Portrait Film. "is yielding splendid results"

Binder: 1913-1917 Amateur Ads 3 of 4

Year: Nov-15

Slogan: The real advantage of Eastman Portrait Film

Subject(s): product - film \& paper

Product: Eastman Portrait Film

Color or Black and White:

Binder: 1913-1917 Amateur Ads 3 of 4

Year: Nov-15

Slogan: Doubled!

Subject(s): product - film \& paper

Product: Eastman Portrait Film

Color or Black and White:

Binder: 1913-1917 Amateur Ads 3 of 4

Year: Feb-16

Slogan: $160 \%$ Increase.

Subject(s): product - film \& paper

Product: Eastman Portrait Film

Color or Black and White:

Binder: 1913-1917 Amateur Ads 3 of 4

Year: Jan-16

Slogan: Developing and Printing Outfits

Subject(s): product - darkroom

Product: Eastman printing outfits
Description / Notes: Price for Eastman 3a outfit: $\$ 2.15$. Price for Eastman A B C outfit: $\$ 1.50$. Brownie Developing and Printing Outfit: $\$ 0.90$

Binder: 1913-1917 Amateur Ads 3 of 4

Year: Mar-16

Slogan: Eastman Studio Reflector

Subject(s): product - misc.

Product: Eastman Studio Reflector

Description / Notes: Price: $\$ 12.00$

Binder: 1913-1917 Amateur Ads 3 of 4

Year: Aug-15

Slogan: To keep the quality right, keep the time and temperature right.

Subject(s): product - darkroom

Product: Eastman Thermometer Stirring Rod

Description / Notes: Price: $\$ 0.60$

Binder: 1913-1917 Amateur Ads 3 of 4

Year: Jun-16

Slogan: Even Kodak Tested Chemicals can't do their work properly

if the solutions are too warm. Here are two reliable means of

checking up the temperature.

Subject(s): product - darkroom

Product: Eastman Thermometer, Thermometer Stirring Rod

Description / Notes: Thermometer price: $\$ 0.65$. Thermometer

Stirring Rod price: $\$ 0.75$.

Binder: 1913-1917 Amateur Ads 3 of 4

Year: Mar-16

Slogan: There is just one temperature at which a solution does its best work.

Subject(s): product - misc

Product: Eastman Thermometer, Thermometer Stirring Rod

Description / Notes: Price for thermometer: $\$ 0.50$. Price for

Thermometer stirring Rod: $\$ 0.60$

Binder: 1913-1917 Amateur Ads 3 of 4 
Year: Apr-16

Slogan: The readings are plain as day--in the darkroom with the Eastman Visible Graduate

Subject(s): product - darkroom

Product: Eastman visible graduate

Description / Notes: Price: from $\$ 0.20$ to $\$ 0.80$

Binder: 1913-1917 Amateur Ads 3 of 4

Year: May-16

Slogan: The black opaque gradations on the Eastman Visible graduate

Subject(s): product - darkroom

Product: Eastman visible graduate

Description / Notes: Price: from $\$ 0.20$ to $\$ 0.80$

Binder: 1913-1917 Amateur Ads 3 of 4

Year: Aug-15

Slogan: The combination of the Kodak Amateur Printer and the Kodak Film Tank has made every amateur his own finishing department

Subject(s): product - darkroom

Product: Kodak Amateur Printer / Kodak Film Tank

Description / Notes: Price for amateur printer: $\$ 5.00$

Binder: 1913-1917 Amateur Ads 3 of 4

Year: Dec-15

Slogan: "Every Amateur His Own Finishing Department"

Subject(s): product - darkroom

Product: Kodak Amateur Printer / Kodak Film Tank

Description / Notes: Price for Film Tank: $\$ 2.50$ to $\$ 7.50$. Price for printer: $\$ 5.00$

Binder: 1913-1917 Amateur Ads 3 of 4

Year: Jan-16

Slogan: Another Kodak Convenience

Subject(s): product - darkroom

Product: Kodak Amateur Printer / Kodak Film Tank

Description / Notes: article.
Binder: 1913-1917 Amateur Ads 3 of 4

Year: Apr-16

Slogan: The combination of the Kodak Amateur Printer and the

Kodak Film Tank makes every amateur his own finishing

department

Subject(s): product - darkroom

Product: Kodak Amateur Printer / Kodak Film Tank

Color or Black and White:

Binder: 1913-1917 Amateur Ads 3 of 4

Year: Sep-15

Slogan: The Kodak Autotime Scale

Subject(s): product - camera

Product: Kodak Autotime Scale

Color or Black and White:

Binder: 1913-1917 Amateur Ads 3 of 4

Year: Jan-16

Slogan: Two Darkroom lamps that give you all the light you want with all the safety you need.

Subject(s): product - darkroom

Product: Kodak Dark Room Lamp / Kodak Candle Lamp

Description / Notes: Price for darkroom lamp: \$1.00. Price for Candle lamp: \$0.25

Binder: 1913-1917 Amateur Ads 3 of 4

Year: Oct-15

Slogan: Kodak Dry Mounting Tissue

Subject(s): product - film \& paper

Product: Kodak Dry Mounting Tissue

Description / Notes: price: from $\$ 0.20$ to $\$ 1.50$

Binder: 1913-1917 Amateur Ads 3 of 4

Year: Aug-15

Slogan: The Kodak Film Tank has made the darkroom a relic and better negatives the rule.

Subject(s): product - darkroom

Product: Kodak film tank

Description / Notes: Price: from $\$ 2.50$ to $\$ 7.50$ 
Binder: 1913-1917 Amateur Ads 3 of 4

Year: Dec-15

Slogan: The Kodak Film Tank

Subject(s): product - darkroom

Product: Kodak Film Tank

Description / Notes: Article.

Binder: 1913-1917 Amateur Ads 3 of 4

Year: Mar-16

Slogan: The novice who used a Kodak Film Tank

Subject(s): product - darkroom

Product: Kodak film tank

Color or Black and White:

Binder: 1913-1917 Amateur Ads 3 of 4

Year: Jun-16

Slogan: Take a Kodak Film Tank With you

Subject(s): product - darkroom

Product: Kodak Film Tank

Description / Notes: Price: from \$2.75 up.

Binder: 1913-1917 Amateur Ads 3 of 4

Year: Jan-16

Slogan: Kodak Anastigmat f.7.7

Subject(s): product - camera

Product: Kodak lenses.

Color or Black and White:

Binder: 1913-1917 Amateur Ads 3 of 4

Year: Dec-15

Slogan: Kodak Magnesium Ribbon Holder

Subject(s): product - darkroom

Product: Kodak Magnesium Ribbon Holder

Description / Notes: price for ribbon holder: $\$ 0.20$ alcohol lamp: $\$ 0.20$

Binder: 1913-1917 Amateur Ads 3 of 4

Year: Oct-15
Slogan: Where the hand fails use a Kodak Metal Tripod

Subject(s): product - misc.

Product: Kodak Metal tripod

Description / Notes: price: from $\$ 1.60$ to $\$ 3.25$. Leather carrying case: $\$ 1.50$

Binder: 1913-1917 Amateur Ads 3 of 4

Year: Dec-15

Slogan: The New Kodak Metal Tripod

Subject(s): product - misc.

Product: Kodak Metal tripod

Description / Notes: price: $\$ 5.00$

Binder: 1913-1917 Amateur Ads 3 of 4

Year: Jan-16

Slogan: With the Kodak Portrait Attachment

Subject(s): product - camera

Product: Kodak Portrait Attachment

Description / Notes: price: $\$ 0.50$

Binder: 1913-1917 Amateur Ads 3 of 4

Year: Mar-16

Slogan: By increasing the Kodak's field of usefulness, The Kodak

Portrait Attachment

Subject(s): product - camera

Product: Kodak Portrait Attachment

Description / Notes: Price: $\$ 0.50$

Binder: 1913-1917 Amateur Ads 3 of 4

Year: Apr-16

Slogan: Close to Nature with a Kodak and Kodak Portrait

Attachment

Subject(s): product - camera

Product: Kodak Portrait Attachment

Description / Notes: price: $\$ 0.50$

Binder: 1913-1917 Amateur Ads 3 of 4

Year: Mar-16

Slogan: Kodak Safelight Lamp 
Subject(s): product - darkroom

Product: Kodak Safelight Lamp

Description / Notes: price $\$ 3.00$

Binder: 1913-1917 Amateur Ads 3 of 4

Year: May-16

Slogan: The photographer uses a lamp similar in construction to the Kodak safelight lamp

Subject(s): product - darkroom

Product: Kodak Safelight Lamp

Description / Notes: Price: $\$ 3.00$

Binder: 1913-1917 Amateur Ads 3 of 4

Year: Sep-15

Slogan: The Upper Half of the Kodak Sky Filter

Subject(s): product - camera

Product: Kodak Sky Filter

Description / Notes: Price from $\$ 0.50$ to $\$ 1.00$

Binder: 1913-1917 Amateur Ads 3 of 4

Year: Feb-16

Slogan: The Significance of Kodak

Subject(s): product - film \& paper

Product: Kodak System

Description / Notes: Article.

Binder: 1913-1917 Amateur Ads 3 of 4

Year: Feb-16

Slogan: Two Kodak Conveniences

Subject(s): product - misc.

Product: Kodak Trimming Board / Kodak Magnesium Ribbon Holder

Description / Notes: Price for trimming board: $\$ 0.40$ and $\$ 0.60$

Price for Ribbon Holder: $\$ 0.20$.

Binder: 1913-1917 Amateur Ads 3 of 4

Year: Oct-15

Slogan: The rule, steel blade, and exact construction of the Kodak Trimming Board
Subject(s): product - misc.

Product: Kodak trimming boards

Description / Notes: price: $5 \times 5$ inches: $\$ 0.40 .7 \times 7$ inches: $\$ 0.60$

Binder: 1913-1917 Amateur Ads 3 of 4

Year: Apr-16

Slogan: With the first hint of spring, Kodak Velvet Green

Subject(s): product - film \& paper

Product: Kodak Velvet Green Paper

Color or Black and White:

Binder: 1913-1917 Amateur Ads 3 of 4

Year: May-16

Slogan: The tint's in the print on Kodak Velvet Green

Subject(s): product - film \& paper

Product: Kodak Velvet Green Paper

Color or Black and White:

Binder: 1913-1917 Amateur Ads 3 of 4

Year: Apr-16

Slogan: Kodak Dominates

Subject(s): product - misc.

Product: Kodak Workmanship

Color or Black and White:

Binder: 1913-1917 Amateur Ads 3 of 4

Year: Aug-15

Slogan: Improved Majestic Print Dryers

Subject(s): product - misc.

Product: Majestic Print Dryer

Description / Notes: Price for No 1: $\$ 25.00$. Price for No. 5 : $\$ 50.00$

Binder: 1913-1917 Amateur Ads 3 of 4

Year: Nov-15

Slogan: No. 1 Autographic Kodak, Special

Subject(s): product - camera

Product: No. 1 Autographic Kodak, Special

Description / Notes: price: $\$ 45.00$ 
Binder: 1913-1917 Amateur Ads 3 of 4

Year: May-16

Slogan: The No. 1 Eastman Printer

Subject(s): product - darkroom

Product: No. 1 Eastman printer

Description / Notes: price: $\$ 10.00$

Binder: 1913-1917 Amateur Ads 3 of 4

Year: Jan-16

Slogan: Nos. 2 and 2a Folding Autographic Brownies

Subject(s): product - camera

Product: No. 2 and 2a Folding Autographic Brownies

Description / Notes: Price: $\$ 6.00$ to $\$ 10.00$

Binder: 1913-1917 Amateur Ads 3 of 4

Year: Oct-15

Slogan: The New No. 2 Folding Autographic Brownie

Subject(s): product - camera

Product: No. 2 Folding Autographic Brownie

Description / Notes: Price: $\$ 6.00$

Binder: 1913-1917 Amateur Ads 3 of 4

Year: May-16

Slogan: The No. 2c Folding Autographic Brownie

Subject(s): product - camera

Product: No. 2c Folding Autographic Brownie

Description / Notes: price: $\$ 9.00$ and $\$ 11.00$

Binder: 1913-1917 Amateur Ads 3 of 4

Year: Sep-15

Slogan: The Panoram Kodaks

Subject(s): product - camera

Product: Panoram Kodak No.1 and No. 4

Description / Notes: Price for No. 1 Panoram Kodak: $\$ 10.00$.

Price for No. 4 Panoram Kodak: $\$ 20.00$

Binder: 1913-1917 Amateur Ads 3 of 4

Year: Mar-16
Slogan: The Panoram Kodaks

Subject(s): product - camera

Product: Panoram Kodak No.1 and No. 4

Description / Notes: Article.

Binder: 1913-1917 Amateur Ads 3 of 4

Year: Sep-15

Slogan: Three Thousand Dollars For Pictures

Subject(s): product - misc.

Product: Photo contest

Description / Notes: Article.

Binder: 1913-1917 Amateur Ads 3 of 4

Year: Oct-15

Slogan: Here is opportunity

Subject(s): product - misc

Product: Photo contest

Description / Notes: Prize for the best photo illustrating one of five slogans: $\$ 300.00$. Five slogans: "Take a Kodak with you. All Out-Doors invites your Kodak. There are no game laws for those who hunt with a Kodak. Let the children Kodak. Write it on the film--at the time." Also, $\$ 500.00$ prize for the best new slogan and photo accompanying it. Contest closes November 1st

Binder: 1913-1917 Amateur Ads 3 of 4

Year: Oct-15

Slogan: The only Grand Prize

Subject(s): product - misc.

Product: Photo contest

Description / Notes: The grand prize at the Panama-Pacific Exposition, awarded to Eastman Kodak Company.

Binder: 1913-1917 Amateur Ads 3 of 4

Year: May-16

Slogan: The 1916 Kodak Advertising Competition

Subject(s): product - misc.

Product: Photo contest

Description / Notes: "3,000.00 CASH for pictures best

illustrating Kodak advertisements." Contest closes November 1st 
Binder: 1913-1917 Amateur Ads 3 of 4

Year: Apr-16

Slogan: Plastic Plates

Subject(s): product - film \& paper

Product: Plastic Plates

Description / Notes: The re-coating of old plates for all plates except: Seed, Standard, Stanley, Weatten \& Wainwright, and

Eastman Plates.

Binder: 1913-1917 Amateur Ads 3 of 4

Year: Nov-15

Slogan: A substantial trimmer for small and medium sized prints at a very reasonable price.

Subject(s): product - misc.

Product: R.O.C. Trimmer

Description / Notes: price: $\$ 1.50$ to $\$ 2.00$

Binder: 1913-1917 Amateur Ads 3 of 4

Year: Nov-15

Slogan: Increase your efficiency--deliver your work promptly.

Subject(s): product - misc.

Product: Rounds print washer, Improved majestic print dryer

Description / Notes: Price for washer: $\$ 10.00, \$ 30.00$. Print for dryer: $\$ 25.00$ and $\$ 50.00$

Binder: 1913-1917 Amateur Ads 3 of 4

Year: Aug-15

Slogan: Seed Plates

Subject(s): product - film \& paper

Product: Seed Dry Plates

Color or Black and White:

Binder: 1913-1917 Amateur Ads 3 of 4

Year: Jan-16

Slogan: Super-speed best describes the extreme sensitiveness of the Seed Graflex Plate

Subject(s): product - film \& paper

Product: Seed Dry Plates

\section{Color or Black and White}

Binder: 1913-1917 Amateur Ads 3 of 4

Year: Feb-16

Slogan: Seed Quality

Subject(s): product - film \& paper

Product: Seed Dry Plates

Color or Black and White

Binder: 1913-1917 Amateur Ads 3 of 4

Year: Dec-15

Slogan: For the commercial photographer: Two New Standard

Plates

Subject(s): product - film \& paper

Product: Standard Dry Plate

Color or Black and White:

Binder: 1913-1917 Amateur Ads 3 of 4

Year: Aug-15

Slogan: The Eastman Film Negative Album

Subject(s): product - misc.

Product: The Eastman Film Negative Album

Description / Notes: Price: from $\$ 0.75$ to $\$ 1.50$

Binder: 1913-1917 Amateur Ads 3 of 4

Year: Oct-15

Slogan: The Right album for your prints

Subject(s): product - film \& paper

Product: The Kodak Album

Description / Notes: Price: from $\$ 2.25$ to $\$ 3.50$

Binder: 1913-1917 Amateur Ads 3 of 4

Year: Jul-15

Slogan: You'll want an album

Subject(s): product - misc.

Product: The Kodak Album

Description / Notes: article.

Binder: 1913-1917 Amateur Ads 3 of 4 
Year: Dec-15

Slogan: Keep a Photographic Diary

Subject(s): product - misc.

Product: The Kodak Album

Description / Notes: Price: from $\$ 2.25$ to $\$ 3.50$

Binder: 1913-1917 Amateur Ads 3 of 4

Year: Nov-15

Slogan: Holiday Hints

Subject(s): product - misc.

Product: The Kodak Album / Year Book calendar

Color or Black and White:

Binder: 1913-1917 Amateur Ads 3 of 4

Year: Dec-15

Slogan: Day in and day out, the Kodak Film Tank

Subject(s): product - darkroom

Product: The Kodak Film Tank

Color or Black and White:

Binder: 1913-1917 Amateur Ads 3 of 4

Year: Dec-15

Slogan: A few gift suggestions for your friend, the amateur

Subject(s): product - misc.

Product: The Kodak System

Color or Black and White:

Binder: 1913-1917 Amateur Ads 3 of 4

Year: Mar-16

Slogan: Two first aids to the time exposure:

Subject(s): product - misc.

Product: The Universal clamp, The new Kodak Metal Tripod

Description / Notes: Price for clamp: $\$ 0.75$. Price for Tripod $\$ 5.00$

Binder: 1913-1917 Amateur Ads 3 of 4

Year: Nov-15

Slogan: The Year Book Calendar

Subject(s): product - misc.
Product: The Year Book Calendar

Description / Notes: price: $\$ 0.50$

Binder: 1913-1917 Amateur Ads 3 of 4

Year: Dec-15

Slogan: Make gifts that bear the personal touch.

Subject(s): product - misc.

Product: The Year Book Calendar

Description / Notes: price: $\$ 0.50$

Binder: 1913-1917 Amateur Ads 3 of 4

Year: Oct-15

Slogan: Velox offers all that your negatives demand

Subject(s): product - film \& paper

Product: Velox

Color or Black and White:

Binder: 1913-1917 Amateur Ads 3 of 4

Year: Aug-15

Slogan: Results follow as surely as the night the day if you make your prints on Velox

Subject(s): product - film \& paper

Product: Velox paper

Color or Black and White

Binder: 1913-1917 Amateur Ads 3 of 4

Year: Sep-15

Slogan: Contrast Velox

Subject(s): product - film \& paper

Product: Velox paper

Color or Black and White:

Binder: 1913-1917 Amateur Ads 3 of 4

Year: Nov-15

Slogan: Velox the photographic paper that fits.

Subject(s): product - film \& paper

Product: Velox paper

Color or Black and White: 
Binder: 1913-1917 Amateur Ads 3 of 4

Year: Dec-15

Slogan: Say "Velox"

Subject(s): product - film \& paper

Product: Velox paper

Color or Black and White:

Binder: 1913-1917 Amateur Ads 3 of 4

Year: Dec-15

Slogan: Simple in manipulation, Dependable in results, Velox.

Subject(s): product - film \& paper

Product: Velox paper

Color or Black and White:

Binder: 1913-1917 Amateur Ads 3 of 4

Year: Feb-16

Slogan: Just one good print after another with Velox

Subject(s): product - film \& paper

Product: Velox paper

Color or Black and White:

Binder: 1913-1917 Amateur Ads 3 of 4

Year: Mar-16

Slogan: Velox is the best print you can get

Subject(s): product - film \& paper

Product: Velox paper

Color or Black and White:

Binder: 1913-1917 Amateur Ads 3 of 4

Year: Apr-16

Slogan: There are as many reasons for Velox

Subject(s): product - film \& paper

Product: Velox paper

Color or Black and White:

Binder: 1913-1917 Amateur Ads 3 of 4

Year: May-16

Slogan: Made to your order: Velox

Subject(s): product - film \& paper
Product: Velox paper

Color or Black and White:

Binder: 1913-1917 Amateur Ads 3 of 4

Year: May-16

Slogan: The Versatility of Velox

Subject(s): product - film \& paper

Product: Velox Paper

Color or Black and White:

Binder: 1913-1917 Amateur Ads 3 of 4

Year: Aug-15

Slogan: Color your vacation prints

Subject(s): product - misc.

Product: Velox Transparent Water Color Stamps

Description / Notes: Article.

Binder: 1913-1917 Amateur Ads 3 of 4

Year: Nov-15

Slogan: Prints colored with Velox transparent water color stamps

Subject(s): product - misc.

Product: Velox Transparent Water Color Stamps

Description / Notes: price for book: $\$ 0.25$. Price for complete kit: $\$ 0.75$

Binder: 1913-1917 Amateur Ads 3 of 4

Year: Aug-15

Slogan: The Vest Pocket Autographic Kodak

Subject(s): product - camera

Product: Vest Pocket Autographic Kodak

Description / Notes: price: $\$ 10.00$

Binder: 1913-1917 Amateur Ads 3 of 4

Year: Dec-15

Slogan: No photographic equipment is complete without a Vest Pocket Autographic Kodak

Subject(s): product - camera

Product: Vest Pocket Autographic Kodak

Description / Notes: price: $\$ 6.00$ or $\$ 10.00$ with an Anastigmat 
Camera.

Binder: 1913-1917 Amateur Ads 3 of 4

Year: May-16

Slogan: A notebook that takes pictures. A camera that takes

notes.

Subject(s): product - camera

Product: Vest Pocket Autographic Kodak

Description / Notes: Price: $\$ 10.00$

Binder: 1913-1917 Amateur Ads 4 of 4

Year: Sep-16

Slogan: If you don't know how to make good pictures this book will help you.

Subject(s): product - misc.

Product: "How to Make Good Pictures" booklet

Description / Notes: Price: $\$ 0.25$. Library edition, cloth bound: $\$ 1.00$

Binder: 1913-1917 Amateur Ads 4 of 4

Year: Dec-16

Slogan: The title of the book tells the story

Subject(s): product - misc.

Product: "How to Make Good Pictures" booklet

Description / Notes: Price: $\$ 0.25$. Price for cloth bound edition $\$ 1.00$

Binder: 1913-1917 Amateur Ads 4 of 4

Year: Mar-17

Slogan: It fits the pocket

Subject(s): product - camera

Product: 2c Kodak Jr.

Description / Notes: Price: from $\$ 12.00$ to $\$ 19.00$

Binder: 1913-1917 Amateur Ads 4 of 4

Year: Feb-17

Slogan: 3a Autographic Kodak Special

Subject(s): product - camera

Product: 3a Autographic Kodak Special
Description / Notes: price: $\$ 55.00$

Binder: 1913-1917 Amateur Ads 4 of 4

Year: May-17

Slogan: The New 3a Autographic Kodak Special with Kodak Range Finder

Subject(s): product - camera

Product: 3a Autographic Kodak Special

Description / Notes: Price: $\$ 55.00$ and $\$ 66.00$

Binder: 1913-1917 Amateur Ads 4 of 4

Year: Jan-17

Slogan: Artura Carbon Black are better enlargements because they retain the contact quality.

Subject(s): product - film \& paper

Product: Artura Carbon Black Paper

Color or Black and White:

Binder: 1913-1917 Amateur Ads 4 of 4

Year: Jun-16

Slogan: Artura it produces quality for quality

Subject(s): product - film \& paper

Product: Artura Paper

Color or Black and White:

Binder: 1913-1917 Amateur Ads 4 of 4

Year: Jul-16

Slogan: Artura retains this popularity because of its superior

quality

Subject(s): product - film \& paper

Product: Artura Paper

Color or Black and White:

Binder: 1913-1917 Amateur Ads 4 of 4

Year: Nov-16

Slogan: Your customers may not know why they prefer prints on

Artura

Subject(s): product - film \& paper

Product: Artura Paper 


\section{Color or Black and White:}

Binder: 1913-1917 Amateur Ads 4 of 4 Year: Feb-17

Slogan: Quality First.

Subject(s): product - film \& paper

Product: Artura Paper

Color or Black and White:

Binder: 1913-1917 Amateur Ads 4 of 4 Year: Oct-16

Slogan: Write it on the film--at the time.

Subject(s): product - camera

Product: Autographic Kodak

Description / Notes: "All folding Kodaks and Folding Brownies are now Autographic"

Binder: 1913-1917 Amateur Ads 4 of 4

Year: Nov-16

Slogan: Check up results with an Autographic Kodak

Subject(s): product - camera

Product: Autographic Kodak

Description / Notes: Article.

Binder: 1913-1917 Amateur Ads 4 of 4

Year: Apr-17

Slogan: Autographic, of course

Subject(s): product - camera

Product: Autographic Kodak

Description / Notes: article.

Binder: 1913-1917 Amateur Ads 4 of 4 Year: Nov-16

Slogan: Belt case for vest pocket Kodak

Subject(s): product - camera

Product: Belt case for Vest Pocket Kodak

Description / Notes: price: $\$ 1.50$

Binder: 1913-1917 Amateur Ads 4 of 4
Year: Jun-16

Slogan: It's as simple to make enlargements with a Brownie

Enlarging Camera as it is to make pictures with a Kodak

Subject(s): product - camera

Product: Brownie Enlarging Camera

Description / Notes: Price: from $\$ 1.75$ to $\$ 4.00$

Binder: 1913-1917 Amateur Ads 4 of 4

Year: Oct-16

Slogan: Large Prints with a Brownie Enlarging Camera

Subject(s): product - camera

Product: Brownie Enlarging Camera

Description / Notes: Article.

Binder: 1913-1917 Amateur Ads 4 of 4

Year: Jan-17

Slogan: As far as enlarging goes you can do without daylight with the Brownie Enlarging Camera

Subject(s): product - camera

Product: Brownie Enlarging Camera

Description / Notes: Price: $\$ 3.00$

Binder: 1913-1917 Amateur Ads 4 of 4

Year: Jul-16

Slogan: When you take a Kodak with you provide yourself with a carrying case.

Subject(s): product - camera

Product: Camera carrying case

Color or Black and White:

Binder: 1913-1917 Amateur Ads 4 of 4

Year: Oct-16

Slogan: Core Plate Developing Racks / Eastman Film Developing Holder

Subject(s): product - darkroom

Product: Core Plate Developing Racks / Eastman Film Developing Holder

Description / Notes: Core plate price: from $\$ 0.50$ to $\$ 1.60$.

Eastman film developing holder price: $\$ 0.40$ to $\$ 0.50$ 
Binder: 1913-1917 Amateur Ads 4 of 4

Year: Apr-17

Slogan: Eastman Film Developing Holder No. 2 / Core Plate Developing Racks

Subject(s): product - darkroom

Product: Core plate developing racks, Eastman film developing box No. 2.

Description / Notes: Price for Film holder: $\$ 0.30$ to $\$ 1.50$. Price for racks: $\$ 0.60$ to $\$ 1.60$

Binder: 1913-1917 Amateur Ads 4 of 4

Year: Nov-16

Slogan: Don't overlook the big profits of the enlarging business.

Subject(s): product - darkroom

Product: Eastman Enlarging Outfit

Description / Notes: Price for outfit with lamp, but without lens: $\$ 100.00$. Lens price: $\$ 12.00$

Binder: 1913-1917 Amateur Ads 4 of 4

Year: Jan-17

Slogan: Eastman Flash sheets and Kodak Flash Sheet Holder

Subject(s): product - misc.

Product: Eastman Flash Sheets

Description / Notes: price for flash sheet holder: $\$ 1.00$

Binder: 1913-1917 Amateur Ads 4 of 4

Year: Feb-17

Slogan: Fun "By Flashlight"

Subject(s): product - misc.

Product: Eastman Flash Sheets

Description / Notes: Article.

Binder: 1913-1917 Amateur Ads 4 of 4

Year: Oct-16

Slogan: This seal on the container guarantees the quality of its contents

Subject(s): product - Chemicals

Product: Eastman Kodak Company Tested Chemicals

\section{Color or Black and White}

Binder: 1913-1917 Amateur Ads 4 of 4

Year: Nov-16

Slogan: Tozol The Complete Developer

Subject(s): product - Chemicals

Product: Eastman Kodak Company Tested Chemicals

Description / Notes: Price: from $\$ 1.20$ to $\$ 18.00$

Binder: 1913-1917 Amateur Ads 4 of 4

Year: Dec-16

Slogan: Eastman Special Developer

Subject(s): product - Chemicals

Product: Eastman Kodak Company Tested Chemicals

Description / Notes: Price: $\$ 0.25$

Binder: 1913-1917 Amateur Ads 4 of 4

Year: Dec-16

Slogan: This seal outside the container means that the chemical

or chemical preparation inside is exactly what it should be--not

only Eastman tested but Eastman passed.

Subject(s): product - Chemicals

Product: Eastman Kodak Company Tested Chemicals

Color or Black and White:

Binder: 1913-1917 Amateur Ads 4 of 4

Year: Dec-16

Slogan: The New Developer: Kodelon

Subject(s): product - Chemicals

Product: Eastman Kodak Company Tested Chemicals

Description / Notes: Kodelon developer price: from \$0.89 to

59.50

Binder: 1913-1917 Amateur Ads 4 of 4

Year: Mar-17

Slogan: Eastman Tellurium Toner - It cleans and warms the print

Subject(s): product - Chemicals

Product: Eastman Kodak Company Tested Chemicals

Color or Black and White: 
Binder: 1913-1917 Amateur Ads 4 of 4

Year: Apr-17

Slogan: This seal is on every carton of Nepera Solution

Subject(s): product - Chemicals

Product: Eastman Kodak Company Tested Chemicals

Description / Notes: Price: $\$ 0.28$ to $\$ 0.84$

Binder: 1913-1917 Amateur Ads 4 of 4

Year: Apr-17

Slogan: Look for our stamp of approval on every container

Subject(s): product - Chemicals

Product: Eastman Kodak Company Tested Chemicals

Color or Black and White:

Binder: 1913-1917 Amateur Ads 4 of 4

Year: Jan-17

Slogan: What the Kodak Film Tank does for films, the Eastman Plate Tank does for plates

Subject(s): product - film \& paper

Product: Eastman Plate Tank

Description / Notes: Plate tank prices: $\$ 4.00$ to $\$ 5.00$

Binder: 1913-1917 Amateur Ads 4 of 4

Year: Mar-17

Slogan: Eastman Portrait Albums

Subject(s): product - misc.

Product: Eastman Portrait Albums

Description / Notes: Price: $\$ 10.00$

Binder: 1913-1917 Amateur Ads 4 of 4

Year: Jun-16

Slogan: Sparkle and Pluck have a special meaning when applied to Eastman Portrait Film

Subject(s): product - film \& paper

Product: Eastman Portrait Film

Color or Black and White:

Binder: 1913-1917 Amateur Ads 4 of 4
Year: Jul-16

Slogan: 93\% Increase

Subject(s): product - film \& paper

Product: Eastman Portrait Film

Color or Black and White:

Binder: 1913-1917 Amateur Ads 4 of 4

Year: Aug-16

Slogan: The Quality of Eastman Portrait Film

Subject(s): product - film \& paper

Product: Eastman Portrait Film

Color or Black and White:

Binder: 1913-1917 Amateur Ads 4 of 4

Year: Aug-16

Slogan: Eastman Studio Scale

Subject(s): product - misc.

Product: Eastman Studio Scale

Description / Notes: Price: $\$ 3.00$

Binder: 1913-1917 Amateur Ads 4 of 4

Year: Nov-16

Slogan: The good points of the Eastman Trimmer

Subject(s): product - misc.

Product: Eastman Trimmer / R.O.C. Trimmer

Description / Notes: Price for Eastman Trimmer: $\$ 6.00$ to

$\$ 12.00$. Price for R.O.C. Trimmer: $\$ 1.50$ to $\$ 2.75$

Binder: 1913-1917 Amateur Ads 4 of 4

Year: Jan-17

Slogan: A New Size that Fits. Eastman View Camera No. 2

Subject(s): product - camera

Product: Eastman View Camera No. 2.

Description / Notes: Price: $\$ 35.00$

Binder: 1913-1917 Amateur Ads 4 of 4

Year: Dec-16

Slogan: There's a Merry Christmas for the Amateur in any one of these Kodak helps. 
Subject(s): product - misc.

Product: Enlarging Cameras, tripod, albums, flashlight, Safelight

Description / Notes: Gift ideas.

Binder: 1913-1917 Amateur Ads 4 of 4

Year: May-17

Slogan: Wratten Filters / Orthochromatic Filters / Contrast Filters Subject(s): product - misc.

Product: Filters

Description / Notes: Prices: from $\$ 0.25$ to $\$ 6.25$

Binder: 1913-1917 Amateur Ads 4 of 4

Year: Nov-16

Slogan: Flexo Print Roller / Eastman Photo Paste

Subject(s): product - misc.

Product: Flexo Print Roller / Eastman Photo Paste

Description / Notes: price for flexo print roller: $\$ 0.20$ price for photo paste: from $\$ 0.10$ to $\$ 0.25$

Binder: 1913-1917 Amateur Ads 4 of 4

Year: Sep-16

Slogan: If it's a Folding Brownie or a Folding Kodak, it's

Autographic.

Subject(s): product - camera

Product: Folding Kodaks and Folding Brownies

Description / Notes: Article.

Binder: 1913-1917 Amateur Ads 4 of 4

Year: Jul-16

Slogan: Keep the summer story told by your Kodak in book form with a Hercules Album

Subject(s): product - misc.

Product: Hercules Album

Description / Notes: Price: $\$ 3.25$ to $\$ 5.00$

Binder: 1913-1917 Amateur Ads 4 of 4

Year: Jun-17

Slogan: Kodak, as you go

Subject(s): Maid
Text: none

Implied Audience: Women

Product: Kodak

Photo or Illustration Based: photo

Color or Black and White: color

Publications: Outlook

Description / Notes: Color ad of the maid giving the camera to the woman in the car. The ad comes with an Outlook magazine,

dated June 1917. Also, a letter to Mr. Jack Buggington, Inverness

Florida dated August 1975, from Glenn W. Mentch, Director,

Advertising Services. (it appears he had sent this ad to the

department, and it was added to this binder.)

Binder: 1913-1917 Amateur Ads 4 of 4

Year: Oct-16

Slogan: Two Kodak Helps: Kodak Maskit Printing Frame, Eastman Visible Graduate

Subject(s): product - darkroom

Product: Kodak "Maskit" printing frame, Eastman Visible Graduate

Description / Notes: price for printing frame: $\$ 0.40$ to $\$ 0.50$.

Price for Graduate: $\$ 0.20$ to $\$ 0.60$

Binder: 1913-1917 Amateur Ads 4 of 4

Year: Dec-16

Slogan: Holiday Greeting Carriers.

Subject(s): product - misc.

Product: Kodak accessories

Description / Notes: Article about gift ideas.

Binder: 1913-1917 Amateur Ads 4 of 4

Year: Oct-16

Slogan: The Kodak Amateur Printer

Subject(s): product - darkroom

Product: Kodak Amateur Printer

Description / Notes: Price: $\$ 6.00$

Binder: 1913-1917 Amateur Ads 4 of 4

Year: Aug-16

Slogan: The experience is in the tank. The efficiency is in the 
printer.

Subject(s): product - darkroom

Product: Kodak Amateur Printer / Kodak Film Tank

Description / Notes: Price for printer: $\$ 6.00$. Price for Film Tank: $\$ 2.75$

Binder: 1913-1917 Amateur Ads 4 of 4

Year: Dec-16

Slogan: Kodak Film Tank and the Kodak Amateur Printer

Subject(s): product - darkroom

Product: Kodak Amateur Printer / Kodak Film Tank

Color or Black and White:

Binder: 1913-1917 Amateur Ads 4 of 4

Year: Jan-17

Slogan: The Newest of the Kodak Helps

Subject(s): product - darkroom

Product: Kodak Auto-Mask Printing Frame

Description / Notes: Price: $\$ 1.00$

Binder: 1913-1917 Amateur Ads 4 of 4

Year: Feb-17

Slogan: Kodak Color Filter

Subject(s): product - camera

Product: Kodak color filter

Description / Notes: Price from $\$ 0.50$ to $\$ 1.00$

Binder: 1913-1917 Amateur Ads 4 of 4

Year: May-17

Slogan: Kodak Dry Mounting Press

Subject(s): product - misc.

Product: Kodak Dry Mounting Press

Description / Notes: Price: $\$ 15.00$ to $\$ 57.00$

Binder: 1913-1917 Amateur Ads 4 of 4

Year: Jul-16

Slogan: A little over twenty minutes after the last exposure has been made, you will be able to see "how they came out" with a Kodak Film Tank
Subject(s): product - darkroom

Product: Kodak Film Tank

Description / Notes: Price from \$2.75 up.

Binder: 1913-1917 Amateur Ads 4 of 4

Year: Sep-16

Slogan: Developing is done in daylight with a Kodak Film Tank. And the Experience is in the tank.

Subject(s): product - darkroom

Product: Kodak Film Tank

Description / Notes: Price: $\$ 2.75$ to $\$ 8.00$

Binder: 1913-1917 Amateur Ads 4 of 4

Year: Jan-17

Slogan: It Does the Rest

Subject(s): product - darkroom

Product: Kodak Film Tank

Description / Notes: Article.

Binder: 1913-1917 Amateur Ads 4 of 4

Year: Oct-16

Slogan: Kodak Flash Sheet Holder

Subject(s): product - misc.

Product: Kodak flash sheet holder

Description / Notes: Price: $\$ 1.00$

Binder: 1913-1917 Amateur Ads 4 of 4

Year: Mar-17

Slogan: In-Door Photography

Subject(s): product - misc.

Product: Kodak flash sheet holder, Portrait attachment

Description / Notes: Article.

Binder: 1913-1917 Amateur Ads 4 of 4

Year: Aug-16

Slogan: Full tripod service means a Kodak Metal Tripod

Subject(s): product - misc.

Product: Kodak Metal tripod

Description / Notes: Price: from \$2.25 to \$6.00. Leather case: 
$\$ 1.75$

Binder: 1913-1917 Amateur Ads 4 of 4 Year: Nov-16

Slogan: Make the most of the Thanksgiving homecoming with a Kodak and Kodak Portrait Attachment

Subject(s): product - camera

Product: Kodak Portrait Attachment

Description / Notes: Price: $\$ 0.50$

Binder: 1913-1917 Amateur Ads 4 of 4

Year: May-17

Slogan: The Kodak Range Finder

Subject(s): product - camera

Product: Kodak Range Finder

Description / Notes: article.

Binder: 1913-1917 Amateur Ads 4 of 4

Year: Jul-16

Slogan: Two methods of dark room illumination that you can be sure are safe.

Subject(s): product - darkroom

Product: Kodak safelight lamp, Brownie safelight lamp.

Description / Notes: Price for Kodak lamp: \$3.00. Price for Brownie lamp: \$1.00

Binder: 1913-1917 Amateur Ads 4 of 4

Year: Jul-16

Slogan: Finish as you Take

Subject(s): product - darkroom

Product: Kodak System

Description / Notes: Article.

Binder: 1913-1917 Amateur Ads 4 of 4

Year: Sep-16

Slogan: This is the open season for Kodak Velvet Green Paper

Subject(s): product - film \& paper

Product: Kodak Velvet Green Paper

Color or Black and White:
Binder: 1913-1917 Amateur Ads 4 of 4

Year: Apr-17

Slogan: And now for prints on Kodak Velvet Green

Subject(s): product - film \& paper

Product: Kodak Velvet Green Paper

Color or Black and White

Binder: 1913-1917 Amateur Ads 4 of 4

Year: Dec-16

Slogan: Kodak

Subject(s): Woman

Text: Give your Christmas shopping the benefit of your

experience. Kodak has meant endless pleasure to you--it can

mean as much to anyone on your Christmas list. It's the logical gift for you to make.

Implied Audience: women / Christmas

Product: Kodaks and Brownies

Photo or Illustration Based: photo

Color or Black and White: bw

Description / Notes: Photo of a woman sitting in a parlor chair, holding a Kodak, presents in the BG on a table.

Binder: 1913-1917 Amateur Ads 4 of 4

Year: Jun-16

Slogan: Here is true Kodak efficiency

Subject(s): product - camera

Product: No. 1 Autographic Kodak, Special

Description / Notes: Price: $\$ 40.00$

Binder: 1913-1917 Amateur Ads 4 of 4

Year: Aug-16

Slogan: The New Size Picture

Subject(s): product - camera

Product: No. 2c Folding Autographic Brownie

Description / Notes: Article.

Binder: 1913-1917 Amateur Ads 4 of 4

Year: Aug-16 
Slogan: The No. 3a Folding Autographic Brownie

Subject(s): product - camera

Product: No. 3a Folding Autographic Brownie

Description / Notes: price: $\$ 10.00$ and $\$ 12.00$

Binder: 1913-1917 Amateur Ads 4 of 4

Year: May-17

Slogan: There's room for the rest of the view with the Panoram Kodak

Subject(s): product - camera

Product: Panoram Kodak No.1 and No. 4

Description / Notes: No. 1 price: $\$ 10.00$. No. 4 Price: $\$ 20.00$

Binder: 1913-1917 Amateur Ads 4 of 4

Year: Jun-16

Slogan: The 1916 Kodak Advertising Competition

Subject(s): product - misc.

Product: Photo contest

Description / Notes: Article.

Binder: 1913-1917 Amateur Ads 4 of 4

Year: Jul-16

Slogan: 1916 Kodak Advertising Competition

Subject(s): product - misc.

Product: Photo contest

Description / Notes: "For the best of such pictures, ten prizes are offered ranging from $\$ 100.00$ to $\$ 1,000.00 "$

Binder: 1913-1917 Amateur Ads 4 of 4

Year: Sep-16

Slogan: None

Subject(s): product - misc.

Product: Photo contest

Description / Notes: Ad for the yearly photo contest with a photo of last years winner (no name given) "A Selling argument put in a print--that's what we want" Photo is of two women on the beach photographing their children. (am sure that was actually used in an ad.)
Binder: 1913-1917 Amateur Ads 4 of 4

Year: May-17

Slogan: 1917 Kodak Advertising Competition

Subject(s): product - misc.

Product: Photo contest

Description / Notes: "In which prizes aggregating 3,000.00 are to be given."

Binder: 1913-1917 Amateur Ads 4 of 4

Year: Nov-16

Slogan: A reputation for promptness is a big asset, especially in a busy season--save time by using a Rounds Print Washer /

Improved Majestic Print Dryer.

Subject(s): product - darkroom

Product: Rounds print washer, Improved majestic print dryer

Description / Notes: Price for washer: $\$ 12.00$ and $\$ 33.00$. Price for No. 1 dryer: $\$ 25.00$

Binder: 1913-1917 Amateur Ads 4 of 4

Year: May-17

Slogan: Prints on Royal Velox

Subject(s): product - film \& paper

Product: Royal Velox

Color or Black and White:

Binder: 1913-1917 Amateur Ads 4 of 4

Year: Jul-16

Slogan: Seed plates

Subject(s): product - film \& paper

Product: Seed Dry Plates

Color or Black and White:

Binder: 1913-1917 Amateur Ads 4 of 4

Year: Dec-16

Slogan: Seed Plates. All the quality you look for in a plate--all the time.

Subject(s): product - film \& paper

Product: Seed Dry Plates

Color or Black and White 
Binder: 1913-1917 Amateur Ads 4 of 4

Year: Nov-16

Slogan: System for the Studio

Subject(s): product - misc.

Product: Studio Cash Book, Shop tickets, Follow Up Cards, and Studio Register

Description / Notes: Price for cash book: \$4.00. Price for 100

shop tickets: $\$ 0.25$. Price for follow-up cards: $\$ 0.60$. Price for

Studio Register: $\$ 5.00$

Binder: $1913-1917$ Amateur Ads 4 of 4

Year: Oct-16

Slogan: Kodak Told the Story, let a Kodak Album Keep it.

Subject(s): product - film \& paper

Product: The Kodak Album, The Hercules Album

Description / Notes: Price for Kodak Album: $\$ 2.25$ or $\$ 3.50$

Price for Hercules Album: $\$ 2.65$ to $\$ 5.00$

Binder: 1913-1917 Amateur Ads 4 of 4

Year: Nov-16

Slogan: The Kodak Film Tank makes anybody an expert as far as

film developing goes. All by daylight.

Subject(s): product - darkroom

Product: The Kodak Film Tank

Description / Notes: price: from $\$ 2.75$ to $\$ 8.00$

Binder: 1913-1917 Amateur Ads 4 of 4

Year: Aug-16

Slogan: New even to the size of the picture it makes

Subject(s): product - camera

Product: The No. 2C Autographic Kodak Junior

Description / Notes: Price: from $\$ 12.00$ to $\$ 19.00$

Binder: 1913-1917 Amateur Ads 4 of 4

Year: Nov-16

Slogan: The Olympic Calendar

Subject(s): product - misc.

Product: The Olympic Calendar

\section{Color or Black and White}

Binder: 1913-1917 Amateur Ads 4 of 4

Year: Dec-16

Slogan: Handsome in itself. The Olympic Calendar

Subject(s): product - misc.

Product: The Olympic Calendar

Color or Black and White:

Binder: 1913-1917 Amateur Ads 4 of 4

Year: Sep-16

Slogan: A novel effect and you'll like it.

Subject(s): product - misc.

Product: The Woodmat, The souvenir photo case

Description / Notes: price for woodmat: $\$ 0.05$ to $\$ 0.10$. Price

for Souvenir photo case: $\$ 0.35$ to $\$ 0.50$

Binder: 1913-1917 Amateur Ads 4 of 4

Year: Aug-16

Slogan: Universal Tripod Head

Subject(s): product - misc.

Product: Universal tripod head

Description / Notes: Price: $\$ 0.75$

Binder: 1913-1917 Amateur Ads 4 of 4

Year: Jun-16

Slogan: The best finisher uses Velox

Subject(s): product - film \& paper

Product: Velox paper

Color or Black and White:

Binder: 1913-1917 Amateur Ads 4 of 4

Year: Aug-16

Slogan: You're entitled to Velox

Subject(s): product - film \& paper

Product: Velox paper

Color or Black and White:

Binder: 1913-1917 Amateur Ads 4 of 4 
Year: Aug-16

Slogan: Kodak Eastman Film Velox

Subject(s): product - film \& paper

Product: Velox paper

Color or Black and White:

Binder: 1913-1917 Amateur Ads 4 of 4

Year: Oct-16

Slogan: If it isn't Velox it isn't the amateur's own paper

Subject(s): product - film \& paper

Product: Velox paper

Color or Black and White:

Binder: 1913-1917 Amateur Ads 4 of 4

Year: Dec-16

Slogan: Re-development with Royal Velox

Subject(s): product - film \& paper

Product: Velox paper

Color or Black and White:

Binder: 1913-1917 Amateur Ads 4 of 4

Year: Apr-17

Slogan: The Best Print you can get on Velox is the best print you can get

Subject(s): product - film \& paper

Product: Velox paper

Color or Black and White:

Binder: 1913-1917 Amateur Ads 4 of 4

Year: Aug-16

Slogan: Color your vacation prints with Velox Transparent Water Color Stamps

Subject(s): product - film \& paper

Product: Velox Transparent Water Color Stamps

Description / Notes: price for book of stamps: $\$ 0.25$. Price for complete outfit: $\$ 0.75$

Binder: 1913-1917 Amateur Ads 4 of 4

Year: Jan-17
Slogan: This is the actual size of the Vest Pocket Autographic Kodak

Subject(s): product - camera

Product: Vest Pocket Autographic Kodak

Description / Notes: Vest Pocket Autographic Kodak price: from $\$ 6.00$ to $\$ 22.50$

Binder: 1913-1917 Amateur Ads 4 of 4

Year: Apr-17

Slogan: Vest Pocket Autographic Kodak Special

Subject(s): product - camera

Product: Vest Pocket Autographic Kodak Special

Description / Notes: Price from $\$ 10.00$ to $\$ 22.50$

Binder: 1913-1917 Amateur Ads 4 of 4

Year: Apr-17

Slogan: Vest Pocket Kodak Tripod Adapter

Subject(s): product - camera

Product: Vest Pocket Kodak Tripod Adapter

Description / Notes: Price: $\$ 0.50$

Binder: 1913-1917 Amateur Ads 4 of 4

Year: Jul-16

Slogan: The Wratten Safelight Lamp

Subject(s): product - darkroom

Product: Wratten Safelight Lamp

Description / Notes: price: from $\$ 6.00$ to $\$ 9.00$

Binder: 1913-1917 Amateur Ads 4 of 4

Year: Oct-16

Slogan: Wratten Safelight Lamps / Kodak Safelight Lamp

Subject(s): product - darkroom

Product: Wratten Safelight Lamp / Kodak Safelight Lamp

Description / Notes: Price for Wratten: \$9.00. Price for Kodak: $\$ 3.00$

Binder: $1914-19161$ of 5

Year: 1914

Slogan: The 2a Folding Pocket Brownie 
Subject(s): product - camera

Product: 2a Folding Pocket Brownie

Description / Notes: Price: $\$ 6.00$

Binder: $1914-19161$ of 5

Slogan: Write the Date on the negative

Subject(s): Kodak Girl

Text: Make the pictures that you take doubly valuable by

recording, briefly, on the margin of the film negative the all

important: who - when - where. You can do it instantly,

permanently, at the time you make the exposure with an

autographic Kodak.

Implied Audience: women / genera

Product: autographic Kodak

Photo or Illustration Based: photo

Color or Black and White: bw

Description / Notes: photo of a woman outdoors making a note on the film.

Binder: 1914-1916 1 of 5

Slogan: Use an Autographic Kodak

Subject(s): man on construction site.

Text: Every negative that is worth making is worth a date and

title. Architects, engineers, and contractors who make

photographic records of progressive work and the amateur who

wants to improve the quality of his work can make valuable

notations on the negatives, by means of the autographic Kodak.

The places visited - interesting dates and facts, such notations add

to the value of every negative. The greatest photographic advance

in 20 years.

Implied Audience: Laborers

Product: autographic Kodak

Photo or Illustration Based: photo

Color or Black and White: bw

Description / Notes: photograph of a man at a construction site using the autographic Kodak.

Binder: $1914-19161$ of 5

Slogan: Make Sure -
Subject(s): man writing on autographic camera

Text: Write the date and title on the film at the time you make the exposure. Add to the value of every negative with a permanent

record that you can always have for reference. It's only a matter of seconds with an Autographic Kodak..

Implied Audience: outdoors

Product: autographic Kodak

Photo or Illustration Based: photo

Color or Black and White: bw

Description / Notes: photo of a man in a yard writing with the autographic camera.

Binder: 1914-1916 1 of 5

Slogan: Write it on the film - at the time.

Subject(s): mother / child

Text: Make your Kodak story of the children doubly valuable, by dating every negative, by making brief notes that will help, in after years, to recall happily to memory the incident that led to the taking of the picture. Dressed for her first party. The first school day. Bob's first skates. Mary's fourth birthday. - a thousand such important events should be titled on the negatives and at leisure copied into the Kodak album. It's a simple almost instantaneous process with an Autographic Kodak.

Implied Audience: families

Product: autographic Kodak

Photo or Illustration Based: photo

Color or Black and White: bw

Description / Notes: Photograph of a mother writing on the Kodak, while the young daughter watches.

Binder: $1914-19161$ of 5

Slogan: Write it on the film - at the time.

Subject(s): Woman

Text: Make every negative more valuable by permanently

recording at the time of exposure the all important date and title. It's a simple and almost instantaneous process with an

Autographic Kodak

Implied Audience: women / general

Product: autographic Kodak 
Photo or Illustration Based: photo

Color or Black and White: bw

Description / Notes: photograph of a woman inscribing autographic Kodak.

Binder: 1914-1916 1 of 5

Year: 1914

Slogan: Better than any mere toy.

Subject(s): product - camera

Product: Brownie Camera

Description / Notes: Price: $\$ 1.00$ to $\$ 12.00$

Binder: 1914-1916 1 of 5

Year: 1914

Slogan: Eastman Dental X-Ray Films

Subject(s): product - medical

Product: Eastman Dental X-Ray Films

Color or Black and White:

Binder: $1914-19161$ of 5

Year: 1914

Slogan: A Clear Picture

Subject(s): product - film \& paper

Product: Eastman Film

Color or Black and White:

Binder: $1914-19161$ of 5

Year: 1914

Slogan: No Matter How Thrilling

Subject(s): product - film \& paper

Product: Eastman Film

Color or Black and White:

Binder: 1914-1916 1 of 5

Year: 1914

Slogan: "How Clear the Pictures Are!"

Subject(s): product - film \& paper

Product: Eastman Film

Color or Black and White:
Binder: $1914-19161$ of 5

Year: 1914

Slogan: Serial Stomach Examinations

Subject(s): product - medical

Product: Eastman Screen X-Ray film

Color or Black and White:

Binder: 1914-1916 1 of 5

Year: 1914

Slogan: X-Ray Films

Subject(s): product - medical

Product: Eastman X-Ray Film

Color or Black and White:

Binder: $1914-19161$ of 5

Year: 1914

Slogan: X-Ray Efficiency

Subject(s): product - medical

Product: Eastman X-Ray Film

Color or Black and White:

Binder: $1914-19161$ of 5

Year: 1914

Slogan: Eastman X-Ray Films

Subject(s): product - medical

Product: Eastman X-Ray Film

Color or Black and White:

Binder: 1914-1916 1 of 5

Year: 1914

Slogan: For Out of Town Work

Subject(s): product - medical

Product: Eastman X-Ray Film

Color or Black and White:

Binder: $1914-19161$ of 5

Year: 1914

Slogan: The New X-Ray Film 
Subject(s): product - medical Product: Eastman X-Ray Film

Color or Black and White:

Binder: $1914-19161$ of 5

Year: 1914

Slogan: Eastman Screen X-Ray Film

Subject(s): product - medical

Product: Eastman X-Ray Film

Color or Black and White:

Binder: 1914-1916 1 of 5

Slogan: The Kodak on the Farm

Subject(s): Chicken and chicks

Text: every live thing on the farm is worth a photograph - and just now when chicks and lambs and calves are arriving so rapidly is a most interesting time to start a Kodak record - such a record often becomes more then interesting, it becomes a business asset. ...

Implied Audience: farmers

Product: Kodak

Photo or Illustration Based: illus

Color or Black and White: bw

Description / Notes: simple illustration

Binder: $1914-19161$ of 5

Slogan: Kodak on the Farm

Subject(s): cows

Text: At every turn, country life offers opportunity for the camera.

In a business way there are records of stock and crops and

buildings and trees and ditching to be kept. From the standpoint of the family album there are pictures of the children and pets and

the home - pictures that grow in interest with every passing year. And the Kodak adds fun to every wholesome frolic. At the picnic,

at every evening gathering, on the trip to the city, on the shooting and fishing trips it adds to the pleasure at the time and the resulting pictures are a pleasure for all time. And the cost is less than you think.

Implied Audience: farmers

Product: Kodak
Photo or Illustration Based: illustration

Color or Black and White: bw

Description / Notes: illustration of hands photographing a herd of cows.

Binder: $1914-19161$ of 5

Slogan: All Out-doors invites your Kodak

Subject(s): farmer / wife standing with corn

Text: Everything that you are interested in is worth a picture. On

the home place, at your neighbor's, at the picnic, on your fishing

or hunting trip - whenever you go there are scenes and incidents

that you can preserve for all time with the click of a Kodak shutter.

Implied Audience: farmers

Product: Kodak

Photo or Illustration Based: illus

Color or Black and White: bw

Description / Notes: another simple illustration for the Kodak on the Farm series.

Binder: $1914-19161$ of 5

Slogan: For the boy - A Kodak

Subject(s): father / son at camera store

Text: Indoors and out he will enjoy the making of pictures. 'Twill mean more fun on the vacation and the after-pleasure of showing

the pictures to his chums.

Implied Audience: families

Product: Kodak

Photo or Illustration Based: photo

Color or Black and White: bw

Description / Notes: Photograph of a father and son in a camera store. The son is holding a camera as the father and salesman look on.

Binder: 1914-1916 1 of 5

Slogan: Keep a Kodak Baby Book

Subject(s): Father photographing mother and baby.

Text: The first journey downstairs for exhibition to that secondary consideration - father. The toddling nursery days! That all 
important epoch when the baby first trudges off to school. In all these great events are limitless opportunities for the Kodak. And with the school days come pictures by, as well as pictures of the children. Pictures they take of each other, free from constraint or conscious posing. Spontaneous pictures that reflect simplicity and weave into the Kodak Book the touch of naturalness. It is such pictures as these that add the delightful side-lights to the more formal studio pictures that must be made of little John and Mary. Just as they must have 'dress up clothes' for Sunday School and parties, so, too, you will want them in 'dress up pictures.' But you love them as much in their soiled pinafores as in their party best. And, too, you will love the Kodak pictures that hold the charm of homeyness.

Implied Audience: families

Product: Kodak

Photo or Illustration Based: photo

Color or Black and White: bw

Description / Notes: Photograph of a father with camera and

tripod photographing mother and baby - both in white.

Binder: 1914-1916 1 of 5

Slogan: All Out-doors invites your Kodak

Subject(s): Kodak Girl

Text: none

Implied Audience: women

Product: Kodak

Photo or Illustration Based: photo

Color or Black and White: bw

Description / Notes: Photograph of a woman leaning on a fence, at the lakeshore composing a photograph.

Binder: 1914-1916 1 of 5

Slogan: For Christmas Morning A Kodak

Subject(s): Kodak Girl

Text: And throughout the day, the taking of pictures of all that

goes to make that day a merry one.

Implied Audience: women / Christmas

Product: Kodak

Photo or Illustration Based: photo
Color or Black and White: bw

Description / Notes: photo is a woman with a new Kodak sitting at a snowy window, with wrapped presents.

Binder: 1914-1916 1 of 5

Slogan: Anywhere - Everywhere Kodak

Subject(s): Kodak girl in silhouette

Text: Indoors or out, on your travels or at home, Kodak is at your service. And it means photography with the bother left out.

Implied Audience: women / general

Product: Kodak

Photo or Illustration Based: photo

Color or Black and White: bw

Description / Notes: Silhouette of a woman holding a Kodak.

The caption asks the reader to read the March issue of Kodakery to find out how to make these silhouette photographs.

Binder: 1914-1916 1 of 5

Slogan: Take a Kodak with You

Subject(s): man at a train station

Text: none

Implied Audience: trave

Product: Kodak

Photo or Illustration Based: photo

Color or Black and White: bw

Description / Notes: Photograph of a man sitting on his suitcase waiting for a train with Kodak slung over his shoulder.

Binder: $1914-19161$ of 5

Slogan: All Out-doors invites your Kodak

Subject(s): man, woman outdoors with golf clubs

Text: none

Implied Audience: trave

Product: Kodak

Photo or Illustration Based: photo

Color or Black and White: bw

Description / Notes: photograph of a young couple sitting on a hill with golf clubs, the woman is holding a Kodak. 
Binder: $1914-19161$ of 5

Year: 1914

Slogan: Keep a Kodak Record

Subject(s): men in orchard

Text: The photograph of the young orchard - just as it starts the summer growth - then another photograph, say in October when the growth is ended the wood is hardening, will make an interesting and valuable record. Such pictures become a real asset when used to comparative growths of trees of crops that have had different treatments in fertilizers or in cultivation. And picture making is very simple by the Kodak method, and less expensive than you think. Ask your dealer or write us for our new booklet "The Kodak on the Farm"

Implied Audience: farmers

Product: Kodak

Photo or Illustration Based: illus

Color or Black and White: bw

Description / Notes: simple illustration

Binder: $1914-19161$ of 5

Slogan: Let the Children Kodak

Subject(s): three young girls

Text: Twill be fun for them, will add to the joy of their out-door days. And afterward the unposed pictures of the children by the children - pictures free from consciousness and constraint - will double the value of the family Kodak album.

Implied Audience: families

Product: Kodak

Photo or Illustration Based: photo

Color or Black and White: bw

Description / Notes: photograph of three girls on a beach - two sitting under an umbrella - one photographing them.

Binder: 1914-1916 1 of 5

Slogan: All Out-doors invites your Kodak

Subject(s): young boy standing at lake edge

Text: none

Implied Audience: parents/children

Product: Kodak
Photo or Illustration Based: photo

Color or Black and White: bw

Description / Notes: Photograph of a boy looking out on a lake, with Kodak in hand.

Binder: 1914-1916 1 of 5

Slogan: Take a Kodak with You

Subject(s): young girl and boy

Text: Get more fun from every trip by taking pictures of all the

interesting people and places and 'doings'. And then you can enjoy the fun all over again in the Kodak album.

Implied Audience: parents/children

Product: Kodak

Photo or Illustration Based: photo

Color or Black and White: bw

Description / Notes: Photograph of a young boy and girl, boy holding suitcase, standing at a doorway. Girl with Kodak case

slung over her shoulder.

Binder: $1914-19161$ of 5

Slogan: The Story of the Kodak Album

Subject(s): family looking at album

Text: It's the intimate, personal story of the home - a picture story that interests every member of the family. And the older it grows, the more it expands, the stronger its grip becomes; the greater its fascination

Implied Audience: families

Product: Kodak album

Photo or Illustration Based: photo

Color or Black and White: bw

Description / Notes: Photograph of father, mother, three

children looking at the Kodak album. (could be grandparents.)

Binder: $1914-19161$ of 5

Year: 1915

Slogan: Keep a Kodak Baby Book

Subject(s): mother / child

Text: The first journey downstairs for exhibition to that secondary consideration - father. The toddling nursery days! That all 
important epoch when the baby first trudges off to school. In all these great events are limitless opportunities for the Kodak. And with the school days come pictures by, as well as pictures of the children. Pictures they take of each other, free from constraint or conscious posing. Spontaneous pictures that reflect simplicity and weave into the Kodak Book the touch of naturalness. It is such pictures as these that add the delightful side-lights to the more formal studio pictures that must be made of little John and Mary. Just as they must have 'dress up clothes' for Sunday School and parties, so, too, you will want them in 'dress up pictures.' But you love them as much in their soiled pinafores as in their party best. And, too, you will love the Kodak pictures that hold the charm of homeyness.

Implied Audience: families

Product: Kodak baby book

Photo or Illustration Based: photo

Color or Black and White: bw

Description / Notes: Photograph of a mother with tripod and camera, photographing baby on a wicker basket. Both are all in white.

Binder: 1914-1916 1 of 5

Slogan: If it isn't an Eastman it isn't a Kodak

Subject(s): product - misc.

Product: Kodak Brand

Description / Notes: about the Kodak brand, and warning against knock-offs.

Binder: $1914-19161$ of 5

Year: 1914

Slogan: Let Kodak Shorten the Winter Evenings

Subject(s): product - camera

Product: Kodak flash sheet holder

Color or Black and White:

Binder: $1914-19161$ of 5

Year: 1914

Slogan: The New Kodak Jr.

Subject(s): product - camera
Product: Kodak Jr.

Description / Notes: Price: from $\$ 7.50$ to $\$ 11.00$

Binder: $1914-19161$ of 5

Year: 1914

Slogan: No. 0 Brownie

Subject(s): product - camera

Product: No. 0 Brownie

Description / Notes: Price: $\$ 1.25$

Binder: $1914-19161$ of 5

Year: 1914

Slogan: No. 0 Brownie, The little camera that does big things

Subject(s): product - camera

Product: No. 0 Brownie

Description / Notes: Price: $\$ 1.25$

Binder: $1914-19161$ of 5

Year: 1914

Slogan: The No. 1 Autographic Kodak, Junior

Subject(s): product - camera

Product: No. 1 Autographic Kodak, Junior

Description / Notes: Price: $\$ 15.00$

Binder: $1914-19161$ of 5

Year: 1914

Slogan: The No. 1 Autographic Kodak, Special

Subject(s): product - camera

Product: No. 1 Autographic Kodak, Special

Description / Notes: Price: From $\$ 36.00$ to $\$ 56.00$

Binder: $1914-19161$ of 5

Year: 1914

Slogan: The Autographic Kodaks

Subject(s): product - camera

Product: No. 3a Autographic Kodak

Description / Notes: Price: $\$ 22.50$

Binder: 1914-1916 1 of 5 
Slogan: There's a photographer in your town

Subject(s): none

Text: Custom dictates that, as compared with Christmas, the

Easter gift shall be but a trifle - yet it shall convey the true spirit of the season - the spirit of goodwill and friendship and kindliness.

And when to these it adds the personal touch it conveys sentiment

without sentimentality. Between friends - a photograph.

Implied Audience: Easter / general

Product: photographs

Photo or Illustration Based: Text: Color or Black and White: bw

Description / Notes: the third ad selling photographs without using any visual aid.

Binder: $1914-19161$ of 5

Year: April, 1915

Slogan: There's a photographer in your town

Subject(s): none

Text: The simple gift that lends the touch of friendship without the embarrassment of an obligation - your photograph.

Product: photographs

Photo or Illustration Based: Text: Color or Black and White: bw

Description / Notes: no photograph - but selling a photograph!

Binder: 1914-1916 1 of 5

Slogan: There's a photographer in your town

Subject(s): none

Text: There's a quaint and pretty Indian superstition that those who are photographed lose something of their personality - that this personality becomes part of the picture. Be this superstition of be is sentiment, the idea bears both truth and charm. For a picture means something, is personal, gives the homely touch of

friendship--especially at Christmas time.

Product: photographs

Photo or Illustration Based: Text: Color or Black and White: bw

Description / Notes: no photograph - taking a superstition and making it an ad tactic!
Binder: $1914-19161$ of 5

Slogan: The Absentee

Subject(s): nothing!

Text: It is the day of all the year - thanksgiving day - when every member of the family is under the home roof-tree. Father is

skillfully disjointing the juicy gobbler, and mother, with anxiety lest the meal shall not go well sits opposite, serving the cranberries and supervising the whole ceremony; little Johnny is attacking a mighty drum stick and -and Oh! but the marmalade is good.

Thanksgiving Day, the family day, but with nearly always a regret that this one or that one could not be present. Had to go to Mary's folks this year, you know. Of course, it doesn't really take the place of the absentee, but on such occasions, along with the letter of regret - a new photograph. There's a photographer in your town.

Implied Audience: families

Product: photographs

Photo or Illustration Based: Text: Color or Black and White: bw

Description / Notes: clever - there's no photo, and it's called the absentee- emphasizing the lack of a photograph.

Binder: 1914-1916 1 of 5

Year: 1914

Slogan: Seed X-Ray Plates

Subject(s): product - medical

Product: Seed X-Ray Plates

Color or Black and White:

Binder: 1914-1916 1 of 5

Year: 1914

Slogan: In Stomach Cases

Subject(s): product - medical

Product: Seed X-Ray Plates

Color or Black and White:

Binder: 1914-1916 1 of 5

Year: 1914 
Slogan: As right as a full jeweled watch. Vest Pocket Kodak

Subject(s): product - camera

Product: Vest Pocket Kodak

Description / Notes: Price: $\$ 12.00$

Binder: $1914-19162$ of 5

Year: 1915

Slogan: The 3a Kodak

Subject(s): product - camera

Product: 3a Kodak

Description / Notes: Price: $\$ 27.50, \$ 22.50$

Binder: 1914-1916 2 of 5

Slogan: Make a Systematic record with an Autographic Kodak

Subject(s): man with camera, survey equipment

Text: none

Implied Audience: Laborers

Product: autographic Kodak

Photo or Illustration Based: photo

Color or Black and White: bw

Description / Notes: Photograph of a man in dirty work clothes, writing on a Kodak with survey equipment on a tripod.

Binder: $1914-19162$ of 5

Slogan: Write it on the film - at the time.

Subject(s): parents and baby

Text: Make the Kodak record accurate, authentic. Then there will never bee the question: "How old was Baby when this was taken," or "What summer was this made?" You can write the who, when and where permanently on the margin of the negative at the time the exposure is made if you use an Autographic Kodak. It's but a matter of a few seconds at the time and it's there to stay. All folding Kodaks now have the autographic feature and there is no extra charge for autographic film.

Implied Audience: families

Product: autographic Kodak

Photo or Illustration Based: photo

Color or Black and White: bw

Description / Notes: Photograph of a father inscribing a photo while Mother and young baby pose.

Binder: $1914-19162$ of 5

Year: 1915

Slogan: At the Front with the Autographic Kodak

Subject(s): product - camera

Product: Autographic Kodak

Description / Notes: Article about James H. Hare, war

correspondent who uses a Kodak Autographic Camera

Binder: $1914-19162$ of 5

Year: 1915

Slogan: La Kodak Autografica

Subject(s): product - camera

Product: Autographic Kodak

Description / Notes: In Spanish

Binder: 1914-1916 2 of 5

Year: 1915

Slogan: "Eastman"

Subject(s): product - film \& paper

Product: Eastman Film

Color or Black and White:

Binder: $1914-19162$ of 5

Year: 1915

Slogan: "Eastman"

Subject(s): product - film \& paper

Product: Eastman Motion Picture Film

Color or Black and White

Binder: $1914-19162$ of 5

Year: 1915

Slogan: Screen Work

Subject(s): product - medical

Product: Eastman Screen X-Ray film

Color or Black and White:

Binder: 1914-1916 2 of 5 
Year: 1915

Slogan: Film Efficiency

Subject(s): product - medical

Product: Eastman X-Ray Film

Color or Black and White:

Binder: $1914-19162$ of 5

Year: 1915

Slogan: Uniform in Results

Subject(s): product - medical

Product: Eastman X-Ray Film, Eastman Screen X-Ray Film.

Color or Black and White:

Binder: $1914-19162$ of 5

Slogan: Scout with a Kodak

Subject(s): boy

Text: none

Implied Audience: parents/children

Product: Kodak

Photo or Illustration Based: photo

Color or Black and White: bw

Description / Notes: Photograph of a boy on a rock with a

Kodak, looking at the camera, as if to photograph it.

Binder: $1914-19162$ of 5

Slogan: On Every hike--A Kodak

Subject(s): Boy

Text: none

Implied Audience: parents/children

Product: Kodak

Photo or Illustration Based: photo

Color or Black and White: bw

Description / Notes: Photograph of a boy with a camera and walking stick, hiking.

Binder: $1914-19162$ of 5

Slogan: Let your Kodak tell the Story of Days afield.

Subject(s): Boy

Text: There's interest in every moment of the picture making and then the added pleasure of the pictures themselves.

Implied Audience: parents/children

Product: Kodak

Photo or Illustration Based: photo

Color or Black and White: bw

Description / Notes: Photograph of a boy with a Kodak

photographing a bush.

Binder: $1914-19162$ of 5

Slogan: There are no Game Laws - and no accidents - for those

who hunt with a Kodak.

Subject(s): boy and girl

Text: none

Implied Audience: parents/children / outdoors

Product: Kodak

Photo or Illustration Based: photo

Color or Black and White: bw

Description / Notes: Photograph of a young boy with a gun, and a young girl with a camera in a field.

Binder: 1914-1916 2 of 5

Slogan: Kodak System

Subject(s): boy with camera

Text: Every out-of-doors day offers opportunity for picture taking

- and there's more than a passing pleasure in a collection of

pictures of your sports and your chums. You think more of such

pictures every day and year. Ask father what he'd give now for a

photographic story of his boyhood.

Implied Audience: parents/children

Product: Kodak

Photo or Illustration Based: photo

Color or Black and White: bw

Description / Notes: Photograph of a boy with skates and a

camera sitting outdoors.

Binder: 1914-1916 2 of 5

Slogan: Let the Children Kodak

Subject(s): children

Text: none 
Implied Audience: families

Product: Kodak

Photo or Illustration Based: photo

Color or Black and White: bw

Description / Notes: photograph of an older girl and younger

boy, girl writing on the back with Autographic Kodak

Binder: 1914-1916 2 of 5

Slogan: Let the Children Kodak

Subject(s): children

Text: The pictures they will make of each other, of their pets and

playmates, of their games and romps, will prove as great a delight

to you as to them. Such pictures will find their way into the Kodak

Album and will preserve for all the family, the story of the

childhood that they are so rapidly growing out of. Even in

Kindergarten days they can make good pictures wit the Brownies and as they approach the "teens" can readily operate any Kodak.

Every step in picture making is simple by the Kodak method--and

it's less expensive than you think.

Implied Audience: families

Product: Kodak

Photo or Illustration Based: photo

Color or Black and White: bw

Description / Notes: same photo as above, but with added text.

Binder: $1914-19162$ of 5

Slogan: The always welcome gift - A Kodak

Subject(s): father / son in parlor

Text: The gift that keeps the picture of every youthful interest -

School days and sports, the winter and summer outings, the city boy's trip to the country and the country boy's trip to the city. In all these there is fun in the picture taking and afterwards both fun and satisfaction in possession.

Implied Audience: parents/children

Product: Kodak

Photo or Illustration Based: photo

Color or Black and White: bw

Description / Notes: Photograph of a father and son in the

parlor, the father holding the camera while sitting in an armchair, the son leaning against the hearth.

Binder: 1914-1916 2 of 5

Slogan: Her Christmas Morning Kodak

Subject(s): Kodak Girl

Text: The gift that provides the means for keeping a happy picture story of the day.

Implied Audience: family / Christmas

Product: Kodak

Photo or Illustration Based: photo

Color or Black and White: bw

Description / Notes: Photograph of a woman with Kodak in her arms, dressed in a sweater, gloves, and hat.

Binder: 1914-1916 2 of 5

Slogan: On every outing--Kodak

Subject(s): Kodak Girl

Text: Let picture taking add to the delights of country life.

Everyday in the open, every picnic party is made merrier if you

Kodak--and afterwards comes in the pleasure in the pictures

themselves, and picture taking is less expensive than you think.

Implied Audience: women

Product: Kodak

Photo or Illustration Based: illus

Color or Black and White: bw

Description / Notes: Illustration of a woman on a branch, photographing nature.

Binder: $1914-19162$ of 5

Slogan: Take a Kodak with You

Subject(s): Kodak Girl

Text: Double the fun and prolong the pleasures of every outing with Kodak pictures.

Implied Audience: women

Product: Kodak

Photo or Illustration Based: illus

Color or Black and White: bw

Description / Notes: Illustration of a woman outside a car photographing nature, while another woman watches from within 
the car.

Binder: 1914-1916 2 of 5

Slogan: Holidays are Kodak Days

Subject(s): Kodak girl / couple in horse and buggy

Text: Every winter outing, every home coming of the boys and

girls, the Christmas and New Years festivities - in each of these are fascinating subjects for the Kodak - pictures that make fun in the

taking and that to you will always prove a delight. Picture taking by daylight or flashlight, is simple by the Kodak method, and it's not expensive now a days. Put Kodak on your Christmas list.

Implied Audience: outdoors / Christmas

Product: Kodak

Photo or Illustration Based: illus

Color or Black and White: bw

Description / Notes: Illustration of a woman photographing a

couple in a horse-drawn sleigh.

Binder: 1914-1916 2 of 5

Slogan: On every out-of-doors day-- Kodak

Subject(s): Kodak Girls

Text: none

Implied Audience: women

Product: Kodak

Photo or Illustration Based: photo

Color or Black and White: bw

Description / Notes: Photograph of two women on a pier, one photographing, the other with a camera on her shoulder.

Binder: $1914-19162$ of 5

Slogan: There are no Game Laws for Those Who Hunt With a Kodak

Subject(s): man and deer in the snow

Text: none

Implied Audience: outdoorsmen

Product: Kodak

Photo or Illustration Based: photo

Color or Black and White: bw

Description / Notes: Photograph of a man photographing two deer, in the snow in the forest.

Binder: $1914-19162$ of 5

Slogan: Put a Kodak in your pocket

Subject(s): man in a suit

Text: Have it ready for the unexpected that always happens.

There's a size for the vest as well as the coat. Kodak, you know, means photography with the bother left out.

Implied Audience: men / general

Product: Kodak

Photo or Illustration Based: photo

Color or Black and White: bw

Description / Notes: Photograph of a man in a suit, with camera in his pocket.

Binder: 1914-1916 2 of 5

Slogan: There are no Game Laws for Those Who Hunt With a Kodak

Subject(s): Man with a gun and two women

Text: none

Implied Audience: outdoorsmen

Product: Kodak

Photo or Illustration Based: photo

Color or Black and White: bw

Description / Notes: Photograph of a man with a gun, reading a game law sign on a tree, while two women watch.

Binder: 1914-1916 2 of 5

Slogan: Keep a Kodak Story of the Children

Subject(s): two women / two children

Text: Let your films make fast for you the impressions of those

childhood days that are so soon outgrown. There is a new delight in every moment of the story making--and a lasting pleasure in

the pictures themselves.

Implied Audience: families

Product: Kodak

Photo or Illustration Based: photo

Color or Black and White: bw

Description / Notes: Photo of two women on a beach, one 
photographing two young children holding hands.

Binder: 1914-1916 2 of 5

Slogan: Let the Children Kodak

Subject(s): two young boys

Text: Add to the collection of your pictures of the children and to the more formal studio pictures that you will surely want of them, the care free, unstudied pictures that they will make of each other - pictures that are free from constraint, filled with the naturalness of childhood.

Implied Audience: families

Product: Kodak

Photo or Illustration Based: photo

Color or Black and White: bw

Description / Notes: Photograph of two young boys on a beach, taking photos.

Binder: 1914-1916 2 of 5

Slogan: Let the Children Kodak

Subject(s): young girl and boy

Text: none

Implied Audience: families

Product: Kodak

Photo or Illustration Based: photo

Color or Black and White: bw

Description / Notes: Photo of a young girl and boy on a porch, the young girl poses for the boy.

Binder: $1914-19162$ of 5

Slogan: Kodak Dominates

Subject(s): product - misc

Text: Not alone because it is first in photographic invention and progress, but because it so encourages and rewards the taking of infinite pains that honest workmanship has become a habit in the Kodak factories.

Product: Kodak branding

Photo or Illustration Based: photo

Color or Black and White: bw

Description / Notes: photograph of a man working on a lens for a Kodak

Binder: $1914-19162$ of 5

Year: 1915

Slogan: Kodak

Subject(s): product - camera

Product: Kodak Cameras

Description / Notes: In Spanish

Binder: 1914-1916 2 of 5

Year: 1915

Slogan: Holidays are Kodak Days

Subject(s): product - camera

Product: Kodak Cameras

Color or Black and White:

Binder: 1914-1916 2 of 5

Year: 1915

Slogan: Kodak

Subject(s): product - camera

Product: Kodak Cameras

Description / Notes: In Spanish

Binder: $1914-19162$ of 5

Year: 1915

Slogan: Kodaks

Subject(s): product - camera

Product: Kodak Cameras

Description / Notes: In Spanish

Binder: 1914-1916 2 of 5

Slogan: All Out-doors invites your Kodak

Subject(s): two men

Text: No trip too long, no conditions too rough for a Kodak outfit.

You can take, title and finish the negatives on the spot by the

Kodak system. You can make sure.

Implied Audience: outdoorsmen

Product: Kodak system

Photo or Illustration Based: photo 


\section{Color or Black and White: bw}

Description / Notes: Photograph of two men in the snow,

looking at a roll of developed snow.

Binder: $1914-19162$ of 5

Year: 1915

Slogan: The New Folding Autographic Brownie

Subject(s): product - camera

Product: No. 2 Folding Autographic Brownie

Description / Notes: Price: $\$ 6.00$. With rapid rectilinear lens: $\$ 7.50$

Binder: $1914-19162$ of 5

Year: 1915

Slogan: There's a photographer in your town

Subject(s): none

Text: Your friends can buy anything you can send (crossed out)

give them - except your photograph.

Product: photograph

Photo or Illustration Based: Text: Color or Black and White:

bw

Description / Notes: no photo- text only. One word is crossed

out - looks like that is part of the ad.

Binder: 1914-1916 2 of 5

Year: Mar-16

Slogan: There's a photographer in your town

Subject(s): none

Text: Let the Easter card carry the message that lends the

personal touch of friendship: Your photograph.

Product: photograph

Photo or Illustration Based: photo

Color or Black and White: bw

Description / Notes: emphasis on text - but there is transparent lily photograph in the back.

Binder: $1914-19162$ of 5

Year: Oct. 1915

Slogan: There's a photographer in your town
Subject(s): none

Text: Photographs, like the family furniture are most cherished when very new or very old. Mother banished her mother's old sofa to the attic. You, the grand-daughter, brought it down again and you prize it, even above the latest "new piece" It's the same with pictures- your photograph just as you now are- your friends will appreciate, now. And the second generation will cherish it even more.

Implied Audience: families

Product: photographs

Photo or Illustration Based: Text: Color or Black and White: bw

Description / Notes: no photo- text only.

Binder: $1914-19162$ of 5

Slogan: There's a photographer in your town

Subject(s): none

Text: That earliest slight break in home ties - the morning when the boy or the girl first trudges off to school! From that day, the changes are rapid. Every year you note them. And, almost before you realize it, there comes the severer sundering of those ties, when John or Mary with a cheery "Will be home for Christmas, sure," waves a stout farewell. Both of you are choking back sentiment. And afterward-how pictures, showing all the rapid transitions, do help.

Implied Audience: families

Product: photographs

Photo or Illustration Based: Text: Color or Black and White: bw

Description / Notes: no photo- text only.

Binder: $1914-19162$ of 5

Year: Nov-15

Slogan: There's a photographer in your town

Subject(s): none

Text: For Christmas- your photograph. The simple gift that lends the touch of friendship without the embarrassment of an obligation Product: photographs

Photo or Illustration Based: Text: Color or Black and White: 
bw

Description / Notes: no photo- text only.

Binder: 1914-1916 2 of 5

Year: 1915

Slogan: Results

Subject(s): product - medical

Product: Seed X-Ray Plates

Color or Black and White:

Binder: 1914-1916 2 of 5

Year: 1915

Slogan: An Ideal Plate

Subject(s): product - medical

Product: Seed X-Ray Plates

Color or Black and White:

Binder: $1914-19162$ of 5

Year: 1915

Slogan: X-Ray Examinations

Subject(s): product - medical

Product: Seed X-Ray Plates

Color or Black and White:

Binder: $1914-19163$ of 5

Year: 1916

Slogan: A New Kodak in a New Size

Subject(s): product - camera

Product: 2c Kodak Autographic Jr.

Description / Notes: Price: $\$ 12.00, \$ 14.00$, and $\$ 19.00$

Binder: 1914-1916 3 of 5

Year: 1916

Slogan: The New 2c Kodak Jr.

Subject(s): product - camera

Product: 2c Kodak Jr.

Description / Notes: Price: $\$ 12.00$ to $\$ 19.00$

Binder: $1914-19163$ of 5
Year: 1916

Slogan: Eastman Dental X-Ray Film

Subject(s): product - medical

Product: Eastman Dental X-Ray Films

Description / Notes: X-Rays of teeth caption: "Courtesy of Dr.

Albert Kinley, Boston, Mass."

Binder: $1914-19163$ of 5

Year: 1916

Slogan: Prove your Case!

Subject(s): product - medical

Product: Eastman Dental X-Ray Films

Description / Notes: X-Ray caption: "No. 1 Dental X-Ray Film Radiographs"

Binder: $1914-19163$ of 5

Year: 1916

Slogan: If there's any doubt, use Eastman Dental X-Ray Film

Subject(s): product - medical

Product: Eastman Dental X-Ray Films

Description / Notes: X-Rays of teeth caption: "Courtesy of Dr. Albert Kinley, Boston, Mass."

Binder: $1914-19163$ of 5

Year: 1916

Slogan: The reason why is shown by Eastman Dental X-Ray Film

Subject(s): product - medical

Product: Eastman Dental X-Ray Films

Description / Notes: X-Rays of teeth caption: "Courtesy of Dr. Albert Kinley, Boston, Mass."

Binder: 1914-1916 3 of 5

Year: 1916

Slogan: You can stand by the verdict of Eastman Dental X-Ray Film

Subject(s): product - medical

Product: Eastman Dental X-Ray Films

Description / Notes: X-Rays of teeth caption: "Courtesy of Dr. Albert Kinley, Boston, Mass." 
Binder: $1914-19163$ of 5

Year: 1916

Slogan: All Kodak Film is now Speed Film

Subject(s): product - film \& paper

Product: Eastman N.C. Film, and Speed Film

Color or Black and White:

Binder: 1914-1916 3 of 5

Year: 1916

Slogan: Introducing the Improved--Eastman X-Ray Film

Subject(s): product - medical

Product: Eastman X-Ray Film

Color or Black and White:

Binder: 1914-1916 3 of 5

Year: 1916

Slogan: Restults with the improved--Eastman X-Ray Film

Subject(s): product - medical

Product: Eastman X-Ray Film

Description / Notes: X-Ray caption: "Courtesy of Dr. J.T. Case, Roentgenologist, The Battle Creek Sanitarium"

Binder: 1914-1916 3 of 5

Year: 1916

Slogan: Prove the diagnosis with Eastman X-Ray Film

Subject(s): product - medical

Product: Eastman X-Ray Film

Description / Notes: X-Ray caption: "Courtesy of Dr. J.T. Case, Roentgenologist, The Battle Creek Sanitarium"

Binder: $1914-19163$ of 5

Year: 1916

Slogan: The proof in diagnosis

Subject(s): product - medical

Product: Eastman X-Ray Film

Color or Black and White:

Binder: $1914-19163$ of 5
Year: 1916

Slogan: Make the Diagnosis Easier, use Eastman X-Ray Film

Subject(s): product - medical

Product: Eastman X-Ray Film

Color or Black and White:

Binder: $1914-19163$ of 5

Year: 1916

Slogan: Eastman X-Ray Film

Subject(s): product - medical

Product: Eastman X-Ray Film

Color or Black and White:

Binder: $1914-19163$ of 5

Slogan: A Happy Christmas Thought --Kodak

Subject(s): Kodak Girl

Text: The gift that adds to the good times at the moment; that indoors and out gives zest to the merry making and then

preserves the happy picture story of all that goes to make the day a merry one.

Implied Audience: women / Christmas

Product: Kodak

Photo or Illustration Based: photo

Color or Black and White: bw

Description / Notes: Photograph of a woman in an armchair,

holding a camera. Presents behind her.

Binder: $1914-19163$ of 5

Slogan: A Happy Christmas Thought --Kodak

Subject(s): Kodak Girl

Text: The gift that adds to the good times at the moment; that

indoors and out gives zest to the merry making and then

preserves the happy picture story of all that goes to make the day a merry one.

Implied Audience: women / Christmas

Product: Kodak

Photo or Illustration Based: illus

Color or Black and White: bw

Description / Notes: an illustration of the above photograph - 
same text.

Binder: $1914-19163$ of 5

Slogan: A Happy Christmas Thought --Kodak

Subject(s): Kodak Girl

Text: The gift that adds to the good times at the moment; that

indoors and out gives zest to the merry making and then

preserves the happy picture story of all that goes to make the day a merry one.

Implied Audience: women / Christmas

Product: Kodak

Photo or Illustration Based: photo

Color or Black and White: bw

Description / Notes: Same text as above, but with different

photo - a woman in a hat, with a camera.

Binder: $1914-19163$ of 5

Year: 1916

Slogan: Kodak as you Go

Subject(s): Kodak girls

Text: The delights of old companionships, the charm of new and interesting friends, the thrill of novelty that comes with new

scenes and a change of routine-- All these mean more to those

who Kodak.. There's a joyful fascination in the picture taking. And afterward the Kodak Album brings the added delight of living over again the happy days of that "too short vacation"

Implied Audience: women / travelers

Product: Kodak

Photo or Illustration Based: photo

Color or Black and White: bw

Description / Notes: Photograph of two women in a (very nice)

train car, loading film, and possibly reading the manual.

Binder: $1914-19163$ of 5

Year: 1916

Slogan: Kodak as you Go

Subject(s): Kodak girls

Text: Kodak photography is so simple that you can make good

pictures from the very start--and is less expensive than you think.
Implied Audience: women / travelers

Product: Kodak

Photo or Illustration Based: photo

Color or Black and White: bw

Description / Notes: same photo as above, but with different

text.

Binder: 1914-1916 3 of 5

Slogan: Take a Kodak with You

Subject(s): man, woman

Text: none

Implied Audience: trave

Product: Kodak

Photo or Illustration Based: photo

Color or Black and White: bw

Publications: Independent, Sunset, and American, Colliers,

Travel, Scientific American

Description / Notes: Photograph of a young couple outside their car, the woman with the camera, photographing a blacksmith [?] with a pipe. The woman is wearing a mink.

Binder: $1914-19163$ of 5

Year: 1916

Slogan: There's a photographer in your town

Subject(s): none

Text: Your friends can buy anything you can give them - except

your photograph

Product: Kodak

Photo or Illustration Based: Text: Color or Black and White: bw

Description / Notes: No photo - text only. Similar to the other version, but without the crossed out word

Binder: $1914-19163$ of 5

Year: 1916

Slogan: "Kodak"

Subject(s): product - misc.

Product: Kodak Brand

Description / Notes: "Is our registered and common-law trade- 
mark and cannot be rightfully applied except to goods of our manufacture"

Binder: $1914-19163$ of 5

Year: 1916

Slogan: There's winter fun too--in a Kodak

Subject(s): product - camera

Product: Kodak camera

Color or Black and White:

Binder: $1914-19163$ of 5

Year: 1916

Slogan: All Kodak Film Now Speed Film

Subject(s): product - film \& paper

Product: Kodak Film

Color or Black and White:

Binder: $1914-19163$ of 5

Year: 1916

Slogan: The Thing Worth While

Subject(s): product - misc.

Product: Kodak Workmanship

Color or Black and White:

Binder: $1914-19163$ of 5

Year: 1916

Slogan: An Institution

Subject(s): product - misc.

Product: Kodak Workmanship

Color or Black and White:

Binder: $1914-19163$ of 5

Year: 1916

Slogan: No. 2C Autographic Kodak Jr.

Subject(s): product - camera

Product: No. 2c Autographic Kodak Jr.

Description / Notes: Price: $\$ 12.00$

Binder: 1914-1916 3 of 5
Year: 1916

Slogan: A Camera that fits in the pocket. A picture that fits the

view. No. 2c Folding Autographic Brownie

Subject(s): product - camera

Product: No. 2c Folding Autographic Brownie

Description / Notes: Price: $\$ 9.00$ and $\$ 11.00$

Binder: $1914-19163$ of 5

Year: 1916

Slogan: There's a photographer in your town

Subject(s): none

Text: Christmas 1916. Your friends can buy anything you can give them--except your photograph.

Implied Audience: Christmas

Product: photograph

Photo or Illustration Based: Text: Color or Black and White: bw

Description / Notes: no photo- text only.

Binder: $1914-19163$ of 5

Year: 1917?

Slogan: There's a photographer in your town

Subject(s): none

Text: At Easter-tide. Your friends can buy anything you can give

them--except your photograph.

Implied Audience: Easter / general

Product: photograph

Photo or Illustration Based: Text: Color or Black and White: bw

Description / Notes: no photo- text only.

Binder: $1914-19164$ of 5

Year: 1916

Slogan: The New 2c Brownie

Subject(s): product - camera

Product: 2c Brownie

Description / Notes: Price: $\$ 11.00$

Binder: 1914-1916 4 of 5 
Year: 1917

Slogan: Put a "two-c" in your pocket. It fits.

Subject(s): product - camera

Product: 2c Kodak Jr.

Description / Notes: Price: $\$ 12.00$ - \$19.00

Binder: $1914-19164$ of 5

Year: 1917

Slogan: The thin, slim camera that fits in the pocket

Subject(s): product - camera

Product: 2c Kodak Jr.

Description / Notes: Price: $\$ 12.00$ to $\$ 19.00$

Binder: 1914-1916 4 of 5

Slogan: Autographic Kodak

Subject(s): cow

Text: Every sale of live-stock, especially by mail, is made more

easily when the description is backed by pictures. A close up of the head, to show character and virility, (with the body left out to avoid distortion) and then a picture taken from each side with the animal parallel to the camera to show markings and conformation -- such pictures are simple to make and each one can bear the date, and name of the animal on the negative if you use an Autographic Kodak.

Implied Audience: farmers

Product: autographic Kodak

Photo or Illustration Based: photo

Color or Black and White: bw

Description / Notes: photograph of cows. Caption reads "From a Kodak Negative"

Binder: $1914-19164$ of 5

Year: 1917

Slogan: The High record, trap-nested hen, the A.R.O. Cow, the well finished steer, the well grown colt, the prize hog:

Subject(s): farmer, chicken

Text: Whatever you raise that's a bit better than the ordinary, is worth an auto-photo-graphic record. And you can make such a record, with the data on the negative. It's a simple, permanent, inexpensive and almost instantaneous process with an Autographic Kodak.

Implied Audience: farmers

Product: autographic Kodak

Photo or Illustration Based: photo

Color or Black and White: bw

Description / Notes: photograph of a man with a chicken, inscribing something on his camera.

Binder: 1914-1916 4 of 5

Slogan: Keep an Autographic record of the children.

Subject(s): mother / child with dog.

Text: Pictures of the children, just as they are, romping about the yard with their pets or at play in the nursery. And on every

negative, written at the time, the all important date and title. It's a very simple and almost instantaneous process with an Autographic Kodak

Implied Audience: families

Product: autographic Kodak

Photo or Illustration Based: photo

Color or Black and White: bw

Description / Notes: Photograph of a mother inscribing on the

camera, while daughter and dog pose.

Binder: $1914-19164$ of 5

Year: 1917

Slogan: Keep a Kodak Story of the Baby

Subject(s): mother, father, and baby

Text: And, along with it, written on the film at the time, keep the dates and titles. How old was Baby when this was taken? Where were we the year that that was taken? Suck records mean a great deal when baby has begun outgrowing baby ways and time has begun playing tricks with memory. And to make an authentic, permanent record, on the negative it a simple and almost instantaneous process with an Autographic Kodak.

Implied Audience: families

Product: autographic Kodak

Photo or Illustration Based: photo

Color or Black and White: bw 
Description / Notes: Photograph of young parents, mother holding the baby, father inscribing something on the film.

Binder: $1914-19164$ of 5

Slogan: Spring-time on the Farm.

Subject(s): Pigs

Text: Every season is a season of interest there things are growing. And whatever is of interest is worth a picture. The pigs and the calves, the colts, the poultry and the growing crops, always offer pictures that are worth while--that are full of the intensest interest and that often become of value in selling and in record keeping. Pictures of crops, or ditching, of the orchard, all help to make an invaluable record on every farm that is systematically operated. Pictures of the tumbled down line fence to sent the absentee landlord who owns the farm next door, pictures of the bad spot in the road and the broken culvert to send the town board are often worth the whole cost of a photographic outfit. You can make such pictures easily and economically and can completely record with a date and title on the negative with an Autographic Kodak

Implied Audience: farmers

Product: autographic Kodak

Photo or Illustration Based: photo

Color or Black and White: bw

Description / Notes: Photograph of pigs. Caption reads "from a

Kodak negative."

Binder: $1914-19164$ of 5

Year: 1917

Slogan: Those things that need attention on the farm

Subject(s): product - camera

Product: Autographic Kodak

Color or Black and White:

Binder: $1914-19164$ of 5

Year: 1917

Slogan: Write it on the film - at the time.

Subject(s): Soldiers

Text: Make every negative more valuable by permanently recording at the time of exposure the all important date and title. It's a simple and almost instantaneous process with an Autographic Kodak

Implied Audience: soldiers

Product: autographic Kodak

Photo or Illustration Based: photo

Color or Black and White: bw

Description / Notes: First appearance of soldiers! Photograph of three soldiers, one holding a camera, and inscribing.

Binder: $1914-19164$ of 5

Year: 1917

Slogan: The Brownie Boy

Subject(s): Boy

Text: There's great fun out-doors in taking Brownie pictures and there's great fun in-doors in the developing and printing. There's not only the fun of having the pictures but the little camera and the literature we furnish with it, (including the magazine Kodakery, free for a whole year) gives a good foundation in photography.

Implied Audience: parents/children

Product: brownie

Photo or Illustration Based: photo

Color or Black and White: bw

Description / Notes: Photograph of a boy reading Kodakery, carrying a Brownie, outdoors.

Binder: $1914-19164$ of 5

Slogan: Hike with a Brownie

Subject(s): boy scouts

Text: Whether you go for a month in a camp or for a Saturday hike, take along a camera. There are worth while pictures every time--pictures of the things you see, and above all, pictures of the fellows. And you can make them. It's all very simple with a Brownie or a Kodak, even to the developing and printing. No darkroom is necessary and it's all easier and less expensive than you think.

Implied Audience: parents/children

Product: brownie

Photo or Illustration Based: photo 


\section{Color or Black and White: bw}

Description / Notes: Photograph of five boys crowded around a

Brownie, taking photos, aiming at the camera.

Binder: $1914-19164$ of 5

Slogan: A Brownie for her Birthday

Subject(s): mother / young girl

Text: Even in their kindergarten days, almost from the time they

can count the candles on their birthday cake, the children make

really good pictures with their simple little Brownie cameras. It's

good fun for them, and the pictures they make of each other are

among the treasured ones that find their way into the family snapshot album.

Implied Audience: families

Product: Brownie

Photo or Illustration Based: photo

Color or Black and White: bw

Description / Notes: Photograph of a mother and daughter, the daughter with a Brownie. Birthday cake in the BG.

Binder: $1914-19164$ of 5

Year: 1916

Slogan: The Court of Final Judgment

Subject(s): product - medical

Product: Eastman Dental X-Ray Films

Description / Notes: X-Rays of teeth caption: "Courtesy of Dr. Albert Kinley, Boston, Mass."

\section{Binder: $1914-19164$ of 5}

Year: 1917

Slogan: Make it conclusive--use Eastman X-Ray Dental Films

Subject(s): product - medical

Product: Eastman Dental X-Ray Films

Color or Black and White:

Binder: $1914-19164$ of 5

Year: 1917

Slogan: Practically Indispensable

Subject(s): product - medica
Product: Eastman Dental X-Ray Films

Color or Black and White:

Binder: $1914-19164$ of 5

Year: 1917

Slogan: Put your "O.K." On your work

Subject(s): product - medical

Product: Eastman Dental X-Ray Films

Color or Black and White:

Binder: $1914-19164$ of 5

Year: 1917

Slogan: Employ a diagnostician

Subject(s): product - medical

Product: Eastman Dental X-Ray Films

Description / Notes: X-Ray caption: "Courtesy of Dr. S.M.

Getzoff"

Binder: $1914-19164$ of 5

Year: 1916

Slogan: Make your Diagnosis certain, by using Eastman X-Ray

Film

Subject(s): product - medical

Product: Eastman X-Ray Film

Color or Black and White:

Binder: $1914-19164$ of 5

Year: 1917

Slogan: Certify your Diagnoses--use Eastman Dental X-Ray film

Subject(s): product - medical

Product: Eastman X-Ray Film

Color or Black and White:

Binder: 1914-1916 4 of 5

Slogan: Every out-of-doors sport invites your Kodak

Subject(s): boy

Text: none

Implied Audience: parents/children

Product: Kodak 
Photo or Illustration Based: photo

Color or Black and White: bw

Description / Notes: Photograph of a boy in a baseball uniform holding a Kodak, bat.

Binder: $1914-19164$ of 5

Year: 1917

Slogan: It's a Kodak!

Subject(s): boy scouts

Text: none

Implied Audience: parents/children

Product: Kodak

Photo or Illustration Based: photo

Color or Black and White: bw

Description / Notes: Photograph of a boy with a Kodak in the air, yelling, presumably to his friends. Outdoors.

Binder: $1914-19164$ of 5

Slogan: The Kodak in Camp

Subject(s): boy scouts

Text: Every kind of out-door fun becomes more fun with a Kodak along.

Implied Audience: parents/children

Product: Kodak

Photo or Illustration Based: photo

Color or Black and White: bw

Description / Notes: Photograph of two boy scouts, one posing with a tent, the other photographing him.

Binder: $1914-19164$ of 5

Slogan: Kodak on the Farm

Subject(s): Chicken

Text: Every corner on the farm has its point of interest, its picture worth taking. From the dairy to the poultry house and down in the orchard it is filled with picture opportunities. And in the farm house, as in every other home, Kodak finds its happiest subjects among the children and their friends.

Implied Audience: Farmers

Product: Kodak
Photo or Illustration Based: photo

Color or Black and White: bw

Description / Notes: Photograph of a chicken. Caption reads : From a Kodak negative.

Binder: 1914-1916 4 of 5

Slogan: The High record, trap-nested hen, the A.R.O. Cow, the well finished steer, the well grown colt, the prize hog:

Subject(s): farmer, chicken

Text: Whatever you raise that's a bit better than the ordinary, is worth an auto-photo-graphic record. And you can make such a record, with the data on the negative. It's a simple, permanent, inexpensive and almost instantaneous process with an Autographic Kodak.

Implied Audience: farmers

Product: Kodak

Photo or Illustration Based: illustration

Color or Black and White: bw

Description / Notes: Illustration of same ad with a photograph.

Binder: $1914-19164$ of 5

Slogan: Kodak, as you go

Subject(s): Kodak Girl

Text: Wherever the purr of your motor lures you, wherever the call of the road leads you, there you will find pictures, untaken pictures that invite your Kodak--intimate pictures of people and places that you and your friends can enjoy again and again as you thumb the leaves of your Kodak album. And you can take them.

Implied Audience: women

Product: Kodak

Photo or Illustration Based: photo

Color or Black and White: bw

Description / Notes: photograph of a woman in a convertible, in the drivers seat, leaning her camera out the window, taking a photograph.

Binder: $1914-19164$ of 5

Year: 1917

Slogan: Kodak, as you go 
Subject(s): Maid, woman in car

Text: Tell the tale of the long road with Kodak pictures of the interesting places you visit and the even more interesting people you meet. You and your friends will enjoy such pictures again and again as you pore over the pages of your Kodak album. And you can take them.

Implied Audience: women / general

Product: Kodak

Photo or Illustration Based: photo

Color or Black and White: bw

Description / Notes: Photo of a maid handing the camera off to

a woman in the car, the woman's hand only is visible.

Binder: $1914-19164$ of 5

Slogan: Kodak, as you go

Subject(s): Maid, woman in car

Text: none

Implied Audience: women / general

Product: Kodak

Photo or Illustration Based: photo

Color or Black and White: bw

Description / Notes: Same as other ad with the maid, but with no Text:

Binder: 1914-1916 4 of 5

Slogan: There are no Game Laws for Those Who Hunt With a

Kodak

Subject(s): Two hunters in a canoe, deer

Text: none

Implied Audience: hunters / outdoorsman

Product: Kodak

Photo or Illustration Based: photo

Color or Black and White: bw

Description / Notes: Photograph of two men in a canoe,

photographing a deer with a flash. Night photograph.

Binder: $1914-19164$ of 5

Slogan: Keep a Kodak Story of the Children

Subject(s): Two women, baby

Text: From baby days, up through the kindergarten, through school, and high-school days, it grows in interest with every chapter.

Implied Audience: families

Product: Kodak

Photo or Illustration Based: photo

Color or Black and White: bw

Description / Notes: Photograph of a woman with a camera photographing another woman with a baby at a waterfront.

Binder: $1914-19164$ of 5

Slogan: Let the Children Kodak

Subject(s): boy with dog

Text: Next to the possession of pets themselves the youngsters enjoy making pictures of the dogs and cats and ponies of which they make companions. And invariably they will make pictures of each other, pictures that have the charm of genuine naturalness, so delightfully "as they are" that you will demand prints for your own album. And you will be surprised at what good pictures the youngsters can take with a simple little Kodak or Brownie.

Implied Audience: families

Product: Kodak / brownie

Photo or Illustration Based: photo

Color or Black and White: bw

Description / Notes: Photograph of a boy and his dog, the boy posing the dog on the steps.

Binder: 1914-1916 4 of 5

Slogan: At home with the Kodak

Subject(s): mother/children

Text: Wherever there are children there is work for your Kodak, work that is even more fascinating than the picture records of your travels. And such pictures live, live because they hold the touch of human interest--because they keep for to-morrow, then they are grown up, the story of the children as they are to-day. You can make such pictures; the children can make them--with Kodak or Brownie.

Implied Audience: families

Product: Kodak / brownie

Photo or Illustration Based: photo 


\section{Color or Black and White: bw}

Description / Notes: photograph of a mother with camera on a

tripod photographing two children.

Binder: $1914-19164$ of 5

Slogan: With Kodak and Brownie

Subject(s): two children

Text: You can get more and more fun from your in-door and outdoor sports. Pictures of your friends and your pets and the places you visit make fun in the taking and you will always enjoy the pictures as you look over the Kodak album.

Implied Audience: parents/children

Product: Kodak / brownie

Photo or Illustration Based: photo

Color or Black and White: bw

Description / Notes: photograph of a girl inscribing onto an

Autographic Kodak while her brother watches, with a Brownie at his side.

Binder: 1914-1916 4 of 5

Year: 1917

Slogan: Kodak knows no dark days

Subject(s): man, woman

Text: With its allies, the Kodak flash sheets and a Kodak flash sheet holder, youth Kodak camera is ready for every picture opportunity.

Implied Audience: women / general

Product: Kodak flashes

Photo or Illustration Based: photo

Color or Black and White: bw

Description / Notes: Photograph of a young couple, the woman posing in the corner, the man holding the flash, camera on tripod. Both are dressed in fancy clothes.

Binder: $1914-19164$ of 5

Slogan: The Witchery of Kodakery

Subject(s): three children

Text: Right at home there are hundreds of pictures worth the

taking--pictures of children and pets, pictures of all that makes the home home. And even the children can do the developing and printing--also, right at home.

Implied Audience: families

Product: Kodak system

Photo or Illustration Based: photo

Color or Black and White: bw

Description / Notes: Photo of three children, at home developing film.

Binder: $1914-19164$ of 5

Year: 1916

Slogan: The New Model 3a Brownie

Subject(s): product - camera

Product: Model 3a Brownie

Description / Notes: Price: $\$ 10.00, \$ 12.00$

Binder: $1914-19164$ of 5

Year: 1916

Slogan: There's winter sport too--in a Kodak

Subject(s): Kodak Girl

Text: Outdoors with skates and skis and coasting, when the trees are snow laden and great drifts tell the tale of the last nights

storm, there are pictures well worth taking. And indoors there are group pictures and home portraits, by daylight or flashlight and in the long winter evenings, when rain and sleet make the outdoors impossible, there's good fun in developing and printing. Every step is simple by the Kodak system.

Implied Audience: women / general

Product: photograph

Photo or Illustration Based: photo

Color or Black and White: bw

Publications: Cosmopolitan

Description / Notes: photograph of a woman with a Kodak, in a winter coat, hat, and scarf.

Binder: $1914-19164$ of 5

Year: 1917

Slogan: Seed X-Ray Plates

Subject(s): product - medical 
Product: Seed X-Ray Plates

Description / Notes: X-Ray caption: "From a seed X-ray plate"

Binder: $1914-19164$ of 5

Year: 1917

Slogan: Vest Pocket Autographic Kodak, the vest pocket camera

that will really go in the vest pocket

Subject(s): product - camera

Product: Vest Pocket Autographic Kodak

Description / Notes: Price: $\$ 6.00$ to $\$ 22.00$

Binder: $1914-19164$ of 5

Year: 1917

Slogan: Your other camera

Subject(s): product - camera

Product: Vest Pocket Kodak

Description / Notes: Price: $\$ 6.00$ to $\$ 22.50$

Binder: $1914-19165$ of 5

Year: 1917

Slogan: The 2c Kodak, Junior

Subject(s): product - camera

Product: 2c Kodak Jr.

Description / Notes: Price: $\$ 12.00$ to $\$ 19.00$

Binder: $1914-19165$ of 5

Year: 1917

Slogan: Build your reputation on facts

Subject(s): product - medical

Product: Eastman Dental X-Ray Films

Description / Notes: X-Ray Caption: "Courtesy of Federal X-Ray Co"

Binder: $1914-19165$ of 5

Year: 1917

Slogan: Get Advance Information

Subject(s): product - medical

Product: Eastman Dental X-Ray Films

Description / Notes: X-Ray caption: "Courtesy of Dr. S.M.

\section{Getzoff"}

Binder: $1914-19165$ of 5

Year: 1917

Slogan: You Owe it to your patients to make a preliminary

examination with Eastman Dental X-Ray Film

Subject(s): product - medical

Product: Eastman Dental X-Ray Films

Description / Notes: X-Ray caption: "Courtesy of Dr. S.M. Getzoff"

Binder: $1914-19165$ of 5

Year: 1917

Slogan: "Eastman" and "Kodak"

Subject(s): product - film \& paper

Product: Eastman Motion Picture Film

Color or Black and White:

Binder: 1914-1916 5 of 5

Year: 1917

Slogan: Keep the Portraits of your Heroes

Subject(s): product - misc.

Product: Eastman Portrait Albums

Color or Black and White:

Binder: $1914-19165$ of 5

Year: 1917

Slogan: For your Convenience, Doctor--The Eastman X-Ray

Reducing Camera

Subject(s): product - medical

Product: Eastman X-Ray Reducing Camera

Description / Notes: Price: $\$ 100.00$

Binder: 1914-1916 5 of 5

Slogan: There's a photographer in your town

Subject(s): none

Text: For your Soldier Boy in camp or at the front; for your Sailor

Lad patrolling the high seas; from you to him, to make his heart

light and to help keep tight the home ties--your photograph. 
Implied Audience: soldiers / families

Product: Kodak

Photo or Illustration Based: Text: Color or Black and White: bw

Publications: The American Magazine

Description / Notes: no photo- text only.

Binder: 1914-1916 5 of 5

Slogan: Take a Kodak with You

Subject(s): Soldiers

Text: none

Implied Audience: Soldiers

Product: Kodak

Photo or Illustration Based: photograph

Color or Black and White: bw

Description / Notes: Photograph of a group of soldiers at night watching one develop film.

Binder: $1914-19165$ of 5

Year: 1917

Slogan: Kodak in Camp

Subject(s): soldiers

Text: From reveille to taps, each hour will bring something new into the life of every young soldier. New surroundings, new habits, new faces, and new friendships will make for him a new world--a world full of interest to him to-day and a world upon which he will often dwell in memory when peace has come again. And this new world of his offers Kodak opportunities that will relieve the tedium of camp routine at the time and will afterward provide what will be to him and his friends the most interesting of all books--his Kodak album. The parting gift, a Kodak. Wherever he goes the world over, he will find Kodak film to fit his Kodak.

Implied Audience: soldiers / families

Product: Kodak

Photo or Illustration Based: illus

Color or Black and White: bw

Description / Notes: Illustration of two soldiers in a tent looking at an album.
Binder: $1914-19165$ of 5

Year: 1917

Slogan: Keep your Kodak Busy

Subject(s): Soldiers

Text: "The Army lives on letters" is the way the boys at the front put it. And when those longed-for envelopes with the home town postmark contain pictures of the home folks and home doings, they go far toward making lighter hearts and happier faces. Keep your Kodak busy for the sake of the lads in the trenches, the boys in camp and on shipboard. Help keep tight the bonds between the home and those that are fighting for that home.

Implied Audience: soldiers / families

Product: Kodak

Photo or Illustration Based: photograph

Color or Black and White: bw

Publications: The American Magazine

Description / Notes: Photograph of a group of soldiers looking at photographs.

Binder: 1914-1916 5 of 5

Year: 1917

Slogan: Pictures from Home

Subject(s): soldiers

Text: Let the times temper your giving that the spirit of Christmas may be carried to out soldiers over seas. Make your gift to those at home, a Kodak, that they in turn may make light hearts and happy faces by sending a continued Kodak story of that home to the beaver lads, somewhere in France. Helpful organizations are doing a great work in looking after their physical comforts-- but 'the folks at home' are the ones who can help them keep cheerful in mind and heart-- and pictures will help.

Implied Audience: soldiers / families

Product: Kodak

Photo or Illustration Based: photo

Color or Black and White: bw

Description / Notes: Photograph of a group of soldiers looking at photographs. Caption : "Why mess was late"

Binder: 1914-1916 5 of 5 
Slogan: Snap-shots from Home.

Subject(s): Soldiers

Text: Give cheer to the boys in camp and on shipboard by sending

them pictures from home. There are likely to be some tedious, homesick days and a little cheer-up in the way of photographs of the home folks and the home doings will do them a lot of good. And some day when you want to give something a little more substantial, send along a vest pocket Kodak and ask your soldier or sailor boy to send pictures to you.

Implied Audience: soldiers / families

Product: Kodak

Photo or Illustration Based: illus

Color or Black and White: bw

Description / Notes: simpler illustration of the same add used earlier.

Binder: 1914-1916 5 of 5

Slogan: Pictures from Home

Subject(s): Soldiers

Text: Over there, with thousands of miles of sea and land between

them and home, are Our Boys, smiling and fighting--fighting with bullets, against a dogged foe; with smiles, fighting homesickness and dread monotony. It's a part of the nation's job to-day to keep those boys cheerful, to hold fast the bonds between camp and home, to make light hearts and smiling faces--and these things pictures can help to do--pictures of the home folks and the home doings, pictures of the neighbors, pictures that will enliven their memories of the days before the war--simple Kodak pictures, such as you can make. These can help

Implied Audience: soldiers / families

Product: Kodak

Photo or Illustration Based: illus

Color or Black and White: bw

Publications: The American Magazine

Description / Notes: Illustration of soldiers in a camp at night, looking at photographs.

Binder: 1914-1916 5 of 5

Year: 1917
Slogan: Take a Kodak With You

Subject(s): product - camera

Product: Kodak cameras and film

Color or Black and White:

Binder: $1914-19165$ of 5

Year: 1917

Slogan: No. 3a Autographic Kodak

Subject(s): product - camera

Product: No. 3a Autographic Kodak

Description / Notes: Price: $\$ 20.00$

Binder: $1914-19165$ of 5

Year: Jun-17

Slogan: There's a photographer in your town

Subject(s): soldier

Text: Loyalty--first to country, then to home. To cheer those who

cannot serve as you serve but whose hearts are with you--your photograph.

Implied Audience: soldiers / families

Product: photograph

Photo or Illustration Based: illus

Color or Black and White: bw

Description / Notes: illustration of a soldier.

Binder: $1914-19165$ of 5

Year: 1917

Slogan: none

Subject(s): product - medical

Product: Seed X-Ray Plates

Description / Notes: X-Ray caption: "Seed stereoscopic chest plate"

Binder: 1914-1916 5 of 5

Year: 1917

Slogan: Seed X-Ray Plates

Subject(s): product - medical

Product: Seed X-Ray Plates

Description / Notes: X-Ray caption: "Seed stereoscopic plate" 
Binder: $1914-19165$ of 5

Slogan: The Kodak Story of the War

Subject(s): Navy soldiers on a ship

Text: War isn't all fighting--but to thousands of young Americans on shipboard and in camp it will be a new world, will open up a new life. New ties will be formed, new friendships cemented. The daily routine on shipboard and in camp, the shore leave in strange cities, the pictures of comrades-- all these things will make a heart gripping Kodak story for the folks at home. It's a genuine part of the Nations present job to keep tight the bonds between its

fighters and those at home who can follow only with their hearts. Pictures from home and to the home can do their part. There's room for a little Vest Pocket Kodak in every sailor's and soldier's kit. There expense is small, six dollars. The cheerfulness it may bring is great.

Implied Audience: soldiers / families

Product: vest pocket Kodak

Photo or Illustration Based: illus

Color or Black and White: bw

Description / Notes: Illustration of a sailor holding a goat on board a ship, another sailor taking his photograph.

Binder: 1914-1916 5 of 5

Slogan: Snap-shots from Home.

Subject(s): soldiers

Text: Give cheer to the boys in camp and on shipboard by sending them pictures from home. There are likely to be some tedious, homesick days and a little cheer-up in the way of photographs of the home folks and the home doings will do them a lot of good. And some day when you want to give something a little more substantial, send along a vest pocket Kodak and ask your soldier or sailor boy to send pictures to you.

Implied Audience: soldiers / families

Product: vest pocket Kodak

Photo or Illustration Based: Illus

Color or Black and White: bw

Description / Notes: Illustration of a group of soldiers looking at photographs at a camp.
Binder: $1914-19165$ of 5

Slogan: The Parting Gift-- A Vest Pocket Kodak

Subject(s): woman / soldiers on a train

Text: It is monotony, not bullets that our soldier boys dread. No fear, when the time comes, they will uphold bravely the traditions that are dear to every loyal American heart. But in the training camps and during the months of forced inaction there are going to be some tedious, home-sick days-days the Kodak can make more cheerful. Pictures of comrades and camp life, pictures of the housand and one things that can be photographed without endangering any military secret will interest them, and will doubly interest the friends at home. Tens of thousands of brave lads in the camps and trenches of France are keekping their own Kodak Story of the war-a story that will always be intense to them because it is history from their view-point. And when peace comes it will make more vivid, more real their story of the war as they tell it again and again to mother and sister and wife and little ones. The nation has a big job on its hands. It's only a little part, perhaps, but a genuine part of that job to keep up the

cheerfullness of camp life, to keep tight the bonds between camp and home. Pictures from home to the camp and from the camp to the home can do their part. There's room for a little Vest Pocket Kodak in every soldier's and sailor's kit. Ther expense is small, six dollars. The cheerfullness it may bring is great.

Implied Audience: soldiers / families

Product: vest pocket Kodak

Photo or Illustration Based: illus

Color or Black and White: bw

Description / Notes: illustration of a woman handing a soldier on a train a camera. There is a second version with a more simple illustration.

Binder: $1918-1923$ General Ads 1 of 10

Year: 1918

Slogan: A Useful Booklet for the Roentgenologist

Subject(s): product - medical

Product: "X-Rays" Booklet

Color or Black and White: 
Binder: 1918-1923 General Ads 1 of 10

Year: May-18

Slogan: 2c Kodak Jr.

Subject(s): product - camera

Product: 2c Kodak Jr.

Color or Black and White: Publications: Century, Century Life, Harper, National Geographic, Popular Science, Scribners, Worlds

Work, Popular Mechanics, Red Cross, Life, National Sportsman

Description / Notes: Price: from $\$ 15.50$ to $\$ 23.50$

Binder: $1918-1923$ General Ads 1 of 10

Year: Jul-18

Slogan: Eastman Dental Film Tank

Subject(s): product - medica

Product: Eastman Dental X-Ray Film Tank

Color or Black and White:

Binder: 1918-1923 General Ads 1 of 10

Year: 1917

Slogan: Your Practice Demands Eastman Dental X-Ray Films

Subject(s): product - medical

Product: Eastman Dental X-Ray Films

Description / Notes: Caption reads: "Courtesy of Dr. S. M.

Getzoff

Binder: $1918-1923$ General Ads 1 of 10

Year: 1917

Slogan: The Determining Factor--Eastman Dental X-Ray Films

Subject(s): product - medical

Product: Eastman Dental X-Ray Films

Description / Notes: Caption reads: "Dr. Albert Kinley, Boston, Mass."

Binder: $1918-1923$ General Ads 1 of 10

Year: Feb-18

Slogan: Be Sure of Your Claims

Subject(s): product - medical

Product: Eastman Dental X-Ray Films

\section{Color or Black and White}

Binder: 1918-1923 General Ads 1 of 10

Year: 1918

Slogan: You Need Them

Subject(s): product - medical

Product: Eastman Dental X-Ray Films

Color or Black and White:

Binder: 1918-1923 General Ads 1 of 10 Year: May-18

Slogan: Causes and Effects

Subject(s): product - medica

Product: Eastman Dental X-Ray Films

Color or Black and White:

Binder: 1918-1923 General Ads 1 of 10

Year: Jun-18

Slogan: Eastman Dental X-Ray Films

Subject(s): product - medical

Product: Eastman Dental X-Ray Films

Color or Black and White:

Binder: $1918-1923$ General Ads 1 of 10 Year: 1918

Slogan: Judging by the Evidence

Subject(s): product - medical

Product: Eastman Dental X-Ray Films

Color or Black and White:

Binder: 1918-1923 General Ads 1 of 10

Year: 1918

Slogan: The Safest Record Eastman Dental X-Ray Films

Subject(s): product - medical

Product: Eastman Dental X-Ray Films

Color or Black and White:

Binder: 1918-1923 General Ads 1 of 10

Year: 1918 
Slogan: Put Your Patients on File.

Subject(s): product - medical

Product: Eastman Dental X-Ray Films

Color or Black and White:

Binder: 1918-1923 General Ads 1 of 10

Year: 1918

Slogan: Make your Diagnoses Permanent

Subject(s): product - medical

Product: Eastman Dental X-Ray Films

Color or Black and White:

Binder: 1918-1923 General Ads 1 of 10

Year: 1918

Slogan: Make Your Profession Tangible

Subject(s): product - medical

Product: Eastman Dental X-Ray Films

Color or Black and White:

Binder: $1918-1923$ General Ads 1 of 10

Year: Jul-18

Slogan: Introducing the new Eastman Dupli-Tized X-Ray Films

Subject(s): product - medical

Product: Eastman Dupli-tized X-Ray Films

Color or Black and White:

Binder: 1918-1923 General Ads 1 of 10

Year: 1918

Slogan: Eastman Dupli-Tized X-Ray Films

Subject(s): product - medical

Product: Eastman Dupli-tized X-Ray Films

Color or Black and White:

Binder: $1918-1923$ General Ads 1 of 10

Year: 1918

Slogan: Like Air Photography

Subject(s): product - medica

Product: Eastman Dupli-tized X-Ray Films

Color or Black and White:
Binder: 1918-1923 General Ads 1 of 10

Year: 1918

Slogan: When Speed is Essential

Subject(s): product - medical

Product: Eastman Dupli-tized X-Ray Films

Color or Black and White

Binder: 1918-1923 General Ads 1 of 10

Year: 1918

Slogan: Make Your Records Permanent, Use Eastman Duplitized

X-Ray Films

Subject(s): product - medical

Product: Eastman Dupli-tized X-Ray Films

Color or Black and White:

Binder: 1918-1923 General Ads 1 of 10

Year: 1918

Slogan: Eastman Film

Subject(s): product - film \& paper

Product: Eastman Films

Color or Black and White:

Binder: $1918-1923$ General Ads 1 of 10

Year: Jun-18

Slogan: Eastman X-Ray Developer Powders

Subject(s): product - medical

Product: Eastman X-Ray Developing Powders

Color or Black and White:

Binder: $1918-1923$ General Ads 1 of 10

Year: 1918

Slogan: Eastman X-Ray Films

Subject(s): product - medical

Product: Eastman X-Ray Films

Color or Black and White:

Binder: 1918-1923 General Ads 1 of 10

Year: Apr-18 
Slogan: There's the Evidence

Subject(s): product - medical

Product: Eastman X-Ray Films

Color or Black and White:

Binder: 1918-1923 General Ads 1 of 10

Year: Jul-18

Slogan: Let the Children Kodak

Subject(s): Child with animals

Text: none

Implied Audience: families

Product: Kodak

Photo or Illustration Based: photo

Color or Black and White: bw

Description / Notes: Photograph of a boy at a zoo photographing an elk.

Binder: 1918-1923 General Ads 1 of 10

Year: May-18

Slogan: Prove it--with a Kodak

Subject(s): children

Text: Every kind of fun when there's a Kodak to keep the picture

story. Every negative can carry the date and title, made on the

film at the time. It's all very simple by the Kodak system.

Implied Audience: parents/children

Product: Kodak

Photo or Illustration Based: photo

Color or Black and White: bw

Description / Notes: Photograph of three children playing by the side of a lake.

Binder: $1918-1923$ General Ads 1 of 10

Slogan: All Out-doors invites your Kodak

Subject(s): children

Text: none

Implied Audience: parents/children

Product: Kodak

Photo or Illustration Based: photo

Color or Black and White: bw
Description / Notes: Photograph of two boys by a tree, looking at a camera.

Binder: 1918-1923 General Ads 1 of 10

Year: Jul-18

Slogan: Take a Kodak with You

Subject(s): hunter

Text: none

Implied Audience: outdoorsmen

Product: Kodak

Photo or Illustration Based: photo

Color or Black and White: bw

Publications: Field and Stream,

Description / Notes: Photograph of a man with a rifle, taking a photograph of something.

Binder: $1918-1923$ General Ads 1 of 10

Slogan: Take a Kodak with You

Subject(s): Kodak Girl

Text: none

Implied Audience: Women

Product: Kodak

Photo or Illustration Based: photo

Color or Black and White: bw

Description / Notes: Photograph of a woman with her Kodak in a

fur coat and hat, leaning on a car.

Binder: 1918-1923 General Ads 1 of 10

Slogan: All Out-doors invites your Kodak

Subject(s): Kodak Girl

Text: none

Implied Audience: women / general

Product: Kodak

Photo or Illustration Based: Photo

Color or Black and White: bw

Description / Notes: Photograph of a woman on a mountainside

with snowshoes in her hand, Kodak slung over her shoulder.

Binder: 1918-1923 General Ads 1 of 10 
Year: May-18

Slogan: Keep a Kodak story of the Children for the children

Subject(s): mother / young girl

Text: none

Implied Audience: families

Product: Kodak

Photo or Illustration Based: photo

Color or Black and White: bw

Description / Notes: Photograph of a mother and young girl

looking at an album.

Binder: 1918-1923 General Ads 1 of 10

Slogan: The Kodak Album

Subject(s): mother / young gir

Text: All the little kings and queens of childhood's realm command your Kodak--command it to keep their picture story. Pictures of them and by them, pictures of their friends and their pranks-pictures that breathe the very joy and frankness of childhood. Such is the intimate picture story that makes the Kodak Album the most cherished book in all the house. And picture taking is very simple now. Kodak has made it so.

Implied Audience: families

Product: Kodak

Photo or Illustration Based: photo

Color or Black and White: bw

Description / Notes: Photograph of a mother and young girl

looking at an album--same as above but with different text.

Binder: 1918-1923 General Ads 1 of 10

Slogan: There's a photographer in your town

Subject(s): none

Text: The pictures you are planning to send to that Soldier of yours--they must soon be on the way if you would make sure that he has them to gladden his heart on Christmas morning.

Implied Audience: soldiers / families

Product: Kodak

Photo or Illustration Based: photo

Color or Black and White: bw

Description / Notes: no photo- text only.
Binder: 1918-1923 General Ads 1 of 10

Year: Mar-18

Slogan: The Pictures from Home

Subject(s): soldier

Text: Keep your Kodak busy for the sake of the boys "over there."

Implied Audience: soldier

Product: Kodak

Photo or Illustration Based: photo

Color or Black and White: bw

Description / Notes: Photograph of a soldier sitting in his tent -

the same image is used in different ads.

Binder: $1918-1923$ General Ads 1 of 10

Year: May-18

Slogan: The Days of His Going

Subject(s): Soldier / parents

Text: In a million homes, pictures are keeping the story of the war as it touched those homes. John in his first khaki as he proudly marched away, and John tanned and hardened as he looked when home on leave. More than ever the Kodak album is keeping the home story. To-day that story means history, and more than ever it is important that it be authentic history--that every negative bear a date. Memory plays strange tricks and one of its favorite vagaries is to fail in the all important matter of dates. But with a Kodak there's no uncertainty. The date--and title too, if you wish-is written on the autographic film at the time the exposure is made. And it is there permanently. It makes the Kodak story authentic and doubly interesting. It is all very simple, is the work of an instant and there's no extra charge for autographic film. Let the Kodak keep the dates.

Implied Audience: soldiers / families

Product: Kodak

Photo or Illustration Based: photo

Color or Black and White: bw

Publications: American, Motion Picture Way, Cosmopolitan,

Country Gentleman, Saturday Evening Post, Life, Mumseys,

Popular Science.

Description / Notes: photograph of a soldier, holding a young 
girl while the mother writes on the camera.

Binder: 1918-1923 General Ads 1 of 10

Slogan: Those pictures from home

Subject(s): soldiers

Text: "If they only knew the pleasure they bring us I am sure they would come" .... Thus writes Gustave Geboers a Belgian boy of twenty three. And your boy, our boys, will pictures mean less to

them?...

Implied Audience: soldiers / families

Product: Kodak

Photo or Illustration Based: photo/Text: Color or Black and

White: bw

Description / Notes: extracts from a letter. Caption identifies his position in the Belgian army, and there is the French writing as well.

Binder: 1918-1923 General Ads 1 of 10

Slogan: There's cheer in the pictures from home.

Subject(s): soldiers

Text: To a homesick boy at the front, a picture of Dad waiting at the end of the lane wile "Shep" brings in the cows is worth more than the Croix de Guerre. Pictures of mother, how much they mean to him now! And of his kid sister perhaps she is "wearing her hair up" by this time--all the old familiar scenes around the farm, yes, and that little girl with the big blue eyes that lives down in the village--these will mean a world of comfort to the boy who is lonesome among a million strangers. The YMCA the Red Cross, the Knights of Columbus and kindred organizations are doing a world of good in ministering to the bodies and the minds of our boys. But in their hearts, homes are first. Cheerful letters and cheerful pictures from home--these will keep their hearts light and their courage high.

Implied Audience: soldiers / families

Product: Kodak

Photo or Illustration Based: illustration

Color or Black and White: bw

Description / Notes: Illustration of soldiers outside the YMCA looking at photographs.
Binder: 1918-1923 General Ads 1 of 10

Year: 1917

Slogan: "Kodak"

Subject(s): product - misc.

Product: Kodak Branding

Description / Notes: Trademark \& Copyright info.

Binder: $1918-1923$ General Ads 1 of 10

Year: 1917

Slogan: If it isn't an Eastman, it isn't a Kodak.

Subject(s): product - misc.

Product: Kodak Branding

Description / Notes: Trademark \& Copyright info.

Binder: 1918-1923 General Ads 1 of 10

Year: Jan-18

Slogan: No. 1a Autographic Kodak Special with Kodak Range Finder

Subject(s): product - camera

Product: No. 1a Autographic Kodak Special

Description / Notes: Price: from $\$ 50.00$ to $\$ 65.00$

Binder: 1918-1923 General Ads 1 of 10

Year: Jun-18

Slogan: All Outdoors invites your Kodak

Subject(s): man in a canoe

Text: In the woods where the big game hides, in every marshy

haunt of the swift-winged duck, pictures are awaiting you. In the cover where your good dog noses out the quail; in the pool where King Trout is waiting to fool you, if he can; and by the little lake where lurk the gamey bass and mighty muskalonge, there are pictures--waiting for you and your Kodak. Accept, on behalf of yourself and your Kodak, Nature's urgent invitation. And next winter, when business and snow and ice conspire to keep you in, those pictures will again be waiting for you--secure within the pages of your Kodak Album.

Implied Audience: outdoorsmen

Product: photograph 
Photo or Illustration Based: photo

Color or Black and White: bw

Description / Notes: Photograph of a man in a canoe with a Kodak photographing birds eggs.

Binder: 1918-1923 General Ads 1 of 10

Slogan: There's a photographer in your town

Subject(s): none

Text: That lad of yours, over seas. All that is humanly possible is being done to see to it that he is well equipped. Organizations like the YMCA, are looking to his physical comfort, healthful recreation and clean fun. If he is sick or wounded the Red Cross will provide for him with tender, loving care. Yet there is one thing that will bring a smile to his face and joy to his heart that none of these can give; that only you can give--your photograph.

Implied Audience: soldiers / families

Product: photograph

Photo or Illustration Based: Text: Color or Black and White: bw

Publications: Century, Country life, Harpers, National

Geographic, Scribners, Popular Mechanics, Red Cross.

Description / Notes: no photo- text only.

Binder: 1918-1923 General Ads 1 of 10

Year: 1918

Slogan: Those Pictures from Home

Subject(s): product - misc.

Product: photograph

Color or Black and White:

Binder: $1918-1923$ General Ads 1 of 10

Year: Jun-18

Slogan: Before He Goes

Subject(s): Soldier / family

Text: When we have won this war--when our boys sail home across seas forever freed from pirates submarines--when our boys, bronzed and sinewy, hardened veterans all, swing with martial, ringing tread past the reviewing stands, victors in the battle for the democracy;--first in their heartsand minds will the thoughts of home. Mustered out--overwhelmed with the acclaim of a nation-they go back to the brave homes so bravely defended. Happily, they take up the pursuits of peace--but never will they will to forget that they have been soldiers for democracy. And they will always be grateful for that which will keep fresh in their memories the story of the Great War. This thing pictures can do. Pictures of the parting and of the home coming, pictures of comrades in arms, pictures that tell intimetly of how the war touched their lives.

These will have value beyond price. And while they are still in camp, are patrolling the high seas, or battering the Teuton line in France, pictures of their home folks and home doings, Kodak pictures such as you can take will help to "Turn the Dark cloud inside out, till the boys come home."

Implied Audience: soldiers / families

Product: photographs

Photo or Illustration Based: photo

Color or Black and White: bw

Publications: McClures

Description / Notes: Photograph of a woman photographing a soldier holding his child.

Binder: 1918-1923 General Ads 1 of 10

Year: 1918

Slogan: Pictures from Home Maintain the Morale

Subject(s): product - misc.

Product: photographs

Color or Black and White:

Binder: $1918-1923$ General Ads 1 of 10

Year: Nov-17

Slogan: Seed X-Ray Plates

Subject(s): product - medical

Product: Seed X-Ray Plate

Description / Notes: Caption reads: "Seed Stereoscopic Chest Plate"

Binder: $1918-1923$ General Ads 1 of 10

Year: 1917

Slogan: $100 \%$ Increase in the use of Seed X-Ray Plates 
Subject(s): product - medical

Product: Seed X-Ray Plate

Color or Black and White:

Binder: $1918-1923$ General Ads 1 of 10

Year: 1918

Slogan: The Special Kodaks

Subject(s): product - camera

Product: Special Kodaks

Color or Black and White:

Binder: $1918-1923$ General Ads 10 of 10

Year: Jan-23

Slogan: Kodak welcomes Winter

Subject(s): Kodak Girl

Text: There's a tang to the air, and a zest to the occasion that

give life and action to the pictures you make. Winter prints

contribute prized pages to your album. And it's all easy the Kodak way--and all fun.

Implied Audience: outdoors / women

Product: autographic Kodak

Photo or Illustration Based: photo

Color or Black and White: bw

Description / Notes: Photo of a woman photographing a horsedrawn sleigh full of people.

Binder: 1918-1923 General Ads 10 of 10

Year: Jan-23

Slogan: All Out-doors invites your Kodak

Subject(s): Kodak Girl

Text: none

Implied Audience: women / travelers

Product: autographic Kodak

Photo or Illustration Based: photo

Color or Black and White: bw

Description / Notes: Photo of a woman on the ice waving at the

camera, a man with a sailboat in the bg.

Binder: 1918-1923 General Ads 10 of 10
Year: Jan-23

Slogan: A Kodak for the Farm

Subject(s): Pigs

Text: It's easy to make pictures the Kodak way, and the resulting prints of livestock, buildings, crops, and equipment, whether for purposes of sale or record, are of obvious value. There's fun with a Kodak for everyone--and growing pleasure in the pictures, but on the farm photography has a practical side that should not be overlooked.

Implied Audience: farmers

Product: autographic Kodak

Photo or Illustration Based: photo

Color or Black and White: bw

Description / Notes: photo of a lot of pigs eating at a trough.

Caption reads "From a Kodak negative."

Binder: $1918-1923$ General Ads 10 of 10

Slogan: Her Christmas Kodak

Subject(s): young man, woman

Text: Out of the holiday package into the spirit of Christmas

Implied Audience: Christmas

Product: autographic Kodak

Photo or Illustration Based: photo

Color or Black and White: bw

Description / Notes: Photo of a woman holding a Kodak while

man watches. Christmas gifts are on the table.

Binder: $1918-1923$ General Ads 10 of 10

Slogan: Her Christmas Kodak

Subject(s): Young woman

Text: None

Implied Audience: women / Christmas

Product: autographic Kodak / brownies

Photo or Illustration Based: photo

Color or Black and White: bw

Description / Notes: Photo of a young woman standing at a

front door holding a Kodak and a pile of Christmas gifts.

Binder: 1918-1923 General Ads 10 of 10 
Slogan: A Brownie for Christmas

Subject(s): boy and girl

Text: Means pictures of your friends, your sports, your pets-- of all the things that interest you. There's fun in photography and pride in the kind of pictures a Brownie makes. It's all easy--dead easy--from the start, with this Eastman-made camera. You'll get good pictures on Christmas day.

Implied Audience: parents/children/Christmas

Product: Brownie

\section{Photo or Illustration Based: photo}

Color or Black and White: bw

Description / Notes: photo of a boy photographing his sister and a sled on their front yard, in the snow.

Binder: 1918-1923 General Ads 10 of 10

Slogan: Bring Along A Brownie

Subject(s): three boys

Text: Making pictures--that's fun. Looking at them afterwards and showing them to your friends and family--that's still more fun as you'll find from the first. And it's all easy with a Brownie.

Implied Audience: parents/children

Product: Brownie

Photo or Illustration Based: photo

Color or Black and White: bw

Description / Notes: Photo of three boys on a fallen tree near

the water, one holding a fishing rod, one holding a Brownie and photographing the other boy holding a fish.

Binder: 1918-1923 General Ads 10 of 10

Slogan: Get it with a Brownie

Subject(s): three boys

Text: The shack that the bunch built offers a great chance for a picture. Get it with a Brownie. Cross country hikes, sports of all sorts--your friends, your pets--all call for pictures that are easy to make the Brownie way. No matter what the occasion there's always more fun with a Brownie along.

Implied Audience: parents/children

Product: Brownie

Photo or Illustration Based: photo
Color or Black and White: bw

Description / Notes: Photograph of a boy photographing two other boys building a shack out of branches.

Binder: 1918-1923 General Ads 10 of 10

Year: 1923

Slogan: Eastman Dental Film Mount

Subject(s): product - medical

Product: Eastman Dental Film Mount

Color or Black and White

Binder: $1918-1923$ General Ads 10 of 10

Year: 1922

Slogan: For your Convenience an Eastman Dental Film Safe.

Subject(s): product - medical

Product: Eastman Dental Film Safe

Description / Notes: price: $\$ 3.00$

Binder: $1918-1923$ General Ads 10 of 10

Year: 1922

Slogan: The great advantage of Eastman Dupli-Tized Film

Subject(s): product - film \& paper

Product: Eastman Dupli-tized X-Ray Films

Color or Black and White:

Binder: $1918-1923$ General Ads 10 of 10

Year: 1923

Slogan: To Certify the Inter-Oral Set: A Stereo Pair--Extra Orally

Subject(s): product - medical

Product: Eastman Dupli-tized X-Ray Films

Color or Black and White:

Binder: $1918-1923$ General Ads 10 of 10

Year: 1922

Slogan: Eastman Organic Chemicals - List No. 8 America's

Handbook of Organic Chemicals

Subject(s): product - Chemicals

Product: Eastman Organic Chemicals

Color or Black and White: 
Binder: $1918-1923$ General Ads 10 of 10

Year: 1923

Slogan: Eastman Organic Chemicals - Over 1400 Available.

Subject(s): product - Chemicals

Product: Eastman Organic Chemicals

Color or Black and White:

Binder: $1918-1923$ General Ads 10 of 10

Year: 1923

Slogan: Eastman Organic Chemicals - Quality Unsurpassed

Subject(s): product - Chemicals

Product: Eastman Organic Chemicals

Color or Black and White:

Binder: $1918-1923$ General Ads 10 of 10

Year: Jan-23

Slogan: Eastman Organic Chemicals - Over 1500 Available

Subject(s): product - Chemicals

Product: Eastman Organic Chemicals

Color or Black and White:

Binder: 1918-1923 General Ads 10 of 10

Year: 1922

Slogan: Announcing Eastman X-Ray Intensifying Screens

Subject(s): product - medical

Product: Eastman X-Ray Intensifying Screens

Description / Notes: Price from $\$ 8.00$ to $\$ 24.00$ each. $\$ 15.00$ to $\$ 45.00$ for pairs.

Binder: 1918-1923 General Ads 10 of 10

Year: 1923

Slogan: Eastman X-Ray Intensifying Screens

Subject(s): product - medical

Product: Eastman X-Ray Intensifying Screens

Color or Black and White:

Binder: 1918-1923 General Ads 10 of 10

Year: 1923
Slogan: For Efficiency, Speed, Quality, and Uniformity

Subject(s): product - medical

Product: Eastman X-Ray Intensifying Screens / Eastman Dupli-

Tized X-Ray Films

Color or Black and White:

Binder: $1918-1923$ General Ads 10 of 10

Year: 1922

Slogan: A New model Kodak--and just in time for Christmas No. 1

Pocket Kodak Series II

Subject(s): product - camera

Product: No. 1 Pocket Kodak Series II

Description / Notes: Price: $\$ 20.00$

Binder: $1918-1923$ General Ads 10 of 10

Year: 1922

Slogan: Kodak pictures prove your selling arguments

Subject(s): product - camera

Product: No. 2c Autographic Kodak, Jr.

Description / Notes: Price: $\$ 50.00$. Caption reads "From a Kodak negative"

Binder: 1918-1923 General Ads 10 of 10

Slogan: There's a photographer in your town

Subject(s): none

Text: This Christmas your photograph--the only gift that only you can make. And now is the time for a studio appointment.

Implied Audience: Christmas

Product: photograph

Photo or Illustration Based: Text: Color or Black and White: bw

Description / Notes: No photo - text only.

Binder: 1918-1923 General Ads 10 of 10

Year: Jan-23

Slogan: Photographing Five Miles

Subject(s): product - camera

Product: The Kodak Anastigmats

Color or Black and White: 
Binder: 1918-1923 General Ads 2 of 10

Year: 1922

Slogan: His First Dress Suit

Subject(s): family

Text: Bill Jr. is conscious of a newly acquired dignity; little Jim is envious; Mother sits in rapt admiration; Betty has an attack of the giggles, and Dad, in spite of his sense of humor, realizes that this is a great day in the history of his son and heir. Faithful to his Kodak habit, he has taken a picture of the boy in his first dress suit--and now he is about to jot down the date on the autographic film. In just a few years when he and Son are looking at the family Kodak album, he will smile slyly and say--"William you were great! Let's see, how old were you then?" And the autographic film will

tell.

Implied Audience: families

Product: autographic Kodak

Photo or Illustration Based: photo

Color or Black and White: bw

Description / Notes: Photograph of a family in the parlor, looking at the oldest boy in a new suit.

Binder: 1918-1923 General Ads 2 of 10

Year: 1919

Slogan: For the Days to Come

Subject(s): father / son

Text: Building his boat of pine and dreaming, as he works, of the days when he will sail a real ship on a real ocean--a regular boy, that. And Dad, with his Kodak, has caught the boyish story. Now he is writing the autographic record--the date and title on the film; the record that will give double value to the picture when time has played sad tricks with memory. Make the family chronicle complete. Let every picture of the children bear at least a date. It's all very simple, as simple as taking the picture itself--with an Autographic Kodak

Implied Audience: families

Product: autographic Kodak

Photo or Illustration Based: photo

Color or Black and White: bw
Description / Notes: photograph of a father with a camera photographing his son playing with a boat.

Binder: 1918-1923 General Ads 2 of 10

Year: Jun-19

Slogan: The Day that Will Never Come Again

Subject(s): Man and woman watching soldier's parade.

Text: The boys are home. Eyes front and chins up, crusaders all, hardened veterans in a glorious cause they tramp past your point of vantage. Your heart becomes a trip hammer; you join wildly in the cheers; you thrill with a great love; a love of country and a love for the men who have saved the world. This day marks an epoch whose events you are privileged to witness; it is a day that will never come again. It is all worth far more than merely seeing-it is worth saving. Pictures from your point of vantage--just as you saw it--that recall the glory and the greatness of it all these will keep it fresh and vivid, will bring back the thrill and breathless glow even when memory alone can scarce recall the scene. And on each negative you may have, not merely the picture story, but the date and title, the full authentic history--with an autographic

Kodak.

Implied Audience: soldiers

Product: autographic Kodak

Photo or Illustration Based: photo

Color or Black and White: bw

Description / Notes: illustration of a woman inscribing on the camera, while a man watches. Outside, soldiers are parading by.

Binder: 1918-1923 General Ads 2 of 10

Year: 1919

Slogan: Keep a Kodak Story of the Children

Subject(s): mother / young girl

Text: And keep, as well the date on the film, the authentic record that tells how old Mary was when she took her first toddling steps and the year and month when "brother" proudly donned his first trousers, or bravely set out for school with primer under his arm. Such records are instantly and easily made and permanently kept with an Autographic Kodak.

Implied Audience: families 
Product: autographic Kodak

Photo or Illustration Based: photo?

Color or Black and White: bw

Description / Notes: photograph of a woman photographing her young daughter who is blowing bubbles.

Binder: 1918-1923 General Ads 2 of 10

Year: 1919

Slogan: Keep a Kodak Story of the Children

Subject(s): mother and two young children

Text: In every day of their young lives are events of almost

dramatic interest: The painted gallop across the porch on the hobb

horse; the adventure with the puppy in the garden; sister's new

frock and brother's tricycle; that important morning when stout

hearts first trudge off to school--such pictures, preserving forever the childhood days, mean a world of comfort to mother's heart-yes, and fathers too. And just a few years afterward: "That's you, Polly, when you were--let me see. Oh yes, the film says it was August 8, 1919, your fourth birthday. And Junior was five." Every picture worth taking is worth at least a date, if not a title. It's all very simple with an Autographic Kodak, as simple as pressing a button. And Autographic film costs no more than the other kind.

Implied Audience: families

Product: autographic Kodak

Photo or Illustration Based: photo

Color or Black and White: bw

Description / Notes: Photograph of a mother with a camera, photographing her two young children at a gate.

Binder: 1918-1923 General Ads 2 of 10

Year: 1919

Slogan: The Date on the Film. $\mathrm{N}$ just a few years you will ask:

Subject(s): product - camera

Product: Autographic Kodak

Color or Black and White:

Binder: 1918-1923 General Ads 2 of 10

Slogan: Let the Children Kodak

Subject(s): two young girls
Text: And get them to keep, on every film, the date and title that add so much in interest. It's all very simple with an Autographic Kodak of Brownie--and all folding Kodaks and folding Brownies are now autographic.

Implied Audience: parents.

Product: autographic Kodak / brownies

Photo or Illustration Based: photo

Color or Black and White: bw

Description / Notes: Photograph of two girls on the seaside, writing with an Autographic Kodak.

Binder: 1918-1923 General Ads 2 of 10

Slogan: Every Sport is more Sport with a Brownie along.

Subject(s): boys fishing with dog

Text: none

Implied Audience: parents/children

Product: Brownie

Photo or Illustration Based: photo

Color or Black and White: bw

Description / Notes: Photograph of two young boys fishing. One boy holds a rod, the other holds a brownie, there is a dog sitting between them.

Binder: 1918-1923 General Ads 2 of 10

Year: 1919

Slogan: Keep a File Record. Eastman Dental X-Ray Films

Subject(s): product - medical

Product: Eastman Dental X-Ray Films

Color or Black and White:

Binder: 1918-1923 General Ads 2 of 10

Year: 1919

Slogan: Give yourself assurance. Eastman Dental X-Ray Films

Subject(s): product - medical

Product: Eastman Dental X-Ray Films

Color or Black and White:

Binder: 1918-1923 General Ads 2 of 10

Year: 1919 
Slogan: X-Ray Diagnosis.

Subject(s): product - medical

Product: Eastman Dental X-Ray Films

Color or Black and White:

Binder: 1918-1923 General Ads 2 of 10

Year: 1919

Slogan: Keep pace with the times.

Subject(s): product - medical

Product: Eastman Dental X-Ray Films

Color or Black and White:

Binder: 1918-1923 General Ads 2 of 10

Year: 1919

Slogan: You know their value. Eastman Dental X-Ray Films.

Subject(s): product - medical

Product: Eastman Dental X-Ray Films

Color or Black and White:

Binder: 1918-1923 General Ads 2 of 10

Year: 1919

Slogan: Do The Obvious Thing. Eastman Dental X-Ray Films

Subject(s): product - medical

Product: Eastman Dental X-Ray Films

Color or Black and White:

Binder: 1918-1923 General Ads 2 of 10

Year: 1919

Slogan: Put your patients on record

Subject(s): product - medical

Product: Eastman Dental X-Ray Films

Color or Black and White:

Binder: 1918-1923 General Ads 2 of 10

Year: 1919

Slogan: The Business-like way. Eastman Dental X-Ray Films

Subject(s): product - medical

Product: Eastman Dental X-Ray Films

Color or Black and White:
Binder: $1918-1923$ General Ads 2 of 10

Year: 1919

Slogan: Keep Pace with the Times. Eastman Dental X-Ray Films

Subject(s): product - medical

Product: Eastman Dental X-Ray Films

Color or Black and White:

Binder: $1918-1923$ General Ads 2 of 10

Year: 1919

Slogan: Efficiency plus, with Eastman Dupli-Tized X-Ray Films

Subject(s): product - medical

Product: Eastman Dupli-tized X-Ray Films

Description / Notes: Caption Reads: "Two Screen Portion / One

Screen Portion"

Binder: 1918-1923 General Ads 2 of 10

Year: 1919

Slogan: That important case may depend on Eastman Dupli-Tized

X-Ray films

Subject(s): product - medical

Product: Eastman Dupli-tized X-Ray Films

Color or Black and White:

Binder: $1918-1923$ General Ads 2 of 10

Year: 1919

Slogan: Eastman Dupli-Tized X-Ray Films

Subject(s): product - medical

Product: Eastman Dupli-tized X-Ray Films

Color or Black and White:

Binder: 1918-1923 General Ads 2 of 10

Year: 1919

Slogan: For Gastro-Intestinal Diagnoses. Eastman Dupli-Tized X-

Ray Films

Subject(s): product - medical

Product: Eastman Dupli-tized X-Ray Films

Color or Black and White: 
Binder: 1918-1923 General Ads 2 of 10

Year: 1919

Slogan: For Stereoscopy: Eastman Duplitized X-Ray Film

Subject(s): product - medical

Product: Eastman Dupli-tized X-Ray Films

Color or Black and White:

Binder: 1918-1923 General Ads 2 of 10

Year: 1919

Slogan: A Shrapnel Wound shown by Eastman Dupli-Tized X-Ray

Film

Subject(s): product - medical

Product: Eastman Dupli-tized X-Ray Films

Color or Black and White:

Binder: 1918-1923 General Ads 2 of 10

Year: 1919

Slogan: Gastro-Intestinal Roentgenology with Eastman Dupli-

Tized Films

Subject(s): product - medical

Product: Eastman Dupli-tized X-Ray Films

Description / Notes: Caption on image gives info about

exposure, method, etc.

Binder: 1918-1923 General Ads 2 of 10

Year: 1919

Slogan: The New Package

Subject(s): product - medical

Product: Eastman Dupli-tized X-Ray Films

Color or Black and White:

Binder: 1918-1923 General Ads 2 of 10

Year: 1919

Slogan: Eastman Organic Chemicals

Subject(s): product - Chemicals

Product: Eastman Organic Chemicals

Color or Black and White:

Binder: 1918-1923 General Ads 2 of 10
Year: 1919

Slogan: Eastman Organic Chemicals

Subject(s): product - Chemicals

Product: Eastman Organic Chemicals

Color or Black and White:

Binder: 1918-1923 General Ads 2 of 10

Slogan: A Kodak Christmas

Subject(s): Family

Text: Kodak helps to make the Christmas Merry--then keeps a picture story of the Christmas Merriment. And nowhere are there more Christmas pictures for the Kodak than in the country where Christmas is so real, so home like, so full of the old-fashioned, whole souled spirit of the day. The arrival of the visitors; the children romping in the snow, the gorgeous sun and snow effects in the orchard... All these are subjects for the Kodak. In taking and titling the pictures there is fun and interest that give an added zest to the Christmas merry-making--and afterwards these pictures, telling so faithfully the home story, help to keep fresh the

memories of the great home holiday. Put Kodak on your Christmas list.

Implied Audience: families

Product: Kodak

Photo or Illustration Based: illus

Color or Black and White: bw

Description / Notes: Illustration of a man, woman, and baby arriving at a home in a car, while older parents wait at the house door, and a girl photographs the arrival.

Binder: 1918-1923 General Ads 2 of 10

Slogan: Kodak

Subject(s): Kodak Girl

Text: The gift that helps to make her Christmas merry--then

keeps a picture story of the Christmas merriment.

Implied Audience: women / Christmas

Product: Kodak

Photo or Illustration Based: photo

Color or Black and White: bw

Description / Notes: photograph of a woman holding a Kodak in 
a fur coat and hat, holding a bouquet of holly.

Binder: 1918-1923 General Ads 2 of 10

Slogan: Keep a Kodak Story of the Children

Subject(s): mother / young girl

Text: none

Implied Audience: families

Product: Kodak

Photo or Illustration Based: photo

Color or Black and White: bw

Description / Notes: photograph of a mother, writing on an

autographic Kodak while daughter holds a toy. They are sitting outside.

Binder: 1918-1923 General Ads 2 of 10

Slogan: Along with your other fun--A Kodak

Subject(s): two young boys

Text: Kodak adds to the sport on every outing. At the shore, hunting, camping, fishing--there are always pictures worth while-pictures you will be glad you have next winter and in all winters to come.

Implied Audience: parents/children

Product: Kodak

Photo or Illustration Based: photo

Color or Black and White: bw

Description / Notes: Photograph of two boys on the shore, one with a Kodak.

Binder: 1918-1923 General Ads 2 of 10

Slogan: Your First Dress Suit

Subject(s): Young man / family

Text: It is a big event in your life--but it is only one of many things that are happening to you every week. Years from now you would give anything to recall these days. You know what to do: Kodak as you go. Take pictures of your good times. Photograph your friends at every party or picnic. Snap the scenes of your trips and outings. Picture your big fish, your camp, your den, your cave or fort, .....everything that means fun to you now. With a Kodak you can write the date on the film when you take the picture.
Then--years from now--it will all come back to you and mean more then it does now. Ask your Dad if he doesn't think a Kodak would make a good Christmas present for a young man your age.

Implied Audience: families

Product: Kodak

Photo or Illustration Based: photo

Color or Black and White: bw

Description / Notes: Photograph of a family in the parlor,

looking at the oldest boy in a new suit - Same photograph as used above, but with different text.

Binder: $1918-1923$ General Ads 2 of 10

Year: 1922

Slogan: The Kodak Album

Subject(s): Group of girls

Text: There's joy in every page of the school girl's Kodak book.

There's a hint of good times in every tiny print and between its

covers is the pictured story of happy years and enduring

friendships--a story that means fun in the making, and afterward

the still greater joy of living over again those happy, care-free

days.

Implied Audience: parents/girls

Product: Kodak album

Photo or Illustration Based: photo

Color or Black and White: bw

Description / Notes: Photograph of a group of teenage girls

looking at an album.

Binder: $1918-1923$ General Ads 2 of 10

Year: 1919

Slogan: If it isn't an Eastman, it isn't a Kodak.

Subject(s): product - misc.

Product: Kodak Branding

Description / Notes: Info on Trademark. Includes a definition of the word "trademark"

Binder: 1918-1923 General Ads 2 of 10

Slogan: The Kodak Film

Subject(s): mother / young girl 
Text: And beneath each negative the date and title--the autographic record that makes the story, complete, authentic. Implied Audience: families

Product: Kodak film

Photo or Illustration Based: photo

Color or Black and White: bw

Description / Notes: Photograph of a mother holding a film roll, while daughter watches.

Binder: 1918-1923 General Ads 2 of 10

Year: 1919

Slogan: The Advantages of Eastman

Subject(s): product - film \& paper

Product: Motion Picture Film

Color or Black and White:

Binder: 1918-1923 General Ads 2 of 10

Slogan: There's a photographer in your town

Subject(s): none

Text: Between Friends--a photograph.

Product: photographs

Photo or Illustration Based: Text: Color or Black and White: bw

Description / Notes: no photo- text only.

Binder: 1918-1923 General Ads 2 of 10

Slogan: There's a photographer in your town

Subject(s): none

Text: At Christmas Time. Your friends can buy anything you can give them--except your photograph.

Product: photographs

Photo or Illustration Based: Text: Color or Black and White: bw

Description / Notes: no photo- text only.

Binder: 1918-1923 General Ads 2 of 10

Year: 1919

Slogan: The Picture Story, with the all important date and title on every negative is easy to keep with a Vest Pocket Autographic
Kodak

Subject(s): product - camera

Product: Vest Pocket Autographic Kodak

Color or Black and White:

Binder: 1918-1923 General Ads 3 of 10

Year: 1920

Slogan: It doubles the fun with a Brownie along.

Subject(s): young girl and boy

Text: none

Implied Audience: parents/children

Product: Brownie

Photo or Illustration Based: photo

Color or Black and White: bw

Description / Notes: Photograph of a boy with a bicycle, his sister taking a photograph of him with a Brownie.

Binder: 1918-1923 General Ads 3 of 10

Year: 1920

Slogan: Your Responsibility does not end. Eastman Dental X-Ray

Films

Subject(s): product - medical

Product: Eastman Dental X-Ray Films

Color or Black and White:

Binder: 1918-1923 General Ads 3 of 10

Year: 1920

Slogan: An Impacted Third Molar

Subject(s): product - medical

Product: Eastman Dental X-Ray Films

Color or Black and White:

Binder: 1918-1923 General Ads 3 of 10

Year: 1920

Slogan: Speed with Quality. Eastman Dental X-Ray Films

Subject(s): product - medical

Product: Eastman Dental X-Ray Films

Color or Black and White: 
Binder: 1918-1923 General Ads 3 of 10

Year: 1920

Slogan: For the Bedside Case

Subject(s): product - medica

Product: Eastman Dupli-tized X-Ray Films

Color or Black and White:

Binder: $1918-1923$ General Ads 3 of 10

Year: 1920

Slogan: Simplify the Difficult Dental Case with Eastman Dupli-

Tized X-Ray Films used Extra-Orally.

Subject(s): product - medical

Product: Eastman Dupli-tized X-Ray Films

Color or Black and White:

Binder: 1918-1923 General Ads 3 of 10

Year: 1920

Slogan: For Kidney and Gall-Bladder Technique Eastman Dupli-

Tized X-Ray Films with Double Screens

Subject(s): product - medica

Product: Eastman Dupli-tized X-Ray Films

Color or Black and White:

Binder: 1918-1923 General Ads 3 of 10

Year: 1920

Slogan: Eastman Organic Chemicals

Subject(s): product - Chemicals

Product: Eastman Organic Chemicals

Color or Black and White:

Binder: 1918-1923 General Ads 3 of 10

Year: 1920

Slogan: New Price List. Eastman Organic Chemicals

Subject(s): product - Chemicals

Product: Eastman Organic Chemicals

Color or Black and White:

Binder: 1918-1923 General Ads 3 of 10

Year: 1920
Slogan: The Eastman X-Ray Exposure Rule

Subject(s): product - medical

Product: Eastman X-Ray Exposure Rule.

Color or Black and White:

Binder: 1918-1923 General Ads 3 of 10

Slogan: Proof Positive

Subject(s): Fisherman posing with a fish.

Text: none

Implied Audience: outdoorsmen

Product: Kodak

Photo or Illustration Based: photo

Color or Black and White: bw

Description / Notes: Photo of three fishermen posing with a fish, while a fourth holds a Kodak, takes a photograph of them.

Binder: 1918-1923 General Ads 3 of 10

Slogan: Take a Kodak with You

Subject(s): landscape - mountains

Text: none

Implied Audience: travel

Product: Kodak

Photo or Illustration Based: photo

Color or Black and White: bw

Description / Notes: Photograph of a mountain range, lake,

trees. Caption reads: From a Kodak Negative.

Binder: $1918-1923$ General Ads 3 of 10

Year: 1920

Slogan: Kodak as you Go

Subject(s): Man and woman

Text: none

Implied Audience: travelers

Product: Kodak

Photo or Illustration Based: photo

Color or Black and White: bw

Description / Notes: Photograph of a man and woman at a

baggage check, the man handing the woman the Kodak. While a porter watches. 
Binder: 1918-1923 General Ads 3 of 10

Year: 1922

Slogan: Kodak as you Go

Subject(s): Man and woman

Text: none

Implied Audience: travelers

Product: Kodak

Photo or Illustration Based: photo

Color or Black and White: bw

Description / Notes: Photograph of a man and a woman

standing beside a car. The man holds the camera, the woman is pointing off in the distance.

Binder: 1918-1923 General Ads 3 of 10

Slogan: All Out-doors invites your Kodak

Subject(s): Man putting skiis on.

Text: none

Implied Audience: outdoors

Product: Kodak

Photo or Illustration Based: photo

Color or Black and White: bw

Description / Notes: Photograph of a man kneeling in the snow, putting on skis with Kodak slung over his shoulder.

Binder: $1918-1923$ General Ads 3 of 10

Slogan: All Out-doors invites your Kodak

Subject(s): Man XC Skiing

Text: none

Implied Audience: outdoorsmen

Product: Kodak

Photo or Illustration Based: photo

Color or Black and White: bw

Description / Notes: Photograph of a man cross-country skiing

away from the camera, with Kodak slung over his shoulder.

Binder: 1918-1923 General Ads 3 of 10

Slogan: For the out-of-doors days Kodak

Subject(s): man, two women
Text: And not merely the alluring picture story, but on every negative at least a date; and a title, too, if you like. Titling is the work of but an instant with an Autographic Kodak; is as simple as making the picture itself--and there is no extra charge for Autographic film.

Implied Audience: outdoors

Product: Kodak

Photo or Illustration Based: photo

Color or Black and White: bw

Description / Notes: Photograph of a woman with a Kodak, photographing a young couple in tennis outfits, holding racquets, and leaning on a net.

Binder: 1918-1923 General Ads 3 of 10

Slogan: Kodak as you Go

Subject(s): Men carrying a canoe

Text: Then you can go back at any time, without even leaving

your arm chair. The autographic feature tells you when and where each picture was taken.

Implied Audience: hunters / outdoorsman

Product: Kodak

Photo or Illustration Based: photo

Color or Black and White: bw

Description / Notes: Photograph of a group of men carrying a canoe through a forest. Caption reads: Kodak negative.

Binder: 1918-1923 General Ads 3 of 10

Slogan: Kodak as you Go

Subject(s): mother / child

Text: none

Implied Audience: families / travel

Product: Kodak

Photo or Illustration Based: photo

Color or Black and White: bw

Description / Notes: Photograph of a mother and daughter sitting in the back of a car, the girl holding the Kodak in her lap.

Binder: 1918-1923 General Ads 3 of 10

Year: 1920 
Slogan: The History of a Word

Subject(s): product - misc.

Product: Kodak Branding

Description / Notes: Trademark info

Binder: 1918-1923 General Ads 3 of 10

Year: 1920

Slogan: Two "k's", an "o", a "d" and an "a"

Subject(s): product - misc.

Product: Kodak Branding

Description / Notes: Trademark info

Binder: 1918-1923 General Ads 3 of 10

Year: 1920

Slogan: What "Kodak" Means

Subject(s): product - misc.

Product: Kodak Branding

Description / Notes: Trademark info

Binder: 1918-1923 General Ads 3 of 10

Slogan: Kodak Cartridges

Subject(s): Birds in a tree.

Text: Not game--but part of the lure of the wild just the same--

and worthy of your Kodak Cartridges.

Implied Audience: hunters / outdoorsman

Product: Kodak cartridges

Photo or Illustration Based: Photo

Color or Black and White: bw

Description / Notes: Photograph of seven birds on a tree.

Binder: 1918-1923 General Ads 3 of 10

Year: 1920

Slogan: Kodak Yourself and your catch

Subject(s): man

Text: A picture of your gamest fish taken in back of the house when you get home has not half the realism of the one you snap in front of the stream where you out-guessed your wily opponent hardly five minutes ago. Even if you go alone, you can get both your fish and yourself in the picture by taking along with your
Kodak these two unobtrusive little Kodak accessories..

Implied Audience: outdoors / men

Product: Kodak Self Timer, Kodapod

Photo or Illustration Based: photo-illustration

Color or Black and White: bw

Description / Notes: Photo of a man with a fish, posing for the camera. Overlayed arrows indicate the accessories.

Binder: 1918-1923 General Ads 3 of 10

Year: 1920

Slogan: Mile after mile of Eastman Film

Subject(s): product - film \& paper

Product: Motion Picture Film

Color or Black and White:

Binder: 1918-1923 General Ads 3 of 10

Year: 1920

Slogan: "Eastman" and "Kodak"

Subject(s): product - film \& paper

Product: Motion Picture Film

Color or Black and White:

Binder: $1918-1923$ General Ads 3 of 10

Year: 1920

Slogan: Eastman Film

Subject(s): product - film \& paper

Product: Motion Picture Film

Color or Black and White:

Binder: 1918-1923 General Ads 3 of 10

Slogan: There's a photographer in your town

Subject(s): none

Text: In a place of an Easter card--your photograph. Both

seasonable--but one a permanent reminder of you.

Implied Audience: Easter / general

Product: photograph

Photo or Illustration Based: Text: Color or Black and White: bw

Description / Notes: No photo - text only. 
Binder: 1918-1923 General Ads 4 of 10

Slogan: Make your Kodak Story Autographic

Subject(s): man in a canoe

Text: Keep on every negative the date and title, make your Kodak story not merely a picture story but also a record story; a story that will take you back to the year, the month, the very spot--will bring those outing days freshly before you even when time has played sad tricks with memory. Making the autographic record is the work of but a moment--and autographic films cost you no more then the other kind.

Implied Audience: outdoorsmen

Product: autographic Kodak

Photo or Illustration Based: photo

Color or Black and White: bw

Description / Notes: photograph of a man in the front of a

canoe, writing on the camera.

Binder: 1918-1923 General Ads 4 of 10

Slogan: Kodak on the Job

Subject(s): man on construction site.

Text: A daily negative, with its autographic date and title on the

film, makes the progress record authentic, indisputable.

Implied Audience: Laborers

Product: autographic Kodak

Photo or Illustration Based: photograph

Color or Black and White: bw

Description / Notes: photograph of a man at a construction site using the autographic Kodak.

Binder: 1918-1923 General Ads 4 of 10

Slogan: Make your Kodak Record authentic--autographic.

Subject(s): young man, woman

Text: Keep not merely the picture story of your travels but write the date and title on every film, at the time of exposure--before you forget. It's a matter of but a few seconds with an Autographic Kodak and autographic film. And the record is always there, on the edge of the film, as Permanent as the negative itself.

Implied Audience: trave
Product: autographic Kodak

Photo or Illustration Based: photo-illustration

Color or Black and White: bw

Description / Notes: Photograph of a young couple superimposed over a photo of a church, making it look as if they are standing outside a church.

Binder: 1918-1923 General Ads 4 of 10

Year: 1920

Slogan: "Kodak Literature"

Subject(s): product - misc.

Product: booklets

Description / Notes: List of available publications. "For the Amateur who wants to know the why of what he does."

Binder: 1918-1923 General Ads 4 of 10

Year: 1920

Slogan: Standardize your Exposure Technique with Eastman Dental X-Ray Films

Subject(s): product - medical

Product: Eastman Dental X-Ray Films

Color or Black and White

Binder: 1918-1923 General Ads 4 of 10

Year: 1920

Slogan: A Matter of Record--Eastman Dental X-Ray Films

Subject(s): product - medical

Product: Eastman Dental X-Ray Films

Color or Black and White:

Binder: 1918-1923 General Ads 4 of 10

Year: 1920

Slogan: "Angulation" Becomes extremely easy with Eastman

Dental X-Ray Films

Subject(s): product - medical

Product: Eastman Dental X-Ray Films

Description / Notes: Captions read: "Distorted" and "Correct"

Binder: $1918-1923$ General Ads 4 of 10 
Year: 1921

Slogan: Eastman Dental X-Ray Films Regular of Extra-Fast

Subject(s): product - medical

Product: Eastman Dental X-Ray Films

Color or Black and White:

Binder: 1918-1923 General Ads 4 of 10

Year: 1920

Slogan: Standardization.

Subject(s): product - medical

Product: Eastman Dupli-tized X-Ray Films

Color or Black and White:

Binder: 1918-1923 General Ads 4 of 10

Year: 1920

Slogan: For Adaptability--Eastman Dupli-Tized X-Ray Films.

Subject(s): product - medical

Product: Eastman Dupli-tized X-Ray Films

Color or Black and White:

Binder: 1918-1923 General Ads 4 of 10

Year: 1920

Slogan: Begin with Eastman Dupli-Tized X-Ray Films

Subject(s): product - medical

Product: Eastman Dupli-tized X-Ray Films

Color or Black and White:

Binder: 1918-1923 General Ads 4 of 10

Year: 1921

Slogan: For Use with the Modified Bucky Diaphragm. Eastman Dupli-Tized X-Ray Films

Subject(s): product - medical

Product: Eastman Dupli-tized X-Ray Films

Color or Black and White:

Binder: 1918-1923 General Ads 4 of 10

Year: 1920

Slogan: Eastman Organic Chemicals

Subject(s): product - Chemicals
Product: Eastman Organic Chemicals

Color or Black and White:

Binder: 1918-1923 General Ads 4 of 10

Year: 1920

Slogan: Eastman Organic Chemicals

Subject(s): product - Chemicals

Product: Eastman Organic Chemicals

Color or Black and White:

Binder: 1918-1923 General Ads 4 of 10

Year: 1920

Slogan: Standardize your dark-room technique with Eastman Prepared X-Ray Developer Powders

Subject(s): product - medical

Product: Eastman X-Ray Developing Powders

Color or Black and White:

Binder: 1918-1923 General Ads 4 of 10

Year: 1920

Slogan: Standardization in Radiographic Technique

Subject(s): product - medical

Product: Eastman X-Ray products

Color or Black and White:

Binder: 1918-1923 General Ads 4 of 10

Year: 1921

Slogan: The Eastman X-Ray Reducing Camera

Subject(s): product - medical

Product: Eastman X-Ray Reducing Camera

Color or Black and White:

Binder: 1918-1923 General Ads 4 of 10

Slogan: Let Kodak Sell your Livestock.

Subject(s): Cow

Text: You know cattle and horses and sheep and hogs. You know the pose that will bring out their strong points. The rest is easy.

Kodak has so simplified photography that anybody can make good pictures--and it's less expensive than you think. 
Implied Audience: farmers

Product: Kodak

Photo or Illustration Based: photo

Color or Black and White: bw

Description / Notes: Photograph of a cow - the caption reads: A

Holstein King in a Kingly pose

Binder: $1918-1923$ General Ads 4 of 10

Slogan: None

Subject(s): Fish

Text: The thrill of the strike, the swish of the line, the whirr of the

reel, the myriad diamonds flashing in the sun as King Speckle-

sides leaps from the pool in futile fury, the filled creel--Kodak can

keep for you all these and bring them back at will--At your will

Implied Audience: outdoorsmen

Product: Kodak

Photo or Illustration Based: photo

Color or Black and White: bw

Description / Notes: Photograph of huge dead fish on rocks,

with nets and fishing poles.

Binder: $1918-1923$ General Ads 4 of 10

Slogan: Kodak as you Go

Subject(s): Kodak Gir

Text: none

Implied Audience: women / general

Product: Kodak

Photo or Illustration Based: photo

Color or Black and White: bw

Description / Notes: Photograph of a well dressed woman with a Kodak climbing into the front seat of a car.

Binder: $1918-1923$ General Ads 4 of 10

Slogan: All Out-doors invites your Kodak

Subject(s): Man with two dogs.

Text: None

Implied Audience: outdoorsmen

Product: Kodak

Photo or Illustration Based: photo
Color or Black and White: bw

Description / Notes: Photograph of a man in a snowy field with two dogs, holding a gun. Caption reads: From a Kodak Negative.

Binder: 1918-1923 General Ads 4 of 10

Slogan: Kodak as you Go

Subject(s): man, woman

Text: none

Implied Audience: travel

Product: Kodak

Photo or Illustration Based: photo

Color or Black and White: bw

Description / Notes: Photograph of a young couple. The woman is skiing, the man is holding a Kodak, and a sled.

Binder: 1918-1923 General Ads 4 of 10

Slogan: There's a photographer in your town

Subject(s): none

Text: The Christmas gift with the personal touch-- your

photograph.

Implied Audience: Christmas

Product: Kodak

Photo or Illustration Based: Text: Color or Black and White: bw

Description / Notes: No photo - text only.

Binder: 1918-1923 General Ads 4 of 10

Slogan: There's Game for your Kodak, as well as game for your gun--and it's all apart of the lure of the wild.

Subject(s): photographs of animals

Text: none

Implied Audience: outdoorsmen

Product: Kodak

Photo or Illustration Based: photograph

Color or Black and White: bw

Description / Notes: Seven photographs of animals: raccoon, porcupine, owls, crane, baby birds.

Binder: 1918-1923 General Ads 4 of 10 
Slogan: Complete the Kodak Story--with the Autographic date and title.

Subject(s): two women and a man.

Text: none

Implied Audience: outdoors

Product: Kodak

Photo or Illustration Based: Text: Color or Black and White: bw

Description / Notes: Two women sit, one with a Kodak, as she writes on it. A man stands, holding a set of golf clubs.

Binder: $1918-1923$ General Ads 4 of 10

Slogan: Kodak

Subject(s): young girl

Text: Means most in the home, because home pictures tell the simple every day story of the children--each one a fascinating chapter for the Kodak Album.

Implied Audience: families

Product: Kodak

Photo or Illustration Based: photo

Color or Black and White: bw

Description / Notes: Photograph of a girl at a window. Close

cropped - shoulders and head only.

Binder: 1918-1923 General Ads 4 of 10

Slogan: none

Subject(s): young girl

Text: After all, pictures of the children, just every day pictures in and about the home are the ones we care for most. Such pictures are easily made with a Kodak or Brownie and the expense is less than you think.

Implied Audience: families

Product: Kodak

Photo or Illustration Based: photo

Color or Black and White: bw

Description / Notes: Portrait of a young girl in a hat standing

outside her house. Caption reads: From a 2a Brownie negative.

Binder: 1918-1923 General Ads 4 of 10
Slogan: Kodak

Subject(s): young girl

Text: Means most in the home, because home pictures tell the simple every day story of the children--each one a fascinating chapter for the Kodak Album.

Implied Audience: families

Product: Kodak

Photo or Illustration Based: photo

Color or Black and White: bw

Description / Notes: Same text as above ad, but with a

photograph of a different young girl, less closely cropped. She is sitting in a library, wearing a headband.

Binder: 1918-1923 General Ads 4 of 10

Slogan: When the children outgrow Childhood.

Subject(s): young girl.

Text: After all home pictures mean the most. Pictures of travel, of the week-end motor trip and of our sports--all these add to the fascination of the picture album. But the home pictures--for the most part pictures of the children, just everyday pictures--these are the ones that never lose their appeal, that grow more and more in value as the children out-grow childhood.

Implied Audience: families

Product: Kodak

Photo or Illustration Based: photo

Color or Black and White: bw

Description / Notes: Photograph of a young girl in a hat--same photograph as above but with different text.

Binder: 1918-1923 General Ads 4 of 10

Year: 1921

Slogan: Kodak means most in the home, because home pictures tell simple everyday stories of the children.

Subject(s): product - misc.

Product: Kodak Album

Color or Black and White:

Binder: 1918-1923 General Ads 4 of 10

Year: 1920 
Slogan: Among the Christmas Gifts

Subject(s): product - camera

Product: Kodak Cameras

Description / Notes: A list of the available Kodak Cameras, including $3 a$ Kodak, Vest Pocket Kodak, No. 1 Autographic Kodak Junior, and No. 0 Brownie

Binder: 1918-1923 General Ads 4 of 10

Slogan: There's a photographer in your town

Subject(s): none

Text: The boy and the girl. They are off to school; they will come back as grown ups--you wouldn't have it otherwise. But you will want always, a clear memory of them as they are to-day. Before they go--a photograph.

Implied Audience: families

Product: photograph

Photo or Illustration Based: Text: Color or Black and White: bw

Description / Notes: no photo- text only.

Binder: 1918-1923 General Ads 4 of 10

Slogan: There's a photographer in your town

Subject(s): none

Text: Not much chance for self-consciousness in the photographic portrait of to-day. Modern equipment enables the photographer to choose the happy moment and --it is done before you know it.

Product: photograph

Photo or Illustration Based: Text: Color or Black and White: bw

Description / Notes: No photo - text only.

Binder: 1918-1923 General Ads 4 of 10

Year: 1920

Slogan: You don't carry it; you wear it--like a watch. The Vest Pocket Kodak

Subject(s): product - camera

Product: Vest Pocket Kodak

Description / Notes: Price for camera: $\$ 9.49$. Film for 8 exposures: $\$ 0.25$.
Binder: $1918-1923$ General Ads 4 of 10

Year: 1920

Slogan: You don't carry a Vest Pocket Kodak; you wear it like a watch.

Subject(s): product - camera

Product: Vest Pocket Kodak

Description / Notes: Price for camera: \$9.49. Film for 8

exposures: $\$ 0.25$.

Binder: 1918-1923 General Ads 4 of 10

Year: 1921

Slogan: Service.

Subject(s): product - medical

Product: X-Ray Department at Rochester.

Color or Black and White:

Binder: 1918-1923 General Ads 5 of 10

Year: 1921

Slogan: Kodak Keeps the Record

Subject(s): construction workers

Text: And on every negative the indisputable autographic date

and title, made at the time.

Implied Audience: Laborers

Product: Autographic Kodak

Photo or Illustration Based: photo

Color or Black and White: bw

Description / Notes: photograph of a man with a Kodak, writing

on it, at a construction site, while two other men work.

Binder: $1918-1923$ General Ads 5 of 10

Year: 1921

Slogan: Kodak on the Job

Subject(s): construction workers

Text: There's a simple, certain way of keeping an authentic, indisputable record of the progress of every contract. With an Autographic Kodak you get not merely the picture, but all the important date and title on every negative.

Implied Audience: Laborers 
Product: autographic Kodak

Photo or Illustration Based: photo

Color or Black and White: bw

Description / Notes: Photograph of a man writing on a camera, while standing on a house construction site.

Binder: 1918-1923 General Ads 5 of 10

Year: 1921

Slogan: The Picture, the date, the title--you get them all with an Autographic Kodak.

Subject(s): Man, two women

Text: none

Implied Audience: travel

Product: autographic Kodak

Photo or Illustration Based: photo

Color or Black and White: bw

Description / Notes: Photograph of a man, two women out in the snow. The man is inscribing the picture, though the woman has a camera on her shoulder.

Binder: 1918-1923 General Ads 5 of 10

Year: 1921

Slogan: The Picture, the date, the title--you get them all with an Autographic Kodak.

Subject(s): Woman and two men.

Text: And when you are home again, making up your album

travelogue, there, on every negative, you can have the answer to each "Where and when was this taken?"

Implied Audience: travelers

Product: autographic Kodak

Photo or Illustration Based: photo-illustration

Color or Black and White: bw

Description / Notes: Photograph of a man and woman on

vacation, the woman holding the camera, the man pointing in the distance, while a man sits beside them. It is unclear where they are.

Binder: 1918-1923 General Ads 5 of 10

Year: Mar-21
Slogan: Kodak on the Job

Subject(s): product - camera

Product: Autographic Kodak

Color or Black and White:

Binder: 1918-1923 General Ads 5 of 10

Slogan: The Brownie Boy

Subject(s): young boy.

Text: None

Implied Audience: parents/children

Product: Brownie

Photo or Illustration Based: photo

Color or Black and White: bw

Description / Notes: Photograph of a young boy in a field posing for the camera. The Caption reads "No. 2 Brownie Negative."

Binder: 1918-1923 General Ads 5 of 10

Year: Apr-21

Slogan: For your Convenience Eastman Dental Film Safe.

Subject(s): product - medical

Product: Eastman Dental Film Safe

Description / Notes: Price: $\$ 3.50$

Binder: 1918-1923 General Ads 5 of 10

Year: Apr-21

Slogan: For Diagnosis and Record--the use of Eastman Dental XRay Films.

Subject(s): product - medical

Product: Eastman Dental X-Ray Films

Color or Black and White:

Binder: 1918-1923 General Ads 5 of 10

Year: May-21

Slogan: The Symbol of Quality on Each Packet of Eastman Dental X-Ray Films

Subject(s): product - medical

Product: Eastman Dental X-Ray Films

Color or Black and White: 
Binder: 1918-1923 General Ads 5 of 10

Year: 1921

Slogan: Eastman Dupli-Tized X-Ray Films

Subject(s): product - medical

Product: Eastman Dupli-tized X-Ray Films

Color or Black and White:

Binder: $1918-1923$ General Ads 5 of 10

Year: Mar-21

Slogan: For Safety. Eastman Dupli-Tized X-Ray Films

Subject(s): product - medical

Product: Eastman Dupli-tized X-Ray Films

Color or Black and White:

Binder: 1918-1923 General Ads 5 of 10

Year: Apr-21

Slogan: Film Efficiency A Fact--not an experiment.

Subject(s): product - medical

Product: Eastman Dupli-tized X-Ray Films

Color or Black and White:

Binder: 1918-1923 General Ads 5 of 10

Year: Apr-21

Slogan: Standardized Chest Technique

Subject(s): product - medical

Product: Eastman Dupli-tized X-Ray Films

Color or Black and White:

Binder: 1918-1923 General Ads 5 of 10

Year: 1921

Slogan: Load with the dependable film that comes in the Yellow Box--Eastman.

Subject(s): product - film \& paper

Product: Eastman N.C. and Autographic Film

Color or Black and White:

Binder: 1918-1923 General Ads 5 of 10

Year: 1921
Slogan: Eastman Organic Chemicals.

Subject(s): product - Chemicals

Product: Eastman Organic Chemicals

Color or Black and White:

Binder: 1918-1923 General Ads 5 of 10

Year: Mar-21

Slogan: Eastman Organic Chemicals

Subject(s): product - Chemicals

Product: Eastman Organic Chemicals

Description / Notes: "New price list with over 900 items"

Binder: 1918-1923 General Ads 5 of 10

Year: May-21

Slogan: The Manufacture of Eastman Organic Chemicals

Subject(s): product - Chemicals

Product: Eastman Organic Chemicals

Color or Black and White:

Binder: 1918-1923 General Ads 5 of 10

Year: May-21

Slogan: Eastman Organic Chemicals. During the past month the

following chemicals have become available:

Subject(s): product - Chemicals

Product: Eastman Organic Chemicals

Color or Black and White:

Binder: 1918-1923 General Ads 5 of 10

Year: 1921

Slogan: Graflex

Subject(s): product - camera

Product: Graflex Camera

Description / Notes: "Folmer \& Schwing Department"

Binder: 1918-1923 General Ads 5 of 10

Year: 1921

Slogan: Graflex. The Camera that does the difficult things in

photography--and does them well.

Subject(s): product - camera 
Product: Graflex Camera

Color or Black and White:

Binder: 1918-1923 General Ads 5 of 10

Year: 1921

Slogan: Graflex. The Choice of those sportsmen who know

cameras as they know guns and tackle.

Subject(s): product - camera

Product: Graflex Camera

Description / Notes: Caption reads: "From a Kodak Negative."

Binder: 1918-1923 General Ads 5 of 10

Year: May-21

Slogan: Graflex

Subject(s): product - camera

Product: Graflex Camera

Color or Black and White:

Binder: $1918-1923$ General Ads 5 of 10

Year: 1921

Slogan: Kodak

Subject(s): dog

Text: Every phase of out-door sport means more with a Kodak

along. Not merely your friends and your camp but your dogs, of course they are your friends too, the game that you shoot and the catch you make--all these add to the delights of your Kodak

album.

Implied Audience: hunters / outdoorsman

Product: Kodak

Photo or Illustration Based: photo

Color or Black and White: bw

Description / Notes: Photograph of a hunting dog -- caption reads "From a Kodak Negative"

Binder: 1918-1923 General Ads 5 of 10

Year: 1921

Slogan: Kodak as you Go

Subject(s): Kodak Girl

Text: None
Implied Audience: women

Product: Kodak

Photo or Illustration Based: photo

Color or Black and White: bw

Description / Notes: Photograph of a woman in a striped dress, standing in a field.

Binder: 1918-1923 General Ads 5 of 10

Slogan: The Great Out-doors Invites your Kodak

Subject(s): Man on horseback

Text: none

Implied Audience: outdoorsmen

Product: Kodak

Photo or Illustration Based: photo

Color or Black and White: bw

Description / Notes: photograph of a man on a horse, riding

away from the camera, through the forest. The camera isn't visible in the image.

Binder: 1918-1923 General Ads 5 of 10

Year: 1921

Slogan: There's always more fun with a Kodak along

Subject(s): teenage boy

Text: none

Implied Audience: parents/children

Product: Kodak

Photo or Illustration Based: Photo

Color or Black and White: bw

Description / Notes: Photograph of a boy with a fishing rod caption reads "From a Kodak Negative"

Binder: 1918-1923 General Ads 5 of 10

Year: 1921

Slogan: Keep a Kodak Story of the Children.

Subject(s): three young girls

Text: none

Implied Audience: families

Product: Kodak

Photo or Illustration Based: photo 


\section{Color or Black and White: bw}

Description / Notes: Photograph of three young girls outside.

The caption reads "Gossip."

Binder: 1918-1923 General Ads 5 of 10

Year: 1921

Slogan: none

Subject(s): two boys

Text: There's always more fun with a Kodak along. There's fun at the time in the picture making--and afterwards the pleasure for you and your friends in the Kodak album.

Implied Audience: parents/children

Product: Kodak

Photo or Illustration Based: photo

Color or Black and White: bw

Description / Notes: Photograph of two boys with a fishing pole and a fish, walking down a dirt road. Caption reads "From a Kodak Negative"

Binder: $1918-1923$ General Ads 5 of 10

Year: 1921

Slogan: Kodak on the Farm

Subject(s): two boys and a cow.

Text: On the farm Kodak serves a double purpose. Like the auto it is ready for business of for pleasure. It is always ready for pictures in and about the home. The children, the family pets, the grange picnic, the thousand and one familiar scenes around the farm--all these contribute delightfully to the family Kodak album. And there's a worth-while business use for the Kodak on every farm. It keeps the record of crop and orchard growths, the progress of building, the condition of the "line fence". It sells live stock.

Implied Audience: families / farmers

Product: Kodak

Photo or Illustration Based: photo

Color or Black and White: bw

Description / Notes: Photograph of two boys and a calf.

Binder: 1918-1923 General Ads 5 of 10

Year: 1921
Slogan: Keep a Kodak Story of the Children

Subject(s): two girls

Text: none

Implied Audience: families

Product: Kodak

Photo or Illustration Based: photo

Color or Black and White: bw

Description / Notes: Photograph of two young girls picking

wildflowers in a field. Caption reads "From a Kodak negative"

Binder: $1918-1923$ General Ads 5 of 10

Slogan: Keep a Kodak Story of the Children

Subject(s): young girl

Text: none

Implied Audience: families

Product: Kodak

Photo or Illustration Based: photo

Color or Black and White: bw

Description / Notes: Photograph of a young girl sitting on a park

bench, holding flowers. Caption reads "From a Kodak Negative"

Binder: $1918-1923$ General Ads 5 of 10

Slogan: Five-O'clock Tea

Subject(s): Young girl with doll

Text: Every day, in their little-world-of-make-believe, the children offer countless Kodak opportunities. Mary, entertaining at five-

o'clock tea, or with motherly solicitude putting Dolly to bed; little Jim manfully mastering his spirited steed or in Indian garb

carefully stalking a mountain lion, impersonated by Tabby who is blissfully blinking beneath hydrangeas--such are the pictures that mean the most. It is pictures of these every-day happenings that give to the Kodak Album its intimate, human interest. To-day it is full of charm; to-morrow, when the children have outgrown

childhood, it will be priceless.

Implied Audience: families

Product: Kodak

Photo or Illustration Based: photo

Color or Black and White: bw

Description / Notes: Photograph of a girl having a tea party with 
a doll.

Binder: 1918-1923 General Ads 5 of 10

Slogan: Keep a Kodak Story of the Children

Subject(s): young girl.

Text: Wherever children are, there pictures are, always. But to-

morrow it will be a different picture, for all too quickly the children out-grow childhood. Let there be no skips, no missing chapter in

the Kodak story of the children as told by your Kodak album.

Implied Audience: families

Product: Kodak

Photo or Illustration Based: photo

Color or Black and White: bw

Description / Notes: Photograph of a small girl, in white dress

and hat--the caption reads "1A Kodak Special."

Binder: $1918-1923$ General Ads 5 of 10

Slogan: All Out-doors invites your Kodak

Subject(s): Young man in a canoe

Text: none

Implied Audience: hunters / outdoorsman

Product: Kodak

Photo or Illustration Based: photo

Color or Black and White: bw

Description / Notes: Photograph of a young man standing in a

canoe, with a rifle, aiming it out onto a lake. Caption reads "From

a Kodak Negative."

Binder: 1918-1923 General Ads 5 of 10

Year: 1921

Slogan: A Kodak Self-Portrait

Subject(s): product - camera

Product: Kodak Portrait Attachment, Kodak Self-Timer

Description / Notes: Price for self-timer: \$1.25. Price for Portrait Attachment: $\$ 0.75$.

Binder: 1918-1923 General Ads 5 of 10

Year: 1921

Slogan: The Kodak and Close-ups
Subject(s): product - camera

Product: Kodak Portrait Attachment

Description / Notes: Price: $\$ 0.75$

Binder: 1918-1923 General Ads 5 of 10

Year: 1921

Slogan: The No. 0 Brownie. More Summer Fun and then--more winter fun

Subject(s): product - camera

Product: No. 0 Brownie

Description / Notes: Price: $\$ 2.00$

Binder: 1918-1923 General Ads 5 of 10

Year: May-21

Slogan: Enlarged from a negative made with a Two Dollar Brownie

Subject(s): product - camera

Product: No 0 Brownie

Color or Black and White:

Binder: $1918-1923$ General Ads 5 of 10

Year: Apr-21

Slogan: It is Kodak Made. The No. 2a Folding Brownie. $\$ 10.00$

Subject(s): product - camera

Product: No. 2a Folding Brownie.

Description / Notes: price: $\$ 10.00$

Binder: 1918-1923 General Ads 5 of 10

Year: Mar-21

Slogan: The No. 2c Autographic Kodak, Junior

Subject(s): product - camera

Product: No. 2c Autographic Kodak, Jr.

Description / Notes: Price: $\$ 25.00$

Binder: 1918-1923 General Ads 5 of 10

Year: May-21

Slogan: Vest Pocket Autographic Kodak, Special

Subject(s): product - camera

Product: Vest Pocket Autographic Kodak, Special.

Description / Notes: Price: $\$ 15.00$ 
Binder: 1918-1923 General Ads 5 of 10

Year: 1921

Slogan: Here is Efficiency. A Focusing Model of the Vest Pocket Kodak Special.

Subject(s): product - camera

Product: Vest Pocket Kodak Special.

Description / Notes: price: $\$ 21.00$

Binder: 1918-1923 General Ads 6 of 10

Year: 1922

Slogan: "At Home with the Kodak"

Subject(s): product - misc.

Product: "At Home with the Kodak" Booklet

Description / Notes: Free booklet

Binder: 1918-1923 General Ads 6 of 10

Year: 1922

Slogan: What the 2a Brownies do.

Subject(s): product - camera

Product: 2a Brownie

Description / Notes: Price: $\$ 3.50$

Binder: 1918-1923 General Ads 6 of 10

Year: 1921

Slogan: "There's no Room for Argument"

Subject(s): construction workers

Text: Every day, a Kodak negative of every job with an

autographic date and title and the progress record is indisputable.

Implied Audience: Laborers

Product: autographic Kodak

Photo or Illustration Based: photo

Color or Black and White: bw

Description / Notes: Photograph of a man writing on a camera, while standing on a house construction site.

Binder: 1918-1923 General Ads 6 of 10

Slogan: No Matter How Many Jobs

Subject(s): construction workers
Text: You can "keep tabs" on the daily progress of every contract with an Autographic Kodak. The date and title on the negative identify each picture definitely...

Implied Audience: Laborers

Product: autographic Kodak

Photo or Illustration Based: photo

Color or Black and White: bw

Description / Notes: photograph of a man at a construction site using the autographic Kodak.

Binder: 1918-1923 General Ads 6 of 10

Slogan: A Kodak history of the job.

Subject(s): construction workers

Text: Shows not only the progress made from day to day, but also the equipment used and methods employed. Such pictures prevent argument, because the autographic record tells the date, project, station, or similar information written on the film at the time of exposure. Thus the Kodak account of the job is Permanent and indisputable.

Implied Audience: Laborers

Product: autographic Kodak

Photo or Illustration Based: photo

Color or Black and White: bw

Description / Notes: Photograph of a man writing on a camera, standing in front of a digging machine.

Binder: 1918-1923 General Ads 6 of 10

Slogan: Kodak on the Job

Subject(s): workman

Text: It shows you how the job is progressing; its daily use serves to remind the workmen that you have a record of what they are accomplishing. The Autographic date and title, made in a moment, at the time the picture is taken makes the record indisputable.

Implied Audience: Laborers

Product: autographic Kodak

Photo or Illustration Based: photo

Color or Black and White: bw

Description / Notes: Photograph of a man writing on a camera standing in front of workers ant boats. 
Binder: 1918-1923 General Ads 6 of 10

Slogan: "It Checks up the Job"

Subject(s): workman

Text: Everyday a Kodak negative that shows what has been done, with an autographic date that is indisputable. The 3a Kodaks, for pictures of post-card size--a practical size for such records, from $\$ 19.00$ upwards.

Implied Audience: Laborers

Product: autographic Kodak

Photo or Illustration Based: photo

Color or Black and White: bw

Description / Notes: Photograph of a man standing near a train (?) car writing on his autographic Kodak.

Binder: 1918-1923 General Ads 6 of 10

Year: 1922

Slogan: For the Farm. No. 1 Autographic Kodak Junior. \$15.00

Subject(s): product - camera

Product: Autographic Kodak Junior.

Description / Notes: Price: $\$ 15.00$

Binder: 1918-1923 General Ads 6 of 10

Year: 1921

Slogan: A Page of Brownies

Subject(s): product - camera

Product: Brownie cameras

Description / Notes: Very large ad.

Binder: 1918-1923 General Ads 6 of 10

Year: 1922

Slogan: Announcing the Eastman Dental Film Mount

Subject(s): product - medical

Product: Eastman Dental Film Mount

Color or Black and White:

Binder: 1918-1923 General Ads 6 of 10

Year: 1922

Slogan: Announcing--Eastman Dental X-Ray Films Translucent
Base

Subject(s): product - medical

Product: Eastman Dental X-Ray Films

Color or Black and White:

Binder: 1918-1923 General Ads 6 of 10

Year: 1922

Slogan: Eastman Dupli-Tized X-Ray Films. Development, Fixing,

Washing and Drying.

Subject(s): product - medical

Product: Eastman Dupli-tized X-Ray Films

Color or Black and White:

Binder: 1918-1923 General Ads 6 of 10

Year: 1922

Slogan: Uniformity.

Subject(s): product - medical

Product: Eastman Dupli-tized X-Ray Films

Color or Black and White:

Binder: 1918-1923 General Ads 6 of 10

Year: 1922

Slogan: Eastman Organic Chemicals

Subject(s): product - Chemicals

Product: Eastman Organic Chemicals

Description / Notes: Graph.

Binder: 1918-1923 General Ads 6 of 10

Year: 1922

Slogan: Announcing-- Eastman Barium Sulphate for Roentgenology

Subject(s): product - Chemicals

Product: Eastman Organic Chemicals

Color or Black and White:

Binder: 1918-1923 General Ads 6 of 10

Year: 1922

Slogan: Announcing Eastman X-Ray Tank. Eastman X-Ray

Illuminator 
Subject(s): product - medical

Product: Eastman X-Ray Tank. Eastman X-Ray Illuminator

Description / Notes: Price for tank: \$15.00. Price for

Illuminator: $\$ 16.50$

Binder: 1918-1923 General Ads 6 of 10

Year: 1921

Slogan: Let your Kodak Keep the Christmas Story

Subject(s): Family

Text: none

Implied Audience: families

Product: Kodak

Photo or Illustration Based: photo

Color or Black and White: bw

Description / Notes: Photograph of a family -- mother and

father standing in the doorway, mother with camera,

photographing child asleep on grandfathers lap. Toys spread out on the floor.

Binder: 1918-1923 General Ads 6 of 10

Year: 1921

Slogan: Kodak

Subject(s): young girl

Text: An authentic, complete photographic record of livestock, farm equipment, crops --that's business. Pictures like the one reproduced above--and every home abounds in such

opportunities--that's pleasure. Kodak does double duty on the farm.

Implied Audience: families / farmers

Product: Kodak

Photo or Illustration Based: photo

Color or Black and White: bw

Description / Notes: Photograph of a young girl holding a

knitting needle, sitting on a step.

Binder: 1918-1923 General Ads 6 of 10

Year: 1921

Slogan: The No. 2c Autographic Kodak, Junior

Subject(s): product - camera
Product: No. 2c Autographic Kodak, Jr.

Description / Notes: Price: $\$ 25.00$. Very large ad.

Binder: 1918-1923 General Ads 6 of 10

Year: 1921

Slogan: Kodak Anastigmat f.7.7

Subject(s): product - camera

Product: No. 2c Autographic Kodak, Jr.

Description / Notes: Price: $\$ 25.00$

Binder: 1918-1923 General Ads 6 of 10

Slogan: There's a photographer in your town

Subject(s): none

Text: They will go away to school as children; they will return as young men and young women. You want them to--but you also want the childhood memories. Photographs of the children never grow up.

Implied Audience: families

Product: photograph

Photo or Illustration Based: Text: Color or Black and White: bw

Description / Notes: no photo- text only.

Binder: 1918-1923 General Ads 6 of 10

Year: 1922

Slogan: The Special Kodaks

Subject(s): product - camera

Product: Special Kodaks

Description / Notes: Price: $\$ 50.00$ and up.

Binder: 1918-1923 General Ads 6 of 10

Year: 1922

Slogan: Achievement.

Subject(s): product - camera

Product: The Kodak Anastigmats

Color or Black and White:

Binder: 1918-1923 General Ads 6 of 10

Year: 1922 
Slogan: A Significant Fact

Subject(s): product - camera

Product: The Kodak Anastigmats

Color or Black and White:

Binder: 1918-1923 General Ads 6 of 10

Year: 1921

Slogan: They are your pictures.

Subject(s): boy on a horse

Text: It is when the picture shows something that you are

interested in, that to you, it becomes worth while. You do not

perhaps care anything about taking pictures (though many people

think it great fun) but the pictures themselves you do care for

when they are of people or places or things that are yours or that

you are interested in. There's a personal, human touch in pictures of your home, your children, your broad meadows and your fine cattle. There's interest to you in pictures that tell the story of your auto trip, your camping party, or of the picnic where your children had such a good time. It is because such pictures have the personal appeal that, in millions of homes, the snap-shot album has become the most thought of book in all the house. Picture taking is very simple with a Kodak or Brownie, and less expensive than you think.

Implied Audience: families / farmers

Product: Photo or Illustration Based: photo

Color or Black and White: bw

Description / Notes: photograph of a boy in a pond riding a

horse, the horse is drinking from a pond.

Binder: 1918-1923 General Ads 7 of 10

Year: 1922

Slogan: "At Home with the Kodak"

Subject(s): product - misc.

Product: "At Home with the Kodak" Booklet

Description / Notes: Caption: "Made with Kodak and Kodak Portrait Attachment"

Binder: 1918-1923 General Ads 7 of 10

Year: Jan-22
Slogan: Keep the story of the children with an Autographic Kodak Subject(s): Child

Text: Pictures of the children gather interest with the years--and as the picture gains in interest, the date gains in importance.

"When was it made?" That's the inevitable question that a picture

of a child provokes. You know the answer now perhaps,--but later-

- Through the autographic feature, exclusively Eastman, and an

integral part of the Kodak, each negative may be dated--and titled

too,--at the time of exposure. The result is all the story--for all

time.

Implied Audience: families

Product: autographic Kodak

Photo or Illustration Based: Photo

Color or Black and White: bw

Description / Notes: Photograph of a young girl leaning on a

window sill, kneeling on a couch. The caption reads "From a Kodak Negative."

Binder: 1918-1923 General Ads 7 of 10

Slogan: Kodak Pictures of the Job

Subject(s): man on construction site.

Text: Construction methods, equipment used, progress of the work from day to day -- you can keep track of them easily with a

Kodak on the job. Daily negatives, each dated and titled at the time of exposure, make a progress record that is accurate and indisputable.

Implied Audience: Laborers

Product: autographic Kodak

Photo or Illustration Based: photo

Color or Black and White: bw

Description / Notes: Photograph of a man on a building site, writing on an autographic Kodak.

Binder: 1918-1923 General Ads 7 of 10

Year: 1922

Slogan: Winter Days Invite your Kodak

Subject(s): man, woman

Text: none

Implied Audience: outdoors / women 
Product: autographic Kodak

Photo or Illustration Based: photo

Color or Black and White: bw

Description / Notes: Photograph of a young man and woman standing in a doorway of a house. They are dressed for the

outdoors. Woman with a camera in her hand, man smoking a pipe.

Binder: 1918-1923 General Ads 7 of 10

Year: 1922

Slogan: Eastman Dental Film Developing Cabinet / The Tank Unit Subject(s): product - medical

Product: Eastman Dental Film Developing Cabinet / The Tank Unit Description / Notes: Price for developing cabinet and tank: $\$ 75.00$. Tank unit only: $\$ 25.00$.

Binder: 1918-1923 General Ads 7 of 10

Year: 1922

Slogan: Eastman Dental Film Safe

Subject(s): product - medical

Product: Eastman Dental Film Safe

Description / Notes: Price: $\$ 3.50$

Binder: 1918-1923 General Ads 7 of 10

Year: 1922

Slogan: A New Film in a New Package. Eastman Dupli-Tized X-Ray Films. Translucent Base.

Subject(s): product - medical

Product: Eastman Dupli-tized X-Ray Films

Color or Black and White:

Binder: 1918-1923 General Ads 7 of 10

Year: 1922

Slogan: Eastman Organic Chemicals bear labels with this seal which means purity and uniformity:

Subject(s): product - Chemicals

Product: Eastman Organic Chemicals

Color or Black and White:

Binder: 1918-1923 General Ads 7 of 10
Year: 1922

Slogan: Eastman Organic Chemicals

Subject(s): product - Chemicals

Product: Eastman Organic Chemicals

Description / Notes: "America's Greatest Stock. Nearly 1300

available"

Binder: 1918-1923 General Ads 7 of 10

Year: 1922

Slogan: Eastman Organic Chemicals

Subject(s): product - Chemicals

Product: Eastman Organic Chemicals

Description / Notes: Special Reagents

Binder: 1918-1923 General Ads 7 of 10

Year: 1922

Slogan: Eastman X-Ray Developer Powders Five Gallon Units

Subject(s): product - medical

Product: Eastman X-Ray Developing Powders

Color or Black and White:

Binder: 1918-1923 General Ads 7 of 10

Slogan: You will make good pictures with a Brownie

Subject(s): Dog

Text: The Folding Brownies particularly, offer just that

photographic equipment you want. They are such capable cameras and so easy to work that the pictures you make of your pets, your friends and your sports will be good pictures--from the start.

Implied Audience: parents/children

Product: Folding Brownie.

Photo or Illustration Based: photo

Color or Black and White: bw

Description / Notes: Photograph of a dog standing in a window.

The caption reads "From a Brownie Negative."

Binder: 1918-1923 General Ads 7 of 10

Slogan: Keep the Christmas Story with a Kodak

Subject(s): Child

Text: none 
Implied Audience: families

Product: Kodak

Photo or Illustration Based: photo

Color or Black and White: bw

Description / Notes: Same photo of young girl as above. Caption

reads "Jest 'fore Christmas I'm as good as I kin be." and "From a

Kodak Negative"

Binder: $1918-1923$ General Ads 7 of 10

Slogan: All Out-doors invites your Kodak

Subject(s): Kodak Girl

Text: none

Implied Audience: women / genera

Product: Kodak

Photo or Illustration Based: photo

Color or Black and White: bw

Description / Notes: Photograph of a woman on a beach with a

Kodak in her arms, waving at someone off camera.

Binder: $1918-1923$ General Ads 7 of 10

Year: Mar-22

Slogan: Let your Kodak catch the picture

Subject(s): Man and woman

Text: none

Implied Audience: outdoorsmen

Product: Kodak

Photo or Illustration Based: photo

Color or Black and White: bw

Description / Notes: Photograph of a man fishing, while a

woman stands on a nearby rock photographing him.

Binder: $1918-1923$ General Ads 7 of 10

Year: Jan-22

Slogan: Take a Kodak with You

Subject(s): man, woman

Text: none

Implied Audience: outdoors

Product: Kodak

Photo or Illustration Based: photo
Color or Black and White: bw

Description / Notes: Photograph of same man and woman as above, but standing outside the house, on the porch. Woman still has Kodak on her shoulder, man holds snowshoes under his arm.

Binder: 1918-1923 General Ads 7 of 10

Slogan: Keep the story with a Kodak

Subject(s): Mother, children, grandmother

Text: Today it's a picture of Grandmother reading to the children.

Tomorrow it may be Bobbie playing traffic policeman or Aunt Edna at the wheel of her new car or Brother Bill back from college for

the week-end or-- There's always another story waiting for your Kodak.

Implied Audience: families

Product: Kodak

Photo or Illustration Based: photo

Color or Black and White: bw

Description / Notes: Photograph of a mother with a camera photographing three children standing around grandmother in a chair.

Binder: 1918-1923 General Ads 7 of 10

Year: 1922

Slogan: A Drastic Cut. Kodak and Graflex Prices.

Subject(s): product - camera

Product: Kodak and Graflex Cameras

Description / Notes: Price list for all cameras. "Many models are back to pre-war prices"

Binder: 1918-1923 General Ads 7 of 10

Year: 1922

Slogan: Books that are never finished

Subject(s): product - camera

Product: The Kodak Anastigmats

Color or Black and White:

Binder: 1918-1923 General Ads 7 of 10

Year: 1922

Slogan: When Light Rings Flash their Warning 
Subject(s): product - camera

Product: The Kodak Anastigmats

Color or Black and White:

Binder: 1918-1923 General Ads 7 of 10

Year: 1922

Slogan: An Aristocracy of Labor

Subject(s): product - camera

Product: The Kodak Anastigmats

Color or Black and White:

Binder: 1918-1923 General Ads 7 of 10

Year: 1922

Slogan: Aiming at a Common Bulls-Eye

Subject(s): product - camera

Product: The Kodak Anastigmats

Color or Black and White:

Binder: 1918-1923 General Ads 7 of 10

Year: 1922

Slogan: Testing the Infinitesimal

Subject(s): product - camera

Product: The Kodak Anastigmats

Color or Black and White:

Binder: 1918-1923 General Ads 7 of 10

Year: 1922

Slogan: Lens Achievement

Subject(s): product - camera

Product: The Kodak Anastigmats

Color or Black and White:

Binder: 1918-1923 General Ads 8 of 10

Year: 1922

Slogan: Auto Graflex Junior

Subject(s): product - camera

Product: Auto Graflex Junior

Description / Notes: Price: $\$ 85.00$. small ad
Binder: 1918-1923 General Ads 8 of 10

Slogan: Let Kodak Sell your Livestock.

Subject(s): Cow

Text: You know how to pose your cattle, horses sheep or hogs so as to bring out their strong points and you can leave the rest to

the lens. Kodak pictures tell the story. Each print is the strongest kind of a selling argument. Making pictures the Kodak way is

inexpensive--and easy.

Implied Audience: farmers

Product: autographic Kodak

Photo or Illustration Based: photo

Color or Black and White: bw

Description / Notes: photo of a Cow in front of milk jugs.

Binder: 1918-1923 General Ads 8 of 10

Year: May / June 1922

Slogan: Take a Kodak with You

Subject(s): Father, daughter and son

Text: Dad showing Billie and Bess a trick of the trade makes a

picture you want of the fishing excursion. There will be plenty of such trips this summer--even if you only get away a day at a time-and chances for pictures are endless. The farm itself is full of

them. It's all easy the Kodak way.

Implied Audience: families

Product: autographic Kodak

Photo or Illustration Based: photo

Color or Black and White: bw

Description / Notes: Photograph of a father on the dock with his son and daughter.

Binder: $1918-1923$ General Ads 8 of 10

Year: 1922

Slogan: Eastman Dental X-Ray Films With Transparent Base

Subject(s): product - medical

Product: Eastman Dental X-Ray Films

Color or Black and White:

Binder: $1918-1923$ General Ads 8 of 10

Year: 1922 
Slogan: Eastman Dental X-Ray Films in the new package

Subject(s): product - medical

Product: Eastman Dental X-Ray Films

Color or Black and White:

Binder: 1918-1923 General Ads 8 of 10

Year: 1922

Slogan: The Standard Radiographic Medium. Eastman Dupli-Tized

X-Ray Film

Subject(s): product - medical

Product: Eastman Dupli-tized X-Ray Films

Color or Black and White:

Binder: 1918-1923 General Ads 8 of 10

Year: 1922

Slogan: Introducing Eastman Dupli-Tized X-Ray Films in 6-Dozen

Packages

Subject(s): product - medical

Product: Eastman Dupli-tized X-Ray Films

Color or Black and White:

Binder: $1918-1923$ General Ads 8 of 10

Year: 1922

Slogan: Uniformity. Eastman Dupli-Tized X-Ray Films

Subject(s): product - medica

Product: Eastman Dupli-tized X-Ray Films

Color or Black and White:

Binder: $1918-1923$ General Ads 8 of 10

Year: 1922

Slogan: Eastman Organic Chemicals. Made in USA

Subject(s): product - Chemicals

Product: Eastman Organic Chemicals

Description / Notes: Illustration of "National Distribution of a Month's Sales"

Binder: 1918-1923 General Ads 8 of 10

Year: 1922

Slogan: Eastman Organic Chemicals new quality specifications
Subject(s): product - Chemicals

Product: Eastman Organic Chemicals

Color or Black and White:

Binder: 1918-1923 General Ads 8 of 10

Year: 1922

Slogan: Eastman Organic Chemicals. Quality Unsurpassed

Subject(s): product - Chemicals

Product: Eastman Organic Chemicals

Color or Black and White

Binder: $1918-1923$ General Ads 8 of 10

Year: 1922

Slogan: Graflex

Subject(s): product - camera

Product: Graflex Camera

Description / Notes: caption: "From a Graflex Negative made with a Kodak Anastigmat Lens"

Binder: 1918-1923 General Ads 8 of 10

Year: 1922

Slogan: The Graflex Camera

Subject(s): product - camera

Product: Graflex Camera

Description / Notes: small ad

Binder: 1918-1923 General Ads 8 of 10

Year: 1922

Slogan: In the Hood of a Graflex

Subject(s): product - camera

Product: Graflex Camera

Description / Notes: small ad

Binder: 1918-1923 General Ads 8 of 10

Year: 1922

Slogan: Good Composition is easy with the Graflex

Subject(s): product - camera

Product: Graflex Camera

Description / Notes: small ad 
Binder: $1918-1923$ General Ads 8 of 10

Slogan: Every sport is more sport with a Kodak

Subject(s): children

Text: Your album filled with pictures of good times will be the

book you like the best. Whether it's riding or fishing, tramping or camping, Kodak as you go.

Implied Audience: parents/children

Product: Kodak

Photo or Illustration Based: photo

Color or Black and White: bw

Description / Notes: Photo of a boy photographing a girl in a

bicycle-car, on a trail.

Binder: $1918-1923$ General Ads 8 of 10

Year: Jun-22

Slogan: Take a Kodak with You

Subject(s): mother / child with dog.

Text: It's all so easy the Kodak way, and the pictures, precious at

the time, will be priceless to you later. You can spend your

vacation and have it, too--in pictures.

Implied Audience: families / travel

Product: Kodak

Photo or Illustration Based: photo

Color or Black and White: bw

Description / Notes: Photograph of a mother on the beach

photographing child hugging a sheepdog.

Binder: $1918-1923$ General Ads 8 of 10

Year: Jun-22

Slogan: Take a Kodak with You

Subject(s): two women and a man.

Text: Kodak film in the yellow box, over the counter all over the world.

Implied Audience: travelers

Product: Kodak

Photo or Illustration Based: photo

Color or Black and White: bw

Description / Notes: Photograph of two women and a man standing on a ship, sailing out of harbor. Man and one woman carry Kodaks.

Binder: $1918-1923$ General Ads 8 of 10

Year: 1922

Slogan: Your Dealer will show you how easy it is to make pictures like these with a Kodak

Subject(s): product - camera

Product: Kodak Cameras

Description / Notes: very large ad

Binder: $1918-1923$ General Ads 8 of 10

Year: 1922

Slogan: Your Kodak in Europe

Subject(s): product - film \& paper

Product: Kodak Film

Color or Black and White: Publications: The Ocean Times

Description / Notes: list of Kodak dealers in Europe

Binder: 1918-1923 General Ads 8 of 10

Year: May / June 1922

Slogan: Scout with a Kodak

Subject(s): Boy scout

Text: And when the hike carries you where dull light demands a time exposure--the woods, for example--there's the KODAPOD, a handy little Kodak convenience no bigger than your fist that

converts a fence rail, tree, or the handle of your scout hatchet into a tripod.

Implied Audience: parents/children/outdoors

Product: Kodapod

Photo or Illustration Based: photo

Color or Black and White: bw

Description / Notes: Photograph of a scout leaning on a tree

with his camera attached to the hatchet with a Kodapod.

Binder: 1918-1923 General Ads 8 of 10

Slogan: There's a photographer in your town

Subject(s): none

Text: At graduation time your friends expect your photograph 
Product: photograph

Photo or Illustration Based: Text: Color or Black and White: bw

Description / Notes: No photo - text only.

Binder: 1918-1923 General Ads 8 of 10

Year: 1922

Slogan: Offer the Premo

Subject(s): product - camera

Product: Premo Camera

Description / Notes: sales

Binder: $1918-1923$ General Ads 8 of 10

Year: 1922

Slogan: Your Premium Campaign will Succeed if you offer Premo Cameras

Subject(s): product - camera

Product: Premo Camera

Description / Notes: sales

Binder: 1918-1923 General Ads 8 of 10

Year: 1922

Slogan: A Premium that people want

Subject(s): product - camera

Product: Premo Camera

Color or Black and White:

Binder: $1918-1923$ General Ads 8 of 10

Year: 1922

Slogan: The Special Kodaks

Subject(s): product - camera

Product: Special Kodaks

Color or Black and White:

Binder: $1918-1923$ General Ads 8 of 10

Year: 1922

Slogan: An Eastman Product--The Premo Camera

Subject(s): product - camera

Product: The Premo Camera

\section{Description / Notes: sales}

Binder: $1918-1923$ General Ads 8 of 10

Year: 1922

Slogan: The Premo Camera is Known

Subject(s): product - camera

Product: The Premo Camera

Description / Notes: sales

Binder: 1918-1923 General Ads 9 of 10

Year: Mar-22

Slogan: The Hoyle of Amateur Photography

Subject(s): product - misc.

Product: "How to Make Good Pictures" Booklet

Description / Notes: Price: $\$ 0.40$

Binder: 1918-1923 General Ads 9 of 10

Year: Mar-22

Slogan: Depth is the quality which distinguishes the rich tone of

the print on Artura

Subject(s): product - film \& paper

Product: Artura Paper

Color or Black and White:

Binder: $1918-1923$ General Ads 9 of 10

Year: Apr-22

Slogan: Artura. The paper without a disappointment

Subject(s): product - film \& paper

Product: Artura Paper

Color or Black and White

Binder: 1918-1923 General Ads 9 of 10

Year: Jul-22

Slogan: Autographic Brownies

Subject(s): product - camera

Product: Autographic Brownies

Description / Notes: Price: From $\$ 9.00$ to $\$ 17.00$.

Binder: 1918-1923 General Ads 9 of 10 
Year: 22-Jul

Slogan: Kodak on the Farm

Subject(s): boy

Text: Kodak does double duty on the farm. Pictures like the one reproduced above--that's pleasure. A photographic record

complete even to the date and title of crops, stock, buildings and equipment--that's business. In either capacity it serves you well.

Implied Audience: farmers

Product: autographic Kodak

Photo or Illustration Based: photo

Color or Black and White: bw

Description / Notes: photograph of a boy in a haystack drinking milk.

Binder: $1918-1923$ General Ads 9 of 10

Slogan: Let Kodak tell the Story

Subject(s): Family

Text: This is a big day for Ed, Junior. To be allowed to go hunting with dad is a real event--and calls for a picture as a matter of course. Little story-telling incidents like this make the best kind of Kodak pictures. They are happening every day at your house.

Implied Audience: families

Product: autographic Kodak

Photo or Illustration Based: photo

Color or Black and White: bw

Description / Notes: Photo of father and son standing in the

yard, in hunting gear. Father has a gun, the boy has a Kodak on

his shoulder. The mother and daughter stand on the porch, mother taking a photo of the men leaving.

Binder: $1918-1923$ General Ads 9 of 10

Year: November / December 1922

Slogan: Keep Christmas With a Kodak

Subject(s): mother / daughter

Text: While far too excited to dress, little Jane has popped into bed again to pose for a picture with mother's new Kodak. And that only starts the fun. Even now father and Uncle Stan are renewing their youth in a snowball fight--and there's another picture. Kodak is a gift that slips out of the holiday box into the spirit of
Christmas.

Implied Audience: families / Christmas

Product: autographic Kodak

Photo or Illustration Based: photo

Color or Black and White: bw

Description / Notes: Photo of a mother photographing her daughter in bed.

Binder: 1918-1923 General Ads 9 of 10

Year: 1922

Slogan: Keep a Kodak Story of the Children

Subject(s): Mother, child and dog.

Text: none

Implied Audience: families

Product: autographic Kodak

Photo or Illustration Based: photo

Color or Black and White: bw

Description / Notes: photograph of a mother standing in the front yard photographing her daughter holding a dog.

Binder: 1918-1923 General Ads 9 of 10

Year: Aug-22

Slogan: There's a story at your house that Kodak can tell

Subject(s): Three children, and mother

Text: Whether or not the leading characters are the children, it is enough that the pictures are made at your house. Just this little fact gives them, as far as you and yours are concerned, an interest eternal. It's all easy the Kodak way. Pleasure from the start--and good pictures.

Implied Audience: families

Product: autographic Kodak

Photo or Illustration Based: photo

Color or Black and White: bw

Description / Notes: Same photo of boys with hose as above but with different text geared towards parents instead of children.

Binder: 1918-1923 General Ads 9 of 10

Year: 1922

Slogan: Kodak as you go 
Subject(s): Two boys, man and woman in car.

Text: The lens sees with you--the autographic record remembers for you-- and the story is complete for all time. Kodak brings back the trip to your library table.

Implied Audience: trave

Product: autographic Kodak

Photo or Illustration Based: photo

Color or Black and White: bw

Description / Notes: Photo of a young couple in the car, the woman is photographing two small boys on the road, one with a dead rabbit and a gun.

Binder: 1918-1923 General Ads 9 of 10

Slogan: Kodak brings your vacation back

Subject(s): two women

Text: All the fun you've had--all the friends you've made--your

vacation story told in split seconds, with a Kodak.

Implied Audience: women / travelers

Product: autographic Kodak

Photo or Illustration Based: photo

Color or Black and White: bw

Description / Notes: photo of a two woman, one leaning on a

tree on a beach, the other photographing her.

Binder: 1918-1923 General Ads 9 of 10

Year: Oct-22

Slogan: Pictures of your pets with a Brownie

Subject(s): Boy and dog

Text: With this simply-operated, Eastman made camera, it's good fun and good pictures from the start.

Implied Audience: parents/children

Product: Brownie

Photo or Illustration Based: photo

Color or Black and White: bw

Description / Notes: photo of a boy holding a folding Brownie in the yard, getting his dog to stand.

Binder: 1918-1923 General Ads 9 of 10

Year: Nov-22
Slogan: Bring Along A Brownie

Subject(s): three boys

Text: "Got those turkey pictures yet, Bill?" "Let's see the prints you made at the lake Saturday." "There's the picture you made of me and Doc?" Everybody likes pictures, and the Eastman-made, easily-worked Brownie gives you the kind you'll be glad to show.

Implied Audience: parents/children

Product: Brownie

Photo or Illustration Based: photo

Color or Black and White: bw

Description / Notes: Photo of three boys, two posing with a

turkey, one photographing.

Binder: $1918-1923$ General Ads 9 of 10

Year: Apr-22

Slogan: For Your Vest Pocket Kodak

Subject(s): product - camera

Product: Cable release attachment / Belt Carrying Case.

Description / Notes: Price for Cable release: $\$ 0.50$. Price for

carrying case: $\$ 1.50$ leather. $\$ 0.75$ leather.

Binder: $1918-1923$ General Ads 9 of 10

Year: Apr-22

Slogan: For Enlarging--Eastman Bromide Papers

Subject(s): product - film \& paper

Product: Eastman Bromide Papers

Color or Black and White

Binder: 1918-1923 General Ads 9 of 10

Year: May-22

Slogan: For Contact Prints use velox; for enlargements--Eastman Bromide Papers.

Subject(s): product - film \& paper

Product: Eastman Bromide Papers

Color or Black and White:

Binder: 1918-1923 General Ads 9 of 10

Year: 1922

Slogan: Announcing the Eastman Clinical Camera Outfit 
Subject(s): product - medical

Product: Eastman Clinical Camera Outfit

Description / Notes: Price: $\$ 160.00$. Includes Camera, stand, lights

Binder: 1918-1923 General Ads 9 of 10

Year: 1922

Slogan: Standardized Time-Temperature Development With

Eastman Dental X-Ray Films

Subject(s): product - medica

Product: Eastman Dental X-Ray Films

Color or Black and White:

Binder: 1918-1923 General Ads 9 of 10

Year: 1922

Slogan: Eastman Dental X-Ray Films Translucent or clear base

Subject(s): product - medica

Product: Eastman Dental X-Ray Films

Color or Black and White:

Binder: 1918-1923 General Ads 9 of 10

Year: Aug-22

Slogan: Standardized Time-Temperature Development With

Eastman Dupli-Tized X-Ray Films

Subject(s): product - medical

Product: Eastman Dupli-tized X-Ray Films

Color or Black and White:

Binder: 1918-1923 General Ads 9 of 10

Year: 1922

Slogan: Eastman Dupli-Tized X-Ray Films - In the Monogrammed Box.

Subject(s): product - medical

Product: Eastman Dupli-tized X-Ray Films

Color or Black and White:

Binder: 1918-1923 General Ads 9 of 10

Year: Apr-22

Slogan: Eastman Film Negative Album / Kodak Photo Paste
Subject(s): product - misc.

Product: Eastman Film Negative Album / Kodak Photo Paste

Description / Notes: price for Album: $\$ 0.75$ and up. Price for Photo Paste: $\$ 0.15$ per $20 z$ tube.

Binder: $1918-1923$ General Ads 9 of 10

Year: Jun-22

Slogan: Look for this seal on every package or bottle.

Subject(s): product - Chemicals

Product: Eastman Kodak Company Tested Chemicals

Color or Black and White:

Binder: 1918-1923 General Ads 9 of 10

Year: Jul-22

Slogan: Eastman Organic Chemicals

Subject(s): product - Chemicals

Product: Eastman Organic Chemicals

Color or Black and White:

Binder: 1918-1923 General Ads 9 of 10

Year: 1922

Slogan: Eastman Organic Chemicals

Subject(s): product - Chemicals

Product: Eastman Organic Chemicals

Description / Notes: Caption: "Apparatus for Hydrolysis of

Esters"

Binder: 1918-1923 General Ads 9 of 10

Year: 1922

Slogan: Eastman Organic Chemicals - Three Points

Subject(s): product - Chemicals

Product: Eastman Organic Chemicals

Color or Black and White:

Binder: 1918-1923 General Ads 9 of 10

Year: 1922

Slogan: Eastman Organic Chemicals

Subject(s): product - Chemicals

Product: Eastman Organic Chemicals 
Description / Notes: "Send for our September Price List"

Binder: 1918-1923 General Ads 9 of 10

Year: 1922

Slogan: Eastman Organic Chemicals - Widest Choice of Indicators

Subject(s): product - Chemicals

Product: Eastman Organic Chemicals

Color or Black and White:

Binder: 1918-1923 General Ads 9 of 10

Year: 1922

Slogan: Eastman Organic Chemicals - America's Largest Stock

Subject(s): product - Chemicals

Product: Eastman Organic Chemicals

Color or Black and White:

Binder: 1918-1923 General Ads 9 of 10

Year: Mar-22

Slogan: Eastman Portrait Bromide

Subject(s): product - film \& paper

Product: Eastman Portrait Bromide

Color or Black and White:

Binder: 1918-1923 General Ads 9 of 10

Year: Apr-22

Slogan: Make your large prints on Eastman Portrait Bromide

Subject(s): product - film \& paper

Product: Eastman Portrait Bromide

Color or Black and White:

Binder: 1918-1923 General Ads 9 of 10

Year: Jun-22

Slogan: The print on Eastman Portrait Bromide

Subject(s): product - film \& paper

Product: Eastman Portrait Bromide

Color or Black and White:

Binder: $1918-1923$ General Ads 9 of 10

Year: Apr-22
Slogan: Eastman Portrait Diffusion Disks

Subject(s): product - misc.

Product: Eastman Portrait Diffusion Disks

Description / Notes: Price: from $\$ 6.00$ to $\$ 7.50$

Binder: 1918-1923 General Ads 9 of 10

Year: Apr-22

Slogan: Eastman Portrait Film Par Speed--Super Speed

Subject(s): product - film \& paper

Product: Eastman Portrait Film

Color or Black and White:

Binder: 1918-1923 General Ads 9 of 10

Year: Mar-22

Slogan: What they think of the Eastman Projection Printer in England

Subject(s): product - misc.

Product: Eastman Projection Printer

Description / Notes: Letter from Reflex Studios in England.

Binder: 1918-1923 General Ads 9 of 10

Year: Apr-22

Slogan: Eastman Projection Printers

Subject(s): product - darkroom

Product: Eastman Projection Printer

Description / Notes: Price: for $4 \times 5$ negatives: $\$ 200.00$. For $5 \times 7$

negatives: $\$ 450.00$. For $8 \times 10$ Negatives: $\$ 675.00$

Binder: 1918-1923 General Ads 9 of 10

Year: May-22

Slogan: To Weigh easily--to measure easily and correctly

Subject(s): product - misc.

Product: Eastman Studio Scales / Eastman Visible Graduates

Description / Notes: Price for scale: \$4.50. Price for Graduates: $\$ 0.30$ to $\$ 1.00$

Binder: 1918-1923 General Ads 9 of 10

Year: Mar-22

Slogan: Making out Next Year's Income Tax Report Will Be Easy if 
You Now Install The Eastman Studio System

Subject(s): product - misc.

Product: Eastman Studio System

Color or Black and White:

Binder: 1918-1923 General Ads 9 of 10

Year: Jun-22

Slogan: Eastman View Cameras No.2

Subject(s): product - camera

Product: Eastman View Camera No. 2

Description / Notes: Price: from $\$ 42.00$ to $\$ 50.75$

Binder: 1918-1923 General Ads 9 of 10

Year: Jun-22

Slogan: Accessories that assist in making developing easy--and correct.

Subject(s): product - darkroom

Product: Eastman Visible Graduate / Eastman Thermometer

Description / Notes: price for graduate: $\$ 0.30$ and up. Price for

Thermometer: $\$ 0.75$

Binder: 1918-1923 General Ads 9 of 10

Year: 1922

Slogan: Introducing Eastman X-Ray Fixing Powders

Subject(s): product - medical

Product: Eastman X-Ray Fixing Powders.

Color or Black and White:

Binder: $1918-1923$ General Ads 9 of 10

Year: Mar-22

Slogan: Elon

Subject(s): product - Chemicals

Product: Elon Developer

Color or Black and White:

Binder: 1918-1923 General Ads 9 of 10

Year: Apr-22

Slogan: Elon. We Make it--We know it's right.

Subject(s): product - Chemicals
Product: Elon Developer

Color or Black and White:

Binder: 1918-1923 General Ads 9 of 10

Year: Jun-22

Slogan: When you Specify Elon

Subject(s): product - Chemicals

Product: Elon Developer

Color or Black and White:

Binder: 1918-1923 General Ads 9 of 10

Year: Aug-22

Slogan: There's always more fun with a Kodak

Subject(s): Three children, and mother

Text: First the fun of "shooting" the scene--and after that the pictures. Think of the things you would like to photograph--

incidents around the home like the one shown above, for example,

pictures of your friends, your sports, your trips, your pets. And it's

all easy with a Kodak or Brownie, as you can see for yourself when you look them over at the store.

Implied Audience: parents/children

Product: Kodak / brownie

Photo or Illustration Based: photo

Color or Black and White: bw

Description / Notes: Photo of three boys playing outside with a

hose while the mother watches and takes photos.

Binder: 1918-1923 General Ads 9 of 10

Year: May-22

Slogan: Kodak Acid Fixing Powder

Subject(s): product - Chemicals

Product: Kodak Acid Fixing Powder

Color or Black and White:

Binder: 1918-1923 General Ads 9 of 10

Year: Mar-22

Slogan: A New Enlarger--Kodak Auto-Focus Enlarger

Subject(s): product - darkroom

Product: Kodak Auto-Focus Enlarger 
Description / Notes: Price: $\$ 35.00$

Binder: 1918-1923 General Ads 9 of 10

Year: Jun-22

Slogan: Kodak Auto-Focus Enlarger

Subject(s): product - darkroom

Product: Kodak Auto-Focus Enlarger

Description / Notes: Price: $\$ 35.00$

Binder: $1918-1923$ General Ads 9 of 10

Year: Jul-22

Slogan: For Amateurs, the new Kodak Auto-Focus Enlarger

Subject(s): product - darkroom

Product: Kodak Auto-Focus Enlarger

Description / Notes: Price: $\$ 35.00$

Binder: 1918-1923 General Ads 9 of 10

Year: Jun-22

Slogan: Kodak Color Filter / Kodak Sky Filter

Subject(s): product - camera

Product: Kodak Color Filter / Kodak Sky Filter

Description / Notes: Price: $\$ 0.75$ to $\$ 1.50$

Binder: 1918-1923 General Ads 9 of 10

Year: Jul-22

Slogan: Announcing Kodak Cut Film

Subject(s): product - film \& paper

Product: Kodak cut Film

Description / Notes: Price from $\$ 0.46$ a dozen.

Binder: 1918-1923 General Ads 9 of 10

Year: Mar-22

Slogan: The Kodak Dry Mounting Press

Subject(s): product - misc.

Product: Kodak Dry Mounting Press

Color or Black and White:

Binder: 1918-1923 General Ads 9 of 10

Year: May-22
Slogan: Kodak Film Tank

Subject(s): product - darkroom

Product: Kodak Film Tank

Description / Notes: Price: $\$ 3.00$ and up

Binder: 1918-1923 General Ads 9 of 10

Year: Jul-22

Slogan: Finish the fun--develop your own vacation films with the

Kodak Film Tank

Subject(s): product - darkroom

Product: Kodak Film Tank

Description / Notes: Price: $\$ 3.00$ and up.

Binder: 1918-1923 General Ads 9 of 10

Year: Jun-22

Slogan: All-metal--Always Accurate. Eastman Metal Trimmer

Subject(s): product - misc.

Product: Kodak Metal Trimmer

Description / Notes: price: $\$ 15.00$

Binder: 1918-1923 General Ads 9 of 10

Year: Jun-22

Slogan: Kodak Metal Tripods

Subject(s): product - misc.

Product: Kodak Metal Tripods

Description / Notes: price: $\$ 2.75$ and up.

Binder: 1918-1923 General Ads 9 of 10

Year: Mar-22

Slogan: They help you make good pictures--

Subject(s): product - misc.

Product: Kodak Metal Tripods / Optipod

Description / Notes: price for metal tripod: $\$ 2.75$ and up. Price for Optipod: $\$ 1.25$

Binder: 1918-1923 General Ads 9 of 10

Year: Mar-22

Slogan: V.P.K. Tripod Adapter / Brownie Tripod Adapter

Subject(s): product - misc. 
Product: Kodak Tripod Adapters

Description / Notes: Price for V.P.K. adapter: $\$ 0.50$. Price for

Brownie Adapter: $\$ 0.50$

Binder: 1918-1923 General Ads 9 of 10

Year: Jun-22

Slogan: The Green of the Scene is in the tint of the print on Kodak Velvet Green Paper.

Subject(s): product - film \& paper

Product: Kodak Velvet Green Paper

Color or Black and White:

Binder: 1918-1923 General Ads 9 of 10

Year: 1922

Slogan: For Fun on the Farm and for practical pictures of the business of farming

Subject(s): product - camera

Product: No. 1 Autographic Kodak Junior

Description / Notes: Price: $\$ 12.00$

Binder: 1918-1923 General Ads 9 of 10

Year: Jul-22

Slogan: A new model Kodak

Subject(s): product - camera

Product: No. 1 Autographic Kodak Special

Color or Black and White:

Binder: 1918-1923 General Ads 9 of 10

Year: 1922

Slogan: The New No. 1 Autographic Kodak Special

Subject(s): product - camera

Product: No. 1 Autographic Kodak Special

Description / Notes: Price: $\$ 50.00$

Binder: 1918-1923 General Ads 9 of 10

Year: 1922

Slogan: No. 1 Autographic Kodak Special

Subject(s): product - camera

Product: No. 1 Autographic Kodak Special

\section{Description / Notes: Price: $\$ 50.00$}

Binder: 1918-1923 General Ads 9 of 10

Year: 1922

Slogan: Well Known and Well Liked--Premo Cameras

Subject(s): product - camera

Product: Premo Cameras

Color or Black and White:

Binder: 1918-1923 General Ads 9 of 10

Year: 1922

Slogan: Premiums that Produce. Premo Cameras

Subject(s): product - camera

Product: Premo Cameras

Description / Notes: Sales

Binder: 1918-1923 General Ads 9 of 10

Year: May-22

Slogan: To Record the Scenes of the Motor trip - The Panoram Kodak

Subject(s): product - camera

Product: The Panoram Kodak

Description / Notes: Price for No. 1: \$20.00. Price for No. 2. $\$ 30.00$

Binder: $1918-1923$ General Ads 9 of 10

Year: Mar-22

Slogan: For The Results you want, print on Velox

Subject(s): product - film \& paper

Product: Velox Paper

Color or Black and White

Binder: 1918-1923 General Ads 9 of 10

Year: Mar-22

Slogan: Ask your finisher to print your negatives on Velox

Subject(s): product - film \& paper

Product: Velox Paper

Color or Black and White: 
Binder: 1918-1923 General Ads 9 of 10

Year: Apr-22

Slogan: The Versatile Paper--Velox

Subject(s): product - film \& paper

Product: Velox Paper

Color or Black and White:

Binder: 1918-1923 General Ads 9 of 10

Year: May-22

Slogan: For Results from start to finish: Kodak, Kodak Film and Velox

Subject(s): product - film \& paper

Product: Velox Paper

Color or Black and White:

Binder: 1918-1923 General Ads 9 of 10

Year: Jul-22

Slogan: You can get the best print on Velox

Subject(s): product - film \& paper

Product: Velox Paper

Color or Black and White:

Binder: 1918-1923 General Ads 9 of 10

Year: Apr-22

Slogan: Velox Transparent Water Color Stamp Outfit

Subject(s): product - misc.

Product: Velox Transparent Water Color Stamps

Description / Notes: Price for the outfit: $\$ 1.00$

Binder: 1923-1925 General Ads \#1

Year: 1975

Slogan: Subject(s): Product: Advertisement

Description / Notes: Letter from Mary Hackett to Mr. Sweeting regarding a Kodak ad that used to be in this binder. Mr. Sweeting had wanted a copy of an ad, and Hackett sent the original instead.

Binder: 1923-1925 General Ads \#1

Slogan: Kodak keeps the story

Subject(s): Kodak Girl
Text: All the story--not only the picture but the date and title written on the film at the time. And this feature is exclusively Eastman.

Implied Audience: women / general

Product: autographic Kodak

Photo or Illustration Based: photo

Color or Black and White: bw

Description / Notes: Photograph of a woman in a checkered dress and hat holding a camera. The caption reads "Writing the Autographic Record."

Binder: 1923-1925 General Ads \#1

Slogan: All Out-doors invites your Kodak

Subject(s): Kodak Girl

Text: none

Implied Audience: women / travelers

Product: autographic Kodak

Photo or Illustration Based: photo

Color or Black and White: bw

Publications: The American Magazine

Description / Notes: Photograph of a woman on a fence in a striped dress holding a Kodak

Binder: 1923-1925 General Ads \#1

Slogan: Take a Kodak with you

Subject(s): man

Text: none

Implied Audience: travelers

Product: autographic Kodak

Photo or Illustration Based: photo

Color or Black and White: bw

Description / Notes: photograph of a man, chest down, holding

a Kodak, and his watch, with suitcases, and golf clubs at his feet.

Binder: 1923-1925 General Ads \#1

Slogan: Kodak is the ally of every other sport

Subject(s): Man on a mountain

Text: none

Implied Audience: outdoorsmen 
Product: autographic Kodak

Photo or Illustration Based: photo

Color or Black and White: bw

Description / Notes: Photo of a man sitting on top of a cliff with his Kodak.

Binder: 1923-1925 General Ads \#1

Year: May-23

Slogan: Keep a Kodak Story of the Children

Subject(s): mother/children

Text: none

Implied Audience: families

Product: autographic Kodak

Photo or Illustration Based: photo

Color or Black and White: bw

Description / Notes: Photo of a mother photographing three

children, one on a swing.

Binder: 1923-1925 General Ads \#1

Slogan: Kodak and Afterwards it's all in the album

Subject(s): three women and one man.

Text: none

Implied Audience: trave

Product: Autographic Kodak

Photo or Illustration Based: photo

Color or Black and White: bw

Description / Notes: Photo of a woman at the side of the water, holding a Kodak, photographing two woman and a man swimming.

Binder: 1923-1925 General Ads \#1

Slogan: Kodak as you Go

Subject(s): Woman and two men.

Text: All roads lead to pictures--the quiet lane just as surely as

the busy highway. With your Kodak tucked beside you, you have

only to pick and choose--and press the button.

Implied Audience: women / travelers

Product: autographic Kodak

Photo or Illustration Based: photo

Color or Black and White: bw
Description / Notes: Photo of a woman and two men sitting in a car, the woman is photographing boats in the harbor.

Binder: 1923-1925 General Ads \#1

Slogan: All Out-doors invites your Kodak

Subject(s): young man, woman fishing

Text: none

Implied Audience: outdoorsmen

Product: autographic Kodak

Photo or Illustration Based: photo

Color or Black and White: bw

Description / Notes: Photo of a couple at a waterfall, the man with fishing equipment, the woman with a Kodak.

Binder: 1923-1925 General Ads \#

Slogan: A Brownie Belongs

Subject(s): two boys

Text: It's fun sailing boats--it's more fun sailing boats and making pictures too. That's the beauty of a Brownie. On the diamond, at the lake, on the track--this simply-worked, Eastman-made camera adds to the sport at the time and gives you pictures that store up fun for the future. Whatever it is you like to do best, a Brownie belongs.

Implied Audience: parents/children

Product: Brownie

Photo or Illustration Based: photo

Color or Black and White: bw

Description / Notes: Photograph of two boys sailing toy boats.

One is photographing the other.

Binder: 1923-1925 General Ads \#1

Year: 1923

Slogan: Eastman Organic Chemicals Nitron

Subject(s): product - Chemicals

Product: Eastman Organic Chemicals

Color or Black and White:

Binder: 1923-1925 General Ads \#1

Year: 1923 
Slogan: Sulfosalicylic Acid A Reagent for Albumin

Subject(s): product - Chemicals

Product: Eastman Organic Chemicals

Color or Black and White:

Binder: 1923-1925 General Ads \#1

Year: 1923

Slogan: Eastman Organic Chemicals Thioacetic Acid

Subject(s): product - Chemicals

Product: Eastman Organic Chemicals

Color or Black and White:

Binder: 1923-1925 General Ads \#1

Year: Mar-23

Slogan: Eastman Organic Chemicals

Subject(s): product - Chemicals

Product: Eastman Organic Chemicals

Color or Black and White:

Binder: 1923-1925 General Ads \#1

Year: 1923

Slogan: For the Best Results-- Your Darkroom Should Have

Subject(s): product - medical

Product: Darkroom equipment

Description / Notes: Medical darkrooms.

Binder: 1923-1925 General Ads \#1

Year: 1923

Slogan: A Suggestion--

Subject(s): product - medical

Product: Dental X-Rays

Color or Black and White:

Binder: 1923-1925 General Ads \#1

Year: 1923

Slogan: Eastman Dupli-Tized X-Ray Films

Subject(s): product - medical

Product: Duplitized X-Ray Films

Color or Black and White:
Binder: 1923-1925 General Ads \#1

Year: Apr-23

Slogan: Dependability, Eastman Dupli-Tized X-Ray Films

Subject(s): product - medical

Product: Duplitized X-Ray Films

Color or Black and White:

Binder: 1923-1925 General Ads \#1

Year: May-23

Slogan: Clinical Photography - A necessity in modern medical practice.

Subject(s): product - medical

Product: Eastman Clinical Camera Outfit

Description / Notes: Price: $\$ 160.00$

Binder: 1923-1925 General Ads \#1

Year: 1923

Slogan: Eastman Universal Colorimeter

Subject(s): product - misc.

Product: Eastman Universal Colorimeter

Description / Notes: Caption reads: "Color measurement made

easy" and "Numerical specification of color made simple"

Binder: 1923-1925 General Ads \#1

Year: Apr-23

Slogan: Picture Stories

Subject(s): product - camera

Product: Kodak

Description / Notes: Autographic Kodaks \$6.50 and up

Binder: 1923-1925 General Ads \#1

Year: Jun-23

Slogan: Kodak

Subject(s): product - camera

Product: Kodak

Description / Notes: Autographic Kodaks \$6.50 and up

Binder: 1923-1925 General Ads \#1 
Year: Jul-23

Slogan: For The Fourth Load with Kodak Film and Shoot with a Kodak

Subject(s): product - camera

Product: Kodak

Description / Notes: Autographic Kodaks \$6.50 and up

Binder: 1923-1925 General Ads \#1

Year: Jul-23

Slogan: Every good time is a good time to Kodak

Subject(s): product - camera

Product: Kodak

Description / Notes: Autographic Kodaks \$6.50 and up

Binder: 1923-1925 General Ads \#1

Slogan: Take a Kodak with You

Subject(s): Boy

Text: You'll want pictures of the canoe trip, the hike, of all the fun that summer has in store and it's all easy with a Kodak or Brownie.

The rest of the gang will want to see the pictures you make and you'll be proud to show them. Remember the time that Bill found the hornets' nest and the hornets found bill? Next time have a

Kodak along and keep all the story--even to the date and title.

Implied Audience: parents/children

Product: Kodak / brownie

Photo or Illustration Based: photo

Color or Black and White: bw

Description / Notes: Photograph of a boy with a camera, sitting beside a canoe.

Binder: 1923-1925 General Ads \#1

Year: Mar-23

Slogan: No. 1 Pocket Kodak, Series II

Subject(s): product - camera

Product: No. 1 Pocket Kodak Series II

Color or Black and White:

Binder: 1923-1925 General Ads \#1

Year: May-23
Slogan: No. 1 Pocket Kodak, Series II Fixed focus model.

Subject(s): product - camera

Product: No. 1 Pocket Kodak Series II

Description / Notes: Price: 13.50

Binder: 1923-1925 General Ads \#1

Year: Apr-23

Slogan: Autographic Brownies

Subject(s): product - camera

Product: No. 2C Autographic Brownie.

Description / Notes: 2c Autographic Brownie price: \$15.00 Four sizes of Autographic Brownies priced from $\$ 9.00$ up.

Binder: 1923-1925 General Ads \#1

Year: Apr-23

Slogan: Announcing a new Kodak

Subject(s): product - camera

Product: No. 2C Autographic Kodak Special

Description / Notes: Price: $\$ 65.00$

Binder: 1923-1925 General Ads \#1

Year: Jul-23

Slogan: Two New Kodak Models

Subject(s): product - camera

Product: Nos. 1 and 1 A Pocket Kodaks Series II

Description / Notes: No. 1 Pocket Kodak Price: \$20.00 No.1A

Pocket Kodak Price: $\$ 22.00$

Binder: 1923-1925 General Ads \#

Slogan: There's a photographer in your town

Subject(s): none

Text: The bride or graduate will make an appointment with her photographer as a matter of course. Not that the giving of a portrait needs an occasion. At any time it's a gentle, graceful way of acknowledging friendships.

Product: photograph

Photo or Illustration Based: Text: Color or Black and White: bw

Description / Notes: No photo - text only. 
Binder: 1923-1925 General Ads \#1

Year: May-23

Slogan: The Premo Camera

Subject(s): product - camera

Product: Premo

Description / Notes: Sales

Binder: 1923-1925 General Ads \#1

Year: 1923

Slogan: Because They're Better

Subject(s): product - camera

Product: Special Kodaks

Description / Notes: Prices: $\$ 50.00$ and up at your dealers.

Binder: 1923-1925 General Ads \#1

Year: Jun-23

Slogan: Eastman Prepared X-Ray Developer and Fixing Powders

Subject(s): product - medical

Product: X-Ray developer and fix.

Color or Black and White:

Binder: 1923-1925 General Ads \#2

Slogan: Just what I wanted--A Kodak

Subject(s): Kodak Girl

Text: none

Implied Audience: women / Christmas

Product: autographic Kodak

Photo or Illustration Based: photo

Color or Black and White: bw

Description / Notes: Photo of a woman with a new camera--

Christmas presents on the table. (might be pregnant?)

Binder: 1923-1925 General Ads \#2

Slogan: Wear a vest pocket Kodak on your belt.

Subject(s): man in a canoe

Text: Vest pocket autographic Special, focusing model, is excellent

photographic equipment for any sportsman...

Implied Audience: outdoorsmen
Product: autographic Kodak

Photo or Illustration Based: photo

Color or Black and White: bw

Description / Notes: Photograph of a man in a canoe with his Kodak attached to his belt.

Binder: 1923-1925 General Ads \#2

Year: Oct-23

Slogan: Autographic Kodak

Subject(s): Man on a cow

Text: Good pictures, easily made, and complete even to date and

title written on the film at the time by means of the exclusively

Eastman autographic feature-- that's the Kodak way. You'll want

such pictures of live stock, crops, equipment, buildings, for

reference, and year-to-year comparison you'll point to them proudly in your Kodak album when they are made just for fun, like the illustration above.

Implied Audience: farmers

Product: autographic Kodak

Photo or Illustration Based: photo

Color or Black and White: bw

Publications: Farm

Description / Notes: Photograph of a man on a cow. Caption reads: "From a Kodak Negative Made on the Farm."

Binder: 1923-1925 General Ads \#2

Year: Dec-23

Slogan: A Kodak for Christmas

Subject(s): Mother, three children

Text: Hardly is it out of the package when it's out the door in happy hands, "clicking" the holiday story. Kodak is a gift that everyone wants.

Implied Audience: families / Christmas

Product: autographic Kodak

Photo or Illustration Based: photo

Color or Black and White: bw

Description / Notes: Photo of a mother photographing three children building a snowman. 
Binder: 1923-1925 General Ads \#2

Year: Nov-23

Slogan: Take a Kodak with You

Subject(s): Two men

Text: You'll find plenty of opportunity for a Kodak on your hunting

trip. And there are no game laws to stop the shot.

Implied Audience: hunters / outdoorsman

Product: autographic Kodak

Photo or Illustration Based: photo

Color or Black and White: bw

Description / Notes: Photo of two men, one handing his rifle to

the other, and pulling out his Kodak.

Binder: 1923-1925 General Ads \#2

Year: Oct-23

Slogan: Kodak on the Hunt

Subject(s): Woman and two men.

Text: There could be no more interesting trophies of the trip than clear, clean-cut pictures--the king a Kodak makes. Any Kodak is

compact to carry. Some are small enough to wear.

Implied Audience: outdoorsmen

Product: autographic Kodak

Photo or Illustration Based: photo

Color or Black and White: bw

Description / Notes: Photo of a woman photographing two men

with their hunting equipment, and dogs.

Binder: 1923-1925 General Ads \#2

Slogan: Take a Kodak with You

Subject(s): young man, woman

Text: And then as you press the button, the story that the happy

hours hold is yours for the years.

Implied Audience: travel

Product: autographic Kodak

Photo or Illustration Based: photo

Color or Black and White: bw

Description / Notes: Photograph of same woman in striped dress as above, but with a man with golf clubs. The woman is holding the camera.
Binder: 1923-1925 General Ads \#2

Year: Nov-23

Slogan: Bring Along A Brownie

Subject(s): father / son

Text: A day with Dad--there's a chance for pictures, and just the kind a Brownie likes to make. Incidentally, unless your dad knows about a Brownie he's going to be a little bit surprised when you show him the prints. "Why these are fine son," He says, and his face registers amazement. Well, why shouldn't they be fine? You and your Brownie made them.

Implied Audience: parents/children

Product: Brownie

Photo or Illustration Based: photo

Color or Black and White: bw

Description / Notes: Photo of a man in a canoe, posing as his son takes a photo.

Binder: 1923-1925 General Ads \#2

Year: Oct-23

Slogan: What you Want is a Brownie

Subject(s): three boys

Text: Sam makes the Jack-O'-lantern -- you make the picture.

Then in a day or so you both see the print. "Some photographer," Says Sam. And you are. A Brownie makes good picture-making easy.

Implied Audience: parents/children

Product: Brownie

Photo or Illustration Based: photo

Color or Black and White: bw

Description / Notes: Photo of a boy photographing two boys on a bench outside, one holding a pumpkin.

Binder: 1923-1925 General Ads \#2

Year: Sept-Oct 1923

Slogan: Bring Along A Brownie

Subject(s): three boys

Text: Ask your father how much he would give for pictures of the things he did when he was a boy--his pets, and sports and chums. 
Then tell him that the prices of Brownie cameras, made in Kodak shops by Kodak workmen, and easily worked, thoroughly capable picture-makers, begin at 2.00

Implied Audience: parents/children

Product: Brownie

Photo or Illustration Based: photo

Color or Black and White: bw

Description / Notes: Photo of a boy photographing two other

boys climbing a tree.

Binder: 1923-1925 General Ads \#2

Year: 1923

Slogan: Eastman Organic Chemicals - Xanthydrol

Subject(s): product - Chemicals

Product: Eastman Organic Chemicals

Color or Black and White: bw

Binder: 1923-1925 General Ads \#2

Year: 1923

Slogan: Eastman Organic Chemicals - Over 1600 Available.

Subject(s): product - Chemicals

Product: Eastman Organic Chemicals

Color or Black and White: bw

Binder: 1923-1925 General Ads \#2

Year: Aug-23

Slogan: Eastman Organic Chemicals Carbon Tetrachloride

Subject(s): product - Chemicals

Product: Eastman Organic Chemicals

Color or Black and White: bw

Binder: 1923-1925 General Ads \#2

Year: 1923

Slogan: Announcing a New Film--Eastman Dupli-Tized X-Ray Film

Super Speed

Subject(s): product - medical

Product: Duplitized X-Ray Films

Color or Black and White: bw
Binder: 1923-1925 General Ads \#2

Year: 1923

Slogan: "Radiography with Eastman Dental X-Ray Films"

Subject(s): product - medical

Product: Eastman Dental X-Ray Films

Color or Black and White: bw

Binder: 1923-1925 General Ads \#2

Year: Oct-23

Slogan: Three Things about Anastigmat Lenses

Subject(s): product - camera

Product: Kodak Anastigmat lens

Color or Black and White: bw

Binder: 1923-1925 General Ads \#2

Year: 1923

Slogan: Close-Ups with your Kodak

Subject(s): product - camera

Product: Kodak Portrait Attachment

Description / Notes: Price: \$1.25 and \$1.50

Binder: 1923-1925 General Ads \#2

Year: Jun-23

Slogan: No. 1 Autographic Kodak Special

Subject(s): product - camera

Product: No. 1 Autographic Kodak Special

Description / Notes: Price: $\$ 50.00$

Binder: 1923-1925 General Ads \#2

Year: 1923

Slogan: There are three New-Model Special Kodaks

Subject(s): product - camera

Product: No. 1 Autographic Kodak Special, No. 1A Autographic Kodak Special, No. 2C Autographic Kodak Special

Description / Notes: No. 1 Price: $\$ 50.00$ No. 1a Price: $\$ 60.00$ No. 2C Price: $\$ 65.00$

Binder: 1923-1925 General Ads \#2

Year: Sep-23 
Slogan: No. 1a Autographic Kodak Special

Subject(s): product - camera

Product: No. 1a Autographic Kodak Special

Description / Notes: Price: $\$ 60.00$

Binder: 1923-1925 General Ads \#2

Year: Sep-23

Slogan: No. 1a Autographic Kodak Special - A New Model in a

Popular Size

Subject(s): product - camera

Product: No. 1a Autographic Kodak Special

Color or Black and White:

Binder: 1923-1925 General Ads \#2

Year: Jul-23

Slogan: A new model--always in focus

Subject(s): product - camera

Product: No. 1a Pocket Kodak Series II

Description / Notes: Price $\$ 15.00$

Binder: 1923-1925 General Ads \#2

Year: 1923

Slogan: There's bound to be fun with a Brownie

Subject(s): product - camera

Product: No. 2C Autographic Brownie.

Description / Notes: Price: $\$ 15.00$ Four sizes of Autographic

Brownies: $\$ 9.00$ and up. Box type Brownies: $\$ 2.00$ and up.

Binder: 1923-1925 General Ads \#2

Year: Nov-23

Slogan: There's a photographer in your town

Subject(s): none

Text: Portraits for Christmas Your friends can buy anything you

can give them--except your photograph. Phone your photographer

now.

Implied Audience: Christmas

Product: photograph

Photo or Illustration Based: Text: Color or Black and White: bw
Description / Notes: No photo - text only.

Binder: 1923-1925 General Ads \#2

Year: 1923

Slogan: People Want the Premo

Subject(s): product - camera

Product: Premo

Description / Notes: Sales

Binder: 1923-1925 General Ads \#2

Year: 1923

Slogan: An Eastman-made Premium The Premo Camera

Subject(s): product - camera

Product: Premo

Description / Notes: Sales

Binder: 1923-1925 General Ads \#2

Year: Jun-23

Slogan: Offer the Premo

Subject(s): product - camera

Product: Premo

Color or Black and White: Publications: Novelty News

Description / Notes: Sales

Binder: 1923-1925 General Ads \#2

Year: Jul-23

Slogan: Premos for Premiums

Subject(s): product - camera

Product: Premo

Description / Notes: Sales

Binder: 1923-1925 General Ads \#2

Year: 1923

Slogan: For Better Prints for You

Subject(s): product - film \& paper

Product: Velox paper

Color or Black and White:

Binder: 1923-1925 General Ads \#3 
Year: Feb-24

Slogan: All Out-doors invites your Kodak

Subject(s): Kodak girls

Text: none

Implied Audience: women / travelers

Product: Autographic Kodak

Photo or Illustration Based: photo

Color or Black and White: bw

Description / Notes: Photo of two women, one in stripes, one in checks. One sitting on a fence, holding a camera, the other pointing at something.

Binder: 1923-1925 General Ads \#3

Year: Feb-24

Slogan: Kodak on the Farm

Subject(s): Young man, woman horse and carriage.

Text: Winter sets the stage for picture stories you will be glad to get with a Kodak. That's pleasure. But Kodak plays part in the business side of life on the farm, as well. There are pictures that sell cattle, horses, sheep, hogs; there are pictures that record crops, equipment, buildings--pictures worth the making, all of them. And the Kodak way is the easy way and one that's fun from the start.

Implied Audience: farmers

Product: autographic Kodak

Photo or Illustration Based: photo

Color or Black and White: bw

Description / Notes: Same photo as above, with horse and

sleigh, but with different text.

Binder: 1923-1925 General Ads \#3

Year: Feb-24

Slogan: Take a Kodak with You

Subject(s): Young man, woman horse and carriage.

Text: Whether city stroll of country hike, winter sets the stage for a picture worth the making. And the Kodak way is the easy way

and one that's fun from the start.

Implied Audience: travelers

Product: autographic Kodak
Photo or Illustration Based: photo

Color or Black and White: bw

Description / Notes: Photo of a couple, woman holding the

camera, photographing horse-drawn sled with logs on it.

Binder: 1923-1925 General Ads \#3

Year: Dec-23

Slogan: Childhood's kaleidoscope never waits and rarely repeats.

Have your Kodak ready!

Subject(s): Family

Text: none

Implied Audience: families

Product: Brownie

Photo or Illustration Based: photo

Color or Black and White: bw

Description / Notes: Photo of a father pulling two children on a

sled, while mother photographs.

Binder: 1923-1925 General Ads \#3

Year: Dec-23

Slogan: Just what you want--a Brownie

Subject(s): Father, two children

Text: And picture making is one sport that you and your dad can enjoy together.

Implied Audience: parents/children /Christmas

Product: Brownie

Photo or Illustration Based: photo

Color or Black and White: bw

Description / Notes: photo of father standing over a young boy holding a Brownie. Christmas tree, toys in the BG.

Binder: 1923-1925 General Ads \#3

Year: Feb-24

Slogan: Bring Along A Brownie

Subject(s): three boys

Text: It's fun to ski, and fun to hike, but it's more fun still with a

Brownie. The rest of the bunch can hardly wait to see the pictures you made. And they're so good that you, yourself, can hardly wait to show them. 
Implied Audience: parents/children

Product: Brownie

Photo or Illustration Based: photo

Color or Black and White: bw

Description / Notes: photo of three boys, one photographing the

other two making a fire, outside in Winter.

Binder: 1923-1925 General Ads \#3

Year: Dec-23

Slogan: Just what I wanted--a Brownie

Subject(s): two girls, parents in the bg

Text: Think of the fun you'll have with your own camera. Better

tell mother or tip off Dad that this easily-worked Eastman-made

camera is just exactly what you want.

Implied Audience: parents/children/Christmas

Product: Brownie cameras

Photo or Illustration Based: photo

Color or Black and White: bw

Description / Notes: Photo of two young girls, one holding

Brownie, Christmas tree, presents in the BG, as well as parents.

Binder: 1923-1925 General Ads \#3

Year: Oct-23

Slogan: Eastman Organic Chemicals

Subject(s): product - Chemicals

Product: Eastman Organic Chemicals

Description / Notes: Shows a chart of five years progress

Binder: 1923-1925 General Ads \#3

Year: Nov-23

Slogan: Eastman Organic Chemicals Quality Specifications

Subject(s): product - Chemicals

Product: Eastman Organic Chemicals

Color or Black and White:

Binder: 1923-1925 General Ads \#3

Year: Jan-24

Slogan: The Use of Eastman Organic Chemicals

Subject(s): product - Chemicals
Product: Eastman Organic Chemicals

Description / Notes: List No. 10

Binder: 1923-1925 General Ads \#3

Slogan: A Specific Test for Albumin

Subject(s): product - Chemicals

Product: Eastman Organic Chemicals

Color or Black and White

Binder: 1923-1925 General Ads \#3

Year: 1924

Slogan: Motion Pictures

Subject(s): product - camera

Product: Ciné-Kodak

Description / Notes: Caption: "You press the button" and

"Operating with Kodak Motor Drive"

Binder: 1923-1925 General Ads \#3

Year: Feb-24

Slogan: The Kodak Way

Subject(s): product - camera

Product: Ciné-Kodak

Description / Notes: Caption: "We Do the Rest" Price: \$335.00

Binder: 1923-1925 General Ads \#3

Year: Nov-23

Slogan: Darkroom Design

Subject(s): product - medical

Product: Darkroom plans

Description / Notes: "Complete darkroom plans, photographs, and equipment data will be sent free on request."

Binder: 1923-1925 General Ads \#3

Year: Aug-23

Slogan: Eastman Dupli-Tized Films Super Speed

Subject(s): product - medical

Product: Duplitized X-Ray Films

Color or Black and White: 
Binder: 1923-1925 General Ads \#3

Year: Jan-24

Slogan: Eastman Dupli-Tized Super Speed Films

Subject(s): product - medical

Product: Duplitized X-Ray Films

Color or Black and White:

Binder: 1923-1925 General Ads \#3

Year: 1924

Slogan: "Angulation" Becomes extremely easy with Eastman

Dental X-Ray Films

Subject(s): product - medical

Product: Eastman Dental X-Ray Films

Color or Black and White:

Binder: 1923-1925 General Ads \#3

Year: Oct-23

Slogan: A New Booklet, Radiography with Eastman Dental X-Ray Films

Subject(s): product - medical

Product: Eastman Dental X-Ray Films

Color or Black and White:

Binder: 1923-1925 General Ads \#3

Year: Nov-23

Slogan: Reliability, Eastman Dental X-Ray Films

Subject(s): product - medical

Product: Eastman Dental X-Ray Films

Color or Black and White:

Binder: 1923-1925 General Ads \#3

Year: Jan-24

Slogan: Detail is Assured With Eastman Dental X-Ray Films

Subject(s): product - medical

Product: Eastman Dental X-Ray Films

Color or Black and White:

Binder: 1923-1925 General Ads \#3

Year: Dec-23
Slogan: Eastman Universal Colorimeter

Subject(s): product - misc.

Product: Eastman Universal Colorimeter

Color or Black and White:

Binder: 1923-1925 General Ads \#3

Year: Feb-24

Slogan: Kodak in the Home

Subject(s): Mother father, child

Text: A Kodak record of the children catches them just as they are and keeps them just as they were.

Implied Audience: families

Product: Kodak

Photo or Illustration Based: photo

Color or Black and White: bw

Description / Notes: photo of father fixing a doll carriage while young girl watches, mother takes photos.

Binder: 1923-1925 General Ads \#3

Year: Jan-24

Slogan: A Kodak for the Farm

Subject(s): Young boy with skates

Text: In addition to the dollars-and-cents value that Kodak

pictures of cattle, horses, hogs, crops, equipment, buildings, have in the business of farming, Kodak plays part the year 'round in the farm's fun--as the picture above shows.

Implied Audience: farmers

Product: Kodak

Photo or Illustration Based: photo

Color or Black and White: bw

Description / Notes: Photo of a boy carrying skates, walking

through the snow. Caption reads: "When skating pond takes Tom from kindling pile, there's a chance for a story-telling picture. Such pictures are sure of a smile when you turn the page in your Kodak Album. Illustration from a Kodak Magazine"

Binder: 1923-1925 General Ads \#3

Year: Feb-24

Slogan: "Wait till I load my Brownie" 
Subject(s): two boys

Text: Of course he wants his Brownie along. He wished he had it with him the other day when Joe Herbert took up fancy skating one moment and sat down hard--the next. There's always more fun with a Brownie--and you'll get good pictures from the first.

Implied Audience: parents/children

Product: Kodak / brownie

Photo or Illustration Based: photo

Color or Black and White: bw

Description / Notes: Photo of a boy loading his Brownie, indoors, while a friend holding skates, in winter clothes waits.

Binder: 1923-1925 General Ads \#3

Year: Feb-24

Slogan: No. 1a Pocket Kodak Series II

Subject(s): product - camera

Product: No. 1a Pocket Kodak Series II

Description / Notes: Price: $\$ 11.00$

Binder: 1923-1925 General Ads \#3

Year: 1924

Slogan: Two Premiums in One

Subject(s): product - film \& paper

Product: No. 2 Film Pack Hawk-Eye, Kodakery

Description / Notes: Sales

Binder: 1923-1925 General Ads \#3

Year: Feb-24

Slogan: As a Premium--A Premo

Subject(s): product - camera

Product: Premo

Color or Black and White: Publications: Novelty News

Description / Notes: Sales

Binder: 1923-1925 General Ads \#3

Slogan: A Nationally Known Product for a Premium

Subject(s): product - camera

Product: Premo

Description / Notes: Sales
Binder: 1923-1925 General Ads \#3

Year: Nov-23

Slogan: A Premium with wide appeal

Subject(s): product - camera

Product: Premo Camera

Description / Notes: Sales

Binder: 1923-1925 General Ads \#3

Year: Jan-24

Slogan: If your photographic print was made on Velox the name is on the back.

Subject(s): product - film \& paper

Product: Velox paper

Color or Black and White:

Binder: 1923-1925 General Ads \#3

Year: Feb-24

Slogan: Look for "Velox" on the Back

Subject(s): product - film \& paper

Product: Velox paper

Color or Black and White:

Binder: 1923-1925 General Ads \#3

Year: Oct-23

Slogan: Eastman X-Ray Illuminator

Subject(s): product - medical

Product: X-Ray illuminator

Description / Notes: Price: $8 \times 10--\$ 6.00$ 14x17--\$16.50

Binder: 1923-1925 General Ads \#4

Year: May-24

Slogan: 1a Autographic Kodak Special Take this Kodak with you

Subject(s): product - camera

Product: 1a Autographic Kodak Special

Description / Notes: Price: $\$ 60.00$

Binder: 1923-1925 General Ads \#4

Year: Jun-24 
Slogan: The Speed You Need.

Subject(s): product - camera

Product: 1a Autographic Kodak Special

Color or Black and White:

Binder: 1923-1925 General Ads \#4

Year: May-24

Slogan: Keep a Kodak Story of the Children

Subject(s): Boy with chickens

Text: Then when years pass and Jimmie wears his hair short and his trousers long, you have him just as he was. It's all easy the

Kodak way--and fun from the first.

Implied Audience: families

Product: autographic Kodak

Photo or Illustration Based: photo

Color or Black and White: bw

Description / Notes: Photo of a boy looking in a chicken coop, with chicks. Caption reads "From a Kodak Negative"

Binder: 1923-1925 General Ads \#4

Year: Jun-24

Slogan: Take a Kodak with You

Subject(s): Family

Text: You'd like to remember a day like this and pictures won't let you forget. Any Kodak is convenient to carry, easy to work and fun

to use; you'll get good pictures from the first.

Implied Audience: families

Product: Autographic Kodak

Photo or Illustration Based: photo

Color or Black and White: bw

Description / Notes: Photograph of a man, woman and child

standing in a meadow, mother holding flowers, father

photographing child sitting in the field

Binder: 1923-1925 General Ads \#4

Year: April, 1924

Slogan: Kodak Picture-records

Subject(s): Farmer

Text: Kodak pictures play a definite part in your business. The illustration above, for example, plus the title and date you wrote on the film at the time by means of the autographic feature, is a complete picture-record. Each year you'll find it invaluable for reference. The autographic Kodak gives you just the pictures you want--gives them the easiest way and the cost is little enough.

Implied Audience: farmers

Product: Autographic Kodak

Photo or Illustration Based: photo

Color or Black and White: bw

Description / Notes: Photograph of a man on a horse-drawn farming machine. Caption reads: "'Harrowing Apr 7, '23' --picture and title from an autographic Kodak negative."

Binder: 1923-1925 General Ads \#4

Year: Apr-24

Slogan: Take a Kodak with You

Subject(s): four men

Text: Days like this, that you've looked forward to, offer pictures that you'll turn back to, again and again in your album. And any

Kodak is compact to carry, easy to work and fun to use.

Implied Audience: outdoorsmen

Product: Autographic Kodak

Photo or Illustration Based: photo

Color or Black and White: bw

Description / Notes: Photograph of four men with camping

equipment, canoe at the edge of a river.

Binder: 1923-1925 General Ads \#4

Slogan: Kodak on the Farm

Subject(s): Horses

Text: With spring plowing done and the team in pasture for a wellearned Sunday's rest, there's a chance for a Kodak picture. Storytelling pictures like this are plenty on the farm--you'll always be glad you have them. And for practical use in advertising and selling your cattle, horses, hogs, pictures are of obvious worth. It's all easy, and fun, the Kodak way.

Implied Audience: farmers

Product: autographic Kodak

Photo or Illustration Based: photo 


\section{Color or Black and White: bw}

Description / Notes: Photo of three horses.

Binder: 1923-1925 General Ads \#4

Year: May-24

Slogan: Keep a Kodak Story of the Children

Subject(s): mother / child

Text: none

Implied Audience: families

Product: Autographic Kodak

Photo or Illustration Based: photo

Color or Black and White: bw

Description / Notes: Photograph of a mother about to take a

photo of her baby in a carriage.

Binder: 1923-1925 General Ads \#4

Year: Apr-24

Slogan: Brownie cameras make good pictures

Subject(s): Two boys

Text: The rest of the bunch will want to see the pictures your

Brownie made--and you'll be proud to show them.

Implied Audience: parents/children

Product: autographic Kodak

Photo or Illustration Based: photo

Color or Black and White: bw

Description / Notes: Photo of two boys looking at photographs.

One has camera on his shoulder.

Binder: 1923-1925 General Ads \#4

Year: Jul-24

Slogan: Have your Kodak ready

Subject(s): Two men camping

Text: none

Implied Audience: outdoorsmen

Product: Autographic Kodak

Photo or Illustration Based: photo

Color or Black and White: bw

Description / Notes: Photo of two men on a rock, with camping equipment, one showing the other his camera. Caption reads: "See
Jim, how easy it is the Kodak way"

Binder: 1923-1925 General Ads \#4

Year: Mar-24

Slogan: Swing a Kodak from your shoulder

Subject(s): Two men fishing

Text: And then you take home the trip. It's all easy the Kodak way and you'll find it fun from the first.

Implied Audience: outdoorsmen

Product: Autographic Kodak

Photo or Illustration Based: photo

Color or Black and White: bw

Description / Notes: Photograph of two men standing at a river, one holding fish, and a pole, the other a Kodak.

Binder: 1923-1925 General Ads \#4

Year: Apr-24

Slogan: Kodak keeps the story

Subject(s): woman, captain of ship

Text: Just "click" the shutter of this easily-worked camera and to-

day's good times become to-morrow's good pictures.

Implied Audience: women / travelers

Product: Autographic Kodak

Photo or Illustration Based: photo

Color or Black and White: bw

Description / Notes: Photo of woman on a ship photographing

the captain at the wheel.

Binder: 1923-1925 General Ads \#4

Year: May-24

Slogan: Get the picture with a Brownie

Subject(s): two boys and a dog

Text: You think a lot of that dog of yours--and with Bud's

assistance he makes a fine subject for your camera. Pictures of

your friends, your sports, your trips--of whatever it is that you like to do best--are easy to get with a Brownie. Let them show you at the store.

Implied Audience: parents/children

Product: Brownie 
Photo or Illustration Based: photo

Color or Black and White: bw

Description / Notes: Photo of two boys, one holding a camera, the other posing the dog in a hat, on a box.

Binder: 1923-1925 General Ads \#4

Year: Feb-24

Slogan: Use Orcinol..., an Eastman Organic Chemical

Subject(s): product - Chemicals

Product: Eastman Organic Chemicals

Color or Black and White:

Binder: 1923-1925 General Ads \#4

Year: Mar-24

Slogan: Use Hydroxylamine Hydrochloride Eastman Standard

Subject(s): product - Chemicals

Product: Eastman Organic Chemicals

Color or Black and White:

Binder: 1923-1925 General Ads \#4

Year: Apr-24

Slogan: A new list of Eastman Organic Chemicals

Subject(s): product - Chemicals

Product: Eastman Organic Chemicals

Description / Notes: List No. 11

Binder: 1923-1925 General Ads \#4

Year: Apr-24

Slogan: Use Nitron, an Eastman Organic Chemical

Subject(s): product - Chemicals

Product: Eastman Organic Chemicals

Color or Black and White:

Binder: 1923-1925 General Ads \#4

Slogan: You press the button; we do the rest

Subject(s): Family, with maid.

Text: Cine-Kodak, the new Eastman invention, and its projector, the Kodascope, enable you to show in motion on your screen the sort of pictures you turn to first in your album. Train the camera, press the button and the result is a reel of personal motion pictures, obtained with characteristic Kodak ease, that for you and yours will be priceless. And the fascination of personal photoplays isn't all. Through Kodascope Libraries, Inc., professional releases may be rented for projection in your own home. Price of complete outfit, Cine-Kodak with either motor drive or tripod and crank Kodascope, Screen, etc., $\$ 335$. Cost of operating is less than $1 / 5$ of the operating expense of equipment using standard width film, and your finishing by Eastman experts in Eastman laboratories is paid for when you buy the film. You press the button; we do the rest.

\section{Implied Audience: families}

Product: Cine-Kodak

Photo or Illustration Based: photo

Color or Black and White: bw

Description / Notes: photograph of a man filming his children being served snacks outdoors by a maid, while the mother watches. Caption reads: "Making motion pictures with a CinéKodak (the motor cranks the camera)

Binder: 1923-1925 General Ads \#4

Year: May-22

Slogan: Motion pictures the Kodak Way

Subject(s): men in canoes

Text: Rushing water, swirl of spray, flashing paddles--press the button on your Ciné-Kodak and you're getting it all in motion. And then back from the trip, you have only to turn the switch on your Kodascope and once more the racing canoes swoop past you and the thrill of the moment is yours again. Nor are you limited to personal motion pictures of your favorite sports, your vacation trip, or the children. Professional releases--dramas, comedies, etc.-may be rented from Kodascope Libraries, Inc. and projected in your own home. ...

Implied Audience: outdoorsmen

Product: Cine-Kodak

Photo or Illustration Based: Photo

Color or Black and White: bw

Description / Notes: Photo of a man filming three canoes going down the river. Caption reads "The motor cranks the camera" 
Binder: 1923-1925 General Ads \#4

Year: Mar-24

Slogan: Ciné-Kodak for Motion Pictures

Subject(s): product - camera

Product: Ciné-Kodak

Description / Notes: Price: $\$ 335.00$ Caption reads: You press the button; we do the rest

Binder: 1923-1925 General Ads \#4

Year: Apr-24

Slogan: Speed in Exposure With--Eastman Dupli-Tized X-Ray

Films

Subject(s): product - medical

Product: Duplitized X-Ray Films

Color or Black and White:

Binder: 1923-1925 General Ads \#4

Year: Apr-24

Slogan: For Extra-Oral Radiographs Use-- Eastman Dupli-Tized XRay Films

Subject(s): product - medical

Product: Duplitized X-Ray Films

Color or Black and White:

Binder: 1923-1925 General Ads \#4

Year: Feb-24

Slogan: To Meet Every Need--Eastman Dental X-Ray Films

Subject(s): product - medical

Product: Eastman Dental X-Ray Films

Color or Black and White:

Binder: 1923-1925 General Ads \#4

Year: 1924

Slogan: Eastman Universal Colorimeter

Subject(s): product - Chemicals

Product: Eastman Universal Colorimeter

Description / Notes: An instrument to measure colors. Caption reads: Color measurement made easy, Numerical specification of color made simple

Binder: 1923-1925 General Ads \#4

Year: Mar-24

Slogan: Developer Economy with--Eastman X-Ray Developing

Powders

Subject(s): product - medical

Product: Eastman X-Ray Developing Powders

Color or Black and White:

Binder: 1923-1925 General Ads \#4

Year: Jun-24

Slogan: One Hundred Kodaks to One Hundred Scouts for One Hundred Pictures Big Kodak Competition Starts Now

Subject(s): product - camera

Product: No. 1a Pocket Kodak Series II

Description / Notes: Contest for Boy Scouts June 1, 1924-

October 1, 1924. Prizes for 100 best photographs.

Binder: 1923-1925 General Ads \#4

Year: Mar-24

Slogan: Demonstrated Pulling Power

Subject(s): product - camera

Product: No. 2 Film Pack Hawk-Eye

Color or Black and White:

Binder: 1923-1925 General Ads \#4

Slogan: Have your Kodak ready

Subject(s): Man, two women

Text: Picture-making is fun at the time and there's pleasure ahead--years of it--as you turn to the prints in your album.

Implied Audience: women / general

Product: photograph

Photo or Illustration Based: Text: Color or Black and White: bw

Description / Notes: photo of a man and woman having a picnic, a second woman photographs it.

Binder: 1923-1925 General Ads \#4 
Slogan: There's a photographer in your town

Subject(s): none

Text: The exchange of photographs at graduation time has rightly become a universal custom because it provides a graceful way of acknowledging friendships. In order that those who expect your

portrait may not be disappointed--Phone your photographer now.

Product: photograph

Photo or Illustration Based: photo

Color or Black and White: bw

Description / Notes: No photo - text only.

Binder: 1923-1925 General Ads \#4

Year: 1924

Slogan: Added Pulling Power

Subject(s): product - camera

Product: Premo

Description / Notes: Sales

Binder: 1923-1925 General Ads \#4

Year: Jun-24

Slogan: Velox

Subject(s): product - film \& paper

Product: Velox paper

Color or Black and White:

Binder: 1923-1925 General Ads \#5

Year: Aug-24

Slogan: Kodak at the Fair

Subject(s): Chicken, cow, and pig

Text: Fair time means vacation time to you and your family, and that means Kodak pictures as a matter of course. Everybody takes a Kodak on vacation. You'll make pictures of the picnic along the roadside, of prize stock at the fair grounds, of your own entries in county or state competitions. Then as the years go by, the prints in your Kodak album bring back the fun you had.

Implied Audience: farmers

Product: autographic Kodak

Photo or Illustration Based: photo

Color or Black and White: bw
Description / Notes: Photos of farm animals with caption: "pictures you'll prize"

Binder: 1923-1925 General Ads \#5

Year: Jul-24

Slogan: Take a Kodak with you

Subject(s): Kodak girls

Text: none

Implied Audience: women / travelers

Product: autographic Kodak

Photo or Illustration Based: photo

Color or Black and White: bw

Description / Notes: Photograph of two women sitting in a boat. Both have cameras, one is taking a picture.

Binder: 1923-1925 General Ads \#5

Year: Aug-24

Slogan: Take a Kodak with You

Subject(s): man and horse

Text: And then long after those happy vacation days you trip is

still before you--in your album. Any Kodak is compact to carry and easy to work. Stop in at your dealer's and see.

Implied Audience: outdoorsmen

Product: autographic Kodak

Photo or Illustration Based: photo

Color or Black and White: bw

Description / Notes: Photo of a man with a horse, standing beside a mountain, photographing.

Binder: 1923-1925 General Ads \#5

Year: Sep-24

Slogan: Take a Kodak with You

Subject(s): Man and woman

Text: none

Implied Audience: outdoors

Product: autographic Kodak

Photo or Illustration Based: photo

Color or Black and White: bw

Description / Notes: Photo of a man helping a woman with a 
Kodak up a steep trail.

Binder: 1923-1925 General Ads \#5

Year: Aug-24

Slogan: Let Kodak Keep the Story

Subject(s): Mother with four children

Text: What a chance for a picture! Yes, and your Kodak will make

the most of it. Vacation's fun is Kodak's opportunity.

Implied Audience: families / travel

Product: autographic Kodak

Photo or Illustration Based: photo

Color or Black and White: bw

Description / Notes: Photo of a mother photographing four

children playing in the sand at the waterside.

Binder: 1923-1925 General Ads \#5

Year: Oct-24

Slogan: Ready with your Kodak

Subject(s): two women and a man.

Text: And a split second is all you need for a picture you'll

treasure for years. That's picture making the Kodak way.

Implied Audience: outdoors

Product: autographic Kodak

Photo or Illustration Based: photo

Color or Black and White: bw

Description / Notes: Photo of two women posing with a stack of corn stalks in a field, while a man photographs them.

Binder: 1923-1925 General Ads \#5

Year: Oct-24

Slogan: Ready with your Kodak

Subject(s): Woman, two dogs

Text: Kennel and stable, house and grounds--all present endless

opportunity for a Kodak--your Kodak

Implied Audience: women

Product: autographic Kodak

Photo or Illustration Based: photo

Color or Black and White: bw

Description / Notes: Photo of a woman photographing two dogs on a bench in a garden.

Binder: 1923-1925 General Ads \#5

Year: Sep-25

Slogan: All Out-doors invites your Kodak

Subject(s): young man, woman

Text: none

Implied Audience: travel

Product: autographic Kodak

Photo or Illustration Based: photo

Color or Black and White: bw

Description / Notes: Photo of a man and woman standing on a rock at the waterside, the man is photographing.

Binder: 1923-1925 General Ads \#5

Year: Jun-24

Slogan: A Brownie for Vacation

Subject(s): boy and girl

Text: Vacation's almost here. Think of the fun you're going to

have--and the fun you're going to keep if you have a camera. Any Brownie is easy to use--whether it's box type or folding. Look one over at your Kodak dealer's and see for yourself. You'll get good pictures from the first.

Implied Audience: parents/children

Product: Brownie

Photo or Illustration Based: photo

Color or Black and White: bw

Description / Notes: Photo of a boy photographing a girl at the beach.

Binder: 1923-1925 General Ads \#5

Year: Jul-24

Slogan: Get your Brownie out

Subject(s): five boys

Text: Probably Fat Smith was concealing something up his sleeve beside muscle, but it was a great chance for your Brownie. That

picture will get a laugh every time you show it. It's all easy the Brownie way, and the finest sort of fun from the first. Let them show you at the store. Your dad would give a lot if he'd had a 
camera when he was your age. And Brownies cost little--in fact the prices start at $\$ 2$.

Implied Audience: parents/children

Product: Brownie

Photo or Illustration Based: photo

Color or Black and White: bw

Description / Notes: Photo of a boy photographing three boys gathered around another who is showing his muscle.

Binder: 1923-1925 General Ads \#5

Year: Aug-24

Slogan: Picture-making is fun from the first with a Brownie

Subject(s): Man, two boys.

Text: Uncle Frank was so interested in sailing Bob's boat that he never knew your Brownie made the picture till you showed him in the album. Then he was so tickled that he wanted an extra print for himself. Brownies are easy to work, cost little, and do a lot.

See for yourself at your Kodak dealer's.

Implied Audience: parents/children

Product: Brownie

Photo or Illustration Based: photo

Color or Black and White: bw

Description / Notes: Photo of a boy photographing another boy and his uncle sailing a toy boat.

Binder: 1923-1925 General Ads \#5

Year: 1924

Slogan: Widely Known Photographic Plants for Sale Under

Government Decree

Subject(s): product - misc.

Product: Camera factories in Rochester

Description / Notes: Ad for the sales of the Premo, and the

Folmer and Schwing Camera factories, Rochester, NY. August 15,

1924. Minimum prices: for Folmer \& Schwing--796,041,60. For

Premo--589,449,20

Binder: 1923-1925 General Ads \#5

Year: 1924

Slogan: Nearly 1800 Eastman Organic Chemicals
Subject(s): product - Chemicals

Product: Eastman Organic Chemicals

Color or Black and White:

Binder: 1923-1925 General Ads \#5

Year: 1924

Slogan: Quality specifications of Eastman Organic Chemicals

Subject(s): product - Chemicals

Product: Eastman Organic Chemicals

Color or Black and White:

Binder: 1923-1925 General Ads \#5

Year: May-24

Slogan: For Making--Standard Refractive Index Solutions

Subject(s): product - Chemicals

Product: Eastman Organic Chemicals

Color or Black and White:

Binder: 1923-1925 General Ads \#5

Year: Aug-25

Slogan: Your own movies

Subject(s): Man at horse-race

Text: The life and animation of the horse show crowds, the thrill of the jumps, the rhythmic swing of the winning tandem--press the button on your Ciné-Kodak and, with utmost ease, you're getting it all in motion ...

Implied Audience: outdoors

Product: Cine-Kodak

Photo or Illustration Based: photo

Color or Black and White: bw

Description / Notes: Photo of a man filming a horse race from

the stands. Caption reads: "You press the button; we do the rest."

Binder: 1923-1925 General Ads \#5

Year: Jul-24

Slogan: In Motion

Subject(s): Girl with two men on horse, many dogs

Text: The hunt is your hobby--the Ciné-Kodak keeps for you in

motion pictures all the story save the music of hounds and the 
tan-ta-ra--tan-ta-ra of the horn. Tennis and golf, bathing and yachting, fishing and hunting, horse show and race-meet--it's the action in each that gives the thrill. And the Ciné-Kodak tells and retells the story--in action. Just as it first made amateur photography popular by making it simple, Kodak now brings to you, in motion, personal pictures of the very things you care for most. And again it is done by the mere press of a button. You can now have personal movies with Kodak ease

Implied Audience: hunters / outdoorsman

Product: Ciné-Kodak

Photo or Illustration Based: photo

Color or Black and White: bw

Description / Notes: Photo of a woman filming two men on

horse, with more than ten dogs, leaving on a hunt. Caption reads:

"You press the button; we do the rest."

Binder: 1923-1925 General Ads \#5

Year: 1924

Slogan: Ciné-Kodak gets the picture in motion

Subject(s): product - camera

Product: Ciné-Kodak

Description / Notes: Price: $\$ 335.00$ Caption reads: You press

the button--the motor cranks the cmera-you're making motion

pictures--the easy Kodak way

Binder: 1923-1925 General Ads \#5

Year: 1924

Slogan: The Ciné-Kodak makes motion pictures

Subject(s): product - camera

Product: Ciné-Kodak

Description / Notes: Price: $\$ 335.00$ Caption reads: The Motor Cranks the Camera

Binder: 1923-1925 General Ads \#5

Year: Jul-24

Slogan: Get the scene in motion

Subject(s): Three boys fishing

Text: Life, action, the true spirit of the scene--Ciné-Kodak keeps it

all in motion pictures with typical Kodak ease. And afterwards, as you turn the switch on your Kodascope, you and your friends see the scene as you saw it first--in all it's vigourous action-- and the original thrill remains. ...

Implied Audience: parents/children

Product: Ciné-Kodak

Photo or Illustration Based: photo

Color or Black and White: bw

Description / Notes: Photo of three boys fishing, one netting a

fish, the other two filming and watching him.

Binder: 1923-1925 General Ads \#5

Year: Jul-24

Slogan: Action! And your Ciné-Kodak gets it al

Subject(s): two men on a dock

Text: The gentle whir of your Ciné-Kodak motor as it steadily

cranks the camera is lost in the roar of the onrushing hydroplane, but you're getting the scene in motion. And all you do is train the lens and press the button. That's motion pictures the Kodak way.

Implied Audience: outdoorsmen

Product: Ciné-Kodak

Photo or Illustration Based: photo

Color or Black and White: bw

Description / Notes: Photo of two men on a dock, watching a plane land in the water. Caption reads: "You press the button; we do the rest."

Binder: 1923-1925 General Ads \#5

Year: 1924

Slogan: Safety

Subject(s): product - medical

Product: Duplitized X-Ray Films

Color or Black and White:

Binder: 1923-1925 General Ads \#5

Year: May-24

Slogan: An announcement, To be available soon, Eastman Safety

Dupli-Tized X-Ray Films Super-Speed

Subject(s): product - medical 
Product: Duplitized X-Ray Films

Color or Black and White:

Binder: 1923-1925 General Ads \#5

Year: 1924

Slogan: Keep Your Radiographs in--Eastman Dental Film Mounts

Subject(s): product - medica

Product: Eastman Dental Film Mounts

Color or Black and White:

Binder: 1923-1925 General Ads \#5

Year: Jun-24

Slogan: Standard Technique in Dental Radiography

Subject(s): product - medica

Product: Eastman Dental X-Ray Films

Color or Black and White:

Binder: 1923-1925 General Ads \#5

Year: 1924

Slogan: Your Kodak in Europe

Subject(s): product - film \& paper

Product: film

Description / Notes: list of Kodak developers and printers in

Europe.

Binder: 1923-1925 General Ads \#5

Year: 1924

Slogan: Concessionaires! Salesboard Operators! Premium Users!

Use a Premo for Pulling Power

Subject(s): product - camera

Product: Hawk-eye and Premo

Description / Notes: Sales

Binder: 1923-1925 General Ads \#5

Year: 1924

Slogan: Concessionaires--Premium Users Sales board Operators

Subject(s): product - camera

Product: Hawk-eye and Premo

Description / Notes: sales
Binder: 1923-1925 General Ads \#5

Year: Sep-24

Slogan: This Boy Scout Wins a Kodak

Subject(s): product - camera

Product: No. 1a Pocket Kodak Series II

Description / Notes: Contest for Boy Scouts June 1, 1924-

October 1, 1924. Prizes for 100 best photographs.

Binder: 1923-1925 General Ads \#5

Year: 1924

Slogan: Demonstrated Pulling Power

Subject(s): product - camera

Product: No. 2 Film Pack Hawk-Eye

Description / Notes: Sales

Binder: 1923-1925 General Ads \#5

Year: 1924

Slogan: Concessionaires--Sales board Operators--Premium Users.

Use an Eastman Camera for Pulling Power

Subject(s): product - camera

Product: No. 2a Folding Cartridge Premo

Description / Notes: sales

Binder: 1923-1925 General Ads \#5

Year: Aug-24

Slogan: You too can win a $\$ 22$ Kodak

Subject(s): product - camera

Product: No.1a Pocket Kodak Series II

Description / Notes: Contest for Boy Scouts June 1, 1924-

October 1, 1924. Prizes for 100 best photographs.

Binder: 1923-1925 General Ads \#5

Year: Oct-24

Slogan: A new Kodak with a new shutter. Nos. 1a and 2c Kodaks Series III

Subject(s): product - camera

Product: Nos. $1 \mathrm{~A}$ and 2C Kodaks Series III

Description / Notes: Prices: 1A \$30.00 2C \$33.00 
Binder: 1923-1925 General Ads \#5

Year: 1924

Slogan: Everybody likes to take pictures

Subject(s): product - camera

Product: Premo

Description / Notes: Sales

Binder: 1923-1925 General Ads \#5

Year: Aug-24

Slogan: An Established Reputation for your Premium

Subject(s): product - camera

Product: Premo

Description / Notes: Sales

Binder: 1923-1925 General Ads \#6

Year: Nov-24

Slogan: Get your Kodak Out

Subject(s): Man, woman, three dogs

Text: An ideal time for picture making is autumn. Whether a hunt

or a hike, have your Kodak ready for the pictures you're sure to

want.

Implied Audience: hunters / outdoorsman

Product: autographic Kodak

Photo or Illustration Based: photo

Color or Black and White: bw

Description / Notes: Photo of a hunter posing with his three

dogs while a woman photographs them.

Binder: 1923-1925 General Ads \#6

Year: Oct-24

Slogan: You'll be glad you had your Kodak

Subject(s): Two men, three dogs

Text: No trouble to carry it--just put it in your pocket or swing it

from you shoulder; and think how you'll prize the pictures you

make. It's all easy the Kodak way, as your Kodak dealer will show

you.

Implied Audience: hunters / outdoorsman

Product: autographic Kodak
Photo or Illustration Based: photo

Color or Black and White: bw

Description / Notes: photograph of two hunters, one posing with three dogs while the other takes a photograph.

Binder: 1923-1925 General Ads \#6

Year: Dec-24

Slogan: A Brownie Gift Box

Subject(s): Boy

Text: You're ready for pictures right off the reel. Outfit includes a No. 2 Brownie Camera, two rolls of Kodak film, Eastman album,

Kodak portrait attachment, and blank for free year's subscription to the photographic magazine Kodakery

Implied Audience: parents/children

Product: Brownie

Photo or Illustration Based: photo-illustration

Color or Black and White: bw

Description / Notes: Photo of a boy with a new Brownie, box on the table. There is a drawing of a boy photographing his dog inset into the photo like a thought bubble.

Binder: 1923-1925 General Ads \#6

Year: Nov-24

Slogan: Brownie Gift Box

Subject(s): boy and girl

Text: A complete picture-taking, picture-keeping outfit--just what you'd like for Christmas. A barrel of photographic fun packed in a holiday box and you're ready to start enjoying it as soon as you lift the lid. Inside is everything the Junior photographer needs for pictures of his play and playmates--including equipment for "closeup" portraits at arm's distance--and an album for keeping the prints in tip-top shape. ...

Implied Audience: parents/children/Christmas

Product: Brownie

Photo or Illustration Based: photo

Color or Black and White: bw

Description / Notes: Photo of a boy with a Brownie in his hands, sister watching. Box sits on the desk beside him. 
Binder: 1923-1925 General Ads \#6

Year: Oct-24

Slogan: Every Boy wants a Brownie

Subject(s): four boys

Text: Chances are that your dad passes a Kodak dealer's store on his way from the office Chances are, too, that he would be glad to have you make pictures the easy Brownie way because he can enjoy them with you. Price, $\$ 2$ up, and good sport and good pictures from the start.

Implied Audience: parents/children

Product: Brownie

Photo or Illustration Based: photo

Color or Black and White: bw

Description / Notes: Photo of a three boys, one with a camera watching another boy do a handstand.

Binder: 1923-1925 General Ads \#6 Year: 1924

Slogan: Eastman Dintrosalicyic Acid

Subject(s): product - Chemicals

Product: Eastman Organic Chemicals

Color or Black and White:

Binder: 1923-1925 General Ads \#6

Year: Jul-24

Slogan: Announcing Eastman Low Viscosity Cotton

Subject(s): product - Chemicals

Product: Eastman Organic Chemicals

Color or Black and White:

Binder: 1923-1925 General Ads \#6

Year: Jul-24

Slogan: Low Viscosity Cotton for Automobile Lacquers

Subject(s): product - Chemicals

Product: Eastman Organic Chemicals

Color or Black and White:

Binder: 1923-1925 General Ads \#6

Year: Aug-24
Slogan: Use Eastman Pyrogallol for Gas Analysis

Subject(s): product - Chemicals

Product: Eastman Organic Chemicals

Color or Black and White:

Binder: 1923-1925 General Ads \#6

Year: Aug-24

Slogan: Eastman Cotton Forms a Tough Skin

Subject(s): product - Chemicals

Product: Eastman Organic Chemicals

Color or Black and White:

Binder: 1923-1925 General Ads \#6

Year: Aug-24

Slogan: For Medicinal use Eastman Carbon Tetrachloride

Subject(s): product - Chemicals

Product: Eastman Organic Chemicals

Color or Black and White:

Binder: 1923-1925 General Ads \#6

Year: Sep-24

Slogan: Eastman Organic Chemicals

Subject(s): product - Chemicals

Product: Eastman Organic Chemicals

Description / Notes: With graph charting growth over six years

Binder: 1923-1925 General Ads \#6

Year: Oct-24

Slogan: Kodalak Metal Lacquers

Subject(s): product - Chemicals

Product: Eastman Organic Chemicals

Color or Black and White:

Binder: 1923-1925 General Ads \#6

Year: Oct-24

Slogan: Kodalak Wood Lacquers

Subject(s): product - Chemicals

Product: Eastman Organic Chemicals

Color or Black and White: 
Binder: 1923-1925 General Ads \#6

Year: Dec-24

Slogan: Acetic Anhydride, 99-100\%, an Eastman Organic Chemical

Subject(s): product - Chemicals

Product: Eastman Organic Chemicals

Color or Black and White:

Binder: 1923-1925 General Ads \#6

Year: Nov-24

Slogan: And you made the movie yourself.

Subject(s): four men, one woman

Text: In your own home, on your own screen, you can show

movies that you made yourself with typical Kodak ease. A reel or two of your favorite sport, little dramas in which you and your friends star, movie chronicles of the children...

Implied Audience: families

Product: Cine-Kodak

Photo or Illustration Based: photo

Color or Black and White: bw

Description / Notes: Photo of a group of adults around a screen,

with a man running the projector, showing films.

Binder: 1923-1925 General Ads \#6

Year: Dec-24

Slogan: Movies of your Merry Christmas

Subject(s): five men skiing

Text: It's all easy with a Ciné-Kodak, and there's an unusual chance for movies now. Coasting, skiing, skating--the tang of winter speeds up the sports of winter. There's action galore at holiday time, and that's what you want for the screen. From the "spill" on the toboggan slide to the fancy skaters at the rink, press the button and you're making a movie of it; turn the switch on your Kodascope and you're showing it home on the screen. Movies you make yourself aren't all the story either. Through Kodascope Libraries, Inc., professional releases with such stars as Douglas Fairbanks, Charlie Chaplin, and Norma Talmadge may be secured at a modest rental for projection on your own screen.
Implied Audience: outdoors

\section{Product: Ciné-Kodak}

Photo or Illustration Based: photo

Color or Black and White: bw

Description / Notes: Photo of a man with Ciné-Kodak filming

four men in skis. Caption reads: "With the Ciné-Kodak you press the button; the motor cranks the camera

Binder: 1923-1925 General Ads \#6

Year: Sep-24

Slogan: Movies you make yourself

Subject(s): Woman, man with horse

Text: There's something new in photography--a new pleasure and a fascinating one--motion pictures the Kodak way. With the same ease that you make ordinary snapshots you can now film "movies" of your family, your friends, your sports and pleasures, and show them on screen in your own home. The camera is the Ciné-Kodak, and to operate it all you do is train the lens and press the button. A motor cranks the camera and gets the scene in action. The projector is the Kodascope--also Eastman made--and it not only allows you to show your own movies but professional releases as well...

Implied Audience: travel

Product: Ciné-Kodak

Photo or Illustration Based: photo

Color or Black and White: bw

Description / Notes: Photo of a woman with a movie camera, filming a man on a horse jumping over a fence.

Binder: 1923-1925 General Ads \#6

Year: 1924

Slogan: Quality and Safety are combined in the new Eastman

Safety Dupli-tized X-Ray Films

Subject(s): product - medical

Product: Duplitized X-Ray Films

Color or Black and White:

Binder: 1923-1925 General Ads \#6

Year: 1924 
Slogan: Detail in Radiographs

Subject(s): product - medical

Product: Duplitized X-Ray Films

Color or Black and White:

Binder: 1923-1925 General Ads \#6

Year: Nov-24

Slogan: For Every Technique, Use Eastman Dupli-Tized X-Ray

Films

Subject(s): product - medical

Product: Duplitized X-Ray Films

Color or Black and White:

Binder: 1923-1925 General Ads \#6

Year: 1924

Slogan: In the Practice of Orthodontia

Subject(s): product - medica

Product: Eastman Dental X-Ray Films

Color or Black and White:

Binder: 1923-1925 General Ads \#6

Year: Sep-24

Slogan: Radiographs show Impacted Molars

Subject(s): product - medical

Product: Eastman Dental X-Ray Films

Color or Black and White:

Binder: 1923-1925 General Ads \#6

Year: Nov-24

Slogan: Eastman Dental X-Ray Films Packet "C"

Subject(s): product - medical

Product: Eastman Dental X-Ray Films

Color or Black and White:

Binder: 1923-1925 General Ads \#6

Year: Sep-24

Slogan: An Eastman-made Roll-film Camera for Premium Users

Subject(s): product - camera

Product: Hawk-eye

\section{Description / Notes: Sales}

Binder: 1923-1925 General Ads \#6

Year: Aug-24

Slogan: Concessionaires--Premium Users Sales board Operators

Subject(s): product - camera

Product: Hawk-eye and Premo

Description / Notes: Sales

Binder: 1923-1925 General Ads \#6

Year: Oct-24

Slogan: Suit Yourself

Subject(s): product - camera

Product: Hawk-eye and Premo

Description / Notes: Sales

Binder: 1923-1925 General Ads \#6

Year: Nov-24

Slogan: An Eastman Camera is a Year-'round Premium

Subject(s): product - camera

Product: Hawk-eye and Premo

Description / Notes: Sales

Binder: 1923-1925 General Ads \#6

Year: Dec-24

Slogan: "From Dot and Daddy"--A Kodak

Subject(s): Mother, father, daughter

Text: It has been hard for Dot to keep the big secret but she

managed somehow, and mother is the most surprised person in the world. And pleased, too. A Kodak is just what she wanted.

Implied Audience: families / Christmas

Product: Kodak

Photo or Illustration Based: photo

Color or Black and White: bw

Description / Notes: Photo of a father taking a camera off of the tree, while young daughter covers mothers eyes, sitting in a chair.

Binder: 1923-1925 General Ads \#6

Year: Nov-24 
Slogan: Keep a Kodak Story of the Children

Subject(s): Mother, two young children.

Text: With children in your home, there certainly should be Kodak pictures in your album. And the time will come when you'll never forgive yourself if the chance slips by. Get a Kodak to-day. You'll

be making good pictures with it to-morrow.

Implied Audience: families

Product: Kodak

Photo or Illustration Based: photo

Color or Black and White: bw

Description / Notes: Photo of a mother taking a photograph of

two small children, one in a crib/playpen.

Binder: 1923-1925 General Ads \#6

Year: Dec-24

Slogan: All Outdoors invites your Christmas Kodak

Subject(s): Woman

Text: none

Implied Audience: women / travelers

Product: Kodak

Photo or Illustration Based: photo

Color or Black and White: bw

Description / Notes: Photo of a woman on the top of a hill,

wearing snowshoes, waving at the camera. Snowshoe tracks in the foreground.

Binder: 1923-1925 General Ads \#6

Year: Jan-25

Slogan: An Added Attraction

Subject(s): product - camera

Product: Kodakery

Description / Notes: Sales

Binder: 1923-1925 General Ads \#6

Year: 1924

Slogan: A Sensible Headliner for any sales scheme

Subject(s): product - camera

Product: No. 2 Cartridge Hawk-Eye.

Description / Notes: Sales
Binder: 1923-1925 General Ads \#6

Year: Nov-24

Slogan: There's a photographer in your town

Subject(s): None

Text: A gift inspired by sentiment and thus expressive of the true spirit of Christmas--your photograph.

Implied Audience: Christmas

Product: photograph

Photo or Illustration Based: Text: Color or Black and White: bw

Description / Notes: No photo - text only.

Binder: 1923-1925 General Ads \#6

Year: Aug-24

Slogan: Modern Radiography Demands Detail and Safety

Subject(s): product - medical

Product: X-Ray intensifying screens

Color or Black and White:

Binder: 1923-1925 General Ads \#6

Year: Sep-24

Slogan: Eastman X-Ray Intensifying Screens

Subject(s): product - medical

Product: X-Ray intensifying screens

Color or Black and White:

Binder: 1923-1925 General Ads \#7

Year: Feb-25

Slogan: Your Brownie gets the picture

Subject(s): Five boys

Text: All set for the bump, and in a second more, all upset. You and your Brownie get two pictures that hand you a laugh every time you look at them. Picture-making is lots of fun and easy from the start with a Brownie. Prices start in low-- $\$ 2$.

Implied Audience: parents/children

Product: Autographic Kodak

Photo or Illustration Based: photo

Color or Black and White: bw 
Description / Notes: photograph of a boy with a camera, photographing four boys on a sled, about to go down a hill.

Binder: 1923-1925 General Ads \#7

Year: May-25

Slogan: Take a Kodak with You

Subject(s): Man

Text: none

Implied Audience: travelers

Product: autographic Kodak

Photo or Illustration Based: photo

Color or Black and White: bw

Description / Notes: Photo of a man facing away from the camera, with Kodak on his shoulder, at a train(?) station.

Binder: 1923-1925 General Ads \#7

Year: Mar-25

Slogan: All Outdoors invites your Kodak

Subject(s): Man with a gun

Text: none

Implied Audience: hunters / outdoorsman

Product: autographic Kodak

Photo or Illustration Based: photo

Color or Black and White: bw

Description / Notes: Photo of a man from behind, with a gun

and a Kodak on his shoulder, in the snow.

Binder: 1923-1925 General Ads \#7

Year: Apr-25

Slogan: The Autographic Kodak Way

Subject(s): Man with horses

Text: Whether you're disliking the south lot, taking a Sunday trip, or stealing time to fish, today's Kodak snapshot is tomorrow's picture record. For in addition to the print that keeps the story there's the date and title you wrote on the film at the time-information you want for reference. All the Kodaks have this exclusively Eastman, autographic feature.

Implied Audience: farmers

Product: autographic Kodak
Photo or Illustration Based: photo

Color or Black and White: bw

Description / Notes: Photo of a man photographing two horses pulling a plow, with another man on it.

Binder: 1923-1925 General Ads \#7

Year: May-25

Slogan: Take a Kodak with You

Subject(s): Two men camping

Text: Every incident along the trail suggests a Kodak picture. It's sport at the moment--and sport again whenever you open the

album.

Implied Audience: outdoorsmen

Product: autographic Kodak

Photo or Illustration Based: photo

Color or Black and White: bw

Description / Notes: Photo of two men at a campsite, one photographing the other cooking dinner over a fire.

Binder: 1923-1925 General Ads \#7

Year: Feb-25

Slogan: Kodak Does Double Duty

Subject(s): two photos of a child

Text: Once the hilarious tot, now the serious six-year-old; here's a page for your Kodak album that will always be a genuine joy. From sun-up till evening chores are done you'll find chances a-plenty for story-telling pictures like these. And chances, as well, for picturerecords of cotton field and fruit orchard, of drove, herd and flock-pictures you'll value later for reference.

Implied Audience: families

Product: autographic Kodak

Photo or Illustration Based: photo

Color or Black and White: bw

Description / Notes: Two photos, of a baby and a young girl-the text implies that the images are of the same girl. Caption reads: "From a Kodak Negative"

Binder: 1923-1925 General Ads \#7

Year: Jan-25 
Slogan: Take a Kodak with You

Subject(s): Two women and a man

Text: When nature beckons, Kodak calls and you put the scene in a picture. It's all easy the Kodak way and pleasure a-plenty as well.

Implied Audience: outdoors / women

Product: autographic Kodak

Photo or Illustration Based: photo

Color or Black and White: bw

Description / Notes: Photo of a woman on skis photographing a woman and man, also on skis.

Binder: 1923-1925 General Ads \#7

Year: Jun-25

Slogan: All Outdoors invites your Kodak

Subject(s): Woman with umbrella

Text: none

Implied Audience: women / travelers

Product: autographic Kodak

Photo or Illustration Based: photo

Color or Black and White: bw

Description / Notes: Photo of a woman on the beach with

Kodak, walking while holding an umbrella / parasol.

Binder: 1923-1925 General Ads \#7

Year: Apr-25

Slogan: Picture Ahead, Kodak as you go.

Subject(s): Woman's arms

Text: None

Implied Audience: travelers

Product: autographic Kodak

Photo or Illustration Based: photo

Color or Black and White: bw

Description / Notes: Photo of a Kodak in the map pocket of a car, the door is open, and a woman with gloves is reaching in to get the camera out.

Binder: 1923-1925 General Ads \#7

Year: Jan-25
Slogan: Kodak on the Farm

Subject(s): Young girl

Text: Snapshots of the children that you make to-day you'll

treasure to-morrow. And pictures of stock and crops that you want now for advertising and selling, you'll find valuable later for reference.

Implied Audience: families / farmers

Product: autographic Kodak

Photo or Illustration Based: photo

Color or Black and White: bw

Description / Notes: Photo of a young girl working at a desk.

Caption reads: "Illustration from a negative made with an

Autographic Kodak"

Binder: 1923-1925 General Ads \#7

Year: Feb and March, 1925

Slogan: Ready with your Kodak

Subject(s): young man, woman

Text: There's a real thrill to the picture that breaks on the scene

without warning. Out comes your Kodak; quickly and easily you

bring it into play and -- the picture that found you prepared

Implied Audience: outdoors / women

Product: Autographic Kodak

Photo or Illustration Based: photo

Color or Black and White: bw

Description / Notes: Photo of a young couple in the snow, by a lake. The man is pulling his Kodak out of it's case on his shoulder

Binder: 1923-1925 General Ads \#7

Year: Apr-25

Slogan: "I'm bringing my Brownie"

Subject(s): Boy on a bike

Text: A Brownie doubles the day's fun; and once you have the

story in pictures, it's yours for keeps. That's what you'll get out of a Brownie. Here's that we put in, three big features. Folding advantage: you can always take along a Brownie, for it's never in the way. Autographic attachment: you can write the date and title on the film the minute you click the shutter. Eastman dependability: Brownies are made to stand up. 
Implied Audience: parents/children Product: Brownie

Photo or Illustration Based: photo

Color or Black and White: bw

Description / Notes: Photo of a boy with a Brownie on his

shoulder, on a bike riding away from the camera, his arm up waving.

Binder: 1923-1925 General Ads \#7

Year: May-25

Slogan: Keep your fun with a Brownie

Subject(s): boy with dog

Text: Now that Tige has learned the trick, it's no trick at all to make his picture. Every day, whatever you're doing, you'll find chances a-plenty for just such snapshots. It's like rolling off a log to "shoot" pictures with a Brownie. Open the camera, "click" the shutter, and you have your fun on the film.

Implied Audience: parents/children

Product: Brownie

Photo or Illustration Based: photo

Color or Black and White: bw

Description / Notes: Photograph of a boy with a camera,

photographing his dog.

Binder: 1923-1925 General Ads \#7

Year: Jun-25

Slogan: It's more fun with a Brownie

Subject(s): three boys

Text: Making the paddle wheel was fun, and so was making the picture. That's the Brownie's job--to get good pictures of your good times. Just open the camera, "click" the shutter and it's all over but the thrill you'll have when you see the print.

Implied Audience: parents/children

Product: Brownie

Photo or Illustration Based: photo

Color or Black and White: bw

Description / Notes: Photo of three boys wading in a river, with small waterfall, one photographing the other two with a paddle wheel.
Binder: 1923-1925 General Ads \#7

Year: Apr-25

Slogan: Brownie pictures "go big"

Subject(s): three boys and a horse

Text: Ed's pony-cart snapshot tickled the boys and, of course that tickled Ed. You'll get the same thrill when you show the fellows your Brownie pictures. And Brownies reduce photography to its simplest terms. Let the nearest Kodak dealer prove it to you.

Implied Audience: parents/children

Product: Brownie

Photo or Illustration Based: photo

Color or Black and White: bw

Description / Notes: Photo of a boy with a camera,

photographing two boys in a cart being pulled by a horse.

Binder: 1923-1925 General Ads \#7

Year: 1924

Slogan: Eastman Cotton for Better Finishes

Subject(s): product - Chemicals

Product: Eastman Organic Chemicals

Color or Black and White:

Binder: 1923-1925 General Ads \#7

Year: 1925

Slogan: Announcing Kodalak Furniture Finishes

Subject(s): product - Chemicals

Product: Eastman Organic Chemicals

Color or Black and White:

Binder: 1923-1925 General Ads \#7

Year: 1925

Slogan: Eastman Organic Chemicals

Subject(s): product - Chemicals

Product: Eastman Organic Chemicals

Color or Black and White:

Binder: 1923-1925 General Ads \#7

Year: 1925 
Slogan: Eastman Cotton

Subject(s): product - Chemicals

Product: Eastman Organic Chemicals

Color or Black and White:

Binder: 1923-1925 General Ads \#7

Year: Jan-25

Slogan: Use Eastman Organic Chemicals

Subject(s): product - Chemicals

Product: Eastman Organic Chemicals

Color or Black and White:

Binder: 1923-1925 General Ads \#7

Year: Jan-25

Slogan: There are 46 Indicators listed as Eastman Organic Chemicals

Subject(s): product - Chemicals

Product: Eastman Organic Chemicals

Color or Black and White:

Binder: 1923-1925 General Ads \#7

Year: Feb-25

Slogan: Kodalak Metal Finishes

Subject(s): product - Chemicals

Product: Eastman Organic Chemicals

Color or Black and White:

Binder: 1923-1925 General Ads \#7

Year: Mar-25

Slogan: There's a Kodalak for Every Purpose

Subject(s): product - Chemicals

Product: Eastman Organic Chemicals

Color or Black and White:

Binder: 1923-1925 General Ads \#7

Year: Mar-25

Slogan: Kodalak Furniture Finishes Rich and Durable

Subject(s): product - Chemicals

Product: Eastman Organic Chemicals

\section{Color or Black and White}

Binder: 1923-1925 General Ads \#7

Year: Mar-25

Slogan: Kodalak Black Lacquers

Subject(s): product - Chemicals

Product: Eastman Organic Chemicals

Color or Black and White:

Binder: 1923-1925 General Ads \#7

Year: 1924

Slogan: High Speed Radiography Reduces Movement

Subject(s): product - medical

Product: Duplitized X-Ray Films

Color or Black and White:

Binder: 1923-1925 General Ads \#7

Year: 1925

Slogan: Good X-Ray Films

Subject(s): product - medical

Product: Duplitized X-Ray Films

Color or Black and White:

Binder: 1923-1925 General Ads \#7

Year: 1925

Slogan: Shadow Detail

Subject(s): product - medical

Product: Duplitized X-Ray Films

Color or Black and White:

Binder: 1923-1925 General Ads \#7

Year: Dec-24

Slogan: For Screen Efficiency Eastman Dupli-Tized X-Ray Films Super-Speed

Subject(s): product - medical

Product: Duplitized X-Ray Films

Color or Black and White:

Binder: 1923-1925 General Ads \#7 
Year: 1925

Slogan: The Latest Eastman Aero Camera

Subject(s): product - camera

Product: Eastman Aero Camera

Color or Black and White:

Binder: 1923-1925 General Ads \#7

Year: 1925

Slogan: K-5 The Latest Eastman Aero Camera

Subject(s): product - camera

Product: Eastman Aero Camera, K-5

Color or Black and White:

Binder: 1923-1925 General Ads \#7

Year: 1925

Slogan: Eastman Aero Camera

Subject(s): product - camera

Product: Eastman Aero Camera, K-5

Color or Black and White:

Binder: 1923-1925 General Ads \#7

Year: 1924

Slogan: From every point of view--Eastman Dental X-Ray Films

Subject(s): product - medical

Product: Eastman Dental X-Ray Films

Color or Black and White:

Binder: 1923-1925 General Ads \#7

Year: 1925

Slogan: Eastman Dental X-Ray Films The World's Standard

Subject(s): product - medical

Product: Eastman Dental X-Ray Films

Color or Black and White:

Binder: 1923-1925 General Ads \#7

Year: 1925

Slogan: When you use Eastman Dental X-Ray Films

Subject(s): product - medical

Product: Eastman Dental X-Ray Films

\section{Color or Black and White:}

Binder: 1923-1925 General Ads \#7

Year: Mar-25

Slogan: It's fun with a Brownie

Subject(s): Boy

Text: Ted couldn't wait for the paint to dry; the moment he put

his brush down he got his Brownie out. And now he'll hardly sleep

till he shows the bunch the print. Brownies are cameras of the

easy-to-work, handy-to-carry, sure-fire kind. You start right in to

get good pictures; it's nothing but fun from the first.

Implied Audience: parents/children

Product: Folding Brownie.

Photo or Illustration Based: photo

Color or Black and White: bw

Description / Notes: photo of a boy photographing a toy boat

indoors on a workbench.

Binder: 1923-1925 General Ads \#7

Year: Jun-25

Slogan: No. 1a Pocket Kodak Series II - For the kind of pictures you like

Subject(s): product - camera

Product: No. 1a Pocket Kodak Series II

Description / Notes: Price: $\$ 26.00$

Binder: 1923-1925 General Ads \#7

Year: 1925

Slogan: To Promote Sales Use an Eastman Camera

Subject(s): product - camera

Product: No. 2 Cartridge Hawk-Eye.

Color or Black and White: Publications:

Description / Notes: Sales

Binder: 1923-1925 General Ads \#7

Year: 1925

Slogan: The Public Knows

Subject(s): product - camera

Product: No. 2 Cartridge Hawk-Eye. 


\section{Description / Notes: Sales}

Binder: 1923-1925 General Ads \#7

Year: Mar-25

Slogan: Boost Business with this camera

Subject(s): product - camera

Product: No. 2 Cartridge Hawk-Eye.

Description / Notes: Sales

Binder: 1923-1925 General Ads \#7

Year: Mar-25

Slogan: Center your sales scheme on an Eastman camera

Subject(s): product - camera

Product: No. 2 Cartridge Hawk-Eye.

Description / Notes: Sales

Binder: 1923-1925 General Ads \#7

Year: Apr-25

Slogan: Premos--Will please your customers

Subject(s): product - camera

Product: Premo

Description / Notes: Sales

Binder: 1923-1925 General Ads \#7

Year: 1925

Slogan: Look for Velox on the back

Subject(s): product - film \& paper

Product: Velox paper

Color or Black and White:

Binder: 1923-1925 General Ads \#7

Year: 1924

Slogan: Your X-Ray Darkroom

Subject(s): product - medical

Product: X-Ray darkroom equipment

Color or Black and White:

Binder: 1923-1925 General Ads \#8

Year: Apr-09
Slogan: Take a Kodak to the Fair

Subject(s): Father, son

Text: With a Kodak along you'll make pictures for fun and a

practical record of whatever interests you--cattle, horses, hogs, machinery--pictures full of pointers to apply when you're back on home acres.

Implied Audience: farmers

Product: autographic Kodak

Photo or Illustration Based: photo

Color or Black and White: bw

Description / Notes: Photo of a father and son, from behind,

father with Kodak on his shoulder.

Binder: 1923-1925 General Ads \#8

Year: June / July 1925

Slogan: Let Kodak Save the Day

Subject(s): Two women, baby

Text: Particularly at vacation time, there's so much you want to remember--and pictures won't let you forget. Kodak saves the day--for the years.

Implied Audience: travel

Product: autographic Kodak

Photo or Illustration Based: photo

Color or Black and White: bw

Description / Notes: Photo of a woman with a camera

photographing a woman and baby sitting on the beach underneath an umbrella / parasol.

Binder: 1923-1925 General Ads \#8

Year: Jul-25

Slogan: Kodak as you Go

Subject(s): Two women, two men

Text: The most precious mementos of any trip are the Kodak

pictures you make. Again and again you'll turn to them with everincreasing pleasure. All Kodaks are autographic. You can date and title each exposure as you make it--an exclusive, Eastman feature.

Implied Audience: travelers

Product: autographic Kodak

Photo or Illustration Based: photo 


\section{Color or Black and White: bw}

Description / Notes: Photo of a woman with a camera,

photographing two men and a woman sitting on deck chairs of a ship.

Binder: 1923-1925 General Ads \#8

Year: Jul-25

Slogan: Take a Kodak with You

Subject(s): Woman and man

Text: none

Implied Audience: outdoors

Product: autographic Kodak

Photo or Illustration Based: photo

Color or Black and White: bw

Description / Notes: Photo of a man and woman on a dock, the man helping the woman into a canoe. Woman has a Kodak slung on her back.

Binder: 1923-1925 General Ads \#8

Year: Jul-25

Slogan: Be sure your Brownie goes, too

Subject(s): four boys

Text: Having fun and making pictures travel right along together.

And when the fun is over, you still have the prints. You dad would give a lot for snap-shots of himself and his bunch that were made when he was a boy. Wonder if he knows you'd like a Brownie? It wouldn't do any harm to talk to him about it.

Implied Audience: parents/children

Product: Brownie

Photo or Illustration Based: photo

Color or Black and White: bw

Description / Notes: Photo of four boys, one on the beach, with a camera, photographing the other three in the water, on a log.

Binder: 1923-1925 General Ads \#8

Year: Jul-25

Slogan: Your Brownie Gets It.

Subject(s): Three boys

Text: Your ears are tingling yet from Jack's first attempts at the bugle. But it was a great chance for a picture just the same--and, as usual, your Brownie made the most of it. This Eastman-made camera is certainly easy to work --and there's a barrel full of fun in an album full of pictures.

Implied Audience: parents/children

Product: Brownie

Photo or Illustration Based: photo

Color or Black and White: bw

Description / Notes: Photo of three boys, one photographing

another who is playing the horn, while the third boy points.

Binder: 1923-1925 General Ads \#8

Year: 1925

Slogan: Eastman Low Viscosity Cotton

Subject(s): product - Chemicals

Product: Eastman Organic Chemicals

Color or Black and White:

Binder: 1923-1925 General Ads \#8

Year: 1925

Slogan: Eastman Cotton is Uniform

Subject(s): product - Chemicals

Product: Eastman Organic Chemicals

Color or Black and White:

Binder: 1923-1925 General Ads \#8

Year: 1925

Slogan: An Eastman Organic Chemical

Subject(s): product - Chemicals

Product: Eastman Organic Chemicals

Color or Black and White

Binder: 1923-1925 General Ads \#8

Year: 1925

Slogan: Kodalak Metal Lacquers

Subject(s): product - Chemicals

Product: Eastman Organic Chemicals

Color or Black and White: 
Binder: 1923-1925 General Ads \#8

Year: 1925

Slogan: Announcing the Kodalak Auto Finishing System

Subject(s): product - Chemicals

Product: Eastman Organic Chemicals

Color or Black and White:

Binder: 1923-1925 General Ads \#8

Year: Apr-25

Slogan: Kodalak for Furniture Finishing

Subject(s): product - Chemicals

Product: Eastman Organic Chemicals

Color or Black and White:

Binder: 1923-1925 General Ads \#8

Year: Apr-25

Slogan: Use Eastman Organic Chemicals

Subject(s): product - Chemicals

Product: Eastman Organic Chemicals

Color or Black and White:

Binder: 1923-1925 General Ads \#8

Year: Jun-25

Slogan: The Kodalak System of Automobile Refinishing

Subject(s): product - Chemicals

Product: Eastman Organic Chemicals

Color or Black and White:

Binder: 1923-1925 General Ads \#8

Year: Jun-25

Slogan: Buyers Appreciate Kodalak Finishes

Subject(s): product - Chemicals

Product: Eastman Organic Chemicals

Color or Black and White:

Binder: 1923-1925 General Ads \#8

Year: Jun-25

Slogan: For Acetone Determinations Use an Eastman Organic Chemical
Subject(s): product - Chemicals

Product: Eastman Organic Chemicals

Color or Black and White:

Binder: 1923-1925 General Ads \#8

Year: Jun-25

Slogan: For Aluminum Detection Use Aurin Tricarboxylic Acid an

Eastman Organic Chemical

Subject(s): product - Chemicals

Product: Eastman Organic Chemicals

Color or Black and White:

Binder: 1923-1925 General Ads \#8

Year: 1925

Slogan: The familiar package--dependable results

Subject(s): product - medical

Product: Duplitized X-Ray Films

Description / Notes: Different variations of this ad in the binder.

Binder: 1923-1925 General Ads \#8

Year: May-25

Slogan: Be Sure They Are Eastman Made

Subject(s): product - medical

Product: Duplitized X-Ray Films, Eastman Dental X-Ray films,

Intensifying Screens, Clinical Camera Outfit, Ciné-Kodak

Color or Black and White:

Binder: 1923-1925 General Ads \#8

Year: 1925

Slogan: Eastman Dental X-Ray Films

Subject(s): product - medical

Product: Eastman Dental X-Ray Films

Description / Notes: Different variations of this ad in the

Binder:

Binder: 1923-1925 General Ads \#8

Year: 1925

Slogan: Periodic Radiographs on--Eastman Dental X-Ray Film

Subject(s): product - medical

Product: Eastman Dental X-Ray Films 


\section{Color or Black and White:}

Binder: 1923-1925 General Ads \#8

Year: 1925

Slogan: Kodak Abroad

Subject(s): product - film \& paper

Product: film

Color or Black and White: Publications: L'Atlantique, Ocean

Post, Chicago Tribune, Ocean Times, Daily Mail, Atlantic Edition

Description / Notes: List of European Kodak branches for

development and printing.

Binder: 1923-1925 General Ads \#8

Year: Aug-25

Slogan: Kodak as you go

Subject(s): Family in the car

Text: Round the bend, over the hill, down the lane, there's a

picture at hand or a picture ahead--always.

Implied Audience: travel / family

Product: Kodak

Photo or Illustration Based: photo

Color or Black and White: bw

Description / Notes: Photo of a man, two women, and a boy all in a car.

Binder: 1923-1925 General Ads \#8

Year: 1925

Slogan: No. 1a Pocket Kodak Series II

Subject(s): product - camera

Product: No. 1a Pocket Kodak Series II

Description / Notes: Price: \$26.00 Different variations of this ad

in the

Binder:

Binder: 1923-1925 General Ads \#8

Year: 1925

Slogan: No. 2 Cartridge Hawk-Eye

Subject(s): product - camera

Product: No. 2 Cartridge Hawk-Eye.

Color or Black and White: Publications: Spice Mill

\section{Description / Notes: Sales}

Binder: 1923-1925 General Ads \#8

Year: 1925

Slogan: For Building Business

Subject(s): product - camera

Product: No. 2 Cartridge Hawk-Eye.

Description / Notes: Sales

Binder: 1923-1925 General Ads \#8

Year: 1925

Slogan: The Logical Choice as your premium

Subject(s): product - camera

Product: No. 2 Cartridge Hawk-Eye.

Description / Notes: Sales

Binder: 1923-1925 General Ads \#8

Year: 1925

Slogan: A well-known name for your premium, Premo

Subject(s): product - camera

Product: Premo

Description / Notes: Sales

Binder: 1923-1925 General Ads \#8

Year: 1925

Slogan: Kodak Simplicity further Simplified

Subject(s): product - camera

Product: The 1a Pocket Kodak Series II

Description / Notes: Price: \$26.00 Different variations of this ad

in the

Binder:

Binder: 1923-1925 General Ads \#8

Year: 1925

Slogan: Velox

Subject(s): product - film \& paper

Product: Velox

Color or Black and White:

Binder: 1923-1925 General Ads \#9 
Year: 1925

Slogan: Kodak Keeps the Story

Subject(s): man, woman, child, horse

Text: A Kodak makes it easy to keep the fleeting moments you enjoy. And on the farm with a Kodak you'll make a practical story of your business--a picture record of stock, crops, machinery, that you will refer to with profit from year to year.

Implied Audience: families / farmers

Product: autographic Kodak

Photo or Illustration Based: photo

Color or Black and White: bw

Description / Notes: Photo of a woman with a camera

photographing a young girl on a horse, while a man holds the

horse. Outside a barn.

Binder: 1923-1925 General Ads \#9

Year: 1925

Slogan: Keep the story in Kodak pictures

Subject(s): three men

Text: Thanksgiving Day--volunteer help in the barnyard, hustle and bustle in the kitchen, the rest of the folks expected soon--is a big day for Kodak pictures. You'll find the fun of picture making matched only by the pleasure of picture keeping. And it's all so easy the Kodak way. On your next trip to town stop at your Kodak dealer's and get ready for the pictures you're sure to want.

Implied Audience: families / farmers

Product: Autographic Kodak

Photo or Illustration Based: photo

Color or Black and White: bw

Description / Notes: Photo of three men outside the barn, one

photographing the other two cutting wood with a long saw.

Binder: 1923-1925 General Ads \#9

Year: Nov-25

Slogan: Take a Kodak with you

Subject(s): two men

Text: Gorgeous view or story-telling incident--every trail leads to pictures. Any Kodak is easy to work and convenient to carry, and-as an exclusive Eastman touch--all Kodaks are autographic.
Implied Audience: outdoors

Product: Autographic Kodak

Photo or Illustration Based: photo

Color or Black and White: bw

Description / Notes: Photo of two men (or possibly a man and

woman) sitting on a cliff. One man stands and is taking a photograph.

Binder: 1923-1925 General Ads \#9

Year: 1925

Slogan: Take a Kodak with you

Subject(s): two women, a peddler, one man.

Text: With a Kodak along, your trip is never over--just over the page in your album. Any Kodak is to carry, easy to work; and the exclusively Eastman autographic feature, by means of which the date and title may be written on the film at the time of exposure-for positive and Permanent identification later--is of particular value to the traveler.

Implied Audience: women / travelers

Product: autographic Kodak

Photo or Illustration Based: photo

Color or Black and White: bw

Description / Notes: Photo of a woman with a Kodak photographing another woman buying something from a peddler on the street. A man sits nearby.

Binder: 1923-1925 General Ads \#9

Year: Nov-25

Slogan: Kodak Keeps the Story

Subject(s): two women, man

Text: Almost every day there's sure to be something you'd like a picture of--and with a Kodak you'll find it easy from the first. All Kodaks are autographic--an exclusively Eastman feature.

Whenever you want the date and (or) title--with pictures of the children, for example--you can write it on the film at the time.

Implied Audience: families / farmers

Product: Autographic Kodak

Photo or Illustration Based: photo

Color or Black and White: bw 
Description / Notes: Photo in a cornfield of two women, one with a Kodak photographing a man standing near a stack of corn stalks posing with feathers. The text of the ad has the word 'and' crossed out, and the work 'or' is written above, in ink.

Binder: 1923-1925 General Ads \#9

Year: Dec-25

Slogan: See, I got it, Bill--it's a Brownie

Subject(s): two boys

Text: Brownie cameras (they're Eastman made) cost from $\$ 2$ up.

Implied Audience: parents/children/Christmas

Product: Brownie

Photo or Illustration Based: photo

Color or Black and White: bw

Description / Notes: Photo of two boys on a porch, wreath on

the door behind them. One boy is showing the other his camera.

Binder: 1923-1925 General Ads \#9

Year: 1925

Slogan: Be sure to have your Brownie

Subject(s): two boys and a dog

Text: You'll want it when, after the hike, Davy roasts the "hots"

(and incidentally his finger). You'll want it at the football game, or

out in the woods. A Brownie (Eastman-made) will give you pictures

that will be the envy of the rest of the bunch. Your Kodak dealer

will show you.

Implied Audience: parents/children

Product: Brownie

Photo or Illustration Based: photo

Color or Black and White: bw

Description / Notes: Photo of two boys sitting on a porch, one

handing the Kodak to the other.

Binder: 1923-1925 General Ads \#9

Year: 1925

Slogan: Eastman Low Viscosity Cottons

Subject(s): product - Chemicals

Product: Eastman Organic Chemicals

Color or Black and White:
Binder: 1923-1925 General Ads \#9

Year: 1925

Slogan: The Kodalak System for refinishers

Subject(s): product - Chemicals

Product: Eastman Organic Chemicals

Color or Black and White:

Binder: 1923-1925 General Ads \#9

Year: 1925

Slogan: Five new Eastman Organic Chemicals are now in stock

Subject(s): product - Chemicals

Product: Eastman Organic Chemicals

Color or Black and White:

Binder: 1923-1925 General Ads \#9

Year: 1925

Slogan: Save valuable time by using Eastman Organic Chemicals

Subject(s): product - Chemicals

Product: Eastman Organic Chemicals

Color or Black and White:

Binder: 1923-1925 General Ads \#9

Year: 1925

Slogan: It is cheaper to use Eastman Organic Chemicals

Subject(s): product - Chemicals

Product: Eastman Organic Chemicals

Color or Black and White:

Binder: 1923-1925 General Ads \#9

Year: 1925

Slogan: Eastman Cotton

Subject(s): product - Chemicals

Product: Eastman Organic Chemicals

Color or Black and White:

Binder: 1923-1925 General Ads \#9

Year: 1925

Slogan: Kodalak Finishes for Lighting Fixtures 
Subject(s): product - Chemicals

Product: Eastman Organic Chemicals

Color or Black and White:

Binder: 1923-1925 General Ads \#9

Year: 1925

Slogan: Retailers like Kodalak finishes

Subject(s): product - Chemicals

Product: Eastman Organic Chemicals

Color or Black and White:

Binder: 1923-1925 General Ads \#9

Year: 1925

Slogan: Save by using Kodalak Furniture Finishes

Subject(s): product - Chemicals

Product: Eastman Organic Chemicals

Color or Black and White:

Binder: 1923-1925 General Ads \#9

Year: 1925

Slogan: Eastman Organic Chemicals

Subject(s): product - Chemicals

Product: Eastman Organic Chemicals

Description / Notes: Different variations of this ad in this Binder:

Binder: 1923-1925 General Ads \#9

Year: 1925

Slogan: The Kodalak System is fast and reliable

Subject(s): product - Chemicals

Product: Eastman Organic Chemicals

Color or Black and White:

Binder: 1923-1925 General Ads \#9

Year: 1925

Slogan: Eastman Organic Chemical

Subject(s): product - Chemicals

Product: Eastman Organic Chemicals

Description / Notes: Different variations of this ad in this Binder:
Binder: 1923-1925 General Ads \#9

Year: 1925

Slogan: Eastman Cotton for pyroxylin lacquers

Subject(s): product - Chemicals

Product: Eastman Organic Chemicals

Color or Black and White:

Binder: 1923-1925 General Ads \#9

Year: 1925

Slogan: Eastman Low Viscosity Cotton

Subject(s): product - Chemicals

Product: Eastman Organic Chemicals

Color or Black and White:

Binder: 1923-1925 General Ads \#9

Year: 1925

Slogan: A High Luster with Kodalak

Subject(s): product - Chemicals

Product: Eastman Organic Chemicals

Color or Black and White:

Binder: 1923-1925 General Ads \#9

Year: 1925

Slogan: Over 2,000 Eastman Organic Chemicals

Subject(s): product - Chemicals

Product: Eastman Organic Chemicals

Color or Black and White:

Binder: 1923-1925 General Ads \#9

Year: 1925

Slogan: Kodalak Furniture Finishes

Subject(s): product - Chemicals

Product: Eastman Organic Chemicals

Color or Black and White:

Binder: 1923-1925 General Ads \#9

Year: 1925

Slogan: Eastman Carbon Tetrachloride, Sulfur Free Subject(s): product - Chemicals 
Product: Eastman Organic Chemicals Color or Black and White:

Binder: 1923-1925 General Ads \#9

Year: 1925

Slogan: For Radiographs of Gall Bladder Tetraiodophenolphthalein Sodium Salt

Subject(s): product - Chemicals

Product: Eastman Organic Chemicals

Color or Black and White:

Binder: 1923-1925 General Ads \#9

Year: 1925

Slogan: Two Reasons for the Determination of Copper

Subject(s): product - Chemicals

Product: Eastman Organic Chemicals

Color or Black and White:

Binder: 1923-1925 General Ads \#9

Year: 1925

Slogan: A Kodalak Finish Helps

Subject(s): product - Chemicals

Product: Eastman Organic Chemicals

Color or Black and White:

Binder: 1923-1925 General Ads \#9

Year: 1925

Slogan: Measuring the Acidity of Plant Juices

Subject(s): product - Chemicals

Product: Eastman Organic Chemicals

Color or Black and White:

Binder: 1923-1925 General Ads \#9

Year: 1925

Slogan: Appearance Sells Furniture

Subject(s): product - Chemicals

Product: Eastman Organic Chemicals

Color or Black and White:
Binder: 1923-1925 General Ads \#9

Year: 1925

Slogan: Kodaloid

Subject(s): product - Chemicals

Product: Eastman Organic Chemicals

Color or Black and White:

Binder: 1923-1925 General Ads \#9

Year: 1925

Slogan: Eastman Chemical Charcoal

Subject(s): product - Chemicals

Product: Eastman Organic Chemicals

Color or Black and White

Binder: 1923-1925 General Ads \#9

Year: 1925

Slogan: Eastman Methanol, pure

Subject(s): product - Chemicals

Product: Eastman Organic Chemicals

Color or Black and White:

Binder: 1923-1925 General Ads \#9

Year: 1925

Slogan: Eastman Methyl Acetone

Subject(s): product - Chemicals

Product: Eastman Organic Chemicals

Color or Black and White:

Binder: 1923-1925 General Ads \#9

Year: 1925

Slogan: Kodalak Furniture Finishes

Subject(s): product - Chemicals

Product: Eastman Organic Chemicals

Color or Black and White:

Binder: 1923-1925 General Ads \#9

Year: 1925

Slogan: Use Kodaloid

Subject(s): product - Chemicals 
Product: Eastman Organic Chemicals Color or Black and White:

Binder: 1923-1925 General Ads \#9 Year: 1925

Slogan: Eastman Films must be Noticably Better

Subject(s): product - medical

Product: Duplitized X-Ray Films

Color or Black and White:

Binder: 1923-1925 General Ads \#9

Year: 1925

Slogan: Story Telling Radiographs

Subject(s): product - medical

Product: Duplitized X-Ray Films

Color or Black and White:

Binder: 1923-1925 General Ads \#9

Year: 1925

Slogan: Eastman Dupli-Tized Safety X-Ray Films

Subject(s): product - medical

Product: Duplitized X-Ray Films

Color or Black and White:

Binder: 1923-1925 General Ads \#9

Year: 1925

Slogan: Eastman Safety Dupli-Tized X-Ray Films Super-Speed

Subject(s): product - medical

Product: Duplitized X-Ray Films

Description / Notes: Different variations of this ad in the binder.

Binder: 1923-1925 General Ads \#9

Year: 1925

Slogan: To be sure use Eastman Dupli-Tized X-Ray Film

Subject(s): product - medical

Product: Duplitized X-Ray Films

Color or Black and White:

Binder: 1923-1925 General Ads \#9
Year: 1925

Slogan: Is an Operation Indicated? Eastman Dupli-Tized X-Ray

Films Super-Speed

Subject(s): product - medical

Product: Duplitized X-Ray Films

Color or Black and White:

Binder: 1923-1925 General Ads \#9

Year: 1925

Slogan: Eastman Safety Films Reduce the Fire Hazard

Subject(s): product - medical

Product: Duplitized X-Ray Films

Color or Black and White:

Binder: 1923-1925 General Ads \#9

Year: 1925

Slogan: Radiographs

Subject(s): product - medical

Product: Duplitized X-Ray Films

Description / Notes: Different variations of this ad in the

Binder:

Binder: 1923-1925 General Ads \#9

Year: 1925

Slogan: Eastman Aero Camera, K-5

Subject(s): product - camera

Product: Eastman Aero Camera, K-5

Color or Black and White

Binder: 1923-1925 General Ads \#9

Year: 1925

Slogan: Orthodontia and Photography

Subject(s): product - medical

Product: Eastman Clinical Camera

Color or Black and White:

Binder: 1923-1925 General Ads \#9

Year: 1925

Slogan: Use Radiographs to locate trouble Below the Gum Line Subject(s): product - medical 
Product: Eastman Dental X-Ray Films

Color or Black and White:

Binder: 1923-1925 General Ads \#9

Year: 1925

Slogan: The use of Radiographs Marks Progress of Dentistry

Subject(s): product - medical

Product: Eastman Dental X-Ray Films

Color or Black and White:

Binder: 1923-1925 General Ads \#9

Year: 1925

Slogan: Dental Abnormalities

Subject(s): product - medical

Product: Eastman Dental X-Ray Films

Color or Black and White:

Binder: 1923-1925 General Ads \#9

Year: 1925

Slogan: Pulpless Teeth

Subject(s): product - medical

Product: Eastman Dental X-Ray Films

Color or Black and White:

Binder: 1923-1925 General Ads \#9

Year: 1925

Slogan: For your Fall campaign an Eastman Camera

Subject(s): product - camera

Product: Hawk-eye and Premo

Description / Notes: Sales

Binder: 1923-1925 General Ads \#9

Year: Dec-25

Slogan: The best surprise of all--a Kodak

Subject(s): Mother, father, young boy

Text: none

Implied Audience: families / Christmas

Product: Kodak

Photo or Illustration Based: photo
Color or Black and White: bw

Description / Notes: Photo of a mother walking down the stairs, a father and young boy waiting by the Christmas tree, father is

holding a Kodak behind his back.

Binder: 1923-1925 General Ads \#9

Year: 1925

Slogan: All Outdoors invites your Kodak

Subject(s): Woman and dog

Text: Any Kodak is easy to work, as you'll see for yourself at your dealer's.

Implied Audience: women / outdoors

Product: Kodak

Photo or Illustration Based: photo

Color or Black and White: bw

Description / Notes: Photo of a woman in fur, holding a Kodak, standing on a hill with a dog.

Binder: 1923-1925 General Ads \#9

Year: Dec-25

Slogan: A Christmas Kodak for the Christmas Story

Subject(s): Women, two men

Text: none

Implied Audience: families / Christmas

Product: Kodak

Photo or Illustration Based: photo

Color or Black and White: bw

Description / Notes: Photo of a woman with a Kodak standing at

a window, beside a Christmas tree. She is looking outside at two

men in a car, waving at her.

Binder: 1923-1925 General Ads \#9

Year: 1925

Slogan: No. 1a Pocket Kodak Series II

Subject(s): product - camera

Product: No. 1a Pocket Kodak Series II

Description / Notes: Price: $\$ 26.00$ - different variations in this binder with different example images. 
Binder: 1923-1925 General Ads \#9

Year: 1925

Slogan: No. 1a Pocket Kodak Series II

Subject(s): product - camera

Product: No. 1a Pocket Kodak Series II

Description / Notes: Price: \$26 Pictures 2.5 x 4.25

Binder: 1923-1925 General Ads \#9

Year: 1925

Slogan: Something that Everybody Wants, Hawk-Eye Cameras

Subject(s): product - camera

Product: No. 2 Cartridge Hawk-Eye, No. 2 Folding Cartridge

Hawk-Eye

Description / Notes: Sales

Binder: 1923-1925 General Ads \#9

Year: 1925

Slogan: Boost Fall business with a camera

Subject(s): product - camera

Product: No. 2 Cartridge Hawk-Eye.

Description / Notes: Sales

Binder: 1923-1925 General Ads \#9

Year: 1925

Slogan: As a Prize or Premium--No. 2 Cartridge Hawk-Eye

Subject(s): product - camera

Product: No. 2 Cartridge Hawk-Eye.

Description / Notes: Sales

Binder: 1923-1925 General Ads \#9

Year: 1925

Slogan: The Logical Premium--No. 2 Cartridge Hawk-Eye

Subject(s): product - camera

Product: No. 2 Cartridge Hawk-Eye.

Description / Notes: Sales

Binder: 1923-1925 General Ads \#9

Year: 1925

Slogan: All Season Business Builders
Subject(s): product - camera

Product: No. 2 Cartridge Hawk-Eye.

Description / Notes: Sales

Binder: 1923-1925 General Ads \#9

Year: 1925

Slogan: Vest Pocket Kodak

Subject(s): product - camera

Product: Vest Pocket Kodak Model B

Description / Notes: Price: $\$ 5.00$ 